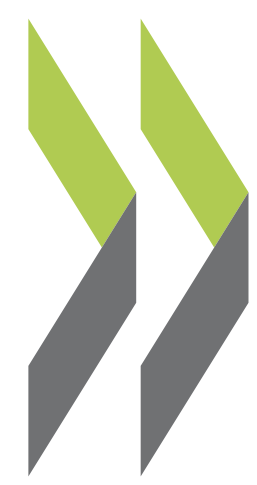

Documents SIGMA No. 54

Développer des relations de travail efficaces entre les institutions supérieures de contrôle et les parlements
Bianca Brétéché,

\section{Alastair Swarbrick}

https://dx.doi.org/10.1787/742ee4b5-fr 


\title{
Développer des relations de travail efficaces entre les institutions supérieures de contrôle et les parlements
}

\author{
DOCUMENT SIGMA N .54
}


TABLE DES MATIÈRES

SYNTHÈSE . .4

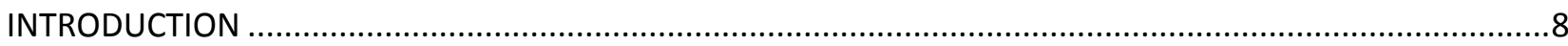

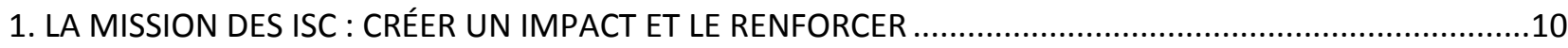

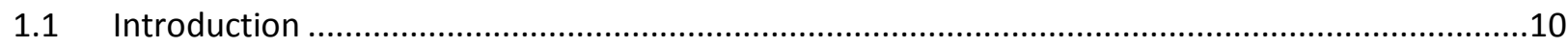

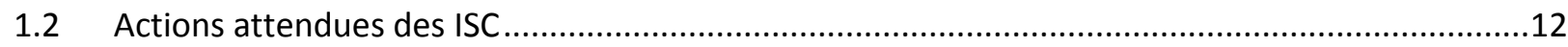

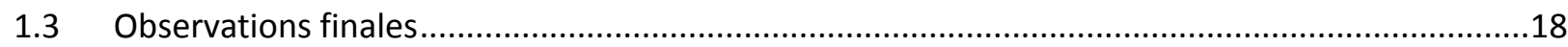

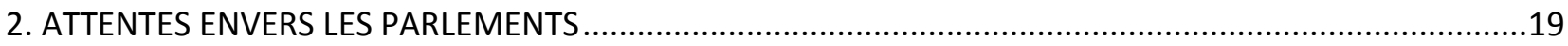

2.1 Mécanismes officiels dédiés au traitement des rapports des ISC ............................................20

2.2 Attentes envers les commissions d'audit parlementaires (PAC) .............................................21

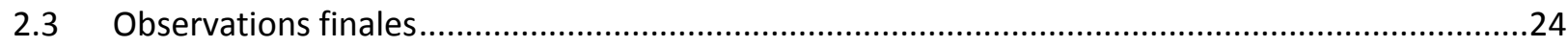

3. RELATIONS ENTRE LES ISC ET LE PARLEMENT : QUELQUES FACTEURS CONTEXTUELS..........................25

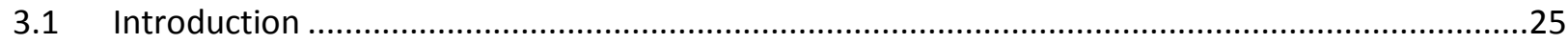

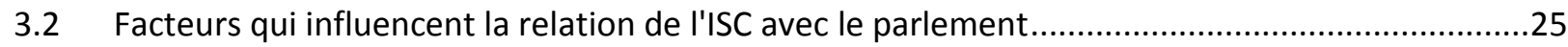



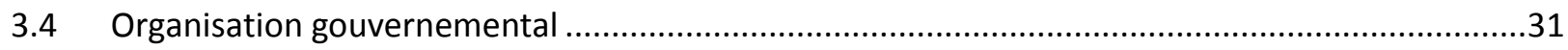

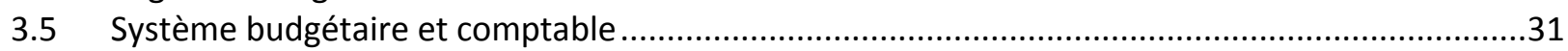

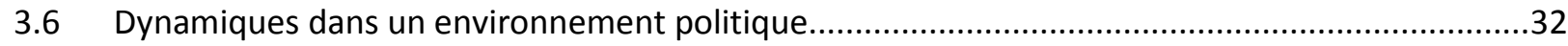



4. ANALYSE DES RELATIONS DE TRAVAIL ENTRE LES ISC ET LES PARLEMENTS : BONNES PRATIQUES EN

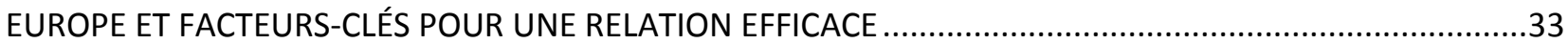

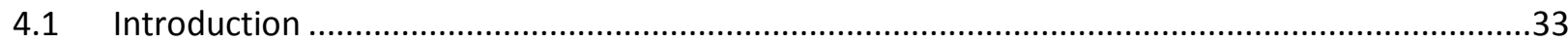

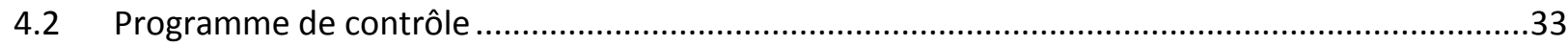

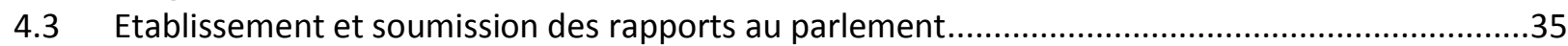

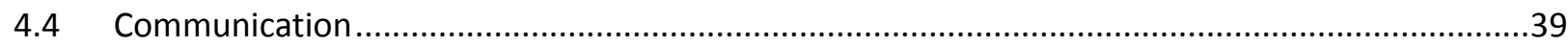

4.5 Suivi des observations précédentes et des recommandations de l'ISC .......................................40

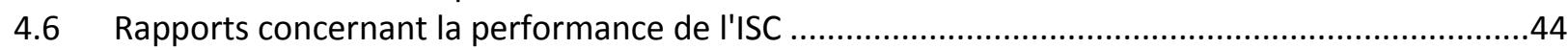

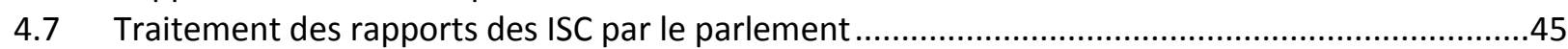

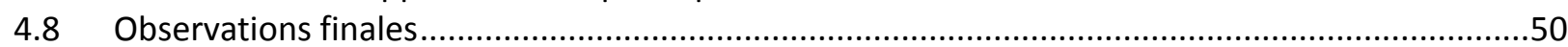

ANNEXE 1. NORMES INTERNATIONALES ET PRINCIPES RECONNUS PORTANT SUR LES RELATIONS ENTRE

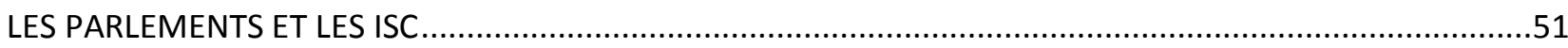

ANNEXE 2. BOÎTE À OUTILS DESTINÉE À RENFORCER LES RELATIONS DE TRAVAIL ENTRE LES ISC ET LE

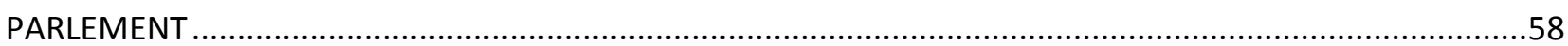

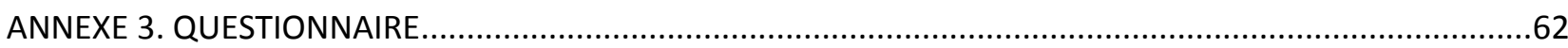

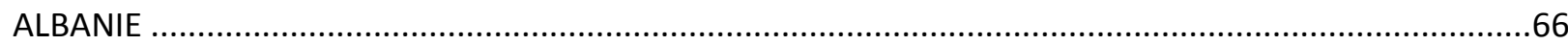

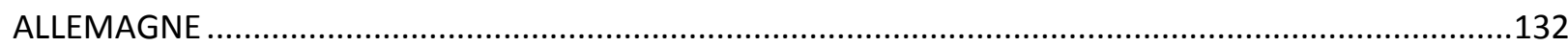






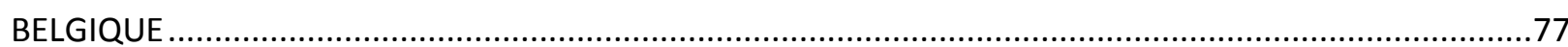



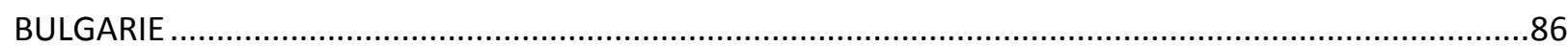

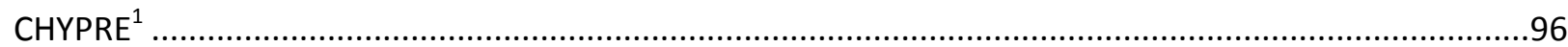

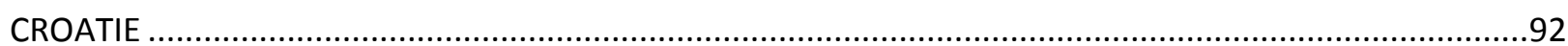

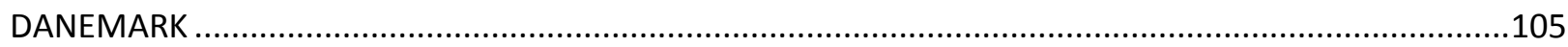

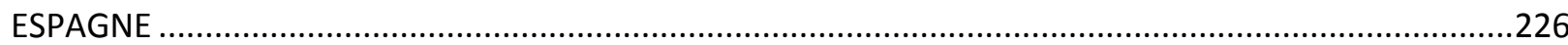





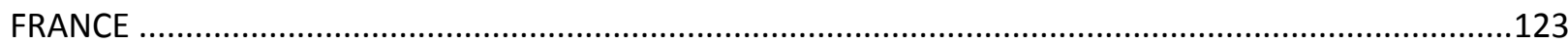

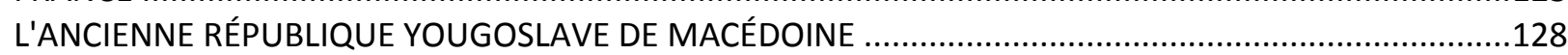

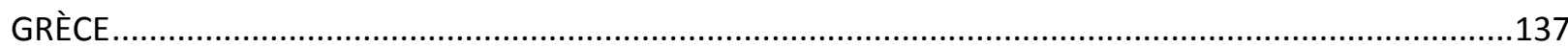

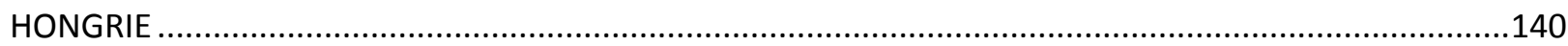



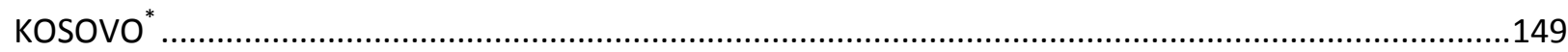



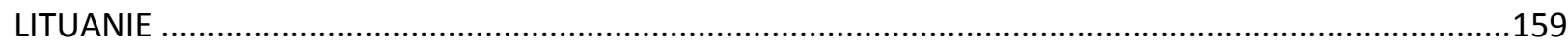

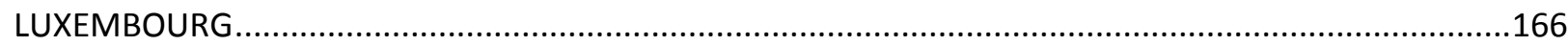

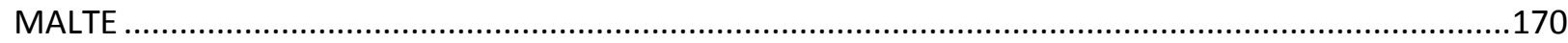

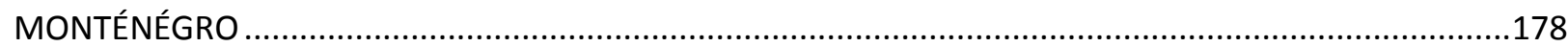

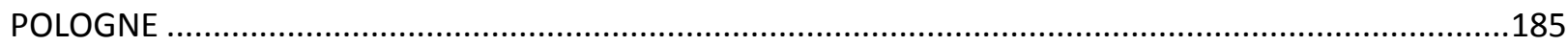

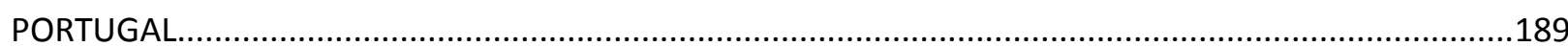



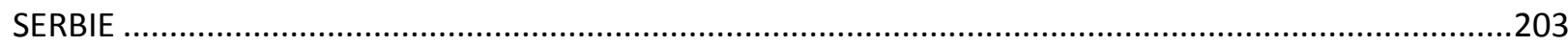

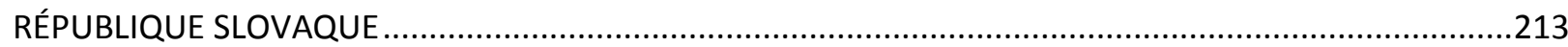



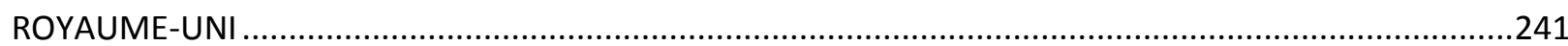

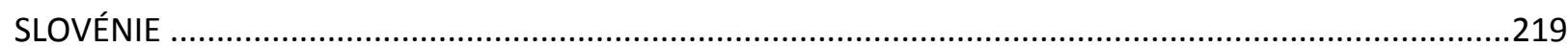

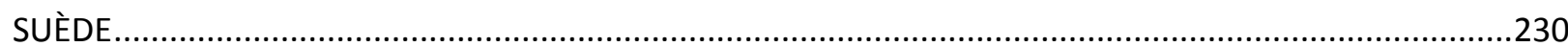

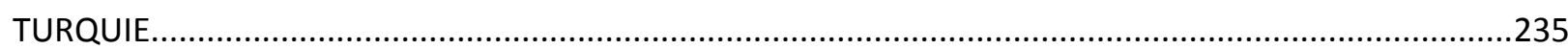

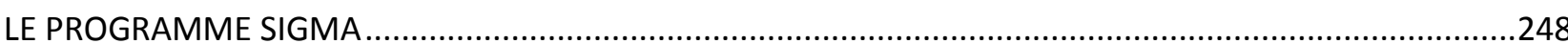

1. $\quad$ Note de la Turquie

L'information transmise dans le présent document et qui fait référence à " Chypre » concerne la partie sud de l'île. II n'y a pas d'autorité unique représentant à la fois la population turque et la population grecque chypriote de l'île. La Turquie reconnaît la République turque du nord de Chypre (TRNC). Jusqu'à ce qu'une solution durable et équitable soit trouvée au sein des Nations-Unies, la Turquie restera sur sa position au regard de la « question chypriote ».

Note de tous les États membres de l'Union européenne, de l'OCDE et de l'Union européenne

La République de Chypre est reconnue par tous les membres des Nations-Unies à l'exception de la Turquie. L'information transmise dans le présent document concerne la région sous le contrôle effectif du gouvernement de la République de Chypre.

* $\quad$ Cette désignation ne préjuge pas des positions sur le statut, et elle respecte la Résolution du Conseil de Sécurité des Nations-Unies 1244/99 et de l'Opinion consultative de la Cour internationale de justice sur la déclaration d'indépendance du Kosovo. 


\section{SYNTHÈSE}

Les institutions supérieures de contrôle (ISC) et les parlements sont les deux plus importants protagonistes habilités à demander aux gouvernements de rendre compte de leur utilisation des finances publiques. Généralement, les parlements n'ont pas les moyens ou l'expertise pour examiner la manière dont les gouvernements utilisent les fonds publics. Ils s'appuient sur les travaux objectifs et professionnels des ISC qui leur fournissent une garantie et des informations concernant la fiabilité des rapports financiers et l'utilisation des ressources publiques.

Les parlements auront toutefois recours aux travaux des ISC uniquement s'ils sont pertinents et compréhensibles dans un contexte politique donné. C'est pourquoi il est important que les ISC, dans le cadre de leur mission de contrôle de la gestion publique, veillent à ce que leurs travaux soient pertinents, qu'ils ajoutent de la valeur et qu'ils aient un impact, pas uniquement par le biais d'analyses et de rapports sur les faits passés, mais également en se tournant vers l'avenir, en identifiant les points sur lesquels des améliorations sont possibles, et en encourageant les bonnes pratiques. De cette manière, l'audit du secteur public peut contribuer à l'amélioration des normes de gouvernance, du management et du processus de décision, ainsi qu'à une utilisation plus efficace des deniers publics.

La présente étude propose aux ISC et aux parlements des orientations visant à établir des relations de travail efficaces. Elle présente et analyse les normes internationales et les facteurs contextuels, ainsi que les spécificités et les pratiques existant dans l'ensemble de l'Europe. Par ailleurs, elle met en lumière les éléments essentiels à la mise en place de relations efficaces et de bonnes pratiques. Elle offre également une boîte à outils qui permet de renforcer les relations de travail entre les ISC et les parlements. Ce rapport est le fruit de la contribution de trente-trois ISC de pays membres de I'Union européenne (EU) et de pays candidats et candidats potentiels.

La présente étude décrit les normes internationales actuelles, qui comportent de fortes attentes concernant la manière dont les ISC rendent compte de leurs travaux d'audit aux parlements, les efforts qu'elles doivent entreprendre pour aider les parlementaires à comprendre et à utiliser les rapports d'audit. Les ISC doivent en outre avoir des échanges réguliers avec leur parlement afin de connaître leurs attentes, et de s'assurer que les parlementaires comprennent le rôle de I'ISC et le bénéfice qu'ils peuvent retirer de son travail.

Le présent document aborde par ailleurs un certain nombre de facteurs qui influencent ces relations, facteurs à prendre en compte quand il s'agit de mettre en œuvre des dispositions pratiques pour le dialogue entre I'ISC et le parlement. Le contexte constitutionnel et historique de I'ISC, le système parlementaire, l'organisation gouvernementale et le système budgétaire et comptable, tous ces éléments jouent un rôle dans les relations entre l'ISC et le parlement.

En réponse à l'enquête réalisée par SIGMA au début de l'année 2016, trente-trois ISC ont décrit comment elles répondaient à ces attentes. En outre, elles ont fourni des informations sur la façon dont leur parlement utilise leur travail. Les résultats de l'enquête ont confirmé que toutes les ISC examinaient en permanence la manière dont elles pouvaient étendre leur influence ; pour cela, elles élaborent de nouvelles procédures, elles ciblent leur communication, elles imposent davantage de transparence et elles adoptent une attitude proactive. La diversité et la variété des pratiques communiquées est une source d'inspiration pour toutes les ISC qui ont à cœur de renforcer l'impact de leurs relations de travail avec le parlement. Dans une certaine mesure, ceci vaut également pour les parlements qui considèrent de 
leur devoir d'utiliser les rapports de I'ISC dans le but de mettre le gouvernement devant ses responsabilités.

Des relations de travail efficaces commencent par différents moyens de coopération dès la planification par les ISC de leurs programmes de travail annuels et pluriannuels. La majorité des ISC tiennent compte des suggestions spécifiques émises par leur parlement, qu'il existe ou non une disposition juridique permettant au parlement de solliciter des audits. Parmi les bonnes pratiques relevées dans ce sens, il y a l'instauration, en concertation avec le parlement, d'une procédure de consultation encadrant les demandes d'audits et la limitation du nombre d'audits à exécuter sur demande. Ces bonnes pratiques consistent également à informer le parlement du programme de travail de I'ISC et de son plan de contrôle stratégique, à offrir au parlement la possibilité d'apporter régulièrement sa contribution, à être en liaison avec la commission compétente et les autres commissions concernées, et à suivre les débats du parlement dans le but d'identifier des audits potentiels.

Les rapports d'audit que les ISC envoient au parlement constituent le socle de leur relation mutuelle. Tandis que toutes les ISC européennes rendent compte au parlement, il existe des différences substantielles entre les pays quant aux moyens employés, à la période et à la fréquence des échanges. Les bonnes pratiques adoptées par les ISC dans leur communication avec le parlement incluent les tâches suivantes :

- Soumettre les rapports au parlement et les publier simultanément ;

- S'assurer que les rapports sont remis à l'ensemble des commissions / membres concernés ;

- Proposer des présentations et des notes d'information sur les rapports ;

- Diffuser des communiqués de presse pour mettre en lumière les questions importantes traitées dans les rapports ;

- Sélectionner les rapports à soumettre au parlement ou donner un avis consultatif sur les rapports à sélectionner pour examen ;

- Envisager d'établir des rapports thématiques, qui regroupent les résultats d'audits précédents.

De nombreuses ISC ne se contentent pas de remettre au parlement des rapports d'audit. Elles prennent l'initiative d'établir de bonnes relations de travail avec leur parlement, de le sensibiliser à leur mission et de l'aider à comprendre les rapports d'audit. Ces actions de communication visent par ailleurs à renforcer l'attention que les parlements accordent aux rapports des ISC. Les bonnes pratiques portant sur la communication avec les parlements comprennent les tâches suivantes : tenir régulièrement des réunions, complétées par des échanges informels et des entretiens au niveau opérationnel, coordonner les agendas et les calendriers de soumission des rapports, organiser des conférences, des tables rondes et des ateliers, signer des protocoles d'accord sur les procédures de coopération, élaborer une politique de communication et améliorer les connaissances réciproques en proposant le détachement de personnel.

Pour coopérer avec les ISC et utiliser leurs travaux pour obliger les gouvernements à rendre compte, la méthode adoptée par les parlements consiste le plus souvent à déléguer cette tâche à une commission. Dans tous les parlements européens, cette tâche est attribuée aux commissions budgétaires ou à des commissions de contrôle spécifiques aux instances publiques, qui peuvent être des commissions ou des sous-commissions permanentes. Dans certains pays, les commissions sectorielles interviennent également dans I'incitation à utiliser les travaux d'audit des ISC. Les bonnes pratiques identifiées dans le cadre des méthodes parlementaires consistent notamment à : 
- Fixer de manière adéquate les responsabilités de la commission chargée d'examiner les rapports de l'ISC ;

- Mettre en place une commission ou sous-commission de contrôle spécialisée ;

- Mettre à la disposition du parlement un personnel ainsi que des capacités d'analyse suffisantes ;

- Solliciter les commissions sectorielles, notamment dans le traitement des rapports d'audit de performance ;

- Intégrer dans le cycle budgétaire une procédure de décharge formalisée.

Pour que leurs travaux d'audit donnent des résultats et contribuent à améliorer la gestion des fonds publics, toutes les ISC européennes assurent le suivi de leurs observations et de la mise en œuvre des recommandations figurant dans leurs rapports d'audit. De nombreuses ISC gèrent en outre une base de données, et certaines publient des informations sur les mesures prises par les institutions publiques en réponse aux constatations des audits. D'autres bonnes pratiques identifiées dans le cadre de procédures de suivi comprennent l'établissement de rapports sur la mise en œuvre des recommandations à intervalles fixes, l'intégration du système de suivi dans celui de la planification des audits de vérification et de suivi, et l'intérêt particulier accordé à la communication portant sur la mise en œuvre des recommandations figurant dans les rapports d'audit de performance.

Les travaux d'audit réalisés par les ISC ont davantage d'impact si les parlements assurent eux-aussi le suivi des travaux d'audit en exerçant leur fonction de surveillance budgétaire envers le gouvernement. De nombreux parlements européens ont mis en place des procédures adéquates. Les bonnes pratiques incluent les exemples suivants :

- Mise en place de procédures et de calendriers normalisés régissant l'examen parlementaire des rapports de I'ISC, pour pouvoir formuler des conclusions en temps opportun ;

- Désignation d'un rapporteur pour certains rapports de l'ISC ;

- Organisation d'auditions en présence des entités contrôlées ;

- Demander au gouvernement ou à l'entité contrôlée d'établir des plans d'action, et assortir les mesures à prendre d'un calendrier d'exécution ;

- Envisager des sanctions en cas de non-conformité grave au regard des recommandations formulées par l'ISC ou par le parlement (sanction politique, financière et disciplinaire) ;

- Exiger de l'entité contrôlée ou du gouvernement des rapports sur la mise en œuvre des actions appropriées.

Pour témoigner de leur responsabilité dans l'exercice de leur travail, presque toutes les ISC rendent compte au parlement de leur performance. Concrètement, elles dressent des rapports sur leurs activités et leur utilisation des ressources pendant l'année antérieure, soit dans un rapport d'activité annuel séparé, soit dans un chapitre spécial du rapport annuel. À cet égard, les bonnes pratiques impliquent de remettre au parlement un rapport annuel certifié sur les comptes et l'utilisation des ressources et un rapport annuel sur les activités et la réalisation du programme de travail. On peut également citer les examens réalisés régulièrement par les pairs, et l'estimation des économies obtenues dans le secteur public.

En lien avec les normes et principes applicables, ces bonnes pratiques constituent une " boîte à outils destinée à renforcer les relations entre les ISC et les parlements " figurant à l'Annexe 2. II n'y a pas de 
modèle unique " prêt à porter " pour instaurer des relations efficaces entre les ISC et les parlements, et au fil du temps, ces relations nécessitent d'être remaniées. Cette boîte à outils peut cependant contribuer à identifier de nouveaux instruments ou de nouvelles procédures dans le but de maintenir et d'améliorer des relations de travail efficaces. 


\section{INTRODUCTION}

Depuis quelques années, la communauté des ISC étudie l'influence de leurs travaux et dans quelle mesure ces derniers peuvent contribuer à changer les choses dans la vie des citoyens. L'approbation en 2013 de la Norme Internationale des Institutions supérieures de contrôle ${ }^{1}$ (ISSAI) 12 La Valeur et les avantages des $I S C^{2}$ élaborée par l'Organisation internationale des Institutions supérieures de contrôle (INTOSAI) montre sans ambigüité le rôle important joué par les ISC pour améliorer l'utilisation des ressources publiques et encourager davantage d'efficacité des services publics. La mise en place d'une relation efficace entre les ISC et leur parlement respectif est une composante essentielle de cette ambition.

Les ISC appartenant au réseau des pays candidats et candidats potentiels de I'UE (le Réseau) ont déclaré que "le développement de l'influence des ISC » était une priorité commune lors de leur réunion d'Istanbul en 2011, notamment en coopérant avec les parlements nationaux. En novembre 2013, I'ISC du Monténégro a accueilli une conférence du Réseau ayant pour sujet les relations entre les ISC et les parlements ; lors de cette conférence, il a été décidé de rédiger le présent document d'orientation, son but étant de fournir des exemples de bonnes pratiques dans la création et la gestion de relations efficaces avec le parlement, mais également de contribuer à définir les opportunités de consolider une relation de travail mutuellement bénéfique. SIGMA a accepté d'aider le Réseau à élaborer le présent document d'orientation.

Le principal objectif du présent document d'orientation est de présenter une vue d'ensemble des pratiques utilisées au sein de I'UE et des pays du Réseau afin de fortifier les relations entre les ISC et les parlements. Il définit les facteurs cruciaux à la base d'une relation efficace et il cerne les bonnes pratiques susceptibles d'être appliquées. II examine les attentes concernant ces relations, établies par différentes normes, documents d'orientation et études, ainsi que l'impact des structures et des traditions politiques, constitutionnelles, juridiques et administratives sur les organes institutionnels et sur les systèmes de responsabilisation des gestionnaires publics. L'objectif global vise à offrir au Réseau des idées et des solutions destinées à améliorer la coopération nouée par ses membres avec leur parlement respectif, tout en continuant à renforcer leurs capacités d'accroître l'impact de leurs audits.

Le présent document a été élaboré en tenant compte des exigences des ISSAI et autres normes, de la documentation à disposition du public et des contributions obtenues par l'enquête conduite auprès des ISC des Pays membres de I'UE et du Réseau. Sur les trente-six institutions invitées à participer, trentetrois ont répondu, et les informations tirées de cette enquête constituent la Partie 2 du présent document.

Le chapitre 1 définit le contexte théorique de l'obligation de rendre compte ; il examine les normes et les principes pertinents dont l'observance est nécessaire pour que les ISC travaillent efficacement avec les parlements. Le chapitre 2 aborde les attentes existantes de la part des parlements. Le chapitre 3

$1 \quad$ Normes Internationales des Institutions supérieures de contrôle (ISSAI), http://www.issai.org/.

2 ISSAI 12, La valeur et les avantages des institutions supérieures de contrôle des finances publiques - faire une différence dans la vie des citoyens (adoptée en 2013), disponible sur http://www.issai.org/en us/siteissai/issai-framework/2-prerequisites-for-the-functioning-of-sais.htm. 
analyse le contexte dans lequel s'inscrivent les relations entre les ISC et les parlements. Le chapitre 4 examine les relations actuelles dans le but d'instaurer les bonnes pratiques correspondant aux normes et principes définis aux chapitres 1 et 2 . II en résulte ce que l'on pourrait appeler une " boîte à outils " de bonnes pratiques dont les ISC et les parlements peuvent profiter lorsqu'ils souhaitent renforcer l'efficacité de leurs relations.

Le présent document, portant sur les relations des ISC avec leur parlement, se concentre sur la manière dont une ISC peut accroître l'impact de ses travaux. En conséquence, l'étude s'est limitée à examiner les domaines directement concernés par une utilisation efficace et par l'impact des rapports d'audit et d'autres travaux exécutés par les ISC, comme les avis et les conseils. Les autres domaines où les intérêts de I'ISC et du parlement se croisent, par exemple la procédure parlementaire de désignation de l'auditeur général ou du président de I'ISC, ou bien l'adoption du budget de I'ISC, n'ont pas été pris en compte.

Les contributions des ISC des Pays membres de l'UE et du Réseau, et tout particulièrement le soutien du groupe de travail des ISC de la Turquie, de l'Albanie, du Kosovo et de la Serbie, à l'élaboration du présent document, ont été vivement appréciées. Ce rapport a été rédigé avec les contributions expertes de Messieurs Jan Pieter Lingen et Klaus Goetz, et revu par M. Alastair Swarbrick et Mme Bianca Brétéché. 


\section{LA MISSION DES ISC : CRÉER UN IMPACT ET LE RENFORCER}

\subsection{Introduction}

Selon la Déclaration de Lima (ISSAI 1, section 1) ${ }^{3}$ :

"L'institution du contrôle est immanente à l'administration des finances publiques laquelle constitue une gestion fiduciaire. Le contrôle des finances publiques n'est pas une fin en soi mais il est un élément indispensable d'un système régulatoire qui a pour but de signaler en temps utile les écarts par rapport à la norme ou les atteintes aux principes de la conformité aux lois, de l'efficience, de l'efficacité et de l'économie de la gestion financière de manière à ce que l'on puisse, dans chaque cas, prendre des mesures correctives, préciser la responsabilité des parties en cause, obtenir réparation ou prendre des mesures pour empêcher, ou du moins rendre plus difficile, la perpétration d'actes de cette nature. »

Dans un système rigoureux de gestion et de contrôle des finances publiques, les responsables des affaires publiques et utilisant les ressources publiques seront tenus de rendre compte de leur administration à ceux qui utilisent et payent les services fournis, conformément à la loi et aux normes en vigueur. II convient de préserver les ressources publiques, de justifier de leur emploi, et de les utiliser de manière économique, efficiente et efficace.

Un des rôles fondamentaux d'un parlement est d'autoriser le budget de l'État et de tenir le gouvernement responsable de son exécution, en s'assurant, pour le compte des citoyens et des utilisateurs des services publics, que les ressources sont utilisées de manière régulière et responsable, pour les objectifs prévus, et avec un souci d'économie, d'efficience et d'efficacité. Pour remplir efficacement cette mission, les membres du parlement ont besoin d'informations objectives et factuelles sur la façon dont le gouvernement collecte et dépense les fonds publics.

Les ISC jouent un rôle important dans ce système de responsabilisation entre le parlement et le gouvernement (l'exécutif), et à l'extérieur envers les citoyens et les utilisateurs des services publics. Elles fournissent des rapports et des informations indépendantes et objectives sur : la fiabilité des rapports financiers du gouvernement, son utilisation des ressources, la préservation des actifs et des ressources qui lui sont confiés, et le respect des lois, des règlements et des autres autorités pertinentes qui habilitent les parlements (et aux autres parties prenantes) à demander des comptes au gouvernement. La raison fondamentale de l'existence des ISC est de fournir une garantie et une information crédible aux parties prenantes, dans l'intérêt du public.

Le lien entre les ISC, leur parlement et leur gouvernement est illustré avec clarté par le triangle des responsabilités (voir figure 1). Au sein de cette structure, les ISC doivent réfléchir sur le moyen d'exercer au mieux leur fonction en lien avec les autres acteurs du système. 


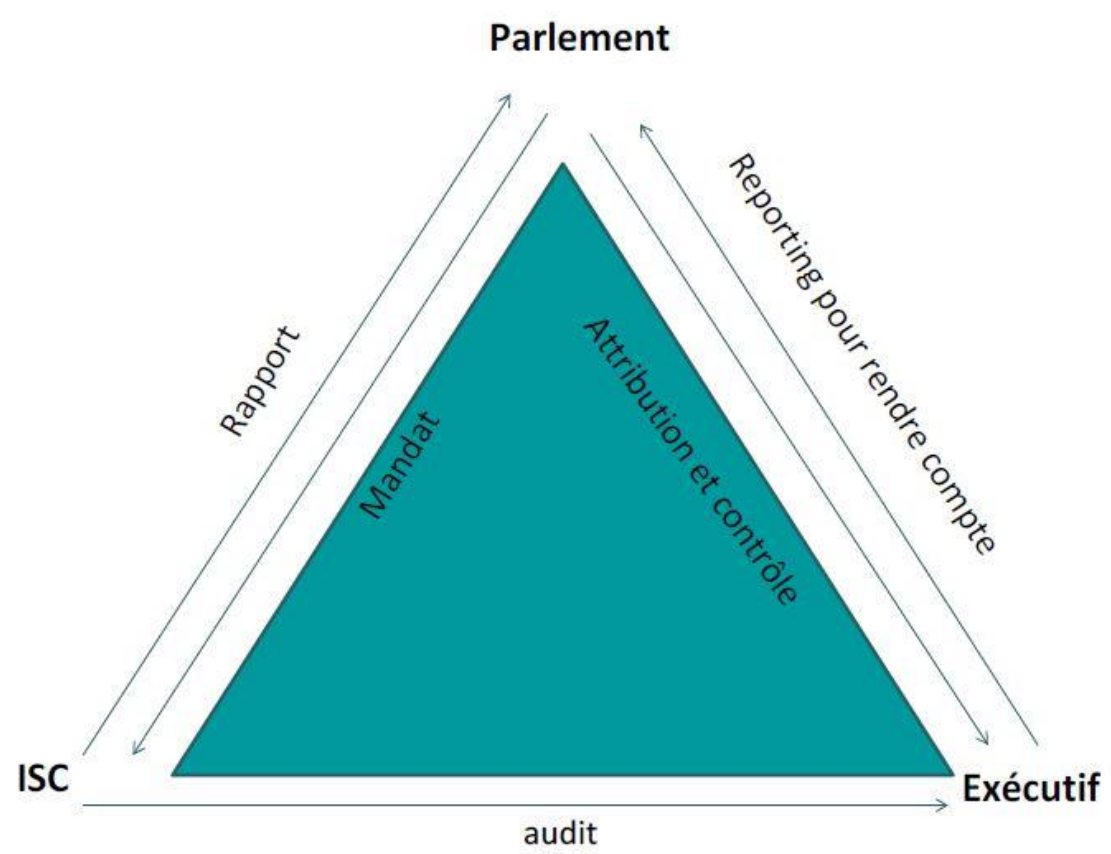

Source : Stapenhurst, Rick et al. (2014), Following the Money: Comparing Parliamentary Public Accounts Committees, London.

Le triangle des responsabilités se base sur le principe selon lequel : le parlement adopte le budget de l'État et autorise l'exécutif à exécuter le budget (attribution) ; l'exécutif soumet au parlement un rapport sur l'exécution du budget; I'institution de contrôle indépendante, conformément à son mandat défini par la loi / Constitution, contrôle l'exécution du budget et présente son rapport au parlement ; et ce parlement, pour le compte des citoyens, oblige l'exécutif à rendre compte (contrôle) et, dans certains pays, octroie une décharge formalisée. Toutes les parties du triangle doivent jouer leur rôle afin de contribuer à une utilisation efficace et responsable des ressources publiques.

Les parlements sont chargés de surveiller l'exécution du budget qu'ils ont adopté. Cette tâche émane du mandat accordé au parlement par les électeurs. Le parlement a la responsabilité de s'assurer que l'exécutif utilise les ressources dans un souci d'efficacité, d'efficience et d'économie, et conformément aux autorisations budgétaires, en s'appuyant sur les travaux de I'ISC. Compter sur l'exécutif pour réagir avec efficacité aux constatations et aux recommandations de I'ISC ne suffit pas car cela équivaudrait à renoncer à la mission fondamentale du parlement, à savoir contrôler l'exécutif.

Pour remplir leur fonction au sein du système de contrôle de la gestion publique, il est important que les ISC veillent à ce que leurs travaux soient pertinents, qu'ils ajoutent de la valeur et qu'ils aient un impact, pas uniquement par le biais d'analyses et de rapports sur les faits passés, mais également en se tournant vers l'avenir, en identifiant les points sur lesquels des améliorations sont possibles et en encourageant les bonnes pratiques. De cette manière, l'audit du secteur public contribue à l'amélioration des normes de gouvernance, du management et du processus de décision, ainsi qu'à une utilisation plus efficace des deniers publics.

Les ISSAl, tout comme d'autres normes et principes, fournissent un cadre et des orientations aux ISC. Elles couvrent ce que les ISC devraient ou pourraient prendre en compte lorsqu'elles analysent les 
possibilités de créer un impact et de le renforcer, et dans leur coopération avec le parlement. Le restant de ce chapitre décrit les attentes majeures, définies par des normes et des directives qui régissent les rapports et les activités des ISC avec les parlements. Le chapitre suivant aborde les actions attendues des parlements en matière de traitement des rapports et de la communication avec les ISC. Les exigences détaillées énoncées dans les normes et les principes sont exposées à l'Annexe 1.

\subsection{Actions attendues des ISC}

La mission de I'ISC est d'établir des rapports et fournir des garanties sur l'utilisation des ressources publiques par le gouvernement, et de la sorte, de contribuer au bon fonctionnement du système de contrôle de la gestion publique. Comme cela a été décrit dans la section précédente, la relation de l'ISC avec le parlement constitue une composante fondamentale de l'efficacité de sa mission, pour développer l'influence de ses activités et renforcer l'obligation de rendre compte. En parcourant les ISSAI et les autres normes pertinentes, on relève certains principes et exigences que I'ISC doit respecter afin de se conformer aux normes professionnelles des ISC. Le tableau 1 analyse les exigences essentielles qu'une ISC doit remplir dans ses relations avec le parlement.

Tableau 1. Normes et principes destinés aux ISC et visant à développer des relations efficaces avec le parlement

\begin{tabular}{|c|c|c|}
\hline & Exigences & Référence \\
\hline \multirow[t]{4}{*}{$\begin{array}{l}\text { Établissement } \\
\text { de rapports }\end{array}$} & Les rapports sont soumis au parlement et publiés. & $\begin{array}{l}\text { ISSAI } 1 \text { Section } 16 \\
\text { ISSAI } 10 \text { Principe } 7 \\
\text { ISSAI } 12 \text { Principes } \\
2,3,4 \\
\text { ISSAI } 20 \text { Principe } 7 \\
\text { les principes de } \\
\text { I'administration } \\
\text { publique, Principes } \\
15,16^{4}\end{array}$ \\
\hline & $\begin{array}{l}\text { Des outils de communication adéquats sont utilisés pour simplifier l'accès aux } \\
\text { rapports et favoriser leur compréhension. }\end{array}$ & $\begin{array}{l}\text { ISSAI } 12 \text { Principe } 4 \\
\text { ISSAI } 20 \text { Principe } 8\end{array}$ \\
\hline & Offre aux législateurs des informations objectives et en temps voulu. & ISSAI 12 Principe 3 \\
\hline & $\begin{array}{l}\text { Les rapports d'audit distinguent les éléments suivants : sujets, constatations } \\
\text { récurrentes, tendances, causes primaires et recommandations d'audit. Ces } \\
\text { éléments sont débattus avec les principales parties prenantes. }\end{array}$ & ISSAI 12 Principe 3 \\
\hline
\end{tabular}

OECD (2014), Les Principes de l'administration publique, OECD, Paris, http://www.sigmaweb.org/publications/Principles-Public-Administration-Nov2014.pdf 


\begin{tabular}{|c|c|c|}
\hline \multirow{4}{*}{$\begin{array}{l}\text { Rôle de } \\
\text { communication } \\
\text { et travaux de } \\
\text { I'ISC }\end{array}$} & $\begin{array}{l}\text { Création de bonnes relations de travail, et de bonnes politiques et procédures de } \\
\text { communication, en lien avec le parlement. }\end{array}$ & \multirow{4}{*}{$\begin{array}{l}\text { ISSAI } 12 \text { Principe } 3 \\
\text { ISSAI } 20 \text { Principe } 7\end{array}$} \\
\hline & Coopère avec le parlement et ses commissions, et sensibilise au rôle de l'ISC. & \\
\hline & Aide le parlement à comprendre les rapports d'audit. & \\
\hline & $\begin{array}{l}\text { Offre des conseils sur les manières d'utiliser au mieux les constatations et les } \\
\text { opinions formulées dans les audits. }\end{array}$ & \\
\hline \multirow{3}{*}{$\begin{array}{l}\text { Communication } \\
\text { intéressant les } \\
\text { parties } \\
\text { prenantes }\end{array}$} & $\begin{array}{l}\text { Connaît les attentes des parties prenantes et est réceptif à leurs avis, sans } \\
\text { compromettre l'indépendance. }\end{array}$ & ISSAI 12 Principe 5 \\
\hline & $\begin{array}{l}\text { S'assure que les attentes et les avis des parties prenantes sont inscrits dans des } \\
\text { plannings organisationnels et de contrôle. }\end{array}$ & ISSAI 12 Principe 5 \\
\hline & $\begin{array}{l}\text { Sonde si les parties prenantes estiment qu'elles sont efficaces et contribuent à } \\
\text { l'amélioration du secteur public. }\end{array}$ & ISSAI 12 Principe 7 \\
\hline \multirow[t]{3}{*}{$\begin{array}{l}\text { Autres types de } \\
\text { communication }\end{array}$} & $\begin{array}{l}\text { La communication contribue à sensibiliser les parties prenantes à la nécessité de } \\
\text { transparence et de responsabilisation du secteur public. }\end{array}$ & ISSAI 12 Principe 3 \\
\hline & Évalue périodiquement l'efficacité de la communication de l'ISC. & ISSAI 12 Principe 3 \\
\hline & Participation au débat portant sur l'amélioration du secteur public. & ISSAI 12 Principe 7 \\
\hline \multirow[t]{2}{*}{ Suivi } & Soumission de rapports de suivi au parlement. & $\begin{array}{l}\text { ISSAI } 10 \text { Principe } 7 \\
\text { ISSAI } 12 \text { Principe } 3 \\
\text { ISSAI } 20 \text { Principes } \\
3,7\end{array}$ \\
\hline & Pilotage et suivi des recommandations faites par l'ISC et le parlement. & $\begin{array}{l}\text { ISSAI } 10 \text { Principe } 7 \\
\text { ISSAI } 20 \text { Principe } 3 \\
\text { Les principes de } \\
\text { I'administration } \\
\text { publique, Principe } 16\end{array}$ \\
\hline $\begin{array}{l}\text { Établissement } \\
\text { de rapport de }\end{array}$ & Soumission d'un rapport d'activité annuel au parlement. & ISSAI 10 Principe 3 \\
\hline
\end{tabular}




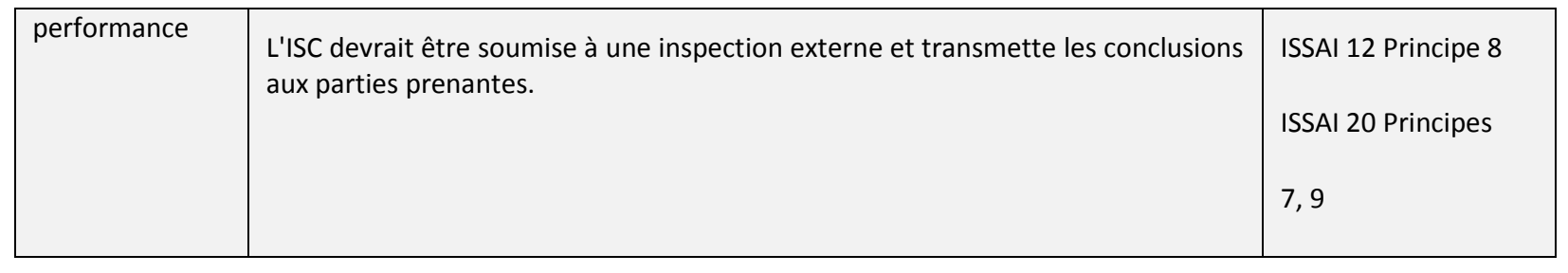

\subsubsection{Rapports adressés au parlement -fondement du lien avec le parlement}

Les principes fondateurs de la mission des ISC en vertu de la norme ISSAI $1^{5}$ (la Déclaration de Lima) et les critères préalables à l'indépendance des ISC définis dans la norme ISSAI $10^{6}$ (la Déclaration de Mexico sur l'indépendance des ISC) formulent les attentes / exigences sous-jacentes au développement d'une relation efficace entre l'ISC et le parlement.

Approuvée en 1977, la Déclaration de Lima a défini depuis de nombreuses années les attentes à la base des relations des ISC avec leur parlement. La Section 16 de la Déclaration de Lima affirme que " la Constitution doit autoriser et obliger l'Institution supérieure de contrôle des finances publiques à présenter des rapports au parlement ; ces rapports doivent être publiés ». Elle affirme en outre que la publication des rapports fournira davantage d'opportunités de faire respecter les constatations des ISC. Cette disposition a été renforcée par la norme ISSAI 10, approuvée par le Congrès international des Institutions supérieures de contrôle (INCOSAI) ${ }^{7}$ en 2007. Le principe 7 de la norme ISSAI 10 déclare que les ISC doivent soumettre des rapports au parlement ${ }^{8}$ pour qu'il les examine et qu'il effectue un suivi des recommandations exigeant des mesures correctives ; que les ISC doivent posséder leur propre système interne de suivi pour veiller à ce que les entités contrôlées donnent suite de façon satisfaisante à leurs observations et à leurs recommandations, y compris à celles du parlement ; que les ISC doivent soumettre leurs rapports de suivi au parlement afin qu'il les examine et prenne des mesures.

Par conséquent, les normes ISSAI 1 et ISSAI 10 définissent le contexte de la relation fondamentale que les ISC doivent entretenir avec le parlement. Par la suite, les ISSAI ont évolué pour fournir d'autres orientations et lignes directrices sur divers aspects de cette relation et sur les actions attendues des ISC pour développer et entretenir cette relation. La norme ISSAI $12^{9}$ (La valeur et les avantages des institutions supérieures de contrôle) et la norme ISSAI $20^{10}$ (Principes de transparence et de

ISSAI 1, La Déclaration de Lima (adoptée en 1977), disponible sur http://www.issai.org/en us/site-issai/issaiframework.

ISSAI 10, La Déclaration de Mexico sur l'indépendance des ISC (adoptée en 2007), disponible sur http://www.issai.org/en us/site-issai/issai-framework/2-prerequisites-for-the-functioning-of-sais.htm.

Qui se tient tous les trois ans.

Bien qu'il y ait des différences entre les concepts de " législature " et de " parlement ", dans le cadre de ce document, nous utiliserons ces termes de manière interchangeable. Laver, M. (2008), Legislatures and parliaments in comparative context, in Weingast, B. and Wittman, D. (eds), Oxford Handbook of Political Economy, Oxford University Press, Oxford.

ISSAI 12, La valeur et les avantages des institutions supérieures de contrôle des finances publiques - faire une différence dans la vie des citoyens (adoptée en 2013), disponible sur http://www.issai.org/en us/siteissai/issai-framework/2-prerequisites-for-the-functioning-of-sais.htm.

ISSAI 20, Principes de responsabilité et de transparence (adoptée en 2010), disponible sur http://www.issai.org/en us/site-issai/issai-framework/2-prerequisites-for-the-functioning-of-sais.htm. 
responsabilité) explicitent encore davantage les attentes, ou les exigences que doivent remplir les ISC au regard de leurs relations avec le parlement (et les autres parties prenantes).

En ce qui concerne la soumission de leurs rapports au parlement, la norme ISSAI 12 déclare par exemple que les "ISC devront, en vertu de leurs mandats, soumettre les rapports de vérification comptable à l'assemblée législative ou tout autre organe public responsable, selon le cas ».

Les normes définissent en outre les attentes portant sur la qualité des informations fournies par les ISC au parlement. Elles indiquent que ces informations doivent être pertinentes, objectives, et soumises en temps opportun. Elles déclarent également que les rapports doivent mettre en lumière les thématiques, les constatations récurrentes, les tendances, les causes primaires et les recommandations. Elles encouragent également les ISC à donner des conseils sur les constatations de contrôle de manière à ce qu'elles soient exploitées au mieux, par exemple en les orientant sur les bonnes pratiques.

Les principes de l'administration publique (2014), élaborés par SIGMA en partenariat avec la Commission européenne (CE), énoncent ce qu'est concrètement une bonne gouvernance ainsi que les exigences majeures imposées aux administrations publiques nationales pendant leur processus d'intégration dans I'UE. En matière de gestion et de contrôle des finances publiques, ces principes répondent aux exigences du chapitre 32 des acquis de I'UE. En vertu du sous-principe 15.8, ils formulent ce qui est fondamentalement attendu de la relation entre I'ISC et le parlement : "L'Institution supérieure de contrôle des finances publiques est habilitée et tenue par la Constitution de présenter annuellement des rapports indépendants au parlement ou à tout autre organe public responsable ; ces rapports doivent être publiés. »

Pour terminer, I'un des axes du Cadre d'évaluation de la gestion des finances publiques (PEFA ${ }^{11}$ ) concerne le contrôle externe et la mission des ISC. L'un des quatre domaines majeurs évalués est la soumission de rapports au parlement.

\subsubsection{Communication - comprendre le rôle et la mission des ISC}

Fondées sur les attentes sous-jacentes établies dans les déclarations de Lima et de Mexico, les ISSAI attendent d'une ISC qu'elle mette en œuvre des modalités de communication et des relations efficaces avec le parlement et ses commissions de supervision concernées. L'ISSAI 12 indique précisément que " les ISC devront développer des relations professionnelles avec les commissions de supervision législatives ainsi qu'avec les directions et conseils d'administration des organismes contrôlés en vue de les aider à mieux comprendre les rapports de contrôle et recommandations et prendre les mesures qui s'imposent. " Ceci est repris dans I'ISSAI 20 qui déclare que "les ISC doivent entretenir d'étroites relations avec les commissions parlementaires concernées afin de les aider à mieux comprendre les rapports et les conclusions d'audit et à prendre les mesures appropriées. »

En conséquence, il est attendu des ISC qu'elles développent leurs relations avec le parlement de telle sorte qu'il comprenne leur rôle et leurs rapports d'audit. Ceci indique avec clarté qu'aujourd'hui, en vertu des normes, la soumission officielle des rapports ne suffit pas pour susciter le suivi et les actions adéquats. Les ISC doivent en faire davantage pour expliquer leur rôle et leurs travaux; elles doivent fournir des notes d'information sur leurs rapports et autres sujets; elles doivent être proactives dans la 
présentation de leur mission et de leurs activités. Elles doivent concevoir et mettre en application une stratégie de communication efficace pour s'assurer que leurs travaux auront un impact, et pour favoriser la bonne gouvernance. Comme le formule I'ISSAI 12, les ISC doivent communiquer de manière à :

- améliorer la connaissance et la compréhension par les parties prenantes des rôles et responsabilités des ISC en tant que contrôleurs indépendants des finances du secteur public ;

- contribuer à la prise de conscience par les parties prenantes du besoin de transparence et de reddition des comptes dans le secteur public ;

- assurer la compréhension du travail de contrôle des ISC et de ses résultats.

La norme ISSAI $21^{12}$ présente quelques exemples de bonnes pratiques : présenter les constatations des rapports aux commissions parlementaires, offrir une orientation et une formation en gestion financière aux parlementaires, fournir un guide sur l'examen des dépenses publiques aux parlementaires, organiser des réunions informelles avec les présidents et les membres des commissions parlementaires afin de leur expliquer le rôle et le mandat de I'ISC et d'être en retour informées sur les besoins des diverses commissions.

\subsubsection{Communication - pertinence pour les parties prenantes}

Au vu de la mission des ISC et pour que leur travail ajoute de la valeur et ait un impact, les ISSAI soulignent expressément l'importance de la pertinence de leurs travaux vis-à-vis du parlement, des citoyens et des autres parties prenantes. La norme ISSAI 12 (La valeur et les avantages des institutions supérieures de contrôle des finances publiques - faire une différence dans la vie des citoyens) propose une ligne directrice qui fait autorité :

Les ISC peuvent témoigner de leur pertinence en répondant de manière appropriée aux exigences des citoyens, aux attentes des différentes parties prenantes et aux risques émergents et environnements changeants au sein desquels les audits sont menés. En outre, il est crucial que les ISC expliquent, de manière positive et efficace, aux parties prenantes dans quelle mesure leur travail contribue à améliorer le secteur public, et ce pour qu'elles soient une source crédible d'informations indépendantes et objectives, qui fait évoluer le secteur public dans le bon sens.

À cet égard, le principe 5 (Savoir répondre aux environnements évolutifs et aux risques émergents), le principe 6 (Communiquer efficacement avec les parties prenantes) et le principe 7 (Devenir une source crédible d'informations indépendantes et objectives et lignes directrices) de I'ISSAI 12 formulent clairement les attentes imposées aux ISC en matière de pertinence de leurs travaux. Ces attentes sont les suivantes :

- Prendre conscience des attentes des parties prenantes et leur répondre ;

- Répondre de manière adaptée aux questions-clés ayant un impact sur la société lorsqu'elles élaborent leur programme de travail ; 
- Évaluer l'évolution et l'émergence des risques dans l'environnement de contrôle et y répondre en temps opportun ;

- S'assurer que les attentes des parties prenantes et les risques émergents soient pris en compte dans les plans stratégiques, les plans de développement et les programmes d'audit, selon le cas ;

- Coopérer avec les parties prenantes et tenir compte de leurs points de vue sans compromettre leur indépendance ;

- Vérifier si les parties prenantes jugent que I'ISC est efficace et contribue à améliorer le secteur public.

S'il est important que I'ISC soit libre de concevoir et de déterminer son programme de travail en toute indépendance, il n'en reste pas moins que sa coopération avec le parlement et les autres parties prenantes est essentiel. Lorsqu'elle planifie et évalue le champ de ses contrôles, cela lui permet d'identifier les problématiques cruciales ayant une incidence sur les services publics, et d'élaborer un programme qui présente une pertinence et un intérêt pour le parlement et les autres parties prenantes. Cette coopération, associée à des processus internes rigoureux d'identification de thématiques de contrôle pertinents, devrait minimiser le risque que des problématiques cruciales liées au service public soient laissées de côté, augmenter la probabilité que les travaux de I'ISC aient un impact, et contribuer à l'élaboration d'un programme de travail pertinent et intéressant.

La coopération de l'ISC avec le parlement et les autres parties prenantes sur son programme de travail, ou l'examen de demandes d'audit, suscite parfois la crainte que l'indépendance de I'ISC soit d'une certaine manière compromise. Toutefois, tant que la faculté de décider du choix de ses audits ou de son programme de travail revient en dernier ressort à I'ISC, et qu'elle dispose de cette latitude pour examiner des thèmes d'audit qui lui sont suggérés, sa coopération avec le parlement et les autres parties prenantes ne devrait pas être vue comme une menace à l'encontre de son indépendance.

Par conséquent, I'ISSAI 12 formule une attente claire selon laquelle l'ISC doit travailler efficacement avec le parlement (et les autres parties prenantes) pour s'assurer que les attentes et les points de vue du parlement soient compris, que le parlement soit sensibilisé aux enjeux auxquels peut être confrontée I'ISC et que tous ces éléments soient pris en compte par I'ISC dans la planification de son travail de contrôle.

Pour entretenir la communication et préserver la pertinence de ses travaux pour les parties prenantes, les normes précisent également que les ISC devraient :

- Contribuer au débat sur les améliorations dans le secteur public, sans compromettre leur indépendance ;

- Faciliter l'accès à leurs rapports par l'ensemble des parties prenantes en utilisant les outils de communication appropriés ;

- Établir des politiques et procédures de communication en liaison avec le parlement ;

- Vérifier régulièrement si les parties prenantes jugent que la communication de l'ISC est efficace.

\subsubsection{Suivi}

Suivant les normes, il est attendu des ISC qu'elles assurent le suivi des observations et des recommandations contenues dans leurs rapports, et qu'elles communiquent leurs résultats. Les normes 
ISSAI 10, 12 et 20 font expressément référence au suivi. Elles prévoient en outre que l'ISC assure le suivi des recommandations faites par le parlement, une de ses commissions, ou le conseil d'administration de l'entité contrôlée, selon le cas.

Les responsabilités en matière de communication qui incombent aux ISC comportent donc l'obligation de rendre compte devant le parlement (et auprès des autres parties prenantes) des résultats de leur travail, afin de vérifier si le gouvernement ou ses organes ont pris les mesures appropriées à la suite des recommandations de I'ISC.

Le suivi efficace des rapports de I'ISC est également un domaine étudié dans le cadre PEFA, au titre des contrôles externes, la note obtenue dépendant de l'efficacité et de la rapidité du suivi réalisés sur les résultats des rapports de I'ISC.

\subsubsection{Rendre compte de la performance des ISC}

Les ISC doivent rendre compte de leur propre performance et de leur utilisation des deniers publics. Les normes définissent des attentes concernant la vérification et la communication de la performance de I'ISC. Ces attentes imposent à I'ISC de remettre des rapports sur sa performance au parlement et aux autres parties prenantes, y compris les états financiers, et de soumettre ces rapports à un contrôle ou un examen externe, les conclusions étant également transmises aux parties prenantes. À titre d'exemple, la norme ISSAI 10 déclare que "les Institutions supérieures de contrôle des finances publiques devraient soumettre un rapport d'activité annuel au parlement .... » et I'ISSAI 12 stipule que " les ISC devront faire l'objet d'un examen externe indépendant, y compris une vérification externe de leurs opérations, et mettre ces rapports à la disposition des parties prenantes. »

Les normes formulent également des attentes qui convient les ISC à vérifier si les parties prenantes jugent qu'elles sont efficaces, si elles contribuent à améliorer le secteur public, et si elles communiquent avec efficacité. Les ISC doivent par conséquent coopérer avec le parlement et le consulter pour s'assurer qu'elles répondent bien à ces attentes.

Pour terminer, en ce qui concerne la performance des ISC, le Groupe de travail de l'INTOSAI sur la valeur et les avantages des ISC a mis au point un cadre de mesure du rendement $(P M F)^{13}$ qui comporte un volet traitant de la communication avec le législatif, l'exécutif et le judiciaire. Ce cadre a été testé dans plusieurs régions de I'INTOSAI. La version définitive a été adoptée durant I'INCOSAI 2016 à Abu Dhabi. Le PMF définit les éléments de bonnes pratiques sur la communication avec le parlement, par rapport auxquelles le rendement de I'ISC peut être mesuré. Elles portent sur les domaines mis en évidence dans la présente section, et suivent dans leur ensemble les normes ISSAI. L'Annexe 2 donne des informations détaillées à ce sujet.

\subsection{Observations finales}

Les attentes imposées aux ISC dans le cadre de leur coopération avec le parlement sont clairement définies par les normes ISSAI et les autres documents d'orientation connexes, afin qu'elles fournissent une garantie et rendent compte de l'utilisation des ressources publiques par le gouvernement et, suite à cela, qu'elles contribuent au bon fonctionnement du système de contrôle de la gestion publique. 
Selon certains, les attentes définies dans l'ensemble des normes ISSAI seraient élevées, et elles incarneraient ce que l'on serait en droit d'attendre des ISC dans les pays très développés, jouissant de démocraties anciennes. Toutefois, cette constatation ne diminue pas la pertinence des attentes envers toutes les ISC car elles devraient aspirer à exécuter leur mandat de manière professionnelle, en ayant une influence croissante. Et quant aux pays qui souhaitent intégrer l'UE, ces attentes correspondent à ce qu'ils devraient s'efforcer de mettre en pratique, en les ajustant en fonction de la situation particulière à chaque pays. 


\section{ATTENTES ENVERS LES PARLEMENTS}

Sous réserve des dispositions légales existant dans certains pays, les parlements sont en principe souverains dans le traitement des rapports des ISC. C'est pourquoi ils font l'objet de beaucoup moins de normes et de principes sur les procédures de traitement des rapports des ISC et sur leurs activités de coopération avec ces dernières. En conséquence, lorsque les ISC demandent au parlement d'accorder l'attention requise à leurs rapports dans le but de renforcer leur impact, elles n'ont pas à leur disposition un ensemble de principes qui font autorité. Toutefois, les Principes de l'administration publique, en relation avec les exigences du chapitre 32 de l'acquis et avec le Cadre d'évaluation de la gestion des finances publiques $P E F A^{14}$, fournissent des principes-clés qui constituent un bon point de départ.

\subsection{Mécanismes officiels dédiés au traitement des rapports des ISC}

Ces documents visent essentiellement à offrir aux parlements une procédure formelle lorsqu'ils étudient les rapports des ISC, ceci afin que les parlements accordent l'attention voulue aux rapports, pour mettre le gouvernement devant son obligation de rendre compte. Ces deux attentes peuvent être considérées comme étant des principes minimum généralement reconnus.

Le cadre PEFA élargit cette attente, en évoquant :

- L'examen des rapports d'audit par le parlement en temps opportun ;

- Le dimensionnement des auditions publiques tenues par le parlement sur les principales constatations des audits;

- La formulation par le parlement d'actions recommandées;

- La mise en œuvre par l'exécutif et le suivi systématique par le parlement.

II n'existe que très peu d'autres normes applicables. Aucune norme officielle ne fait référence à l'existence d'une commission spécifiquement dédiée au traitement des rapports des ISC et chargée de la communication au jour le jour avec les ISC, sans parler d'une commission d'audit parlementaire, telle qu'une commission des comptes publics. II est néanmoins souvent admis que ce type de commission spécialisée constitue un avantage pour traiter efficacement les rapports des ISC. Au final, cette activité dépend de la manière dont le parlement organise son travail et ses procédures, y compris sa façon de déléguer une partie de son travail à ses commissions.

Bien que les orientations proposées par les normes soient relativement limitées, il existe plusieurs documents de recherche concernant la surveillance financière exercée par les parlements ${ }^{15}$. Ces documents fournissent des lignes directrices supplémentaires sur ce qui pourrait être considéré comme des bonnes pratiques de surveillance parlementaire des finances publiques. Selon les bonnes pratiques, toute commission spécialisée devrait avoir les caractéristiques suivantes :

PEFA (2016), Cadre d'évaluation de la gestion des finances publiques, Secrétariat PEFA, Washington, D.C., https://pefa.org/sites/default/files/PEFA\%20Framework English.pdf. 
- Avoir un mandat clairement défini, avec un vaste périmètre ;

- Avoir un personnel et des ressources suffisantes pour assurer son activité ;

- Elle devrait tenir des réunions publiques ;

- Le parlement devrait établir des procédures de suivi claires sur les rapports d'audit, venant en complément de celles de I'ISC ;

- Il conviendrait d'offrir une formation aux membres de la commission, au début et tout au long de leur mandat.

D'autres études offrent des orientations complémentaires sur les bonnes pratiques s'adressant spécifiquement aux commissions d'audit parlementaires.

\subsection{Attentes envers les commissions d'audit parlementaires (PAC)}

Une commission des comptes ou d'audit parlementaire constitue l'une des méthodes que peuvent choisir les parlements pour traiter les rapports des ISC. En dehors des dispositions fixées au niveau national, y compris les dispositions constitutionnelles, les lois parlementaires ou les règlements intérieurs des parlements, il n'existe pas de conventions européennes ou internationales contraignantes qui organiseraient les relations entre les PAC et les ISC. Les parlements jouissent d'un pouvoir discrétionnaire considérable dans les modalités de leur coopération avec les ISC. Il existe néanmoins des études comparatives ${ }^{16}$ qui montrent clairement la diversité des formules institutionnelles concernant les PAC dans les différents pays et, facteur encore plus important dans ce contexte, des méthodes de coopération avec les ISC.

Bien qu'il n'y ait pas de source unique faisant autorité qui résumerait les "bonnes pratiques » parlementaires visant à structurer la relation entre une PAC et une ISC, il est possible de distinguer un ensemble fondamental de principes opérationnels largement acceptés. Ces derniers émergent des études comparatives, mais également d'une variété de documents pertinents, dont :

- Les Principes de l'administration publique élaborés par SIGMA, concernant notamment la gestion des finances publiques (essentiellement le principe 16), en relation avec les exigences du chapitre 32 de l'acquis ;

- Le Cadre d'évaluation de la gestion des finances publiques ${ }^{17}$ PEFA 2016 dont le pilier VII énonce les indicateurs de qualité applicable à l'examen législatif des rapports d'audit ;

- Le document SIGMA $n^{\circ} 332002$ sur les Relations entre les ISC et les commissions parlementaires ${ }^{18}$;

Scottish Parliament (2003), Parliamentary Audit: The Audit Committee in Comparative Perspective, or Stapenhurst, Rick et al. (2014), Following the Money: Comparing Parliamentary Public Accounts Committees, Pluto Press, London.

PEFA (2016), Cadre d'évaluation de la gestion des finances publiques,_Secrétariat du PEFA, Washington, D.C., https://pefa.org/sites/default/files/PEFA\%20Framework_English.pdf

18

OECD (2002), Relations entre les cours des comptes et les commissions parlementaires, Document SIGMA

$\mathrm{N}^{\circ} 33$, OECD Publishing, Paris, http://dx.doi.org/10.1787/5kml60vd5x8r-en. 
- Le document 2015 Coopération pour la responsabilité : Boîte à outils de communication destinée aux commissions des comptes publics et aux institutions supérieures de contrôle publiée par la Deutsche Gesellschaft für Internationale Zusammenarbeit (GIZ) $\mathrm{GmbH}^{19}$ allemande pour le compte de I'Union européenne et du Ministère fédéral allemand de la coopération et du développement économique ${ }^{20}$.

Les exigences et les recommandations formulées dans ces documents et pertinentes dans le cadre du présent document énoncent des préoccupations substantielles dans deux grands domaines: 1) les pouvoirs et les ressources des PAC; et 2) la manière dont les PAC traitent les informations transmises par les ISC.

\subsubsection{Pouvoirs et ressources des PAC}

Les droits juridiques, les prérogatives et les effectifs des PAC sont d'une importance décisive quant à leur capacité à profiter au mieux des informations fournies par les ISC et à contribuer efficacement à la mission d'examen, de surveillance et de contrôle de la gestion publique du parlement dans son ensemble. Stapenhurst et al. recommandent entre autres aux PAC :

- d'avoir entre 5 et 11 membres,

- d'être présidées par un membre d'un parti d'opposition,

- d'être nommées pour la durée de la législature,

- d'avoir un mandat et des pouvoirs clairement définis, pour s'assurer de la mise en application des recommandations,

- de travailler de manière non partisane,

- d'avoir les effectifs adéquats, avec un secrétariat qualifié et un personnel compétent ayant la formation appropriée et l'accès aux savoir-faire requis,

- de solliciter l'expertise et le conseil de l'ISC à l'occasion de toutes ses délibérations.

De même, le document SIGMA de 2002 suggère qu'une PAC devrait, dans la mesure du possible :

- agir d'une " façon apolitique ",

- avoir un membre d'un parti d'opposition au poste de président de la commission,

- avoir des effectifs suffisants, avec éventuellement le détachement d'au moins un membre du personnel de l'ISC pour assister la commission dans l'exécution de ses rapports,

- tenir le parlement dans son ensemble parfaitement informé de ses constatations,

- demander au parlement de soutenir ses constatations et ses recommandations,

- s'assurer que ses propres rapports et recommandations soient élaborés en temps opportun afin d'obliger le gouvernement à rendre compte. 
Le Cadre PEFA stipule " qu'll importe que les résultats de cet examen des rapports d'audit externe par tout comité mandaté à cet effet soient présentés pour examen (et dans l'idéal fassent l'objet d'une audition) en séance plénière de l'assemblée législative pour constituer un examen à part entière » (p. 83).

En substance, le premier groupe de principes opérationnels vise à permettre aux PAC de bénéficier au maximum de l'expertise offerte par les ISC. Les PAC doivent avoir les moyens de contribuer pleinement à la fonction de contrôle de la gestion publique qui incombe au parlement dans son ensemble, et de favoriser la réalisation d'actions correctives par le gouvernement.

Les pouvoirs et les ressources des PAC revêtent une importance critique pour les ISC, car les PAC deviennent les interlocuteurs majeurs (ou points de contact) entre les ISC et le parlement.

\subsubsection{Traitement des informations transmises par l'ISC}

Lorsque les rapports annuels servent de fondement à une décharge, assortie ou non de réserves, (ou bien, dans des cas rares, à un refus de décharge), il est indispensable que les PAC examinent les rapports en temps voulu. Elles doivent débattre des observations et des recommandations faites par I'ISC et rédiger leurs propres rapports en temps voulu, en tenant compte des informations fournies par l'ISC et en présentant clairement les sujets préoccupants; ces règles devraient augmenter les chances que l'exécutif prenne des mesures en réponse aux observations de I'ISC. Comme cela est souligné dans le document SIGMA $n^{\circ} 33$ : "Dans nombre de commissions parlementaires, la pratique veut qu'elles élaborent, dans un délai raisonnable, leur propre rapport sur un rapport d'audit de I'ISC qu'elles ont examiné et qu'elles formulent leurs propres recommandations au gouvernement, d'après les conclusions et recommandations de I'ISC. (...) Les rapports de la commission parlementaire et de l'ISC seraient plus intéressants et facilement acceptables par le gouvernement s'ils incluaient des recommandations axées sur l'avenir » (p. 28).

Dans le chapitre sur la gestion des finances publiques, principe 5, sous-principe 7 , des Principes de l'administration publique, il est recommandé que, avant de débattre du prochain projet de budget, la ou les commissions parlementaires concernées discutent le rapport financier annuel et le rapport correspondant de I'ISC (p. 85).

L'importance de l'examen en temps voulu des rapports d'audit de l'ISC par le parlement est réitérée par le Cadre PEFA qui note que "c'est un facteur essentiel de l'efficacité de la fonction de responsabilisation » et suggère que l'examen parlementaire d'un rapport de I'ISC portant sur le rapport financier annuel intervienne de préférence dans les trois mois suivant sa soumission au parlement. II relève que

Cette ponctualité peut être compromise par un fort accroissement du nombre de rapports d'audit soumis, dû au fait que les contrôleurs externes s'efforcent de rattraper les retards accumulés dans l'examen des dossiers. Dans ce cas, les comités peuvent décider de donner la priorité aux rapports d'audit qui couvrent les dernières périodes auditées et aux entités auditées qui se sont révélées dans le passé ne pas respecter les normes et prescriptions. L'évaluation doit juger favorablement cette manière de procéder qui est conforme aux bonnes pratiques et ne pas prendre en compte les retards qui en résultent pour l'examen des rapports concernant des périodes plus reculées (p. 90).

Les trois autres principes généraux du Cadre PEFA portant sur les méthodes utilisées par les parlements pour traiter les informations fournies par les ISC font référence : 
- à la transparence des informations transmises par les ISC,

- au caractère public des délibérations de la PAC,

- aux interactions directes avec le personnel des ISC.

Le premier principe généralement accepté énonce que toutes les informations fournies par I'ISC au parlement doivent être rendues publiques, sauf si des motifs impérieux l'empêchent.

En second lieu, les PAC en particulier devraient agir de manière transparente chaque fois que cela est possible. Le document SIGMA $n^{\circ} 33$ déclare : "Dans de nombreux pays, il s'est avéré bénéfique de permettre au grand public et aux médias d'assister aux réunions des commissions parlementaires afin d'encourager la transparence et la sensibilisation du grand public aux sujets abordés ${ }^{21}$. Le Cadre PEFA stipule que, idéalement, "toutes les auditions sont ouvertes au public, sauf dans des circonstances strictement déterminées, par exemple les débats ayant trait à la sécurité nationale ou des débats sensibles analogues. Les rapports des comités sont examinés en séance plénière de l'assemblée législative et publiés sur un site web officiel ou par tout autre moyen accessible au public. »

Pour terminer, il est généralement reconnu que, tandis que les documents écrits constituent la source primaire des informations remises par I'ISC au parlement, ce dernier doit s'efforcer de faire participer le personnel de I'ISC à ses débats. Le document SIGMA n 33 est explicite à cet égard lorsqu'il déclare " qu'll est souhaitable que le président ou les cadres supérieurs de l'ISC assistent aux réunions de la commission parlementaire quand des rapports d'audit de ladite ISC doivent y être discutés ${ }^{22}$. " Le Cadre PEFA va dans le même sens lorsqu'il stipule que "Des auditions " approfondies " sur les principales conclusions des rapports d'audit externe ne peuvent être considérées avoir été menées que si elles ont réuni des représentants de I'ISC pour expliquer ses observations ${ }^{23}$. "

\subsection{Observations finales}

Bien que le nombre de principes clairs concernant le suivi par le parlement des examens des rapports remis par les ISC et leur travail avec ces dernières soit très modeste, il est attendu au minimum des parlements qu'ils aient recours à des mécanismes formalisés pour examiner les rapports des ISC, et que ces rapports soient utilisés pour imposer au gouvernement de rendre compte. Il ressort des documents d'analyse des PAC un large consensus sur leurs modalités d'organisation et les ressources dont elles devraient disposer afin de bénéficier au maximum de l'expertise offerte par les ISC. Il existe également un ensemble de principes largement recommandés qui expliquent comment les PAC devraient traiter les informations présentées par les ISC, en particulier s'agissant de leurs rapports d'audit périodiques. Dans leur communication avec les parlements, les ISC peuvent - et doivent - promouvoir l'établissement de dispositions parlementaires relatives au traitement de leurs travaux et, ce faisant, peuvent faire référence à ces attentes minima, ainsi qu'aux bonnes pratiques ressortant des études comparatives.

OECD (2002), Relations entre les Institutions supérieures de contrôle et les commissions parlementaires, Document SIGMA N³3, OCDE, Paris, p. 34, http://dx.doi.org/10.1787/5kml60vd5x8r-en. Idem.

23

PEFA (2016), Cadre d'évaluation de la gestion des finances publiques,_Secrétariat du PEFA, Washington, D.C., p. 83, https://pefa.org/sites/default/files/PEFA\%20Framework English.pdf. 


\section{RELATIONS ENTRE LES ISC ET LE PARLEMENT : QUELQUES FACTEURS CONTEXTUELS}

\subsection{Introduction}

Les normes et principes énoncés dans les chapitres précédents offrent un cadre aux relations entre les ISC et le parlement. La conformité à ce dispositif doit prendre en compte le contexte culturel, juridique et politique de chaque pays. Les différents pays présentent des différences significatives dans l'organisation constitutionnelle et juridique du parlement, de I'ISC, des organes étatiques et du système budgétaire. Tous ces éléments influencent le système de contrôle de la gestion publique et l'efficacité des relations de travail entre les ISC et le parlement. Le présent chapitre aborde quelques facteurs majeurs sous-jacents aux relations entre les ISC et le parlement.

\subsection{Facteurs qui influencent la relation de I'ISC avec le parlement}

\subsubsection{Modèles organisationnels}

Tandis que les ISSAI définissent un cadre objectif destiné à toutes les ISC, ces dernières se sont développées au sein de traditions culturelles, juridiques et politiques propres à leur pays, ce qui a conduit à une grande diversité dans la manière dont elles fonctionnent. Cependant, malgré cette diversité, la majorité des ISC suivent dans les grandes lignes l'un des trois modèles suivants :

- Modèle « Office » / " Auditeur général » (parlementaire / Westminster)

- Modèle juridictionnel (napoléonien)

- Modèle collégial non juridictionnel

Dans le modèle Office/Auditeur général, I'ISC est généralement dirigée par une seule personne (l'auditeur général) qui est parfois un membre du parlement, comme c'est le cas au Royaume Uni (UK), ce qui a pour effet de créer une relation étroite avec le parlement. Le personnel de contrôle a normalement une formation financière et comptable. Traditionnellement, les ISC qui suivent ce modèle se sont centrées sur l'audit financier et, dans les dernières années, sur la performance, et moins sur la conformité aux lois et aux réglementations, bien qu'en pratique, certaines ISC du type Office/Auditeur général établissent des rapports approfondis sur des cas individuels de non-conformité. Dans des situations spécifiques, elles sont dans l'obligation de transmettre le cas au procureur. Elles n'ont généralement pas le pouvoir ou l'autorité d'imposer la mise en œuvre des recommandations ou d'infliger des sanctions à l'encontre des agents publics. Pour avoir un impact, les ISC du modèle Office/Auditeur général dépendent de la force de leurs arguments, de leur crédibilité en tant que source indépendante d'informations objectives, et de leur coopération avec les parties prenantes, en particulier avec le parlement.

Pour que ce modèle soit efficace, I'ISC a besoin, pour amener le gouvernement à rendre compte, du soutien du parlement, qui utilise son expertise. Les travaux de I'ISC ont davantage d'impact lorsque le parlement s'y intéresse activement et s'en sert pour examiner l'utilisation des ressources publiques par le gouvernement et mettre ce dernier devant ses responsabilités. Une telle configuration incite davantage ce dernier à donner suite aux résultats des travaux réalisés par I'ISC. Toutefois, lorsque le parlement ne s'intéresse guère aux activités de l'ISC ou que son examen n'est pas poussé, l'obligation de rendre compte de l'utilisation des finances publiques peut être affaiblie et l'influence de I'ISC limitée. 
Dans le modèle juridictionnel, I'ISC fonctionne comme un tribunal judiciaire. Ses membres sont des magistrats dont le statut est semblable à celui des juges des autres ordres, et le personnel de contrôle a généralement une formation juridique. Historiquement, les travaux de contrôle des ISC du modèle juridictionnel se sont concentrés sur la vérification et le jugement de la légalité des opérations retracées dans les comptes financiers préparés par les comptables publics. Par leurs arrêts, les ISC du modèle juridictionnel statuent sur la légalité des opérations des comptables publics. Elles peuvent soit les décharger de toute responsabilité, soit faire exécuter leurs conclusions et leur imposer une sanction s'ils ont enfreint les règlements régissant la gestion des finances publiques. En règle générale, les ISC du modèle juridictionnel transmettent également au parlement un rapport sur le compte général de l'État pour chaque période budgétaire, à la suite duquel le parlement peut décharger le gouvernement de sa responsabilité pour l'année s'il est satisfait de la gestion des fonds publics. Historiquement, par conséquent, I'ISC oblige les agents publics à rendre compte, la participation du parlement se limitant alors à utiliser le rapport de I'ISC pour octroyer la décharge sur le compte général du budget de l'État. Toutefois, dans la pratique, le mandat de la majorité des ISC du modèle juridictionnel inclut les audits financiers et de performance, ainsi que l'établissement de rapports sur des problématiques systémiques.

Dans le modèle collégial non juridictionnel, I'ISC est gérée par un collège, un conseil/comité ou un groupe d'auditeurs généraux de même rang hiérarchique. Dans certains pays tels que l'Allemagne, les membres du conseil ou du collège ont un statut semblable à celui des magistrats. La nature des missions des ISC du modèle collégial non juridictionnel (et la formation de leur personnel) a généralement été fonction du contexte législatif et historique général du pays dans lequel elles interviennent. Dans certains cas, elles se sont développées vers l'audit financier du modèle Office, comme aux Pays-Bas, ou dans le contexte juridique du modèle juridictionnel, par exemple au Luxembourg. Comme les ISC du modèle Office, elles n'ont généralement pas le pouvoir ou l'autorité d'imposer leurs recommandations ou de prendre des sanctions à l'encontre des agents publics. L'intérêt et le rôle actif joué par le parlement dans l'utilisation de leurs travaux est un facteur-clé quant à l'impact de leur mission.

La figure 3.1 classe les ISC des 28 Pays membres de I'UE, des 7 pays du Réseau y compris I'ISC de I'UE ${ }^{24}$ selon ces catégories générales. Ces groupes ne sont toutefois pas homogènes et il est parfois difficile de classer des ISC dans l'une des trois catégories car elles peuvent présenter les caractéristiques de plusieurs modèles ; en outre, certaines ISC ont évolué et ont changé de modèle. 
Figure 3.1 Nombre d'institutions supérieures de contrôle (ISC) par modèle organisationnel

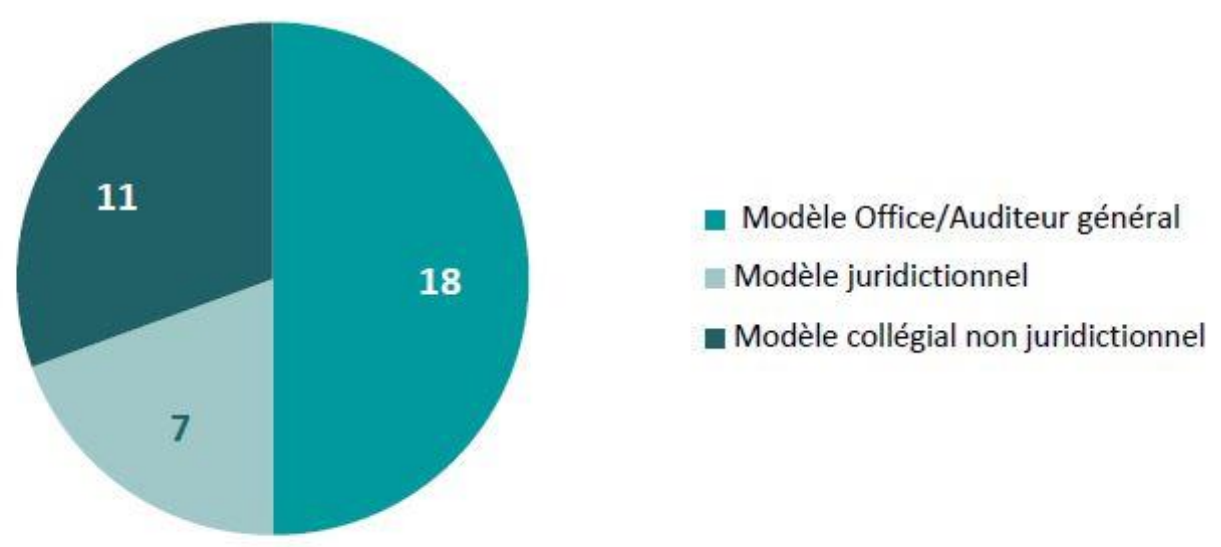

Source : résultats d'études et sites Internet des institutions supérieures de contrôle.

Remarques :

ISC du modèle Office/Auditeur général : Albanie, Autriche*, Bosnie-Herzégovine, Bulgarie*, Croatie, Chypre, Danemark, Estonie, Finlande, Hongrie, Irlande, Kosovo, Lituanie, l'ancienne République yougoslave de Macédoine, Malte, Pologne*, Slovénie*, Royaume-Uni.

ISC du modèle juridictionnel : Belgique, France, Grèce, Italie, Portugal, Espagne, Turquie.

ISC du modèle collégial et non juridictionnel : République tchèque, Cour des comptes européenne (ECA), Allemagne, Lettonie*, Luxembourg, Monténégro, Pays-Bas, Roumanie, Serbie, République slovaque, Suède *.

* ISC dont le classement peut prêter à discussion.

\subsubsection{Mandat de l'ISC}

Comme le montre le schéma ci-dessus, le modèle d'ISC et les traditions du pays jouent un rôle important pour déterminer si le contrôle exercé par l'ISC découle, du point de vue historique, d'une approche axée sur l'audit financier ou d'une approche axée sur la conformité aux lois et règlements, ainsi que la nature de ses relations avec le parlement. Toutefois, bien que les ISC conduisent leurs audits de diverses manières, en mettant l'accent sur des aspects distincts, au fur et à mesure que la communauté des ISC a évolué, et que leurs normes ont été de plus en plus formalisées, un grand nombre d'ISC réalisent désormais des audits financiers, de conformité et de performance.

À titre d'exemple, tandis que les ISC du modèle juridictionnel telles que la Cour des comptes en France exercent toujours leur fonction juridictionnelle en jugeant la légalité des opérations réalisées par les experts-comptables, elles mènent également des audits financiers et de performance, et rendent compte de leurs résultats au parlement. La mission de la Cour des comptes turque (TCA) se concentrait autrefois essentiellement sur la légalité des comptes, mais la nouvelle législation a élargi son champ de compétence, lui permettant de réaliser des audits financiers et de performance.

Dans les modèles Office ou collégial non juridictionnel, les ISC n'ont pas de fonction juridictionnelle relative à la légalité des opérations, mais elles peuvent réaliser malgré tout, dans une certaine mesure, des audits de conformité pour s'assurer que les opérations respectent la réglementation en vigueur. Ces contrôles peuvent être associés à un audit financier, comme en Suède, l'audit financier se limitant à 
examiner la conformité avec les crédits et le droit budgétaire. D'autres, comme l'ECA et l'ISC de Pologne, peuvent être conviées à établir des rapports plus approfondis sur des questions de non-conformité par rapport à la réglementation. De manière générale, elles auront également la responsabilité de signaler les infractions graves aux autorités de police ou au parquet compétent. Dans un petit nombre de pays, comme en Serbie et au Monténégro, I'ISC doit enquêter sur des responsabilités spécifiques et transmettre les rapports aux procureurs sur des questions de non-conformité de faible importance (délits mineurs) ainsi que sur les infractions économiques et les faits délictueux.

Il est souvent assez simple de rendre compte des résultats de l'audit financier, car les audits sont généralement exécutés et les rapports établis suivant des procédures bien structurées, avec, pour objectif, d'exprimer une opinion (sans réserve, avec réserves, défavorable, impossibilité d'exprimer une opinion ${ }^{25}$ ) sur la fiabilité et la crédibilité des informations financières. Cette structure simplifie relativement la tâche du parlement lorsqu'il doit sélectionner les éléments à examiner et se consacrer uniquement aux cas des opinions défavorables, comportant des réserves, ou aux cas d"impossibilité d'exprimer une opinion. Les audits de performance peuvent être plus difficiles à appréhender car l'interprétation des résultats, des conclusions et des recommandations est plus ouverte au débat, et sujette à une sensibilité politique accrue. En conséquence, les audits de performance ont tendance à susciter un plus grand intérêt auprès des parties prenantes, et ils permettent à I'ISC d'accroître son impact, bien que cela comporte des risques. Au Royaume-Uni par exemple, alors que tous les rapports d'audit de I'ISC sont déposés devant le parlement suivant diverses modalités, la commission des comptes publics a tendance à focaliser son attention sur les rapports d'audit de performance, y compris sur des enquêtes spécifiques ; en 2015 , la commission a examiné uniquement les rapports d'audit de performance. L'audit de performance se limite parfois aux indicateurs de performance, comme c'est le cas avec la TCA.

On constate également une grande diversité dans les types d'organismes publics inclus dans le champ de compétence des ISC. Par définition, toutes les ISC examinent les comptes et l'utilisation des ressources du gouvernement central. Mais parmi les ISC des Pays membres de I'UE et du Réseau, il existe différents niveaux d'implication au regard du contrôle des gouvernements régionaux et locaux, des municipalités, des entreprises publiques et des autres organismes es et destinataires de fonds publics $^{26}$. À titre d'exemple, en Autriche, Croatie et Pologne, le mandat de I'ISC s'applique à toutes les strates du gouvernement, aux municipalités, aux entreprises publiques et à tous les autres organes publics. Ce qui signifie que I'ISC doit contrôler ces entités et communiquer les résultats aux assemblées régionales ou locales, ce qui complexifie encore sa tâche.

Dans d'autres pays tels que I'Irlande, la Suède et l'Allemagne, I'ISC concentre son action sur les organes publics et éventuellement les entreprises publiques, ainsi que sur le gouvernement central. Dans ce contexte, les audits au niveau régional ou local peuvent être exécutés par des institutions de contrôle dédiées à ce niveau de gouvernement, ou bien par des cabinets d'audit privés. Au Royaume-Uni par exemple, il existe des dispositions complexes, avec des institutions de contrôle régionales en Écosse, au

ISSAI 1700, Fondement de l'opinion et rapport d'audit sur les états financiers (adoptée en 2010), http://www.issai.org/en us/site-issai/issai-framework/4-auditing-guidelines.htm.

ISSAI 1705, Modifications apportées à l'opinion formulée dans le rapport d'audit indépendant (adoptée en 2010), http://www.issai.org/en us/site-issai/issai-framework/4-auditing-guidelines.htm.

UK National Audit Office (2005), State Audit in the European Union, London, https://www.nao.org.uk/report/state-audit-in-the-european-union/ 
Pays de Galles et en Irlande du Nord chargées de contrôler les gouvernements régionaux et locaux, ainsi que les échelons déconcentrés du gouvernement central dans ces régions; en Angleterre en revanche, le contrôle des autorités locales et municipales est désormais exécuté par le secteur privé après l'abolition récente de la Commission de contrôle, bien que certaines responsabilités d'audit des autorités décentralisées aient été transférées à I'ISC du Royaume-Uni.

\subsubsection{Soumission des rapports au parlement}

Les procédures d'établissement et de soumission des rapports sont étroitement liées à la nature du mandat et au champ de compétence de I'ISC ; c'est un autre facteur important à prendre en compte dans les relations entre les ISC et le parlement. Les rapports envoyés par les ISC au parlement sont de types, de nature et de taille très différents. À titre d'exemple, le nombre de rapports soumis par les ISC se situe entre un et deux, jusqu'à des centaines par an. Les rapports de certaines ISC rassemblent les principales constatations de tous les audits et portant sur toutes les entités contrôlées. D'autres ISC transmettent un rapport par audit, par sujet ou thématique, ou par entité contrôlée. II s'ensuit que le type de coopération nécessaire entre les ISC et le parlement reflètera dans une certaine mesure la nature des procédures d'établissement et de soumission des rapports au parlement.

\subsubsection{Dirigeants et évolution}

Les ISC continuent d'évoluer et de se développer, et les changements au sein d'une ISC sont naturellement susceptibles d'influencer ses relations avec le parlement. Les relations individuelles entre les dirigeants et les agents publics des institutions sont importantes, en particulier la relation entre le chef de l'ISC et le président de la commission parlementaire concernée. Des changements de personnes à ces postes influenceront le travail et l'impact de I'ISC, en particulier dans le modèle Office.

L'environnement de travail de I'ISC peut modifier sa capacité à fournir des prestations pertinentes et intéressantes pour le parlement, influençant ainsi leurs relations. Citons les exemples suivants : le développement des normes de contrôle, le taux de renouvellement et la formation du personnel, les innovations techniques tels que les outils informatiques et de communication (ICT), la disponibilité de volumes de données toujours plus grands et la nécessité de s'y adapter, l'introduction et le développement de l'audit interne, et les autres changements touchant l'environnement de contrôle interne. Une gestion opérationnelle et financière de meilleure qualité au sein des gouvernements devrait en outre aboutir à des résultats plus positifs, par exemple dans les rapports d'audit financiers, et vraisemblablement réduire du même coup l'intérêt du public et des parlements pour ces rapports.

\subsubsection{Rôle joué par le parlement dans l'élaboration du programme de travail de I'ISC}

La capacité du parlement à influencer ou à participer à l'élaboration du programme de travail de I'ISC aura des retombées sur la nature et le type du lien entre I'ISC et le parlement. La plupart des pays ont des dispositions juridiques qui habilitent le parlement à demander à I'ISC d'examiner des problématiques ou des sujets spécifiques. Même en l'absence de dispositions juridiques, les ISC indiquent généralement qu'elles apprécient de recevoir des suggestions ou des requêtes de la part du parlement et des autres parties prenantes. Toutefois, dans pour ainsi dire tous les pays, il est clair qu'en définitive, c'est l'ISC qui décide du contenu de son programme de travail. Par exemple, alors que le Contrôleur et Auditeur général ${ }^{27}$ au Royaume-Uni est absolument souverain dans l'exercice de ses

« Contrôleur et Auditeur général » est le titre du chef de l'ISC du Royaume-Uni. 
fonctions, la loi exige " qu'il prenne en compte toute proposition faite par la commission des comptes publics » lorsqu'il évalue l'opportunité de procéder à un audit de performance. Bien qu'il n'y ait pas de dispositions juridiques obligeant I'ISC à examiner des requêtes émanant d'autres parlementaires ou d'autres commissions, elle examine avec sérieux les questions soulevées et certaines de ces requêtes donnent lieu à des enquêtes complètes et à des rapports sur la performance.

\subsection{Dispositifs parlementaires relatifs à l'examen des travaux des ISC}

Les dispositifs organisationnels présentent de grandes différences, mais les résultats de notre étude indiquent que les dispositifs parlementaires affectés à la surveillance financière comprennent généralement soit une commission spécifique qui s'occupe directement des travaux de I'ISC, soit I'utilisation de la commission budgétaire du parlement. La différence majeure entre ces deux modèles est fondée sur la fonction de surveillance exercée par ces commissions, qui est soit ex ante et ex post, soit ex post $t^{28}$ uniquement. Les commissions de contrôle parlementaires tendent à s'occuper uniquement de la surveillance financière ex post, alors que les commissions budgétaires chargées de l'examen des travaux de I'ISC participent également à la procédure d'adoption des projets de budget, et par conséquent, exercent également la fonction de surveillance ex ante. Naturellement, lorsque la commission n'est pas seulement chargée d'examiner les travaux de l'ISC, elle se concentre et s'intéresse surtout à l'examen du budget de l'État, ce qui lui laissera moins de temps pour l'étude des travaux de I'ISC. Toutefois, dans certains cas où la commission budgétaire a été investie de cette mission, elle a créé une sous-commission spéciale qui fonctionne pleinement en pratique comme une commission de contrôle parlementaire, comme en Serbie.

Les dispositifs susmentionnés sont les plus fréquemment observés, mais il en existe toutefois beaucoup d'autres: au sein du système bicaméral roumain par exemple, il y a habituellement une séance conjointe des commissions des deux chambres qui examine les rapports d'audit et qui prépare un rapport commun à l'intention du Sénat et de la Chambre des députés; en Espagne, une commission conjointe des deux chambres étudie les rapports de I'ISC. La Suède au contraire se démarque nettement des dispositifs décrits ci-dessus car les rapports de I'ISC sont traités par la commission sectorielle ou thématique concernée, et non par une commission spécialisée. En Pologne, les rapports d'audit sont soumis simultanément à la commission de contrôle de l'État et à la commission sectorielle ou thématique concernée.

Dans un nombre toujours croissant de pays, outre le rôle joué par la commission de contrôle ou par la commission budgétaire, les commissions sectorielles ou thématiques utilisent de plus en plus les rapports des ISC qui font l'objet de discussions, en particulier les rapports d'audit de performance qui concernent leur sphère de compétence.

L'organisation au sein des parlements ne correspond pas à un modèle unique "prêt à porter » : Elle dépend de la manière dont le système des commissions fonctionne, s'il est possible de créer des souscommissions, si le parlement dans son ensemble délègue ou non des travaux aux commissions, s'il est possible de travailler en commission mixte, si les débats budgétaires ont lieu uniquement au sein de la 
commission budgétaire ou bien également dans les commissions sectorielles, et si les ministres du gouvernement sont des parlementaires ou non. Dans ce dernier cas, il est d'autant plus important que la commission de contrôle ait un président qui appartienne à l'opposition. Une commission ou une souscommission de contrôle séparée n'accroît pas nécessairement les capacités d'examen des rapports de I'ISC, mais elle peut contribuer à attirer davantage l'attention sur les rapports de I'ISC et à renforcer l'image et l'autorité de I'ISC.

II ne faut pas oublier que les parlementaires ont tendance à s'intéresser davantage à l'avenir (par ex. en matière de politiques, de budget, de législation) que les ISC qui, de par leurs fonctions, s'intéressent à des faits passés dans leurs travaux d'audit. Cette situation est un défi de taille pour les ISC qui tentent d'attirer l'attention du parlement sur leurs travaux. Elle souligne pour les ISC I'importance de fournir, sur la base de leurs audits, des informations de valeur, et des recommandations réalistes et tournées vers l'avenir, afin d'accroître l'intérêt des parlementaires, et par là-même, l'impact potentiel de leurs travaux d'audit. Les ISC devraient réfléchir à la manière dont leurs travaux peuvent être pertinents vis-à-vis du parlement. Parallèlement, les parlements devraient examiner quelle valeur ajoutée I'ISC pourrait apporter à leurs travaux, en plus des instruments qu'ils utilisent dans leurs fonctions de surveillance et de contrôle, comme les investigations et les enquêtes, et des instruments plus traditionnels comme les auditions, les interpellations, et les questions orales ou écrites au gouvernement.

\subsection{Organisation gouvernementale}

Le triangle des responsabilités de la section 1.1 du présent document montre un modèle simplifié des relations directes entre l'exécutif, le parlement et I'ISC, l'exécutif étant considéré comme une entité gouvernementale unique responsable de toutes ses activités devant le parlement. Mais le système de gestion et de contrôle de la gestion publique est en réalité souvent plus compliqué : par exemple si l'organisation de l'exécutif est divisée entre des agences semi-autonomes, les dirigeants de ces organismes sont tenus de rendre compte directement au parlement. Cette situation peut entraîner une participation plus prononcée de I'ISC et du parlement dans la gestion des agences gouvernementales, tout en diminuant l'attention envers des problématiques plus systémiques.

Le triangle des responsabilités montre le parlement également comme une entité unique, alors que dans les faits, le système de contrôle de la gestion publique fonctionne au travers de différentes commissions ou organes de travail, et est parfois partagé entre deux chambres parlementaires. Cette division des tâches au sein du parlement fait souvent écho à l'organisation du gouvernement, en instaurant des commissions permanentes pour tous les ministères, ou tout au moins pour les principaux. Il est important que les ISC réfléchissent à la manière dont les caractéristiques du système de gestion et de contrôle de la gestion publique affectent l'efficacité des circuits de communication et de reddition des comptes.

\subsection{Système budgétaire et comptable}

Un autre facteur susceptible d'influencer les relations entre les ISC et leur parlement est le système budgétaire et comptable. Lorsque le système budgétaire et comptable est caractérisé par une comptabilité de caisse, l'audit conduit par I'ISC sur l'exécution du budget produira des observations différentes de celles résultant d'un audit réalisé sur une comptabilité d'exercice/en droits constatés. De même, le contrôle de lignes budgétaires relativement petites entraînera des observations différentes de celles d'un audit de lignes budgétaires importantes. Un budget d'investissements séparé nécessite une autre approche de contrôle, avec un autre type d'observations. Les audits des budgets fondés sur la performance comporteront automatiquement des indicateurs de performance, élargissant ainsi la 
portée de l'audit annuel et produisant un autre type de rapport. Bien que ces variables n'influencent pas directement les relations entre I'ISC et le parlement, elles ont une incidence sur le type de rapport d'audit soumis au parlement, déterminant éventuellement diverses exigences que le parlement devra prendre en compte dans l'examen de ces rapports.

\subsection{Dynamiques dans un environnement politique}

Les élections parlementaires ont généralement lieu tous les quatre ou cinq ans, parfois plus souvent en fonction des circonstances politiques. Ceci crée déjà un environnement dynamique, avec des changements de députés, de présidents et de membres de commissions spécialisées, obligeant ainsi I'ISC à nouer de nouvelles relations. Les procédures sont susceptibles de changer, et l'intérêt que porte le parlement à certains sujets change avec le temps, tandis que les problématiques-clés qui touchent le pays et son électorat évoluent. L'intérêt du parlement dans les questions de gestion publique fluctue en fonction des problèmes et des enjeux du jour, et le contenu des politiques et des programmes peut bien évidemment présenter davantage d'intérêt pour les hommes politiques que leur mise en œuvre par les agences gouvernementales.

II s'en suit qu'une ISC doit être souple et capable de réagir à l'environnement politique changeant pour s'assurer que son expertise est jugée pertinente, sans compromettre son indépendance.

\subsection{Observations finales}

Les relations entre I'ISC et le parlement sont gouvernées par un grand nombre de facteurs, qui vont du contexte constitutionnel et juridique jusqu'à la relation personnelle entre le chef de I'ISC et le président du parlement ou le président de la commission spécialisée. Par ailleurs, elles peuvent être influencées par des rivalités internes au parlement ou par des désaccords internes à I'ISC. Les relations entre les ISC et leur parlement sont continuellement influencées par ces facteurs ; la capacité à être souple, adaptable et réactif aux changements de circonstances est indispensable pour entretenir des relations de travail efficaces. Ceci exige que les ISC et leur parlement soient en communication constante et évaluent en continu leurs relations, afin que les travaux de I'ISC fassent l'objet d'un suivi parlementaire efficace.

Au vu des situations très différentes d'un pays à l'autre, il ne peut y avoir un seul modèle de relations de travail efficaces entre les ISC et leur parlement. Ce qui fonctionne dans un pays à un moment donné peut ne pas marcher dans un autre, et ce qui marche dans un pays à un moment donné peut ne pas fonctionner dans ce même pays à un autre moment. Il est impératif d'accepter qu'il n'existe aucune solution idéale pour une relation de travail efficace : c'est un processus qui doit rechercher l'excellence dans une situation donnée, en testant les pratiques pertinentes appliquées dans d'autres pays, et en gardant à l'esprit les objectifs majeurs, à savoir l'impact des travaux de I'ISC et sa contribution à l'ensemble du système de contrôle de la gestion publique. 


\section{ANALYSE DES RELATIONS DE TRAVAIL ENTRE LES ISC ET LES PARLEMENTS : BONNES PRATIQUES EN EUROPE ET FACTEURS-CLÉS POUR UNE RELATION EFFICACE}

\subsection{Introduction}

Le présent chapitre analyse les caractéristiques et les pratiques concernant les relations entre les ISC et leur parlement. Les informations proviennent essentiellement de l'enquête et des matériaux des ISC et des parlements disponibles au public. Pour présenter les pratiques en Europe et mettre en évidence les facteurs-clés pour une relation efficace ainsi que les domaines de bonnes pratiques, le présent chapitre s'appuie sur les cadres / normes décrits aux chapitres 1 et 2 et sur le contexte présenté au chapitre 3.

\subsection{Programme de contrôle}

En sélectionnant les sujets d'audit et en réalisant la planification, la programmation, l'exécution, la communication et le suivi de leurs travaux, les ISC ne doivent faire l'objet d'aucune directive ou ingérence de la part du parlement ou du gouvernement. Mais parallèlement, les ISC peuvent accepter certaines demandes d'enquête ou de contrôle sollicitées par le parlement ou le gouvernement. Elles doivent en outre veiller à intégrer les attentes des parties prenantes dans leurs programmes de contrôle, à travailler avec ces parties prenantes et à tenir compte de leurs points de vue.

En pratique, des dispositifs juridiques et des pratiques variés permettent aux parties prenantes de demander un audit ou de contribuer aux programmes de travail des ISC des Pays membres de I'UE et du Réseau. La figure 4.1 résume ces dispositifs.

Figure 4.1. Dispositifs de coopération sur les sujets d'audit et le programme de travail (PdT)

Consultation sur le PdT (nombre d'ISCs)

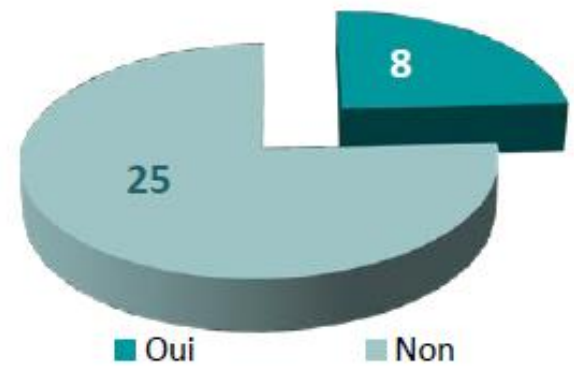

Disposition juridique autorisant une demande d'audit (nombre d'ISCs)

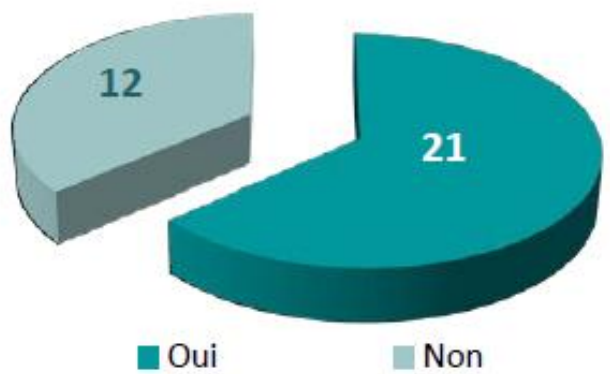

Source : résultats de l'enquête.

Remarques :

ISC avec consultation sur leur proposition de programme de travail : Albanie, Ancienne République yougoslave de Macédoine, Kosovo, Lituanie, Pologne, Slovénie, Suède et Royaume-Uni.

ISC avec dispositions juridiques autorisant une demande d'audit : Autriche, Belgique, Bulgarie, Bosnie-Herzégovine, Croatie, République tchèque, Danemark, Espagne, France, Hongrie, Kosovo, Lituanie, Luxembourg, Malte, Pologne, Portugal, Roumanie, Slovaquie, Slovénie, Turquie et Royaume-Uni. 
Les réponses au questionnaire indiquent que 21 pays ont des dispositions juridiques qui habilitent le parlement à solliciter des audits, bien que la décision finale d'accepter la demande soit souvent du ressort de I'ISC (9 sur 21). Toutefois, dans d'autres cas (12 sur 21), I'ISC est dans l'obligation d'accepter la requête, bien que le nombre d'audit à exécuter sur demande puisse être limité pour préserver la capacité opérationnelle de I'ISC et lui éviter une charge de travail excessive. Par exemple, I'ISC bulgare doit accepter jusqu'à cinq demandes de contrôle par an, I'ISC autrichienne n'a pas à en accepter plus de trois à la fois, et celle du Portugal seulement deux par an. Si d'autres demandes sont formulées, I'ISC portugaise s'efforce de les intégrer à son programme de travail annuel. En Lituanie, I'ISC doit accepter toutes les demandes d'audit mais en pratique, une seule demande est en moyenne formulée par an, ce qui ne pose pas de difficultés à I'ISC. En Slovénie, la décision finale revient à l'ISC, mais le parlement a le droit de soumettre des propositions parmi lesquelles I'ISC doit en retenir au moins cinq, et au moins deux doivent être proposées par des parlementaires de l'opposition. Dans son rapport annuel, I'ISC doit rendre compte de toutes les propositions qui lui ont été adressées ainsi que de celles qu'elle a acceptées. Cette pratique permet à l'ISC de sélectionner les propositions et de décider directement de leur périmètre et de la méthodologie à appliquer.

Dans certains pays, la capacité juridique de solliciter un audit est limitée aux membres de la commission de contrôle. À Malte, trois membres sur les sept que compte la commission de contrôle sont conviés à formuler une demande d'audit. En Irlande, les règles de procédure du parlement stipulent que la commission de contrôle peut proposer un audit, sans préjudice de l'indépendance de I'ISC.

Dans les pays qui n'ont pas de dispositions juridiques (12 ISC), dans neuf cas, le parlement n'est pas consulté sur le programme de travail, mais cela ne signifie pas que ses propositions d'audit sont mises de côté. Par exemple l'ISC lettone s'enquiert activement des propositions une fois par an et en Estonie, le parlement rassemble les propositions à soumettre à l'examen de I'ISC. L'ECA prend également en considération les propositions et demandes du Parlement européen et de ses commissions lorsqu'elle prépare son programme de travail annuel. En Allemagne, toutes les demandes émises par la commission parlementaire spécialisée sont acceptées à moins qu'elles ne causent des préjudices politiques à I'ISC ou qu'elles n'utilisent trop de ressources.

À une exception près, (la Grèce, en raison du statut judiciaire de I'ISC), toutes les ISC sondées prennent en considération les propositions du parlement sur les sujets d'audit possibles ou sur les entités à contrôler. Certaines sollicitent même activement l'avis du parlement, se conformant ainsi aux normes imposant aux ISC de tenir compte des points de vue et des attentes des parties prenantes dans l'élaboration de leur programme d'audits. Cela devrait accroître l'attention que les parlements accordent aux rapports des ISC en général, et contribuer à améliorer leur impact.

Dans le cadre de sa coopération avec le parlement et les parties prenantes, il est important que l'ISC gère les différentes attentes. Entre autres son mandat, ses ressources et son expertise spécifique peuvent être des facteurs limitant sa capacité à répondre aux demandes parlementaires ou à produire des rapports de qualité dans les délais demandés. Le traitement de demandes d'audit motivées par des raisons politiques peut être un défi pour I'ISC, qui pourrait se retrouver dans une tourmente politique susceptible de nuire à son image d'institution professionnelle, objective et indépendante. Par conséquent, il peut être utile de répertorier les instruments d'investigation potentiellement à la disposition du parlement, y compris les audits de I'ISC, et de débattre avec le parlement des circonstances et des conditions qui feraient privilégier un audit ou une enquête de I'ISC. Par ailleurs, I'ISC pourrait définir des critères clairs servant à évaluer les demandes d'audit et les communiquer au parlement pour que ce dernier comprenne sa démarche. 
Mises à part les propositions concrètes sur une thématique d'audit ou une entité à contrôler, les parlements peuvent jouer un rôle également en prodiguant des conseils sur l'adoption de plans de contrôle stratégiques à plus long terme. Par exemple l'ISC de Lettonie a interrogé des parlementaires sur le système budgétaire afin d'identifier les domaines d'intérêt qui pourraient être incorporés dans la planification à long terme des contrôles. Cela montre que, quel que soit le contexte juridique, pratiquement toutes les ISC sollicitent la contribution du parlement pour montrer la pertinence de leur mission aux parties prenantes. Elles doivent s'assurer que leur coopération avec le parlement est efficace, et que l'exécution des audits obligatoires sur demande ne compromet pas leur intégrité et leurs priorités. Le tableau reprend les bonnes pratiques concernant les programmes de travail des ISC, en partant de I'hypothèse que les ISC sont libres de toute directive ou ingérence du parlement ou du gouvernement lorsqu'elles établissent leurs programmes de contrôle.

Tableau 2. Bonne pratiques concernant le programme de travail des ISC

\section{Bonne pratiques destinées au programme de travail}

\begin{tabular}{|l|l|}
\hline Demande d'audit & $\begin{array}{l}\text { Convenir avec le parlement d'une procédure de consultation avant la demande } \\
\text { d'audit officielle. }\end{array}$ \\
\hline $\begin{array}{l}\text { Disposition juridique } \\
\text { autorisant à demander } \\
\text { un audit }\end{array}$ & $\begin{array}{l}\text { Limiter le nombre d'audits à réaliser sur demande, simultanément ou tout au long } \\
\text { de l'année. }\end{array}$ \\
\hline Programme de travail & $\begin{array}{l}\text { Informer le parlement du programme de travail (si la loi le permet). } \\
\text { Donner au parlement l'occasion d'apporter régulièrement une contribution } \\
\text { (réunions, entretiens). } \\
\text { Assurer la liaison entre la commission compétente et les autres commissions } \\
\text { concernées. } \\
\text { Suivre les discussions parlementaires pertinentes afin de définir les audits } \\
\text { potentiels. }\end{array}$ \\
\hline Plan stratégique & $\begin{array}{l}\text { Donner au parlement l'occasion d'apporter une contribution (réunions, entretiens, } \\
\text { conférences). }\end{array}$ \\
\hline
\end{tabular}

Source : résultats de l'enquête.

\section{3 Établissement et soumission des rapports au parlement}

Les résultats de l'enquête confirment que toutes les ISC rendent compte au parlement, le plus souvent sur le fondement d'une obligation constitutionnelle ou d'une autre disposition juridique. Dans certains cas, les rapports d'audit, portant majoritairement sur les états financiers, sont adressés aux entités contrôlées, qui sont à leur tour tenues de les envoyer au parlement, accompagnés du rapport de l'ISC, comme c'est le cas au Royaume-Uni. Dans de nombreux pays, le rapport est publié au moment de sa soumission au parlement, mais certains pays suivent des règles particulières : au Luxembourg par exemple, un rapport ne peut être rendu public qu'après sa soumission au parlement. 
Tandis que toutes les ISC élaborent des rapports à l'intention du parlement, l'enquête montre clairement des différences substantielles d'un pays à l'autre :

Allemagne : L'ISC compile un rapport annuel qui regroupe les constatations d'audit importantes ainsi que les recommandations formulées dans ses audits de l'année. Il s'agit d'un rapport complet qui couvre dans une certaine mesure tous les types d'audit, tout en insistant sur l'audit de performance. En outre, elle peut à tout moment rédiger des rapports spéciaux pour informer les organes législatifs et le gouvernement des questions revêtant une importance particulière; toutefois, le rapport annuel reste l'outil principal par lequel elle rend compte de ses contrôles.

Grèce : I'ISC soumet deux rapports annuels, le Rapport annuel et la Déclaration annuelle. Le Rapport annuel couvre les résultats des opérations et des observations d'audits, il commente les violations détectées des règlements administratifs et financiers ou du budget, et préconise des mesures pour éviter que ces violations ne se reproduisent et pour réformer et améliorer la législation portant sur la compétence de I'ISC. La Déclaration annuelle concerne l'état financier annuel et le bilan de l'État; elle rend compte de l'exécution du budget et de l'exactitude des comptes.

Turquie : L'ISC envoie deux types de rapports au parlement, de caractère général et institutionnel. Elle soumet cinq rapports généraux par an, couvrant des domaines comme l'exécution du budget de l'État, les statistiques financières préparées par le ministère des finances, l'audit des organismes publics et l'audit des entreprises d'État. Les rapports institutionnels, 286 en 2015, rendent compte des différents audits institutionnels réalisés par l'ISC. Ils incluent les audits financiers et de conformité et, dans certains cas, des audits de performance qui examinent les indicateurs de performance transmis. En outre, l'ISC transmet directement aux parlements locaux plusieurs centaines de rapports sur les administrations locales.

Royaume-Uni : les résultats des travaux d'audit financier de I'ISC (incluant l'audit de conformité) sont transmis au parlement sous la forme d'une opinion publiée avec les états financiers annuels de chaque entité contrôlée et, lorsque le président de l'ISC le juge nécessaire, accompagnée d'un rapport connexe. Ces documents sont généralement soumis au parlement par l'entité contrôlée bien que dans quelques cas, I'ISC s'en charge directement, ce qui représente plus de 350 rapports individuels présentés en 20152016. L'ISC soumet tous ses rapports d'audit de performance directement au parlement (60 en 20152016).

Des différences significatives existent dans le nombre de rapports que les ISC soumettent à leur parlement : de 2 en Grèce à 443 en Croatie, comme le montre la figure 4.2. Il convient de noter que le nombre de rapports reflète simplement le mandat, le cadre juridique général et les compétences de I'ISC, et ne donne en aucun cas d'indication sur la qualité des rapports ou du travail de contrôle sous-jacent. 


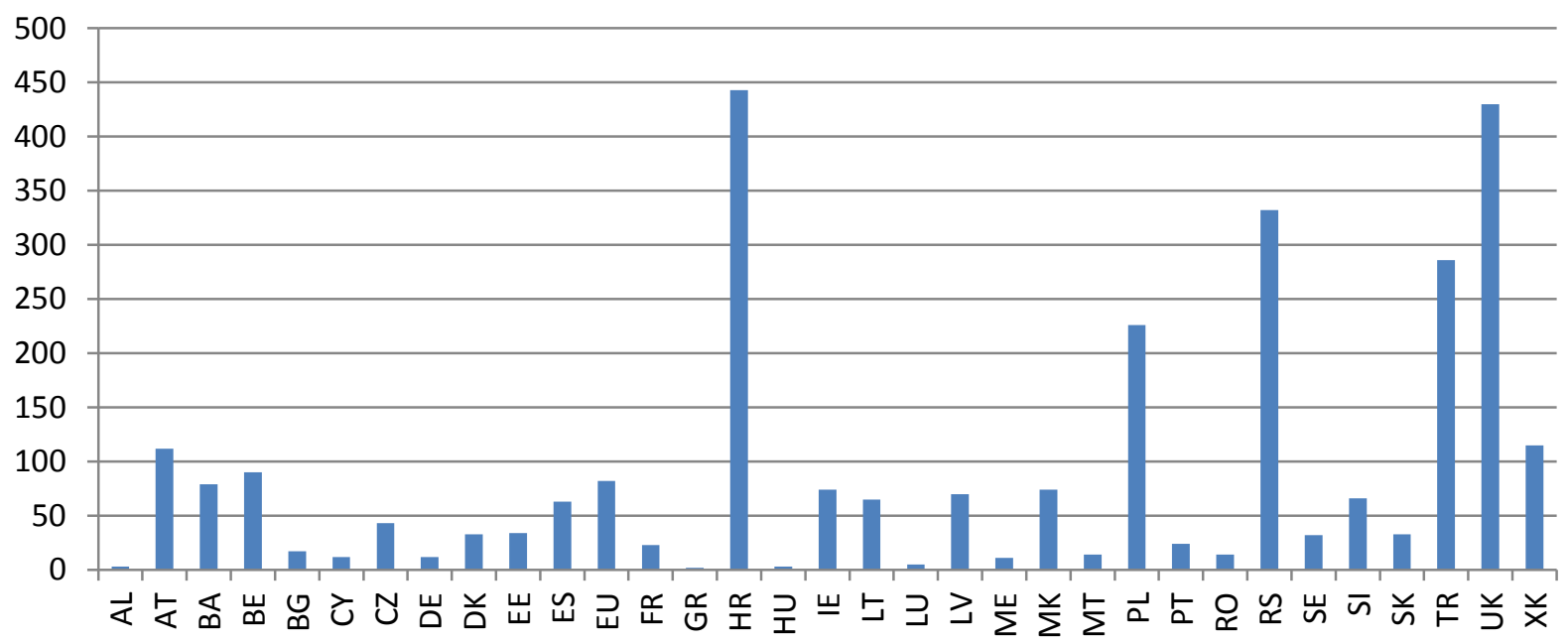

Sources : résultats de l'enquête et sites Internet des ISC.

Remarque : l'année de référence est 2014 pour la Belgique.

Abréviations : AL (Albanie), AT (Autriche), BA (Bosnie-Herzégovine), BE (Belgique), BG (Bulgarie), CY (Chypre), CZ (République tchèque), DE (Allemagne), DK (Danemark), EE (Estonie), ES (Espagne), EU (Union européenne), FR (France), GR (Grèce), HR (Croatie), HU (Hongrie), IE (Irlande), LT (Lituanie), LU (Luxembourg), LV (Lettonie), ME (Monténégro), MK (Ancienne république yougoslave de Macédoine), MT (Malte), PL (Pologne), PT (Portugal), RO (Roumanie), RS (Serbie), SE (Suède), SI (Slovénie), SK (République slovaque), TR (Turquie), UK (Royaume-Uni), XK (Kosovo).

Une gestion efficace des rapports par le parlement sera influencée entre autres par le nombre de rapports soumis. Des obligations juridiques déterminent si tous les rapports d'audit individuels doivent être soumis séparément ou s'ils peuvent être résumés, par exemple dans le rapport annuel de l'ISC. L'ISC du Monténégro par exemple inclut des résumés de tous les rapports dans son rapport annuel, mais elle remet également une sélection de rapports individuels qui doivent être selon elle examinés par le parlement ; cette procédure permet de concentrer l'attention sur les rapports les plus importants.

Une autre option consiste à soumettre tous les rapports, mais en recommandant des examens prioritaires. Au Royaume Uni, la commission de contrôle parlementaire, à savoir la commission des comptes publics, sélectionne quels rapports d'audit de performance elle va examiner en fonction des résumés fournis par I'ISC (approximativement la moitié des rapports soumis) et elle examine les rapports d'audit financier uniquement si I'ISC souligne des sujets spécifiques à débattre. Les ISC disposent par conséquent de plusieurs options qui permettent au parlement de sélectionner des rapports ou des sujets majeurs, l'enjeu commun étant d'instaurer, en concertation avec le parlement, des procédures permettant d'aider ce dernier à traiter plus à fond les rapports qui l'exigent. Un examen approfondi des rapports dépend naturellement de la capacité d'absorption du parlement, et la distinction entre les rapports annuels et les rapports spéciaux doit être également prise en compte.

Il existe différents moyens de soumettre les rapports au parlement. Certaines ISC et certains parlements suivent une procédure plutôt formelle selon laquelle le président de l'ISC envoie le rapport au président du parlement (République tchèque), qui le transmet ensuite à la commission compétente. D'autres ISC envoient directement les rapports à la commission spécialisée (Albanie) ou même à tous les parlementaires (UE). Dans certains cas, le parlement distribue lui-même les rapports (Croatie), mais en général, le rapport annuel est présenté par le président de I'ISC en séance plénière ou devant la commission spécialisée. Dans certaines situations, il est possible de poser des questions à l'ISC pendant 
la présentation, tandis que d'autres ISC rédigent un communiqué sur les rapports d'audit pour fournir des éléments de contexte et des informations plus détaillées. Ces communiqués peuvent aider les parlementaires et leurs organes de travail à traiter les rapports d'audit de manière professionnelle.

Toutes les ISC publient leurs rapports sur leurs sites Internet et un grand nombre rédige des communiqués de presse lors de la publication d'un rapport. Les rapports contiennent souvent un résumé qui en facilite la lecture et oriente les lecteurs vers des sujets d'intérêt spécifiques; par ailleurs, toutes les ISC déclarent que leurs rapports comportent des conclusions et des recommandations claires. Certaines ISC, par exemple I'ECA, ont introduit un nouveau type de rapport établi à partir de l'ensemble des observations contenues dans les rapports précédents qui traitaient d'une thématique particulière. Ceci permet de faire des observations et des conclusions d'un niveau supérieur sur les problématiques systémiques et leurs causes primaires.

En matière de rapports, les résultats indiquent sans ambiguïté qu'il n'y pas de méthode unique pour remplir les exigences énoncées dans les normes ISSAI, mais qu'au contraire une variété de dispositifs sont nécessaires en fonction du contexte de I'ISC; quelques bonnes pratiques sur les rapports sont récapitulées dans le tableau 3. Quelles que soient les moyens utilisés par les ISC pour divulguer leurs travaux, elles doivent coopérer avec le parlement pour veiller à ce qu'il traite leurs rapports avec efficacité. Ceci permet au parlement de se concentrer sur les rapports, les problématiques ou les thématiques fondamentaux présentés par les ISC, qui peuvent ainsi remplir l'objectif des ISSAI, à savoir que le parlement prenne en considération les domaines pertinents et examine les thématiques et les constatations récurrentes.

Tableau 3. Soumission des rapports des ISC au Parlement : bonnes pratiques

\section{Bonnes pratiques concernant l'établissement et la soumission des rapports}

Soumettre au parlement les rapports et les publier simultanément.

S'assurer que les rapports sont distribués aux parlementaires / commissions concernés.

Proposer des présentations et des notes d'information sur les rapports.

Diffuser des communiqués de presse pour mettre en lumière les questions importantes.

Sélectionner les rapports soumis ou faire des suggestions sur les rapports à sélectionner pour examen.

Regrouper dans des rapports thématiques les résultats d'audits précédents.

Source : résultats de l'enquête. 


\subsection{Communication}

Un grand nombre d'ISC interrogées ont pris des initiatives pour répondre aux normes imposant aux ISC d'établir de bonnes relations de travail avec le parlement, de sensibiliser ce dernier au rôle qu'elles jouent et de l'aider à comprendre les rapports d'audit. L'ISC grecque, nonobstant son statut judiciaire, a organisé des présentations d'information sur les rapports à l'intention de deux commissions parlementaires car elle estime que l'institutionnalisation de ce type d'activités est positive pour ses relations avec le parlement. L'ISC turque a mis en œuvre une nouvelle stratégie de communication à l'intention du parlement et d'autres organes, et I'ISC belge emploie des experts en communication qui aident les contrôleurs à rédiger des rapports clairs et compréhensibles. L'ISC lettonne communique au parlement son calendrier d'envoi des rapports pour attirer le maximum d'attention sur ses rapports d'audit, tandis que les ISC albanaise, serbe, hongroise et lituanienne organisent des conférences et des tables rondes sur des sujets spécifiques auxquelles sont invités les parlementaires, ou bien elles offrent des ateliers communs.

Ces activités suscitent une meilleure compréhension et favorisent un accord sur les procédures de coopération. Celles-ci prennent parfois la forme d'un protocole d'accord, comme dans l'Ancienne République yougoslave de Macédoine, en Hongrie et en Serbie. L'ISC de I'Ancienne République yougoslave de Macédoine a mis au point du matériel d'orientation et un livret à l'intention des parlementaires, qui leur présentent le rôle et le travail de I'ISC et expliquent comment lire les rapports d'audit. L'ISC suédoise, quant à elle, organise après chaque élection un atelier destiné aux parlementaires sur le rôle et les activités de I'ISC. Toutes les ISC entretiennent, sous une forme ou une autre, des échanges avec le parlement, allant d'un échange relativement formel une fois par an à des contacts informels presque quotidiens. La compréhension mutuelle est améliorée grâce à des réunions, mais également par des entretiens avec les parlementaires, comme le font les ISC lettonne et serbe. Pour susciter son attention, I'ISC hongroise réalise des études sur des sujets qui intéressent le parlement.

L'enquête met également en lumière le fait que la communication des ISC s'efforce d'attirer l'attention du parlement sur leurs rapports en s'adressant aux commissions parlementaires (Albanie, ECA, République tchèque, Royaume-Uni), ou en encourageant simplement l'établissement d'une commission ou sous-commission de contrôle (Ancienne République yougoslave de Macédoine, Croatie, Roumanie, Turquie). Une autre méthode utilisée pour améliorer la compréhension mutuelle et pour établir de bonnes relations de travail est le détachement de personnel de l'ISC auprès du parlement, ou même la mise en place de programme de détachements croisés. L'Irlande, la Suède et le Royaume-Uni en sont des exemples, et l'ISC du Kosovo est en train d'étudier cette possibilité ; le tableau 4 reprend quelques exemples de bonnes pratiques.

La communication est à double sens : l'efficacité de la politique et des activités de communication d'une ISC dépend de la manière dont elles sont perçues et reçues par le parlement, la dynamique de l'environnement politique étant particulièrement importante. Par conséquent, il est important que l'ISC actualise continuellement sa politique de communication, qu'elle soit sensibilisée aux nouvelles opportunités et qu'elle essaie de nouvelles méthodes, dont celles issues des développements techniques. 
Tableau 4. Bonnes pratiques destinées aux ISC dans leur communication avec le parlement

\section{Bonnes pratiques destinées aux ISC dans leur communication avec le parlement}

Organiser régulièrement des réunions avec le parlement, complétées par des échanges informels au niveau opérationnel.

Coordonner les ordres du jour et les calendriers de soumission des rapports avec le parlement.

Organiser des réunions de travail, des tables rondes et des ateliers.

Publier du matériel et des livrets d'orientation.

Dispenser des programmes de formation/d'orientation sur I'ISC à l'intention des nouveaux parlementaires.

Établir avec le parlement un protocole d'accord définissant les procédures de coopération.

Élaborer une politique de communication.

Améliorer la compréhension mutuelle par le détachement de personnel.

Source : résultats de l'enquête.

\subsection{Suivi des observations précédentes et des recommandations de l'ISC}

Le suivi des observations et des recommandations de I'ISC peut être divisé en trois catégories. En ce qui concerne les ISC qui ont une fonction juridictionnelle, la suite apportée aux jugements est mise en application par les tribunaux judiciaires ou disciplinaires compétents; I'ISC ou le parlement n'intervient plus. La seconde catégorie concerne les constatations de fraude ou de corruption présumée. Dans ce cas, I'ISC doit saisir le bureau du procureur qui poursuivra l'enquête ; de même, le rôle de I'ISC ou du parlement s'arrête là, bien qu'une information sur l'état d'avancement des dossiers transmis au bureau du procureur soit la bienvenue.

La troisième catégorie regroupe les observations et les recommandations d'audit faites lors des contrôles. Les résultats de l'enquête démontrent clairement que toutes les ISC surveillent la suite apportée à leurs observations et l'application des recommandations formulées dans leurs rapports, conformément aux attentes énoncées dans les normes. De nombreuses ISC tiennent à jour une base de données : La Bosnie-Herzégovine et le Monténégro se servent d'un fichier Excel, la Bulgarie et l'Irlande utilisent un registre électronique et I'Ancienne République yougoslave de Macédoine un logiciel spécialisé. Un grand nombre d'ISC gère cette base de données en interne, mais certaines rendent les informations publiques. En Lituanie par exemple, le statut de la mise en œuvre des recommandations est publié en temps réel sur le site Internet de I'ISC, et la Serbie a l'intention de faire la même chose ; I'ISC bulgare publie les affaires dans lesquelles un organisme contrôlé n'a pas mis en œuvre les recommandations dans les temps impartis. L'ISC suédoise communique annuellement au parlement sur le suivi des observations et des recommandations figurant dans ses rapports d'audit ; I'ISC lituanienne fait de même deux fois par an. Certaines ISC distinguent dans leurs rapports les recommandations acceptées et les recommandations refusées par l'entité contrôlée. Au Danemark et en Lettonie, les ISC 
travaillent étroitement avec leur commission parlementaire respective pour piloter le suivi des rapports et exiger des mesures lorsque la réponse de l'entité contrôlée ne donne pas satisfaction.

II est courant que les ISC intègrent leurs activités de suivi dans un mécanisme qui examine périodiquement la mise en œuvre des recommandations, la communication du suivi et la programmation des audits de suivi. À titre d'exemple, I'ISC de Bosnie-Herzégovine inclut systématiquement dans ses rapports la mise en œuvre des recommandations faites lors de contrôles précédents, et le système de suivi de I'ISC autrichienne est intégré dans son cycle de contrôles annuel. Dans l'année qui suit l'audit, les entités contrôlées doivent fournir des informations sur la mise en œuvre des recommandations : les résultats sont publiés dans le rapport d'activité annuel de I'ISC et utilisés pour sélectionner les sujets susceptibles de faire l'objet d'audits de suivi ciblés portant sur la mise en œuvre des recommandations.

Cette procédure est relativement facile à appliquer aux audits de conformité et / ou financiers mais elle est plus compliquée dans le cas des audits de performance, dans la mesure où le suivi de ces audits fait normalement partie du rapport annuel. Par exemple, dans son rapport annuel sur les comptes de l'État, I'ISC portugaise rend compte des recommandations acceptées dans le rapport précédent et de leur mise en œuvre. De même, dans son rapport d'activité, l'ECA communique le pourcentage des recommandations mises en œuvre par la $\mathrm{CE}$, mais elle a également introduit des rapports consolidés qui traitent spécifiquement du suivi de ses recommandations, et elle reprend systématiquement ses recommandations dans son rapport annuel. En ce qui concerne les rapports consolidés portant sur le suivi des rapports d'audit de performance précédents, I'ECA prend un échantillon de toutes les recommandations faites dans plusieurs rapports et elle analyse leur degré de mise en œuvre. L'ISC suédoise a la même approche, et soumet un rapport annuel qui porte sur les actions prises suite à plusieurs rapports d'audits.

Certaines ISC sont habilitées à enjoindre à l'entité contrôlée de prendre des mesures, en réponse aux recommandations d'audit (en Slovénie par exemple). Dans d'autres pays, les ISC exigent de l'entité contrôlée un plan d'action ou un rapport sur la manière dont cette même entité a réagi aux recommandations d'audit. Chypre a une disposition intéressante, selon laquelle une entité contrôlée doit fournir une déclaration de conformité sur la mise en œuvre des recommandations de l'ISC ; elle doit soumettre cette déclaration avec son projet de budget à l'adoption par le parlement, associant ainsi directement la mise en œuvre des recommandations de l'ISC avec l'approbation des crédits. En Hongrie, I'ISC peut décider d'engager une action disciplinaire à l'encontre du chef de l'entité contrôlée lorsque ses recommandations n'ont pas été prises en compte, et elle peut conseiller de réduire le budget de cette entité. L'ISC polonaise a le droit de proposer des amendements aux lois existantes pour remédier aux manquements relevés pendant les audits (propositions de lege ferenda). Au Monténégro, l'entité contrôlée est tenue par la loi de soumettre un rapport sur l'application des recommandations de I'ISC dans les délais qu'elle a fixés. Des obligations similaires existent dans I'Ancienne République yougoslave de Macédoine et en République slovaque. Le tableau 5 présente quelques exemples de bonnes pratiques relatives aux procédures de suivi des ISC. 
Tableau 5. Bonnes pratiques concernant les procédures de suivi des ISC

\section{Exemples de bonnes pratiques concernant les procédures de suivi des ISC}

Les recommandations sont solidement étayées, pratiques, réalistes et concrètes.

Création d'un mécanisme de pilotage.

Publication de l'état de mise en œuvre des recommandations.

L'état de la mise en œuvre des recommandations est communiqué à intervalles fixes.

Le pilotage est intégré au système de planification des audits de vérification et de suivi.

Une attention particulière est accordée à la communication portant sur la mise en œuvre des recommandations formulées

dans les rapports d'audit de performance.

Un grand nombre d'ISC voit des possibilités d'amélioration dans la procédure de suivi par le parlement : selon l'étude, 11 ISC (33\%) émettent un jugement critique quant au niveau d'efficacité du suivi réalisé par le parlement sur les observations et les recommandations de I'ISC. Et il est signalé que certains parlements n'ont aucune procédure de suivi.

Figure 4.3 Procédures de suivi appliquées par les ISC et les parlements

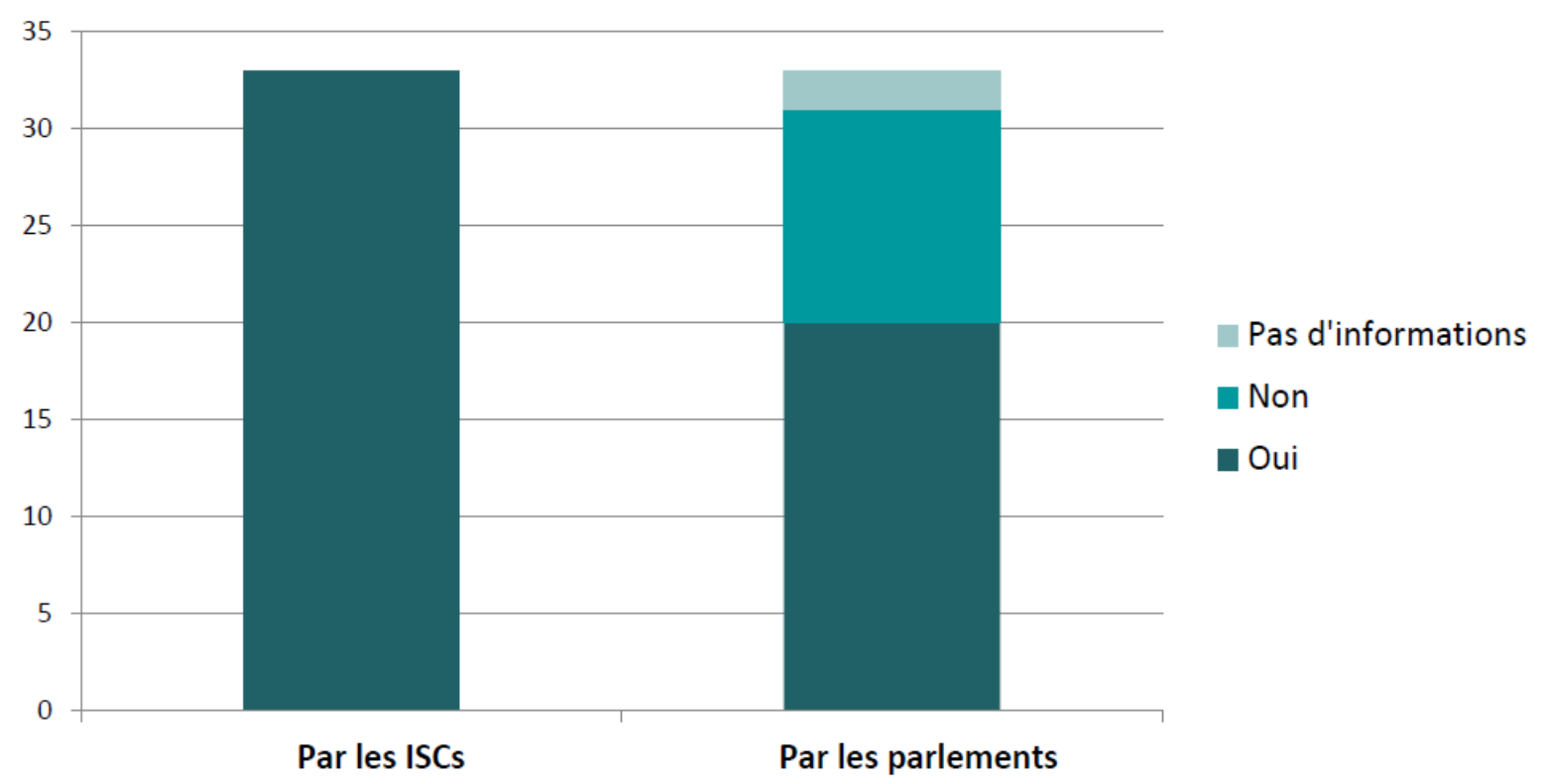

Source : résultats de l'enquête.

Au Danemark, en Lettonie et au Royaume-Uni, I'ISC et la commission parlementaire spécialisée travaillent étroitement lorsqu'il s'agit de surveiller la procédure de suivi et d'exiger des organismes contrôlés qu'ils prennent des mesures. Au Danemark, la commission de contrôle demande aux ministres compétents des déclarations sur les rapports de I'ISC, puis elle demande à I'ISC de commenter ces déclarations. Dans le rapport qu'elle remet au parlement, la commission de contrôle prend en compte la 
réponse du ministre et les remarques de I'ISC pour proposer sa propre conclusion ; cette procédure est illustrée à la figure 4.4 .

Figure 4.4 Procédures de suivi appliquées par l'ISC et le parlement danois

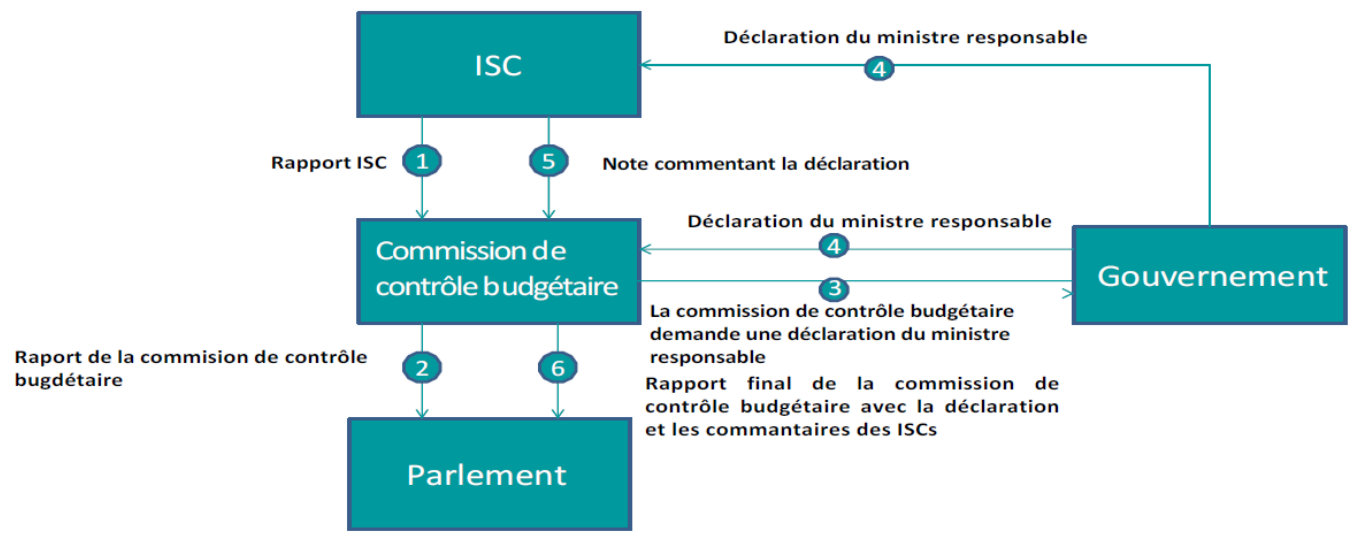

Source : réponses de l'ISC danoise à l'enquête.

Une procédure semblable est appliquée en Lettonie, selon laquelle l'entité contrôlée, lors d'une réunion de la commission de contrôle, lui remet ses commentaires sur le rapport de I'ISC et sur ses recommandations. L'ISC fournit ensuite des commentaires additionnels ou des clarifications. Après délibération des membres de la commission de contrôle, il est établi un calendrier que l'entité contrôlée doit respecter pour rendre compte de la mise en œuvre des recommandations ; I'ISC est conviée à émettre un avis sur l'avancement de leur mise en œuvre. Les ISC de Croatie, de l'ancienne République yougoslave de Macédoine, de Roumanie, de la République slovaque et de la Turquie estiment que la création d'une commission de contrôle parlementaire spécialisée serait bénéfique à un traitement efficace des rapports des ISC, y compris celui du suivi des observations et des recommandations. En tenant compte des expériences menées par les pays qui ont déjà une commission de contrôle de cette nature (voir la section 4.7), cette position paraît fondée, notamment concernant les rapports d'audit de conformité et financiers. L'ISC estonienne pense toutefois qu'elle peut avoir davantage d'influence en établissant des liens avec le gouvernement plutôt qu'avec le parlement mais, bien que cela s'avère vérifié dans certaines circonstances, les ISC ont également pour mission de contribuer à l'amélioration du système de contrôle de la gestion publique, ce qui exige l'engagement du parlement.

Les ISC qui sont généralement satisfaites de la manière dont le parlement traite leurs rapports d'audit perçoivent encore des possibilités d'amélioration : L'ISC de Bosnie-Herzégovine par exemple, est satisfaite du traitement de ses rapports d'audit de conformité et financier par le parlement, fondé sur des procédures bien formalisées au sein du parlement, mais elle souhaiterait avoir des procédures semblables pour les rapports d'audit de performance. 
Tableau 6. Bonnes pratiques dans les procédures de suivi parlementaires

Exemples de bonnes pratiques concernant les procédures de suivi parlementaires

Mise en place de procédures normalisées destinées au traitement des rapports de l'ISC.

Organisation d'auditions en présence des entités contrôlées.

Demande d'un plan d'action au gouvernement ou à l'entité contrôlée.

Des dates butoirs sont associées aux plans d'action ou aux mesures à prendre.

Des sanctions (politiques, financières et disciplinaires) sont considérées par I'ISC ou le parlement en cas de non-respect grave des recommandations.

Obligation des entités contrôlées ou du gouvernement de fournir des rapports de mise en œuvre.

Source : résultats de l'enquête.

\subsection{Rapports concernant la performance de I'ISC}

Selon I'enquête, toutes les ISC, à l'exception de Chypre et de l'Irlande, informent le parlement de leurs activités de l'année antérieure, soit sous la forme d'un rapport d'activité annuel individuel (29 ISC) ou d'un chapitre spécial inclus dans le rapport annuel (Grèce, Monténégro). L'ISC irlandaise publie toutefois un rapport d'activité annuel sur son site Internet. Cela montre que toutes les ISC interrogées prennent au sérieux l'obligation de rendre compte de leur propre performance.

En règle générale, ces rapports d'activité englobent un compte-rendu sur la manière dont les ressources ont été utilisées. L'ISC du Royaume-Uni publie une estimation des économies faites dans le secteur public sur la base de ses rapports et recommandations. C'est un exercice compliqué, mais l'accord de I'entité contrôlée figure dans la méthodologie, ce qui accroît la fiabilité des résultats. Pour 2015, I'ISC du Royaume-Uni a calculé que pour chaque livre GBP de son propre budget, elle a obtenu la réalisation de 19 livres GBP d'économies. C'est une mesure de l'impact et de la performance, qui facilite également la fixation d'objectifs quantitatifs pour la prochaine période de planification.

Outre le rapport annuel sur leurs activités et leurs ressources, les ISC peuvent également témoigner de leur souci de rendre compte en invitant des vérificateurs externes à réaliser des évaluations indépendantes effectuées par des pairs, et en rendant public leurs rapports. Selon l'enquête menée par la sous-commission INTOSAI sur les évaluations réalisées par les pairs en 2015, 20 ISC sur 36 de I'Union européenne et des pays du Réseau ont été soumises à une évaluation par les pairs entre 1999 et 2015, certaines à plusieurs reprises pendant cette période ; étant donné le taux de réponse à l'enquête (globalement $41 \%$ ), le nombre véritable d'évaluations par les pairs est probablement sous-estimé. 15 ISC de I'UE et du Réseau ont publié leurs rapports d'évaluation par les pairs sur le site Internet de la Commission INTOSAI chargée du renforcement des capacités.

Les rapports d'activité annuels, l'examen externe des comptes de l'ISC par des contrôleurs externes et les évaluations périodiques par les pairs, toutes ces activités contribuent à donner à l'ISC I'image d'une institution professionnelle, objective, transparente et efficace. Cela aide à renforcer la confiance et l'autorité accordées au travail de I'ISC, et donc à créer ou renforcer son influence. Le tableau 7 présente quelques exemples de bonnes pratiques. 
Tableau 7. Bonnes pratiques relatives à la communication de la performance des ISC

\section{Exemples de bonnes pratiques concernant la communication sur la performance des ISC}

Publication de rapports annuels certifiés portant sur les comptes et l'utilisation des ressources.

Rédaction et publication de rapports annuels sur les activités et la mise en œuvre du programme de travail.

Réalisation périodique d'évaluations externes par les pairs, dont les rapports sont mis à disposition du public.

Estimation des économies obtenues dans le secteur public.

Source : résultats de l'enquête.

\subsection{Traitement des rapports des ISC par le parlement}

La section 3.3 a résumé les principaux dispositifs parlementaires destinés à examiner les travaux des ISC ; par ailleurs, elle a présenté les caractéristiques distinctives des commissions budgétaires, des commissions et sous-commissions de contrôle qui sont les solutions organisationnelles les plus courantes pour traiter les rapports des ISC. Dans un nombre croissant de pays, les commissions thématiques et sectorielles prennent également de l'importance, encouragées par I'ISC ou par la commission de contrôle elle-même.

Aux Pays-Bas par exemple, la commission de contrôle parlementaire joue un rôle de coordination afin d'encourager une implication des commissions sectorielles. Cependant, sans garde-fous procéduraux, ceci risque de faire émerger une compétition entre les commissions. Les ISC d'Albanie et du RoyaumeUni, ainsi que l'ECA, s'efforcent d'impliquer davantage les commissions sectorielles dans les débats sur les rapports des ISC, par exemple en offrant des notes d'information sur les rapports spéciaux. L'ISC de la République tchèque a fait le constat qu'un intérêt accru pour ses rapports de la part des commissions sectorielles serait bénéfique, et en Turquie, le parlement a une commission sectorielle chargée d'examiner les rapports d'audit sur les entreprises publiques, tous les autres rapports d'audit étant traités par la commission budgétaire.

La figure 4.5 reprend les principales commissions qui traitent les rapports des ISC dans les Pays membres de l'Union européenne et dans les pays du Réseau. 


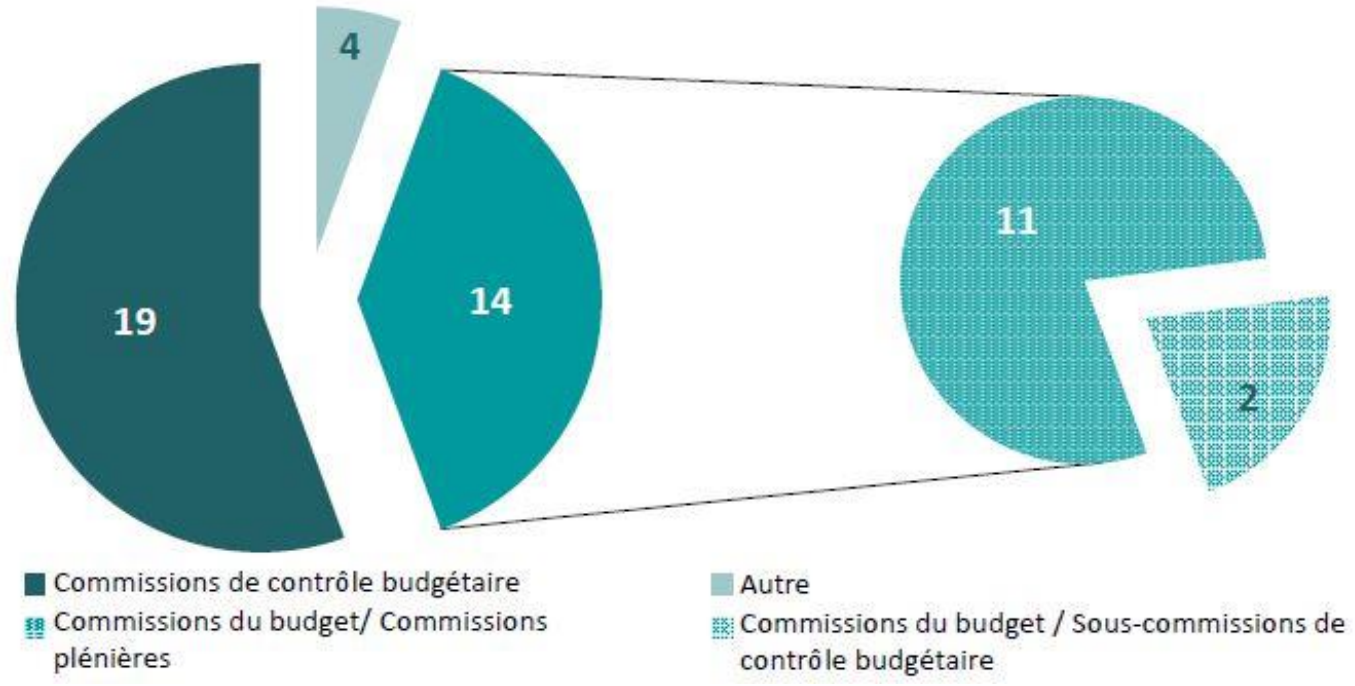

Source : Parlement européen (2012), Résultats de l'enquête et contrôle parlementaire concernant l'exécution du budget, http://www.europarl.europa.eu/RegData/etudes/etudes/join/2012/490661/IPOL-JOIN_ET(2012)490661_EN.pdf.

Remarques :

Rapports des ISC traités par les commissions de contrôle parlementaire : Autriche, Chypre, République tchèque, Danemark, Estonie, Union européenne, Finlande, Allemagne, Grèce, Irlande, Kosovo, Lettonie, Lituanie, Luxembourg, Malte, Pays-Bas, Slovénie, Espagne, Royaume-Uni.

Rapports des ISC traités par les commissions budgétaires : Albanie, Bosnie-Herzégovine, Croatie, France, Ancienne République yougoslave de Macédoine, Hongrie, Italie, Monténégro, Portugal, Roumanie, Turquie.

Rapports des ISC traités par les sous-commissions de contrôle de la commission budgétaire : Bulgarie, Serbie.

La Belgique, la Pologne, la République slovaque et la Suède ont d'autres dispositifs.

Selon l'étude, les ISC de Croatie, de l'Ancienne République yougoslave de Macédoine, de Roumanie et de Turquie encouragent activement la création d'une commission ou d'une sous-commission de contrôle indépendante.

Dans de nombreux pays, outre les discussions au sein de la commission, les débats qui portent sur les travaux de I'ISC ont lieu en séance plénière. Ces débats portent habituellement sur l'exécution du budget de l'État et ils sont souvent liés aux décisions parlementaires officielles sur la clôture des comptes ou sur l'octroi de la décharge budgétaire au gouvernement. Les discussions au sein de la commission servent généralement à préparer les débats qui ont lieu en séance plénière, qui peuvent alors se concentrer plus précisément sur les questions principales et les conclusions à formuler.

Formaliser les procédures de décharge peut s'avérer bénéfique pour la responsabilisation des gestionnaires publics en conférant une plus grande importance politique à la décharge, et en offrant au parlement un cadre normatif pour imposer au gouvernement de rendre compte. En définitive toutefois, la décision de clôturer les comptes ou d'octroyer la décharge est une décision politique, et donc la façon dont le parlement pourra utiliser la décharge dépend de ses éventuelles conséquences; par exemple, le Parlement européen peut reporter l'octroi de la décharge pour faire pression sur la Commission européenne afin que cette dernière corrige ou palie les erreurs ou les disfonctionnements systémiques. Si le Parlement européen refuse la décharge, cela aura comme conséquence politique d'obliger la 
Commission à quitter ses fonctions, processus appelé parfois " l'option nucléaire ». Dans certains pays, les conséquences liées au refus de la décharge sont plus limitées, par exemple en contraignant uniquement le chef de l'entité contrôlée à démissionner. En Bosnie-Herzégovine, où la décharge est liée à l'approbation des budgets individuels des institutions budgétaires pour l'année à venir, le parlement peut imposer une sanction en réduisant le budget si l'ISC émet une opinion défavorable sur les comptes. La figure 4.6 résume les procédures de décharge.

Figure 4.6 Nombre de parlements avec des procédures de décharge effectivement suivies



Procédures de décharge effectivement suivies

Aucune procédure de décharge

Source : résultats de l'enquête.

Remarques :

Procédures de décharge effectivement suivies : Albanie, Belgique, Bosnie-Herzégovine, Chypre, Danemark, Union européenne, Estonie, France, Allemagne, Grèce, Hongrie, Irlande, Lettonie, Lituanie, Monténégro, Pologne, Portugal, Roumanie, Slovénie, Turquie, Royaume-Uni.

Aucune procédure de décharge: Autriche, Bulgarie, Ancienne République yougoslave de Macédoine, Kosovo, Luxembourg, Malte, Serbie (mais possible juridiquement), République slovaque, Espagne, Suède.

Aucune information concernant la Croatie et la République tchèque.

Bien qu'il puisse y avoir une différence entre la décharge et la clôture des comptes, la figure 4.6 englobe les deux.

La décharge est naturellement la dernière phase du cycle budgétaire, et les résultats de la procédure de décharge devraient idéalement être pris en compte dans l'adoption du budget de l'année suivante.

Un grand nombre de parlements invitent à leurs auditions les dirigeants ou les ministres responsables des organisations contrôlées, souvent avec des représentants de I'ISC. Permettre aux entités contrôlées d'expliquer leur position et donner à I'ISC I'opportunité de souligner les problématiques essentielles et de réagir aux arguments de l'entité contrôlée aidera les parlementaires à prendre des décisions éclairées. II convient toutefois de se rappeler que ces auditions consomment des ressources organisationnelles et que par conséquent, la sélectivité est une caractéristique essentielle. La figure 4.7 illustre la part des pays qui offrent un tel dispositif. 
Figure 4.7 Nombre de parlements avec un dispositif permettant de tenir des auditions en présence des entités contrôlées

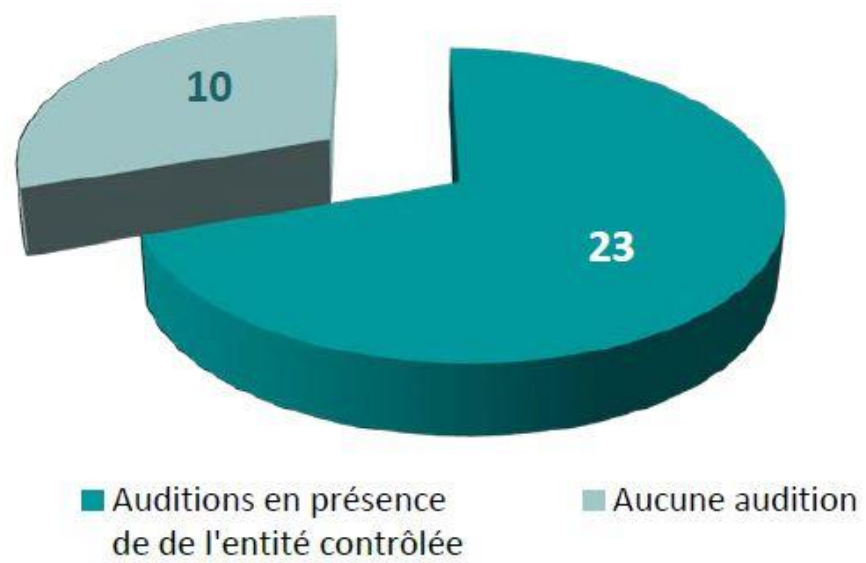

Source : résultats de l'enquête.

Remarques :

Auditions en présence de l'entité contrôlée : Belgique, Bulgarie, Bosnie-Herzégovine, Croatie (occasionnellement uniquement), Chypre, République tchèque, Estonie, Union européenne, France, Allemagne, Irlande, Kosovo, Lettonie, Lituanie, Luxembourg, Malte, Monténégro, Pologne, République slovaque, Slovénie, Suède (occasionnellement uniquement), Turquie, Royaume-Uni.

Aucune audition : Albanie (possible juridiquement), Autriche, Danemark, Ancienne République yougoslave de Macédoine, Grèce, Hongrie, Portugal, Roumanie, Serbie, Espagne.

Dans un nombre limité de parlements européens, il existe un système de préparation des réunions qui consiste à faire appel à des rapporteurs, selon lequel un membre-rapporteur individuel est chargé de préparer une réunion de commission sur un rapport spécifique de l'ISC. Ce membre-rapporteur analyse le rapport, souvent avec l'aide directe de l'ISC : en République tchèque par exemple, le rapporteur peut demander au membre de I'ISC responsable du rapport de l'aider à préparer la discussion parlementaire sur ce rapport. Au Parlement européen, le rapporteur consulte le membre de l'ECA concerné et prépare un rapport en vue de la réunion de la commission au cours de laquelle il est prévu de discuter le rapport de l'ISC. 
Figure 4.8 Nombre de parlements avec un dispositif de désignation d'un rapporteur

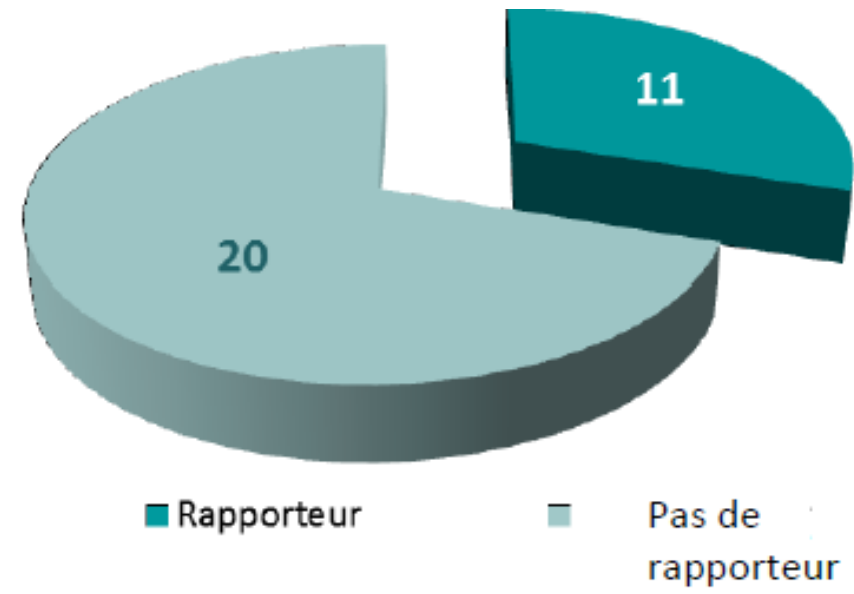

Source : résultats de l'enquête.

Remarques :

Dispositif de désignation d'un rapporteur: Autriche, Belgique, Bosnie-Herzégovine, République tchèque, Union européenne, France, Allemagne, Luxembourg, Pologne, Portugal, Royaume-Uni.

Aucun rapporteur: Albanie (possible juridiquement), Bulgarie, Croatie, Danemark, Ancienne République yougoslave de Macédoine, Grèce, Hongrie, Irlande, Kosovo, Lettonie, Lituanie, Malte, Monténégro, Roumanie, Serbie, République slovaque, Slovénie, Espagne, Turquie.

Aucune information sur Chypre et la Suède.

Vu la grande variété des contextes juridiques et historiques des parlements, il est difficile de récapituler les facteurs de réussite qui permettent d'avoir des relations de travail efficaces avec les ISC ou de suggérer ce qui pourrait être considéré comme de bonnes pratiques. Ce qui marche bien dans un contexte particulier peut ne pas marcher, ou être même irréalisable, dans un autre. Toutefois, les réponses à l'étude fournissent un certain nombre de bonnes pratiques qui valent la peine d'être étudiées (tableau 8).

Tableau 8. Exemples de bonnes pratiques relatives aux dispositifs parlementaires

Bonnes pratiques concernant les dispositifs parlementaires

Les responsabilités de la commission chargée de l'examen des rapports de l'ISC sont organisées de manière adéquate.

Création d'une commission ou sous-commission de contrôle spécialisée.

Des commissions sectorielles participent au traitement des rapports d'audit de performance.

Une procédure de décharge formalisée fait partie du cycle budgétaire dans le cadre de la supervi budgétaire.

Nomination d'un rapporteur pour certains rapports de I'ISC. 
Tenue d'auditions, en présence de l'entité contrôlée.

Fixation d'un calendrier des débats parlementaires portant sur les rapports de I'ISC pour

pouvoir rendre des conclusions en temps opportun.

Mise à la disposition du parlement d'un personnel et de capacités d'analyse suffisantes.

Source : résultats de l'enquête.

\subsection{Observations finales}

L'enquête témoigne d'une grande diversité dans les relations entre les ISC et le parlement. La majorité des ISC réfléchissent en permanence sur les moyens d'améliorer ces relations pour renforcer leur influence, et elles mettent en place des initiatives pour faire évoluer la situation. Bien que les parlements n'aient pas été conviés à participer à l'étude, il est connu que certains parlements ont récemment modifié leurs procédures afin d'utiliser plus efficacement les rapports des ISC, par exemple en instaurant une commission ou sous-commission spéciale, comme en Serbie en 2015. L'ISC serbe avait vivement encouragé cette réforme pendant plusieurs années.

Ceci s'inscrit bien dans le paysage d'ensemble des ISC, qui s'efforcent activement de trouver des opportunités pour structurer et restructurer leurs relations avec le parlement afin de consolider leur influence. L'environnement dynamique, avec ses améliorations dans la qualité de la gestion des finances publiques et, par voie de conséquence l'attention plus soutenue accordée par les ISC à l'audit de performance, laisse à penser que ce processus va se poursuivre. Les bonnes pratiques regroupées dans le présent document peuvent aider les ISC - ainsi que les parlements - à trouver des moyens concrets de renforcer l'efficacité de leurs relations de travail. Le récapitulatif de ces bonnes pratiques, en lien avec les normes et attentes concernées, est présenté à l'Annexe 2, qui peut servir de boîte à outils pour aider les ISC et le parlement à trouver des instruments ou des procédures novateurs. II convient de réaffirmer que, bien que les ISC constituent le principal groupe-cible du présent document, les parlements ont également la responsabilité d'analyser dans quelle mesure leurs relations avec les ISC sont efficaces et de déterminer s'il existe des opportunités de consolider ces relations.

Le rôle que peuvent jouer les medias dans la consolidation de l'influence des ISC et de l'attention parlementaire envers les rapports d'audit était en dehors du champ du présent document, mais I'ISSAI 12, Principe 6-4, déclare que les ISC devront dialoguer de manière adaptée avec les medias en vue de faciliter la communication avec les citoyens. Le triangle des responsabilités de la section 1.1 ne fait pas état des citoyens, mais le parlement représente naturellement les citoyens ; dans ce sens, le dialogue approprié avec les medias contribue au bon fonctionnement du système de contrôle de la gestion publique. Les ISC doivent par conséquent réfléchir dans quelle mesure leur communication avec les medias peut optimiser l'efficacité de leurs relations de travail avec le parlement. 


\section{ANNEXE 1. NORMES INTERNATIONALES ET PRINCIPES RECONNUS PORTANT SUR LES RELATIONS ENTRE LES PARLEMENTS ET LES ISC}

\section{Principes de l'administration publique des pays candidats et candidats potentiels à l'UE}

Mis au point par SIGMA en partenariat avec la Commission européenne en 2014, ces Principes énoncent ce qu'est dans la pratique une bonne gouvernance et soulignent les principales conditions que doivent respecter les pays pendant la procédure d'adhésion à I'UE ; ils sont donc utilisés comme référentiels par les pays candidats et candidats potentiels à I'UE lorsqu'ils souhaitent mesurer la performance de leur administration publique. Dans le domaine de la gestion des finances publiques, les Principes 15 et 16 font référence au rôle de l'ISC :

- Principe 15 : l'indépendance, le mandat et l'organisation de l'institution supérieure de contrôle sont établis et protégés par le cadre constitutionnel et juridique, et ils sont respectés de facto.

- Principe 16 : I'institution supérieure de contrôle se conforme à des normes en restant neutre et objective afin de garantir des audits de très grande qualité qui ont une influence positive sur le fonctionnement du secteur public.

Le Principe 16 comporte deux sous-principes relatifs à la relation de l'ISC avec le parlement : le parlement dispos d'un mécanisme formalisé lorsqu'il examine les rapports de I'ISC, et I'ISC fournit au législateur, et en particulier aux commissions parlementaires, des informations pertinentes et objectives en temps voulu. L'annexe méthodologique comporte un critère supplémentaire, à savoir que le parlement doit prendre dûment en considération les rapports en mettant le gouvernement devant son obligation de rendre compte.

\section{Normes internationales des Institutions supérieures de contrôle (ISSAI)}

ISSAI 1 - La Déclaration de Lima, Section 16. Rapports à l'intention du Parlement et du public :

«La Constitution doit autoriser et obliger l'Institution supérieure de contrôle des finances publiques à présenter, chaque année et de manière autonome, un rapport sur les résultats de son activité au Parlement ou à tout organe étatique responsable; ce rapport doit être publié. Cette mesure permettra une large diffusion de l'information, un examen critique poussé du contenu du rapport et favorisera la réalisation des constatations de l'Institution supérieure de contrôle des finances publiques. »

ISSAI 10 - La Déclaration de Mexico. Existence de mécanismes efficaces de suivi des recommandations des ISC :

"Les Institutions supérieures de contrôle des finances publiques soumettent leur rapports de contrôle au Parlement (ou à une de ses commissions) ou au conseil d'administration de l'entité contrôlée, selon le cas, pour qu'il les examine et qu'il effectue un suivi des recommandations exigeant des mesures correctives.

Les Institutions supérieures de contrôle des finances publiques possèdent leur propre système interne de suivi pour veiller à ce que les entités contrôlées donnent suite de façon satisfaisante à 
leurs observations et à leurs recommandations ou à celles du Parlement (ou d'une de ses commissions) ou de leur conseil d'administration, selon le cas.

Les Institutions supérieures de contrôle des finances publiques soumettent leur rapports de contrôle au Parlement (ou à une de ses commissions) ou au conseil d'administration de l'entité contrôlée, selon le cas, afin qu'il les examine et prenne des mesures, et ce, même lorsqu'elles ont, en vertu de la loi, le pouvoir d'effectuer des suivis et de prendre des sanctions. »

ISSAI 12 - La valeur et les avantages des Institutions supérieures de contrôle des finances publiques - faire une différence dans la vie des citoyens. En 2013, I'INTOSAI a adopté I'ISSAI 12, ensemble fondamental et ambitieux de normes s'appuyant sur des normes INTOSAI existantes. L'ISSAI 12 présente 12 principes axés sur les valeurs et les avantages offerts par les ISC dans la perspective globale de créer un impact, principes associés à trois objectifs principaux :

- Renforcer l'obligation de rendre compte, la transparence et la probité des organes gouvernementaux et du secteur public ;

- Faire constamment preuve de pertinence vis-à-vis des citoyens, du parlement et des parties prenantes;

- Être une organisation-modèle en montrant l'exemple.

Les principes s'articulent autour de l'attente fondamentale selon laquelle les ISC devraient changer les choses dans la vie des citoyens. Huit des douze principes de I'ISSAI 12 concernent la relation entre les parlements et les ISC, et chaque principe s'accompagne de plusieurs normes que les ISC devraient s'efforcer de respecter. Pendant l'élaboration de I'ISSAI 12, un document de travail a été présenté à I'XX INCOSAI d'Afrique du Sud en 2010. Reconnaître qu'une ISC est une institution qui change les choses dans la vie des citoyens a été défini comme une exigence fondamentale, en veillant à ce que les gouvernements soient tenus de rendre compte de l'utilisation régulière et responsable des ressources, pour les objectifs prévus, dans un souci d'économie, d'efficience et d'efficacité. Le concept suivant a été reconnu comme raison fondamentale de l'existence des ISC : fournir une garantie et des informations crédibles aux parties prenantes dans l'intérêt du public.

Parmi les normes essentielles de I'ISSAI 12 qui concernent la relation entre les ISC et leur parlement, citons :

La norme 12.2.4 : Les ISC devront, en vertu de leur mandat, soumettre les rapports de vérification comptable à l'assemblée législative ou tout autre organe public responsable, selon le cas. Cette norme est semblable à I'ISSAI 1 , section 16 , sous-section 1 . II est naturellement primordial que dans un système parlementaire, I'ISC soumette ses rapports au parlement. L'ISSAI 1 se concentre sur le mandat et l'obligation juridique tandis que I'ISSAI 12 part de la mission de I'ISC.

La norme 12.3.2 : Les ISC devront, en vertu de leur mandat, fournir à l'assemblée législative, à ses commissions ou à la direction et aux conseils d'administration des organismes contrôlés, les informations pertinentes et opportunes. Cette norme définit les exigences quant à la qualité des informations fournies par I'ISC : objectives, pertinentes et en temps voulu. Bien que la formulation se réfère au mandat des ISC, la norme suggère que, dès que cela est possible, I'ISC doit fournir au parlement des informations et soumettre des rapports dès que ces derniers sont prêts : Cette exigence fait écho à I'ISSAI 20, Principe 8, qui déclare entre autres: les ISC entreprennent et réalisent des audits et publient les rapports afférents en temps voulu. La transparence et la 
responsabilité seront renforcées si les activités d'audit et les informations qui en découlent ne sont pas obsolètes.

La norme 12.3.5 : Les ISC devront développer des relations professionnelles avec les commissions de supervision législatives ainsi qu'avec les directions et conseils d'administration des organismes contrôlés en vue de les aider à mieux comprendre les rapports de contrôle et les recommandations et prendre les mesures qui s'imposent. Cette norme précise que la soumission formelle des rapports peut ne pas suffire pour encourager un suivi et des mesures adéquats. Les ISC doivent faire davantage : présenter l'environnement et le contexte, proposer des explications techniques, prendre des initiatives, être proactives, concevoir et mettre en œuvre une stratégie de communication efficace.

La norme 12.4.3 : Les ISC devront faciliter l'accès à leurs rapports par l'ensemble des parties prenantes en utilisant les outils de communication appropriés. Cette norme renvoie à I'ISSAI 20, Principe 8, qui comporte des lignes directrices additionnelles. La note d'explication qui s'y rapporte répertorie plusieurs bonnes pratiques, telles que l'organisation de conférences de presse, la mise à disposition de rapports ainsi que d'autres informations sur le site Internet de l'ISC.

La norme 12.5.1 : Les ISC devront prendre conscience des attentes des parties prenantes et leur répondre, selon le cas, de manière opportune et sans compromettre leur indépendance ; la norme 12.5.2 : Les ISC devront, dans le cadre de l'élaboration de leur programme de travail, répondre de manière adaptée aux questions-clés ayant un impact sur la société ; et la norme 12.5.4. : Les ISC devront s'assurer que les attentes des parties prenantes et les risques émergents soient pris en compte dans les plans stratégiques, d'entreprises et de vérification comptable, de manière adaptée. Ces normes mettent l'accent sur l'importance que les ISC doivent attacher à la pertinence de leurs travaux pour les parties prenantes ; sur le fait qu'elles doivent répondre à leurs attentes, et qu'elles doivent prendre en compte ces éléments dans leur programme prévisionnel. Sans compromettre leur indépendance, elles invitent clairement les ISC à coopérer et à communiquer de manière efficace avec le parlement afin de comprendre ses attentes et d'y répondre.

La norme 12.6.1 : Les ISC devront communiquer de manière à améliorer la connaissance et la compréhension par les parties prenantes des rôles et responsabilités des ISC en tant que contrôleurs indépendants des finances du secteur public; la norme 12.6.2 : La communication des ISC devra contribuer à la prise de conscience par les parties prenantes du besoin de transparence et de reddition des comptes dans le secteur public ; la norme 12.6.3 : Les ISC devront communiquer avec les parties prenantes en vue d'assurer la compréhension du travail de vérification des ISC et des résultats ; et la norme 12.6 .5 : Les ISC devront engager les parties prenantes, par la reconnaissance de leurs différents rôles et tenir compte de leurs points de vue sans compromettre leur propre indépendance. Ces normes font référence à une communication efficace avec les parties prenantes, entre autres avec le parlement. L'ISC doit veiller à une bonne compréhension de ses travaux et de son rôle, et sensibiliser les parties prenantes au besoin de rendre des comptes. L'engagement requis oblige I'ISC à jouer un rôle actif envers ses parties prenantes, toutes ses actions visant à renforcer son impact. Cela peut notamment impliquer d'examiner attentivement les suggestions ou les demandes portant sur des sujets d'audit potentiels.

La norme 12.6.6 : Les ISC devront régulièrement vérifier si les parties prenantes jugent que leur communication est efficace ; et la norme 12.7.4 : Les ISC devront régulièrement vérifier si les parties prenantes reconnaissent leur efficacité et leur contribution aux améliorations dans l'administration publique. Ces deux normes évoquent l'obligation qu'ont les ISC d'évaluer leur 
efficacité en général et l'efficacité de leur communication en particulier. En règle générale, ce type de vérification peut être réalisé dans le cadre d'une évaluation par les pairs, mais d'autres instruments sont également possibles, tels que des questionnaires, des entretiens ou un examen externe par des universitaires ou des consultants experts. Il est important pour les ISC de connaître les attentes des parlementaires à leur égard afin de les prendre en compte ou bien, si nécessaire, de les gérer.

La norme 12.8.1 : Les ISC devront exécuter leurs fonctions de manière à intégrer la reddition des comptes, la transparence et la bonne gouvernance des finances publiques ; la norme 12.8.5 : Les ISC devront faire l'objet d'un examen externe indépendant y compris une vérification interne de leurs opérations et mettre ces rapports à disposition des parties prenantes ; la norme 12.9.1: Les ISC devront adopter et se conformer aux principes de bonne gouvernance et rendre leurs comptes sur cette base et de manière appropriée. Ces normes évoquent l'obligation qu'ont les ISC de rendre compte et d'être responsables de leur performance et de leur utilisation des ressources, et ceci y compris vis-à-vis du parlement.

\section{ISSAI 20 - Principes de transparence et de responsabilité}

En vertu du Principe 6 de l'ISSAI 20 qui invite les ISC à rendre compte publiquement de leur performance et de l'utilisation des ressources, les sous-principes stipulent : Les rapports financiers des ISC sont rendus publics et soumis à un audit externe indépendant ou à l'examen du parlement; et les ISC peuvent aussi utiliser des indicateurs de performance afin d'évaluer la qualité des audits pour le parlement, les citoyens et les autres parties prenantes. Ces préceptes traduisent le rôle imparti au parlement dans l'obligation qu'ont les ISC de rendre compte de leurs activités.

Le Principe 7 stipule que les ISC rendent publics les résultats de leurs audits et leurs conclusions concernant l'ensemble des activités gouvernementales :

- Les ISC rendent publiques les conclusions et les recommandations formulées au terme des audits, sous réserve que celles-ci ne soient pas considérées comme confidentielles en vertu de lois et réglementations spécifiques.

- Les ISC rendent compte des mesures prises à la suite de leurs recommandations.

- Les ISC du modèle juridictionnel rendent compte des sanctions et des pénalités imposées aux comptables ou aux gestionnaires.

- Par ailleurs, les ISC rendent publiques les conclusions d'audits plus globaux portant par exemple sur l'exécution du budget général, sur la situation financière de l'État et ses opérations, ainsi que, si cela fait partie de leurs compétences, sur les aptitudes professionnelles des agents publics.

- Les ISC entretiennent d'étroites relations avec les commissions parlementaires concernées afin de les aider à mieux comprendre les rapports et les conclusions d'audit et à prendre les mesures appropriées.

Dans la présentation jointe des Bonnes pratiques (ISSAI 21), il est fait mention du fait que les ISC fournissent des directives et dispensent une formation en gestion financière aux parlementaires, mettent à disposition des parlementaires un guide sur l'examen des dépenses publiques, et tiennent des réunions informelles avec les présidents et les membres des commissions parlementaires afin d'expliquer le rôle et le mandat de I'ISC et de mieux comprendre les attentes des différentes commissions. 


\section{Cadre de mesure de la performance des ISC}

Dans le cadre de I'ISSAI 12, le groupe de travail INTOSAI sur la valeur et les avantages des ISC a élaboré un Cadre de mesure de la performance (PMF). Ce cadre a été testé dans plusieurs régions de I'INTOSAI, la version définitive ayant été approuvée lors de I'INCOSAI 2016 à Abu Dhabi. Le PMF comporte un volet portant sur la communication avec les branches législative, exécutive et judiciaire. À cet égard, I'ISC doit s'efforcer de mettre en œuvre les Bonnes pratiques suivantes :

a) Elle tient compte des questions posées pendant les débats publics au parlement lorsqu'elle établit son plan de contrôle global, sans avoir besoin qu'une demande officielle lui soit adressée.

b) Elle recherche les opportunités, dans le cadre des usages et des pratiques du pays, pour dialoguer avec le parlement et les membres des commissions concernées, et présenter des rapports d'audit et des résultats pertinents.

c) Elle contribue au processus législatif en proposant des recommandations ou des amendements visant à améliorer le système de gestion des finances publiques.

d) Lorsqu'elle y est invitée, elle permet à l'assemblée législative d'accéder en temps voulu aux informations concernant ses activités.

e) Selon le cas, elle s'enquiert de l'opinion qu'ont les parlementaires et les commissions concernées sur sa performance.

f) Elle développe une relation professionnelle avec la commission parlementaire de surveillance compétente pour l'aider à mieux comprendre les rapports d'audit et leurs conclusions et à prendre les mesures qui s'imposent.

g) Elle sensibilise le parlement sur son rôle et son mandat.

h) Elle établit des politiques et des procédures destinées à définir sa communication avec le parlement.

Stapenhurst, Rick et al. (2014), Following the Money: Comparing Parliamentary Public Accounts Committees $^{29}$ présente certaines caractéristiques visant à obtenir la commission des comptes publics idéale :

- La commission est de taille réduite ; il semble que les commissions fonctionnent bien avec 5 à 11 membres ; elle ne doit pas compter de ministres du gouvernement.

- Les personnalités majeures de l'opposition sont associées aux travaux de la PAC, et il est probable que la présidence de la commission lui revient.

- Son président est un parlementaire chevronné. Il est équitable et respecté par le parlement.

- La commission est nommée pour toute la durée de la législature.

- La commission dispose de suffisamment de personnel, avec un secrétariat expérimenté et un ou plusieurs chercheurs compétents.

- Le rôle et les responsabilités de la commission sont clairement définis.

- La commission se réunit souvent et à intervalles réguliers. 
- Les auditions sont ouvertes au public ; une transcription verbatim intégrale des auditions et un résumé sont rapidement disponibles pour diffusion publique.

- Un comité directeur planifie les travaux de la commission et prépare l'agenda de chaque réunion plénière.

- Les personnes habituellement auditionnées sont des fonctionnaires de rang élevé (les " ordonnateurs») accompagnés par des agents qui connaissent dans le détail les points examinés.

- Le rapport de l'auditeur est transmis automatiquement à la commission et l'auditeur se réunit avec la commission pour examiner les points essentiels du rapport.

- Outre les problématiques évoquées par l'auditeur, la commission décide de temps à autre de conduire des investigations sur d'autres sujets.

- La commission s'efforce d'obtenir un consensus dans ses rapports.

- Au minimum une fois par an, la commission établit des rapports substantiels officiels à l'intention du parlement.

- En concertation avec le gouvernement, la commission a élaboré une procédure de suivi de ses recommandations, et est tenue informée des mesures prises, le cas échéant.

- Dans toutes les délibérations de la commission, l'auditeur sert de conseiller expert.

- Le parlement organise un débat annuel portant sur les activités de la commission.

Buzaljko, Karolina et al. (2010), Public Financial Oversight; A Comparative Analysis of Parliamentary Committees Across Europe

Les pages 56 à 60 de ce rapport de recherche sur la supervision des finances publiques ${ }^{30}$ résument les conclusions dont certaines peuvent servir de critères pour des relations de travail efficaces du point de vue du parlement.

1. Le mandat des commissions des finances publiques (PFC, terme se référant aux différents types de commissions qui examinent habituellement les rapports des ISC, telles que les commissions budgétaires ou les commissions de contrôle des comptes publics) doit être clairement défini et avoir un vaste périmètre.

2. Dans la composition des PFC, il faut penser en particulier à intégrer des membres de l'opposition.

3. La continuité des travaux des PFC d'un mandat parlementaire au suivant est essentielle pour s'assurer qu'une suite adéquate est donnée aux rapports de I'ISC.

4. Il est important que les membres et le président des PFC jouent un rôle actif.

5. Il est utile de dispenser une formation aux membres des PFC en début de législature et pendant leur mandat.

6. Les PFC doivent disposer d'un personnel et de ressources adéquats. 
7. Les PFC doivent se réunir à une fréquence qui leur permet d'analyser soigneusement les rapports des ISC.

8. Les PFC doivent établir des mécanismes de suivi bien définis, qui complètent ceux de l'ISC.

9. Les PFC doivent autant que possible faire preuve de transparence ; leurs réunions et leurs rapports doivent être publics avec, comme résultat, l'imposition d'une pression maximale sur l'exécutif afin d'améliorer la gestion et le contrôle des finances publiques.

10. Les PFC doivent rédiger un rapport annuel sur l'état de la supervision des finances publiques et évaluer leur propre performance dans le but d'améliorer leur fonctionnement. 


\section{ANNEXE 2. BOÎTE À OUTILS DESTINÉE À RENFORCER LES RELATIONS DE TRAVAIL ENTRE LES ISC ET LE PARLEMENT}

\begin{tabular}{|c|c|c|}
\hline & Exigences ISC & Bonnes pratiques \\
\hline \multirow[t]{4}{*}{ Rapports } & $\begin{array}{l}\text { Rapports soumis au parlement puis publiés. ISSAI 1, 10, } 12 \text { et } \\
20\end{array}$ & \multirow{4}{*}{$\begin{array}{l}\text { - } \quad \text { Soumettre au parlement les rapports et les publier simultanément. } \\
\text { - } \quad \text { Diffuser des communiqués de presse pour mettre en lumière les } \\
\text { - } \quad \text { Ruestions importantes. } \\
\text { Regrouper dans des rapports thématiques les résultats d'audits } \\
\text { précents. }\end{array}$} \\
\hline & $\begin{array}{l}\text { Utilisation des outils de communication appropriés pour } \\
\text { accéder facilement aux rapports et en faciliter la } \\
\text { compréhension. ISSAI } 12 \text { et } 20\end{array}$ & \\
\hline & $\begin{array}{l}\text { Fournir en temps voulu des informations objectives et } \\
\text { pertinentes à l'assemblée législative. ISSAI } 12\end{array}$ & \\
\hline & $\begin{array}{l}\text { Les rapports d'audit identifient les thématiques, les } \\
\text { constatations récurrentes, les tendances, les causes primaires } \\
\text { et les recommandations d'audit; ils sont étudiés avec les } \\
\text { principales parties prenantes. ISSAI } 12\end{array}$ & \\
\hline \multirow[t]{4}{*}{$\begin{array}{l}\text { Communication } \\
\text { - rôle et activités } \\
\text { des ISC }\end{array}$} & $\begin{array}{l}\text { Créer de bonnes relations de travail, établir des politiques et } \\
\text { des procédures de communication en liaison avec le } \\
\text { parlement. ISSAI } 12 \text { et } 20\end{array}$ & \multirow{4}{*}{$\begin{array}{l}\text { - } \quad \text { Élaborer une politique de communication. } \\
\text { Établir avec le parlement un protocole d'accord définissant les } \\
\text { procédures de coopération. } \\
\text { Organiser régulièrement des réunions avec le parlement, complétées par } \\
\text { des échanges informels au niveau opérationnel. } \\
\text { - } \quad \text { Maintenir le contact avec la commission spécialisée et les autres } \\
\text { commissions concernées. } \\
\text { - } \quad \text { 'assurer que les rapports sont distribués aux } \\
\text { - } \quad \text { Parlementaires/commissions concernés. } \\
\text { - Sélectionner éventuellement les rapports soumis ou faire des suggestions } \\
\text { - } \quad \text { Organ les apports à sélectionner pour examen. } \\
\text { Organiser des réunions de travail, des tables rondes et des ateliers. }\end{array}$} \\
\hline & $\begin{array}{l}\text { Coopérer avec le parlement et ses commissions, et les } \\
\text { sensibiliser au rôle de I'ISC. ISSAI } 12 \text { et } 20\end{array}$ & \\
\hline & $\begin{array}{l}\text { Aider le parlement à mieux comprendre les rapports d'audit. } \\
\text { ISSAI } 12 \text { et } 20\end{array}$ & \\
\hline & $\begin{array}{l}\text { Formuler des conseils sur la manière d'utiliser au mieux les } \\
\text { constatations et les opinions d'audit. ISSAI } 12 \text { et } 20\end{array}$ & \\
\hline
\end{tabular}




\begin{tabular}{|c|c|c|}
\hline & Exigences ISC & Bonnes pratiques \\
\hline & & $\begin{array}{l}\text { - } \quad \text { Publier du matériel et des livrets d'orientation. } \\
\text { Dispenser des programmes de formation / d'orientation sur I'ISC à } \\
\text { l'intention des nouveaux parlementaires. } \\
\text { Améliorer la compréhension mutuelle à travers le détachement de } \\
\text { personnel. }\end{array}$ \\
\hline \multirow{3}{*}{$\begin{array}{l}\text { Communication } \\
\text { - pertinence vis-à- } \\
\text { vis des parties } \\
\text { prenantes }\end{array}$} & $\begin{array}{l}\text { Connaître les attentes des parties prenantes et réagir à leurs } \\
\text { points de vue, sans compromettre son indépendance. ISSAI } 12\end{array}$ & \multirow{3}{*}{$\begin{array}{l}\text { - Convenir avec le parlement d'une procédure de consultation avant la } \\
\text { demande d'audit officielle. } \\
\text { - } \quad \text { Limiter le nombre d'audits à réaliser sur demande, simultanément ou } \\
\text { tout au long de l'année. } \\
\text { - } \quad \text { Informer le parlement du programme de travail (si la loi le permet). } \\
\text { Donner au parlement l'occasion d'apporter régulièrement une } \\
\text { contribution (réunions, entretiens). } \\
\text { Suivre les discussions parlementaires pertinentes afin de définir les audits } \\
\text { potentiels. }\end{array}$} \\
\hline & $\begin{array}{l}\text { Veiller à prendre en compte les attentes et les points de vue } \\
\text { des parties prenantes dans l'organisation et la planification de } \\
\text { ses contrôles. ISSAI } 12\end{array}$ & \\
\hline & $\begin{array}{l}\text { Évaluer l'opinion des parties prenantes sur l'efficacité de I'ISC } \\
\text { et sa contribution à l'amélioration du secteur public. ISSAI } 12\end{array}$ & \\
\hline \multirow[t]{3}{*}{$\begin{array}{l}\text { Communication - } \\
\text { divers }\end{array}$} & $\begin{array}{l}\text { La communication contribue à sensibiliser les parties prenantes } \\
\text { au besoin de transparence et de responsabilité dans le secteur } \\
\text { public. ISSAI } 12\end{array}$ & \multirow{3}{*}{$\begin{array}{l}\text { - } \quad \text { Rédaction et publication de rapports annuels sur les activités et la mise en } \\
\text { - } \quad \text { Réalisation programme de travail. } \\
\text { rapports sont mis à disposition du public. } \\
\text { Regrouper dans des rapports thématiques les résultats d'audits } \\
\text { précédents. }\end{array}$} \\
\hline & $\begin{array}{l}\text { Évaluation périodique de l'efficacité de la communication de } \\
\text { I'ISC. ISSAI } 12\end{array}$ & \\
\hline & $\begin{array}{l}\text { Favoriser le débat sur les améliorations à réaliser dans le } \\
\text { secteur public. ISSAI } 12\end{array}$ & \\
\hline \multirow[t]{2}{*}{ Suivi } & $\begin{array}{l}\text { Soumettre des rapports de suivi au parlement. ISSAI 10, } 12 \text { et } \\
20\end{array}$ & \multirow{2}{*}{$\begin{array}{l}\text { - Les recommandations sont solidement étayées, pratiques, réalistes et } \\
\text { - } \quad \text { Concréation d'un mécanisme de pilotage }{ }^{2} \text {. } \\
\text { - } \quad \text { Publication de l'état de mise en œuvre des recommandations. } \\
\text { - } \quad \text { 'état de la mise en œuvre des recommandations est communiqué à } \\
\text { intervalles fixes. } \\
\text { - } \quad \text { ve pilotage est intégré au système de planification des audits de } \\
\text { - Une attention particulière est accordée à la communication portant sur la } \\
\text { mise en œuvre des recommandations formulées dans les rapports }\end{array}$} \\
\hline & $\begin{array}{l}\text { Piloter et suivre les recommandations faites par l'ISC et le } \\
\text { parlement. ISSAI } 10 \text { et } 20 \text {, Principes applicables à } \\
\text { l'administration publique - Principe } 16\end{array}$ & \\
\hline
\end{tabular}




\begin{tabular}{|c|c|c|}
\hline & Exigences ISC & Bonnes pratiques \\
\hline & & d'audit de performance. \\
\hline \multirow{2}{*}{$\begin{array}{l}\text { Rapports relatifs à } \\
\text { la performance de } \\
\text { I'ISC }\end{array}$} & Soumettre au parlement un rapport d'activité annuel. ISSAI 10 & \multirow{2}{*}{$\begin{array}{l}\text { - Publication de rapports annuels certifiés portant sur les comptes et } \\
\text { - } \quad \text { l'utilisation des ressources. } \\
\text { Rédaction et publication de rapports annuels sur les activités et la mise en } \\
\text { - } \quad \text { Réalisation périodique d'évaluations externes par les pairs, dont les } \\
\text { rapports sont mis à disposition du public. } \\
\text { - } \quad \text { Estimation des économies obtenues dans le secteur public. }\end{array}$} \\
\hline & $\begin{array}{l}\text { L'ISC doit être soumise à un examen externe et le résultat doit } \\
\text { être communiqué aux parties prenantes. ISSAI } 12 \text { et } 20\end{array}$ & \\
\hline
\end{tabular}

\begin{tabular}{|c|c|c|}
\hline & Actions attendues des parlements & Bonnes pratiques \\
\hline $\begin{array}{l}\text { Obligation de } \\
\text { rendre compte }\end{array}$ & $\begin{array}{l}\text { Le parlement oblige le gouvernement à rendre compte. Les } \\
\text { Principes de l'administration publique et le PEFA }\end{array}$ & $\begin{array}{l}\text { - Mise en place de procédures normalisées destinées au traitement des } \\
\text { rapports d'audit. } \\
\text { - Organisation d'auditions en présence des entités contrôlées. } \\
\text { - } \quad \text { Demande d'un plan d'action au gouvernement ou à l'entité contrôlée. } \\
\text { Des dates butoir sont associées aux plans d'action ou aux mesures à } \\
\text { prendre. } \\
\text { Des sanctions (politiques, financières et disciplinaires) sont considérées } \\
\text { par l'ISC ou le parlement en cas de non-respect grave des } \\
\text { recommandations. } \\
\text { Obligation des entités contrôlées ou du gouvernement de fournir des } \\
\text { rapports de mise en œuvre. }\end{array}$ \\
\hline \multirow[t]{3}{*}{$\begin{array}{l}\text { Traitement des } \\
\text { rapports des ISC }\end{array}$} & $\begin{array}{l}\text { Le parlement dispose d'un mécanisme formalisé pour examiner } \\
\text { les rapports de l'ISC. Les Principes de l'administration publique - } \\
\text { Principe } 16 \text { et le PEFA }\end{array}$ & \multirow{3}{*}{$\begin{array}{l}\text { - Les responsabilités de la commission chargée de l'examen des rapports de } \\
\text { - } \quad \text { l'ISC sont organisées de manière adéquate. } \\
\text { - Des commissions sectorielles participent au traitement des rapports } \\
\text { d'audit de performance. } \\
\text { - Une procédure de décharge formalisée fait partie du cycle budgétaire } \\
\text { dans le cadre de la supervision budgétaire. } \\
\text { - Nomination d'un rapporteur pour certains rapports de l'ISC. } \\
\text { Coordination avec le parlement sur les ordres du jour et les calendriers } \\
\text { de soumission des rapports. }\end{array}$} \\
\hline & $\begin{array}{l}\text { Le parlement examine les rapports d'audit en temps voulu et } \\
\text { formule des conclusions ou recommandations. PEFA }\end{array}$ & \\
\hline & $\begin{array}{l}\text { Le parlement suit la mise en œuvre des recommandations par } \\
\text { le gouvernement. PEFA }\end{array}$ & \\
\hline
\end{tabular}




\begin{tabular}{|l|l|l|}
\hline & & $\begin{array}{l}\text { Fixation d'un calendrier des débats parlementaires portant sur } \\
\text { les rapports de l'ISC pour pouvoir rendre les conclusions en temps opport } \\
\text { Mise à la disposition du parlement d'un personnel et de capacités } \\
\text { d'analyse suffisantes. }\end{array}$ \\
\hline
\end{tabular}




\section{ANNEXE 3. QUESTIONNAIRE}

\section{1) Cadre juridique :}

a) Pourriez-vous décrire le cadre juridique dans lequel s'inscrivent les obligations de l'ISC envers le parlement (comme par exemple la communication du programme de travail de I'ISC, les obligations de rendre compte de I'ISC, et la nécessité de prendre en considération les audits demandés par le parlement) ? Prenez en compte les dispositions de la Constitution et de la loi relative au contrôle des comptes publics et, le cas échéant, de la loi de procédure budgétaire ou loi équivalente.

\section{2) Modalités de soumission des rapports de l'ISC au parlement :}

a) Décrivez le type de rapports que I'ISC envoie au parlement, par exemple des rapports d'audit financier, d'audit de conformité, d'audit de performance, des avis, des rapports (d'activité) annuels / trimestriels (incluant les rapports sur les travaux-mêmes de I'ISC, sur la façon dont elle a exécuté son mandat). Veuillez également indiquer :

- Si I'ISC rend compte séparément des audits individuels ou si les résultats de plusieurs audits sont réunis au sein d'un seul rapport ;

- À qui les rapports sont adressés (le président du parlement et / ou le président de la commission spécialisée ${ }^{31}$ ) et si des copies sont envoyées (ou non) aux autres commissions concernées ;

- Si la soumission des rapports au parlement s'inscrit dans un calendrier ou est assortie de délais.

b) L'envoi des rapports au parlement est-il régi par des procédures spécifiques ? L'ISC utilise-t-elle d'autres moyens de communication concernant ses rapports, tels que des notes de synthèse ou des présentations au parlement / à la commission parlementaire ?

c) Veuillez compléter le tableau suivant afin de fournir des informations sur le nombre et le type de rapports que I'ISC a soumis au parlement en 2015. Si la classification en rapports financiers, de conformité et de performance n'est pas appropriée à la façon dont votre institution rend compte de son travail de vérification au parlement, précisez néanmoins le nombre de rapports d'audit soumis, puis expliquez comment l'ISC rend compte de son travail de vérification. 


\begin{tabular}{|c|c|c|c|c|c|c|c|}
\hline & $\begin{array}{l}\text { Rapports } \\
\text { d'audit } \\
\text { financier }\end{array}$ & \begin{tabular}{|} 
Rapports d'audit \\
de conformité
\end{tabular} & $\begin{array}{l}\text { Rapports d'audit } \\
\text { de performance }\end{array}$ & $\left|\begin{array}{c}\text { Autres rapports } \\
\text { ou documents }\end{array}\right|$ & $\begin{array}{c}\text { Rapports } \\
\text { d'activité } \\
\text { annuels }\end{array}$ & $\begin{array}{c}\text { Rapports à la } \\
\text { demande du } \\
\text { parlement }\end{array}$ & Remarques \\
\hline $\begin{array}{c}\text { Nombre de } \\
\text { rapports soumis }\end{array}$ & & & & & & & \\
\hline $\begin{array}{l}\text { Nombre de } \\
\text { rapports discutés } \\
\text { par le parlement }\end{array}$ & & & & & & & \\
\hline $\begin{array}{l}\text { Soumis au } \\
\text { président }\end{array}$ & $\mathrm{o} / \mathrm{n}$ & $\mathrm{o} / \mathrm{n}$ & $\mathrm{o} / \mathrm{n}$ & $\mathrm{o} / \mathrm{n}$ & $\mathrm{o} / \mathrm{n}$ & $\mathrm{o} / \mathrm{n}$ & \\
\hline $\begin{array}{l}\text { Soumis au } \\
\text { président de la } \\
\text { commission } \\
\text { spécialisée }\end{array}$ & $0 / n$ & $\mathrm{o} / \mathrm{n}$ & $\mathrm{o} / \mathrm{n}$ & $0 / n$ & $\mathrm{o} / \mathrm{n}$ & $\mathrm{o} / \mathrm{n}$ & \\
\hline $\begin{array}{l}\text { Copies envoyées } \\
\text { aux autres } \\
\text { commissions } \\
\text { concernées }\end{array}$ & $\mathrm{o} / \mathrm{n}$ & $\mathrm{o} / \mathrm{n}$ & $o / n$ & $\mathrm{o} / \mathrm{n}$ & $\mathrm{o} / \mathrm{n}$ & $\mathrm{o} / \mathrm{n}$ & \\
\hline $\begin{array}{c}\text { Notes de synthèse } \\
\text { destinées à la } \\
\text { commission } \\
\text { parlementaire }\end{array}$ & $\mathrm{o} / \mathrm{n}$ & $\mathrm{o} / \mathrm{n}$ & $\mathrm{o} / \mathrm{n}$ & $\mathrm{o} / \mathrm{n}$ & $\mathrm{o} / \mathrm{n}$ & $\mathrm{o} / \mathrm{n}$ & \\
\hline $\begin{array}{c}\text { Résumés soumis au } \\
\text { parlement }\end{array}$ & $\mathrm{o} / \mathrm{n}$ & $\mathrm{o} / \mathrm{n}$ & $\mathrm{o} / \mathrm{n}$ & $\mathrm{o} / \mathrm{n}$ & $\mathrm{o} / \mathrm{n}$ & $\mathrm{o} / \mathrm{n}$ & \\
\hline
\end{tabular}

3) Procédures parlementaires utilisées dans le traitement des rapports de l'ISC :

a) Pourriez-vous décrire les procédures appliquées par le parlement pour examiner les rapports de I'ISC ? Dans vos réponses, merci d'indiquer :

- Dans le cadre du traitement des rapports de I'ISC, si des procédures établies sont inscrites dans les règles de procédure du Parlement ou dans un document du même type ;

- S'il existe une commission dédiée au traitement des rapports de I'ISC, et si elle est présidée par un membre de l'opposition ou par un membre de la majorité gouvernementale, et si cette présidence est fondée sur la tradition, une procédure ou des négociations politiques ;

- Si la commission nomme un rapporteur, par exemple pour un rapport d'audit donné ;

- S'il existe des auditions auxquelles est conviée l'entité contrôlée ou d'autres personnes concernées afin d'apporter des clarifications ou un complément d'informations ; 
- Si la commission peut, si nécessaire, faire examiner les rapports par des experts externes indépendants, ou si elle peut demander par écrit aux personnes appropriées un complément d'informations ou des clarifications ;

- Si le débat sur les rapports de l'ISC se déroule au sein d'une (de) commission(s) et / ou en séance plénière ;

- Si des commissions autres que les commissions compétentes examinent également les rapports de I'ISC ;

- Si un "produit » parlementaire, par exemple une résolution ou un compte-rendu, résulte du débat sur le rapport de l'ISC, et si ces " produits » sont publiés ;

- Si le Parlement possède une procédure de décharge bien établie, si elle est appliquée et si l'ISC et / ou ses rapports jouent un rôle dans cette procédure.

b) Veuillez indiquer le nombre et le niveau du personnel parlementaire qui assiste la ou les commissions affectées au traitement des rapports de I'ISC. Merci de préciser également leur fonction, par exemple :

- Fournissent-ils des documents de recherche écrits, des analyses ou des notes d'informations dans le but de préparer les débats sur les rapports de l'ISC ?

- Élaborent-ils des questions que la ou les commissions poseront durant les auditions ?

- Rédigent-ils des projets de rapports ou d'autres documents que la ou les commissions doivent produire?

c) Veuillez fournir des informations sur la participation de I'ISC à l'examen de ses rapports par le parlement. Indiquez par exemple si I'ISC :

- Participe à la préparation des auditions aux côtés de la commission ou du parlement ;

- Assiste aux réunions parlementaires lorsque ses rapports sont débattus ; indiquez à quel niveau hiérarchique l'ISC est représentée, et quel est le type ou le degré de contribution qu'elle apporte aux réunions.

\section{4) Rapports de I'ISC :}

- Dans sa communication avec le parlement, l'ISC se concentre-t-elle sur des problèmes génériques / systémiques et sur la formulation de recommandations?

- La configuration et le fonctionnement des systèmes de contrôle internes sont-ils des sujets mis en exergue dans les rapports de l'ISC ?

- Tous les rapports d'audit contiennent-ils des conclusions et des opinions claires ?

5) Suivi des rapports de I'ISC :

a) Pourriez-vous décrire comment I'ISC pilote et assure le suivi des conclusions et des recommandations présentées dans ses rapports. Ce faisant, merci d'indiquer :

- Si I'ISC assure le suivi des conclusions et des recommandations acceptées par l'entité contrôlée, ainsi que les types d'outils utilisés à ces fins ;

- Si le parlement suit la mise en œuvre des recommandations que I'ISC formule dans ses rapports d'audit ; décrivez les mécanismes qu'il utilise à cette fin ; 
- Si I'ISC consacre un rapport spécifique à la mise en œuvre / l'absence de mise en œuvre des recommandations, y compris celles relatives à la configuration et au fonctionnement des systèmes de contrôle interne et le cas échéant, si cette démarche encourage le suivi parlementaire ;

- Si le gouvernement est obligé de rendre compte de la mise en œuvre des recommandations de I'ISC. Les rapports de I'ISC sont-ils régulièrement utilisés lors du débat annuel sur les projets de budget?

\section{6) Audits réalisés à la demande du parlement :}

a) Si une disposition juridique permet au parlement de solliciter des audits, veuillez présenter comment elle est mise en œuvre en pratique et la fréquence d'application de cette disposition. En l'absence de disposition juridique, le parlement demande-t-il néanmoins à I'ISC d'effectuer des audits sur des thèmes particuliers ou de donner des conseils et si tel est le cas, veuillez indiquer comment I'ISC répond à ces demandes.

\section{7) Programme de travail :}

a) L'ISC consulte-t-elle le parlement (commissions parlementaires, président du parlement, autres organes parlementaires, etc.) sur son programme de travail (pluri-)annuel en matière de contrôles ? Si tel est le cas, veuillez expliquer comment se déroule cette consultation, si le parlement émet des suggestions, et si l'ISC les prend sérieusement en considération.

8) Niveau et fréquence des échanges :

a) Veuillez décrire le niveau et la fréquence des échanges formels et informels entre I'ISC et le parlement. Précisez par exemple

- S'il y a régulièrement des échanges (incluant les échanges informels) entre le président de l'ISC et le président de la commission parlementaire compétente, et leur fréquence (mensuels, trimestriels, etc.) ;

- S'il y a des échanges au quotidien à un niveau opérationnel; et

- Si du personnel ou une unité de l'ISC est spécifiquement chargée des relations avec le parlement.

9) Bonnes pratiques :

a) Avez-vous des exemples précis que vous souhaiteriez signaler comme de bonnes pratiques applicables à la façon dont I'ISC gère ses relations avec le parlement afin d'accroître l'impact de ses travaux et de ses rapports?

\section{0) Perspective :}

a) Quels sont les points faibles de la relation actuelle, et qu'est-il prévu de faire pour traiter ces questions dans un avenir proche ? Pour y parvenir, comment la coopération entre I'ISC et le parlement est-elle envisagée?

11) Y-a-t-il d'autres éléments concernant les relations actuelles entre I'ISC et le parlement que vous souhaiteriez souligner, et quelle a été leur évolution au cours des cinq dernières années? 


\section{Contributions des ISC}

La contribution des pays a été transmise par les Institutions supérieures de contrôle des finances publiques des Pays membres de l'Union européenne et du Réseau en répondant à une enquête menée début 2016. SIGMA a mis en forme les textes, mais, par principe, n'en a pas modifié la substance; les ISC restent seules responsables du contenu de leur contribution. 


\section{ALBANIE}

Institution supérieure de contrôle

\section{1) Cadre juridique}

En vertu de l'Article 164 de la Constitution albanaise, I'Institution supérieure de contrôle d'Albanie (ALSAI) présente au Parlement albanais le rapport sur l'exécution du budget de l'État de l'année antérieure, une opinion sur le rapport du Conseil des ministres relatif aux dépenses budgétaires de l'année antérieure (avant que le Parlement n'approuve le budget de l'État de l'année à venir), des informations sur les résultats des audits à la demande du Parlement, et le rapport annuel sur ses activités.

L'obligation de I'ISC de remettre au Parlement les rapports susmentionnés est également incluse dans la loi relative à I'ISC.

\section{2) Modalités de soumission des rapports de l'ISC au parlement}

L'ALSAI envoie au Parlement le rapport sur l'exécution du budget de l'État pour l'année antérieure, accompagné d'une opinion sur le rapport du Conseil des ministres relatif aux dépenses budgétaires de l'année antérieure, avant que le Parlement n'approuve le budget de l'État de l'année à venir. Ce rapport contient les résultats de plusieurs audits financiers, d'audits de conformité, d'audits de performance et d'audits informatiques effectués par l'ALSAI pendant l'année concernée.

Par ailleurs, I'ALSAI envoie au Parlement un rapport annuel (le rapport de performance de I'ISC) sur ses audits et ses autres activités.

Les deux rapports susmentionnés contiennent les résultats de plusieurs audits regroupés dans un même rapport.

Ces deux rapports sont adressés au Président de la commission parlementaire spécialisée, la Commission de l'économie et des finances. Une copie est distribuée à tous les membres de la Commission, puis à tous les parlementaires, avant qu'ils ne discutent des rapports en séance plénière.

L'ALSAI présente le rapport sur l'exécution du budget de l'État début octobre, et son rapport de performance chaque année à la fin du mois d'avril.

Chaque rapport est introduit par une note d'information qui reprend les messages les plus importants du rapport.

Nombre et type de rapports

\begin{tabular}{|c|c|c|c|c|c|c|c|}
\hline & $\begin{array}{c}\text { Rapports d'audit } \\
\text { financier }\end{array}$ & $\begin{array}{c}\text { Rapports } \\
\text { d'audit de } \\
\text { conformité }\end{array}$ & $\begin{array}{l}\text { Rapports d'audit } \\
\text { de performance }\end{array}$ & $\begin{array}{c}\text { Autres rapports } \\
\text { ou documents }\end{array}$ & $\begin{array}{c}\text { Rapports } \\
\text { d'activité } \\
\text { annuels }\end{array}$ & $\begin{array}{c}\text { Rapports } \\
\text { demandés } \\
\text { par le } \\
\text { Parlement }\end{array}$ & Remarques \\
\hline $\begin{array}{c}\text { Nombre de } \\
\text { rapports soumis }\end{array}$ & $\begin{array}{c}\text { Non disponible } \\
\text { individuellement, } \\
\text { voir le rapport de } \\
\text { performance de }\end{array}$ & 1 & $\begin{array}{c}\text { Non disponible } \\
\text { individuellement, } \\
\text { voir le rapport de } \\
\text { performance de }\end{array}$ & $\begin{array}{c}\text { Rapport sur } \\
\text { l'exécution du } \\
\text { budget de l'État } \\
\text { de l'année }\end{array}$ & $\begin{array}{c}\text { Rapport de } \\
\text { performance } \\
\text { de l'ISC qui } \\
\text { contient les } \\
\text { résultats de }\end{array}$ & Sans objet & \\
\hline
\end{tabular}




\begin{tabular}{|c|c|c|c|c|c|c|}
\hline & I'ISC & & I'ISC & précédente & \begin{tabular}{|c|} 
tous les audits \\
financiers, de \\
conformité, de \\
performance \\
et des \\
systèmes \\
informatiques \\
réalisés en \\
2015
\end{tabular} & \\
\hline \begin{tabular}{|c|} 
Nombre de \\
rapports discutés \\
par le Parlement
\end{tabular} & & & & \begin{tabular}{|c|} 
Le rapport de \\
I'ALSAI sur \\
l'exécution du \\
budget de l'État \\
pour 2014 a été \\
discuté par le \\
Parlement lors de \\
la séance \\
plénière pendant \\
laquelle le \\
Parlement a \\
approuvé le \\
budget de l'État \\
pour 2016
\end{tabular} & \begin{tabular}{|c|} 
Le rapport de \\
performance \\
de I'ISC a été \\
discuté par la \\
commission de \\
I'économie et \\
des finances
\end{tabular} & \\
\hline $\begin{array}{l}\text { Soumis au } \\
\text { Président }\end{array}$ & Non disponible & 1 & Non disponible & Oui & Oui & Sans objet \\
\hline \begin{tabular}{|c|} 
Soumis au \\
Président de la \\
commission \\
spécialisée
\end{tabular} & Non disponible & 1 & Non disponible & Oui & Oui & Sans objet \\
\hline $\begin{array}{c}\text { Copies envoyées } \\
\text { aux autres } \\
\text { commissions } \\
\text { concernées }\end{array}$ & Non disponible & $\begin{array}{c}\text { Non } \\
\text { disponible }\end{array}$ & Non disponible & Oui & Oui & Sans objet \\
\hline $\begin{array}{c}\text { Notes de } \\
\text { synthèse } \\
\text { destinées à la } \\
\text { commission } \\
\text { parlementaire }\end{array}$ & Non disponible & $\begin{array}{c}\text { Non } \\
\text { disponible }\end{array}$ & Non disponible & Non disponible & Non disponible & Sans objet \\
\hline $\begin{array}{c}\text { Résumés soumis } \\
\text { au Parlement }\end{array}$ & Non disponible & $\begin{array}{c}\text { Non } \\
\text { disponible }\end{array}$ & Non disponible & Non disponible & Non disponible & Oui \\
\hline & & & & & & \\
\hline
\end{tabular}




\section{3) Procédures parlementaires concernant le traitement des rapports de l'ISC}

Une commission spécialisée traite les rapports de I'ISC. C'est la Commission de l'économie et des finances. Elle est présidée par un membre des partis du gouvernement, sélectionné par négociation.

Le débat sur les rapports de l'ISC a lieu au sein de la Commission de l'économie et des finances, et parfois en séance plénière. Les parlementaires abordent le rapport de I'ISC sur l'exécution du budget de l'État de l'année antérieure à la séance plénière qui adopte le budget de l'État pour l'année à venir. Les parlementaires examinent le rapport de performance de I'ISC lorsqu'ils discutent et prononcent en séance plénière une résolution parlementaire sur l'ISC.

La Commission spécialisée peut désigner un rapporteur pour un rapport d'audit particulier, mais cela ne s'est pas encore produit.

Pour examiner les rapports, la Commission spécialisée peut, si nécessaire, avoir recours à des experts externes indépendants ou bien demander par écrit des informations ou clarifications complémentaires, mais cela ne s'est pas encore produit.

La Commission spécialisée peut tenir des auditions lors desquelles l'entité contrôlée ou d'autres personnes concernées sont invitées à présenter des informations ou clarifications complémentaires, mais cela ne s'est pas encore produit.

Mise à part la Commission spécialisée (Commission de l'économie et des finances), d'autres commissions peuvent également analyser les rapports de I'ISC, mais cela ne s'est pas encore produit.

Un " Produit » parlementaire, une résolution sur la performance de I'ISC accompagnée de lignes directrices sur son fonctionnement futur, sont élaborés de temps à autre. La dernière résolution du 26 juin 2014 intitulée "Résolution pour l'évaluation de l'activité de l'ALSAI pour l'année 2013 » a été publiée dans le Journal officiel $N^{\circ} 101$ du 2 juillet 2014.

\section{Personnel}

Le Parlement ne dispose pas d'un personnel de soutien important pour ses commissions. La Commission de l'économie et des finances a un secrétaire qui rédige les minutes des réunions dédiées aux discussions sur les rapports, et ces minutes sont publiées sur le site Internet du Parlement.

\section{Participation de l'ISC à l'examen des rapports par le Parlement}

À la demande de la Commission spécialisée (Commission de l'économie et des finances) ou d'autres commissions, I'ALSAI peut préparer par écrit des documents / clarifications ou se réunir avec les représentants des commissions pour préparer ensemble les auditions, mais cette situation ne s'est pas encore produite.

L'ALSAI participe aux réunions parlementaires lorsque les rapports de I'ISC sont débattus en commission ou en séance plénière. L'ISC est normalement représentée par son responsable (le Président de l'ALSAI) accompagné du Secrétaire générale et du Directeur du service juridique. Ils répondent aux questions posées par les membres de la commission ou par les parlementaires en réunion plénière et ils fournissent les clarifications demandées. 


\section{4) Rapports de I'ISC}

Les rapports de I'ALSAI au Parlement se concentrent sur des problèmes et des recommandations génériques / systémiques. L'ALSAI aborde ces thèmes en deux fois, dans les notes d'information qui introduisent le rapport sur l'exécution du budget de l'État et le rapport de performance annuel de I'ISC, et sous forme plus détaillée dans les deux rapports.

La configuration et le fonctionnement des systèmes de contrôle interne est un sujet mis en avant dans les deux rapports de I'ISC, mais plus particulièrement dans le rapport portant sur l'exécution du budget de l'État. Les recommandations visant à améliorer le système et le fonctionnement du contrôle interne, ainsi que la qualité et la compétence des contrôleurs internes, sont les recommandations majeures des rapports de l'ISC.

L'ensemble des rapports d'audit contiennent des conclusions et des opinions d'audit claires.

\section{5) Suivi des rapports de I'ISC}

L'ALSAI conserve une trace des constatations, des conclusions et des recommandations acceptées par les entités contrôlées ainsi que les types d'outils qu'elles utilisent pour suivre l'avancement de la mise en œuvre. À chaque nouvel audit, la première chose que fait l'équipe chargée de l'audit consiste à vérifier le stade de la mise en œuvre des recommandations formulées dans le dernier rapport d'audit.

Le Parlement est attentif à la mise en œuvre des recommandations formulées dans les rapports d'audit de I'ISC, mais jusqu'à ce jour, le suivi réalisé par le Parlement n'est pas efficace, comme le soulignent les derniers rapports d'avancement de l'Union européenne sur l'Albanie.

L'ISC établit des rapports spécifiques et fréquents sur l'état de la mise en œuvre / de l'absence de mise en œuvre de ses recommandations, englobant les rapports concernant la configuration et le fonctionnement des systèmes de contrôle internes. Jusqu'à ce jour, les efforts faits pour que le Parlement suive les rapports n'ont pas été efficients.

Le Parlement n'a pas demandé au gouvernement de faire un rapport sur la mise en œuvre des recommandations de I'ISC. Les parlementaires puisent régulièrement dans ses rapports des informations qu'ils utilisent lors du débat annuel sur les projets de budget.

\section{6) Audits réalisés à la demande du Parlement}

Aucune disposition juridique ne permet au Parlement de demander des audits, mais l'ALSAI accorde une priorité absolue à toute demande ou suggestion du Parlement au regard de thématiques potentielles de contrôle ou d'informations complémentaires liées aux audits effectués. Ces requêtes et suggestions sont habituellement émises par la Commission spécialisée réunie pour discuter les rapports de l'ISC, mais elles peuvent également émerger de réunions informelles entre des parlementaires et le Président de I'ISC, ou avec tout autre fonctionnaire d'un grade élevé, ou bien encore faire suite à des déclarations ou des annonces des parlementaires aux médias.

\section{7) Programme de travail}

L'ALSAI consulte invariablement le Parlement sur son programme de travail (pluri-)annuel en matière de contrôles. Pour cela, elle invite les membres de la Commission spécialisée (Commission de l'économie et des finances) à participer à son analyse de performance annuelle, à ses activités visant à faire connaître ses travaux de contrôle et à sa Conférence scientifique annelle. 
L'ALSAI distribue ses publications aux membres de la Commission spécialisée (Commission de l'économie et des finances) par voie électronique ou sous forme papier. Sur les quatre dernières années (2012-2015), I'ALSAI a publié 50 livres.

Toute suggestion émanant d'un membre du Parlement est prise très au sérieux par l'ALSAI.

\section{8) Niveau et fréquence des échanges}

Des échanges réguliers (y compris des échanges informels) ont lieu entre le Président de I'ISC et le Président de la Commission parlementaire compétente (Commission de l'économie et des finances). Ces échanges ont normalement lieu une fois par mois.

II n'y a pas d'échange au quotidien, car les membres de la Commission spécialisée sont très occupés.

L'ISC n'a pas de personnel ou d'unité spécifiquement chargé des relations parlementaires, mais le Président de I'ISC entretient et développe ces relations. L'ALSAI est prête à affecter du personnel et à introduire une unité spécialement chargée des relations parlementaires ; toutefois, ce projet dépend de l'attitude du Parlement, à savoir s'il comprend l'importance de renforcer ses relations avec I'ISC. L'ALSAI s'emploie à faire davantage connaître sa mission auprès du Parlement.

\section{9) Bonnes pratiques}

Le long processus d'adoption de la nouvelle loi ALSAI en 2014, pleinement conforme aux normes INTOSAI (selon les déclarations de la DG Budget et de la délégation de l'UE à Tirana) est un bon exemple des efforts intensifs que fait I'ISC pour accroître son indépendance, ainsi que l'impact de son travail et de ses rapports.

\section{0) Perspective}

Peut être considéré comme point faible le fait que le travail et les rapports de I'ISC ne sont pas suffisamment connus par les autres commissions et les parlementaires en général.

L'ALSAI considère que partager des réunions de consultation et d'information avec les autres commissions est un outil efficace pour accroître les connaissances et la compréhension du Parlement au regard de I'ISC.

11) Y-a-t-il d'autres éléments concernant les relations actuelles entre l'ISC et le Parlement que vous souhaiteriez souligner, et quelles ont été leur évolution au cours des cinq dernières années?

En règle générale, le niveau de mise en œuvre des recommandations de I'ALSAI est satisfaisant, sauf en ce qui concerne la recommandation visant à révoquer du service civil les hauts fonctionnaires accusés par I'ISC d'avoir lésé les intérêts de l'État. Ce niveau de mise en œuvre est très bas. L'ALSAI souhaiterait un plus grand engagement du Parlement afin de forcer le gouvernement à mettre en œuvre ces recommandations, puisqu'elle lutte directement contre la "culture de l'impunité » qui règne dans le pays. 


\section{AUTRICHE}

Cour d'audit

\section{1) Cadre juridique}

La Cour d'audit autrichienne (ACA) examine les ressources publiques à l'échelon fédéral, des Länder et municipal pour juger si elles sont utilisées de manière économique, efficiente et efficace. Elle a pour mission essentielle d'offrir des services de contrôle et de conseil. Les activités, les fonctions et le statut de I'ACA sont gouvernés par le chapitre $\mathrm{VI}$ de la Loi constitutionnelle fédérale (http://www.rechnungshof.gv.at/en/austrian-court-of-audit/legal-provisions/the-title-five-of-the-federalconstitution-audit-of-financial-operations.html). Des informations complémentaires figurent dans les lois des Länder (http://www.rechnungshof.gv.at/en/aca/legal-provisions/laender-constitutions.html) et dans la Loi sur la Cour d'audit autrichienne (http://www.rechnungshof.gv.at/en/aca/legal-provisions/federalact-on-the-austrian-court-of-audit.html\#c659).

En vertu de l'Article $126 \mathrm{D}$ de la Loi constitutionnelle fédérale autrichienne, l'ACA doit rendre compte au Conseil national de ses activités de l'année précédente, au plus tard le 31 décembre (rapport d'activité). Les mêmes obligations valent pour les länder et les municipalités en vertu de l'Article 127 alinéa 6 et de l'Article 127 A alinéa 7 de la Loi constitutionnelle fédérale autrichienne. Les rapports de l'ACA sont publiés sur son site Internet après soumission au Conseil national, au Parlement du Land et au conseil municipal.

L'ACA soumet (au titre de l'Article 121 alinéa 2) au Conseil national un rapport sur les états financiers fédéraux annuels qui présente les recettes et les dépenses fédérales de l'exercice comptable précédent et qui permet d'évaluer la gestion budgétaire réelle.

La première délibération concernant les rapports de I'ACA a lieu au sein de la Commission de la Cour d'audit du Conseil national. Une fois que cette commission a examiné les rapports, ces derniers sont présentés au Conseil national réuni en séance plénière. Les mêmes dispositions valent pour les Länder et les municipalités (au parlement des Länder et au conseil municipal). Les règles de procédures du Conseil national et des parlements des Länder régissent la participation stratégiquement importante de l'ACA dans les réunions des commissions et en séances plénières. Selon l'Article 123 A de la Loi constitutionnelle fédérale autrichienne, le Président de I'ACA a le droit de participer aux débats du Conseil national et de ses commissions (et sous-commissions) sur les rapports publiés par l'ACA. Cette règle s'applique aux états financiers fédéraux, aux motions visant l'exécution de travaux d'audit particuliers et aux sections de la Loi des finances fédérales qui concernent les travaux de l'ACA. En vertu des dispositions détaillées des Règles de procédure du Conseil national, le président de l'ACA peut être entendu dans les débats sur ces sujets.

L'ACA sélectionne les thématiques à contrôler en toute indépendance. En vertu de l'Article 126 B alinéa 4, I'ACA doit, sur décision du Conseil national ou à la demande de ses membres, effectuer des travaux d'audit particuliers sur la gestion des finances, dans son domaine de compétences. Les Règles du Code de procédure du Conseil national fournissent des dispositions plus détaillées sur cette réglementation. De même, L'ACA doit réaliser ce type d'audit à la demande motivée du gouvernement ou d'un ministre fédéral, et soumettre un rapport sur les constatations du contrôle à l'autorité requérante. Des dispositions identiques existent aux niveaux des Länder et des municipalités. 


\section{2) Modalités de soumission des rapports de l'ISC au parlement}

L'ACA soumet des rapports d'audit de performance, de conformité et financiers à n'importe quelle période de l'année, et son rapport d'activité en fin d'année. Plusieurs audits sont réunis dans un groupe de rapports. Outre ces rapports, l'ACA soumet son rapport sur les revenus moyens et les pensions payées par les entreprises et les agences publiques fédérales, ainsi que son rapport sur le revenu moyen de l'ensemble de la population (rapport sur le revenu). L'ACA soumet également un rapport annuel et d'autres rapports sur des thématiques présentant un intérêt majeur (comme récemment l'avis sur l'amélioration de l'administration scolaire).

Les rapports sont distribués au Parlement et, selon le besoin, à toutes les autorités compétentes. En plus d'informer les parlementaires (MEP) qui siègent à la Commission de la Cour d'audit, I'ACA informe également tous ceux qui appartiennent à d'autres commissions parlementaires en fonction du sujet du rapport (par exemple les MEP de la Commission des transports si le rapport traite des transports). Après les avoir distribués au Parlement, les rapports sont publiés sur le site Internet de l'ACA.

L'ACA soumet au Conseil national les comptes des finances fédérales annuels au plus tard le 30 juin, et son rapport d'activité en fin d'année.

\section{Procédures concernant l'envoie des rapports au Parlement}

Au titre de l'Article $126 \mathrm{D}$ alinéa 1 de la Loi constitutionnelle fédérale, l'ACA établit un rapport sur ses constatations d'audit à l'intention du Conseil national. Plusieurs copies papier sont alors envoyées au Conseil national. Le rapport est discuté par la Commission de la Cour d'audit, réunie à cette occasion. Les MEP décident quels rapports seront débattus et à quel moment. Après que les MEP ont commenté les rapports, le Président de l'ACA expose les constatations d'audit. Le rapport est ensuite débattu en séance plénière du Conseil national, durant laquelle le Président de I'ACA peut prendre la parole après avoir entendu les commentaires des MEP.

Si nécessaire, I'ACA peut organiser des présentations d'information à l'intention des MEP avant les réunions de la Commission.

\section{Nombre et type de rapports}

L'ACA contrôle la gestion financière du secteur public en se concentrant sur les principes d'économie, d'efficience et d'efficacité. Étant inclusives, ses vérifications ne font pas de distinction entre les audits financiers, de conformité et de performance.

En 2015, I'ACA a présenté au Conseil national 18 compilations de rapports (71 rapports au total), le rapport sur les comptes des finances fédérales annuels, et son rapport annuel. Cette même année, 84 compilations de rapports (122 rapports au total) ont été soumises aux Länder et 8 compilations de rapports (8 rapports au total) aux municipalités.

En 2015, le Conseil national a traité les rapports de l'ACA lors de 10 réunions de la Commission de la Cour d'audit, 3 réunions de la Commission budgétaire et 5 séances plénières (ces séances peuvent également traiter des rapports qui n'ont pas été débattus les années précédentes). 


\section{3) Procédures parlementaires utilisées dans le traitement des rapports de l'ISC}

En vertu de l'Article $126 \mathrm{D}$ alinéa 2 de la Loi constitutionnelle fédérale, une commission permanente, la Commission de la Cour d'audit, a été instaurée par le Conseil national pour discuter les rapports de l'ACA. Les dispositions officielles régissant les commissions (formation, règles de procédure, etc.) sont décrites aux sections 29 à 45 de la Loi fédérale sur le Règlement intérieur du Conseil national. Le président du Conseil national organise la réunion, et la Commission élit un président, un secrétaire et, si nécessaire, des vice-présidents (section 34 alinéas 1 et 2 de la Loi fédérale sur le Règlement intérieur du Conseil national).

Le président élu de la Commission de la Cour d'audit est (régulièrement) un MEP de l'opposition. En début de réunion, la Commission nomme un rapporteur par rapport. Après le débat final en commission, la Commission recommande au Conseil national (en séance plénière) de prendre en compte le rapport d'audit débattu.

$\mathrm{Si}$ elles le jugent nécessaire, les commissions sont libres d'inviter des experts aux réunions des commissions. À titre d'exemple, pendant les deux dernières années, le membre autrichien de l'ECA a été invité deux fois aux réunions de la Commission de la Cour d'audit qui portaient sur des sujets européens.

\section{Personnel}

Six partis politiques sont représentés au Conseil national et à la Commission de la Cour d'audit. Les membres du Conseil national qui sont également membres de la Commission de la Cour d'audit sont secondés par un assistant par parti politique, qui est chargé de traiter les thèmes discutés par la Commission. La préparation des réunions est laissée aux soins des MEP et de leurs assistants.

\section{Participation de I'ISC à l'examen de ses rapports par le Parlement}

Le Président de l'ACA peut participer et prendre la parole aux séances plénières du Conseil national, et à ses commissions et sous-commissions réunies pour discuter des rapports de l'ACA et du rapport sur les comptes des finances fédérales. Cette procédure s'applique également aux discussions concernant les instructions d'investigation de problématiques et aux discussions sur le budget de l'ACA.

En vertu de la section 49 alinéa 1, I'ACA fait rapport au Conseil national de ses activités de l'année précédente et d'activités de contrôle spéciales, au titre de la Section 99, avant le 31 décembre de l'année. En outre, I'ACA peut à tout moment déposer devant le Conseil national des rapports contenant des constatations spécifiques.

Le Président doit soumettre les rapports de I'ACA pour délibération préliminaire à la commission permanente compétente (Commission de la Cour d'audit), à la séance suivant leur distribution. Les États des finances fédéraux sont soumis à la commission conformément à la section $32 \mathrm{~A}$.

La commission entame les discussions sur les rapports de l'ACA dans les 6 semaines qui suivent leur soumission. Elle choisit les personnes qui seront entendues à la réunion publique, sous réserve des dispositions de la section 28 B alinéa 2. Les enregistrements audio ou vidéo ne sont pas autorisés. Pendant le débat, toute contribution ne doit pas dépasser 10 minutes.

Le Président de I'ACA prend toujours part à ces réunions de la commission. Il est accompagné par le directeur général compétent, le chef de l'équipe d'audit et, si nécessaire, par un ou plusieurs membres de l'équipe d'audit. 


\section{4) Rapports de I'ISC}

L'ACA choisit le thème auquel elle va accorder la priorité dans son programme de contrôle de l'année à venir. Les systèmes de contrôle interne sont régulièrement un sujet d'audit. En 2014, le programme de contrôle de l'ACA s'est concentré sur les systèmes de contrôle internes, avec 20 audits réalisés dans ce domaine. Les résultats de ces audits sont intégrés dans les lignes directrices de l'ACA relatives aux systèmes de contrôle internes, ce qui renforce la fonction de conseil de l'ACA.

Tous les rapports publiés par l'ACA contiennent des conclusions claires et des opinions d'audit.

\section{5) Suivi des rapports de l'ISC}

L'ACA a mis en place un nouveau système d'évaluation de l'efficacité. En premier lieu, les recommandations contenues dans les rapports de l'année précédente sont passées en revue, à savoir que les organisations contrôlées sont interrogées sur l'avancement de la mise en œuvre des recommandations (contrôle administratif, classification : Réalisé / Assuré / En attente). Les résultats de l'enquête de suivi sont publiés dans le rapport d'activité annuel.

Suite à cette enquête de suivi, les audits effectués constituent la deuxième phase d'évaluation de l'efficacité. Les audits de suivi sont restreints, ils se concentrent sur la mise en œuvre de recommandations d'audit sélectionnées. L'ACA vérifie les progrès réellement accomplis par l'entité contrôlée (audit sur place). L'ACA publie un rapport séparé sur les conclusions de ces audits de suivi. Ces rapports sont à nouveau soumis à la procédure d'enquête annuelle.

Cette évaluation d'efficacité est un élément important pour la durabilité du contrôle car elle accroît la valeur des travaux de vérification et l'efficacité des recommandations.

\section{6) Audits réalisés à la demande du Parlement}

En vertu des Sections 1 alinéa 4 et 15 alinéa 4 de la Loi fédérale sur la Cour d'audit autrichienne, l'ACA peut être investie d'audits spéciaux par le Conseil national ou par une diète provinciale.

La Section 99 alinéa 1 du Règlement intérieur du Conseil national stipule que «1) Le Conseil national peut... décider de demander à la Cour d'audit de conduire des audits spéciaux sur la gestion des finances publiques. »

En vertu de la section 99 alinéa 2, ces audits peuvent également être demandés par une minorité qualifiée de 20 membres du Conseil national.

La Section 99 alinéa 3 ajoute qu'aucune motion supplémentaire ne peut être déposée tant que trois audits de gestion des finances publiques tels que définis à l'alinéa (2) sont en attente.

En 2015, I'ACA a mené 3 audits à la demande du Parlement (sur 100 au total).

\section{7) Programme de travail}

L'ACA prépare son programme de contrôle de manière indépendante. Le Parlement est habilité à demander des audits spéciaux (voir ci-dessus). 


\section{8) Niveau et fréquence des échanges}

L'ACA offre des informations complètes aux questions posées par les membres du Conseil national et leur personnel sur les audits qu'elle a publiés. Cette activité donne lieu à des échanges actifs avec les membres du Conseil national et leurs employés, grâce auxquels I'ACA actualise régulièrement ses connaissances sur les changements et les tendances au Conseil national. L'objectif consiste à entretenir des contacts constants avec les membres du Conseil national.

Le service de la communication et des relations avec le Parlement de l'ACA est responsable de ces contacts réguliers.

Aux réunions de la Commission de la Cour d'audit, des échanges officiels ont lieu une fois par mois (une dizaine de réunions par an).

\section{9) Bonnes pratiques}

En ce qui concerne ses contrôles des comptes et de la gestion financière, le Conseil national s'appuie sur les audits réalisés par I'ACA, laquelle tient de nombreuses réunions dans ce but. Comme il l'a été mentionné ci-dessus, la Commission de la Cour d'audit a tenu 10 réunions en 2015, ce qui a donné lieu à des discussions soutenues sur les résultats des audits de l'ACA. 


\section{BELGIQUE}

Cour des comptes

\section{1) Cadre juridique}

Établie par l'Article 180 de la Constitution, la Cour des comptes belge est un organe collatéral du Parlement. Elle examine d'un point de vue externe les opérations budgétaires, comptables et financières de l'État fédéral, des communautés, des régions, des organismes publics qui en dépendent, et des provinces.

Les pouvoirs de la Cour sont définis par la loi du 29 octobre 1846, amendée depuis plusieurs fois, et par la loi du 16 mai 2003. La loi accorde une grande indépendance à la Cour et une véritable autonomie dans l'exercice de ses fonctions.

Les règles de procédure, ont été votées par la Chambre des représentants le 5 février 1998 et publiées au Journal officiel belge le 4 septembre 1998.

L'Article 5, paragraphe 1, alinéa 4 de la loi du 29 octobre 1846 relative à l'organisation de la Cour des comptes dispose: " La Chambre des représentants peut charger la Cour des comptes de procéder, au sein des services et organismes soumis au contrôle de la Cour, à un contrôle de légalité et de régularité de certains programmes de dépenses ainsi qu'à des audits financiers. "

Le dernier alinéa de l'Article 5, paragraphe 1, de la loi du 29 octobre 1846, habilite la Chambre des représentants à charger la Cour des comptes de procéder, au sein des services et organismes soumis à son contrôle, à des analyses de gestion.

Cette disposition juridique a été récemment appliquée.

2) Modalités de soumission des rapports de l'ISC au parlement

\section{Obligations juridiques de I'ISC sur la publication de rapports}

II convient de se référer par exemple à l'Article 180 de la Constitution belge. Voir https://www.dekamer.be/kvvcr/pdf sections/publications/constitution/GrondwetUK.pdf.

Voir également la Loi fédérale du 22 mai 2003 (en français uniquement) : Loi du 22 mai 2003 portant organisation du budget et de la comptabilité de l'État fédéral :

Art. 54 . Le cas échéant, la Cour des comptes communique à la Chambre des représentants ses remarques sur les documents visés aux articles 45, 50, 51, 52 en 53.

Art. 75. Avant le [31 octobre] de l'année qui suit l'année budgétaire, la Cour des comptes transmet le compte général de l'administration générale à la Chambre des représentants avec ses observations, http://www.begroting.be/FR/law/loi\%20du\%2022\%20mai\%202003.htm. 


\section{Objectifs de la politique de communication}

La politique de communication reflète les valeurs de la Cour des comptes ainsi que les principes de communication présentés dans sa déclaration de mission.

Cette politique a pour but de diffuser une information exacte, objective, complète, actuelle, claire et utile sur les contrôles exercés par la Cour des comptes. Ces informations sont communiquées sous forme de rapports en version papier et électronique. La Cour des comptes fournit directement aux assemblées parlementaires des informations utiles et fiables, résultant de ses travaux. Les constatations, les opinions et les recommandations formulées dans les rapports permettent à ce destinataire privilégié d'exercer sa mission législative et budgétaire, ainsi que de contrôler le pouvoir exécutif. Grâce à la large diffusion de ses rapports clairement rédigés, la Cour des comptes contribue dans une large mesure à l'amélioration de la gestion des administrations publiques.

La déclaration relative à la politique de communication de la Cour des comptes détermine :

- les principes sous-jacents à la politique de communication de la Cour,

- le caractère public de ses rapports,

- les produits issus de son activité et les modalités de mise en œuvre de cette politique.

\section{Principes de la politique de communication}

- La politique de communication vise à assurer une présentation claire et univoque des constations, des conclusions et de recommandations apportées par la Cour des comptes suite à ses investigations.

- Dans un souci d'offrir une information de qualité, les discussions menées avec l'administration et le ministre compétent sont consignées dans les publications de la Cour des comptes.

- La Cour des comptes envoie puis, si nécessaire, présente ses rapports aux assemblées parlementaires, au sein desquelles ils sont discutés.

- Appliquant les normes internationales d'audit externe et plus précisément le principe de la procédure de vérification, la Cour des comptes fournit uniquement les informations contenues dans les rapports publiés. Ses travaux clarifient ultérieurement le champ d'application de ses rapports et ils viennent corriger les interprétations éventuellement erronées.

- Les rapports de la Cour des comptes sont publiés dans les langues des assemblées parlementaires concernées.

- Dans son processus de communication, la Cour des comptes s'assure que la confidentialité des données est préservée.

\section{Publicité des rapports de la Cour des comptes}

La politique de communication de la Cour des comptes s'appuie sur le caractère public de ses rapports. Toutefois, les assemblées parlementaires sont les premières instances informées des rapports, avant les médias et le grand public. 
Cette politique concerne :

- les informations destinées aux représentants parlementaires,

- les informations destinées aux ministres et aux autorités publiques concernées,

- les relations avec la presse,

- la transmission des rapports de la Cour à toute personne ou organisme intéressé.

\section{Produits et mise en pratique de la politique de communication}

Les publications de la Cour des comptes comprennent un rapport annuel sur les comptes fédéraux, des Communautés et des Régions présentant le résultat des contrôles exercés sur ces comptes, et des rapports spécifiques réalisés en vertu de dispositions particulières ou lorsque les résultats des contrôles attestent de la nécessité d'informer l'assemblée parlementaire. La Cour des comptes publie également un rapport d'activité annuel. Il est possible de télécharger gratuitement ces publications à partir de son site Internet, ainsi que certaines données, comptes ou résultats de contrôles disponibles uniquement au format numérique et qui ne sont pas consignés dans les rapports annuels ou dans des rapports séparés.

Ce site Internet est la source d'informations la plus complète et la plus actuelle sur les rapports publiés par la Cour.

La Cour des comptes publie des communiqués de presse et organise des conférences de presse pour exprimer son point de vue et répondre aux questions des journalistes.

\section{Discussions avec la commission parlementaire}

Des représentants de la Cour présentent les résultats des contrôles à la commission parlementaire. Les parlementaires qui la composent peuvent poser des questions.

\section{Rapport d'activité}

Autrefois, le rapport d'activité annuel n'était pas publié. Les données de 2015 seront intégrées dans le rapport d'activité annuel de 2015 que la Cour prévoit de publier rapidement sur https://www.ccrek.be/EN/Publications/AnnualReports.html.

\section{3) Procédures parlementaires utilisées dans le traitement des rapports de I'ISC}

La procédure instaure des auditions au cours desquelles l'entité contrôlée ou d'autres personnes appropriées sont invitées à apporter des clarifications ou des informations complémentaires.

Le débat sur les rapports de I'ISC se tient au sein d'une (des) commission(s).

Le document parlementaire en résultant résume le débat qui s'est tenu sur le rapport de l'ISC.

Le parlement discute et adopte la loi relative au compte générale en octroyant la décharge au gouvernement. 


\section{Personnel}

Chaque parlement sélectionne de manière souveraine la commission parlementaire chargée des rapports de l'ISC ainsi que les procédures à suivre.

Les documents parlementaires résultant du débat sur un rapport de I'ISC sont rédigés par le personnel parlementaire rattaché à la (aux) commission(s).

\section{Participation de I'ISC à l'examen de ses rapports par le Parlement}

Des représentants de la Cour (membre, directeur, superviseur et auditeur(s)) présentent les résultats des contrôles à la commission parlementaire compétente. Les parlementaires peuvent poser des questions.

\section{4) Rapports de l'ISC}

La Cour formule des recommandations à l'entité contrôlée dans des rapports d'audit (voir la déclaration de mission, en français uniquement : http://www.ccrek.be/FR/DeclarationDeMission.htm).

La configuration et le fonctionnement des systèmes de contrôle internes peuvent être un thème mis en évidence dans les rapports de I'ISC.

Tous les rapports d'audit contiennent des conclusions et des opinions d'audit claires.

L'un des principaux objectifs de la Cour des comptes belge consiste à instaurer une communication claire avec les parties prenantes. À ces fins, elle emploie une équipe d'experts en communication dont le rôle est d'assister les contrôleurs dans la rédaction de leurs rapports.

Voir : Ardelean, Veronica, European Court of Auditors, Clear language event at the European Court of Auditors, Journal of the European Court of Auditors, December 2015, p. 27, http://www.eca.europa.eu/Lists/ECADocuments/JOURNAL15 12/QJ-AD-15-0102A-C.pdf.

\section{5) Suivi des rapports de l'ISC}

\section{Projets de budget}

Les rapports de I'ISC sont périodiquement utilisés comme source d'informations dans le débat annuel sur les projets de budget.

Voir https://www.ccrek.be/EN/Presentation/Competences.htm|\#BudgetAnalysis.

\section{Suivi des rapports de l'ISC}

La Cour des comptes assure régulièrement le suivi de ses contrôles pour veiller à ce que les recommandations publiées aient été prises en compte par les entités contrôlées. Chaque rapport d'audit financier commence par une section qui reprend le niveau de mise en œuvre des contrôles réalisés l'année précédente.

Chaque Parlement décide d'une façon souveraine de la suite qui sera donnée aux recommandations de la Cour des comptes. 
À titre d'exemple, le protocole d'accord passé entre le Parlement des Flandres et la Cour des comptes belge le 20 mars 2012 (voir l'Article 74 des Règles de procédure du Parlement flamand) stipule que la Cour devra dresser un inventaire, structuré selon les lettres de mission des différents ministres, des recommandations auxquelles le gouvernement flamand est supposé répondre. Cet inventaire dans son ensemble est envoyé au Président du Parlement flamand, et tous les ministres reçoivent simultanément la partie de l'inventaire qui contient les recommandations qu'ils ont commentées dans leur lettre de mission. Il est prévu que les ministres établissent un rapport décrivant la manière dont les recommandations ont été mises en œuvre.

\section{Audits réalisés à la demande du Parlement}

L'Article 5, paragraphe1, 4 de la loi du 29 octobre 1846 organisant la Cour des comptes dispose : "La Chambre des représentants peut charger la Cour de procéder, au sein des services et organismes soumis au contrôle de la Cour, à un contrôle de légalité et de régularité de certains programmes de dépenses, ainsi qu'à des audits financiers. »

Le dernier alinéa de l'Article 5, paragraphe1, de la loi du 29 octobre 1846 dispose que la Chambre des représentants peut charger la Cour de procéder, au sein des services et organismes soumis à son contrôle, à des analyses de gestion.

Cette disposition juridique a été récemment appliquée.

\section{6) Programme de travail}

L'ISC ne consulte pas le Parlement sur son programme de travail annuel en matière de contrôles.

Il n'existe pas de consultation officielle avec le Parlement pour définir le programme de travail. Toutefois, lors du processus de détermination du programme opérationnel annuel et des thèmes de contrôles la Cour tient compte des préoccupations exprimées par les assemblées législatives dans leurs débats et à l'occasion de leurs autres activités (questions, résolutions, initiatives législatives).

\section{7) Niveau et fréquence des échanges}

II y a des échanges réguliers (y compris des échanges informels) entre la Présidence de la Cour des comptes et le Président du Parlement.

\section{8) Bonnes pratiques}

L'un des principaux objectifs de la Cour des comptes belge consiste à instaurer une communication claire avec les parties prenantes. À ces fins, la Cour des comptes belge emploie une équipe d'experts en communication dont le rôle est d'assister les contrôleurs dans la rédaction de leurs rapports.

Voir : Ardelean, Veronica, European Court of Auditors, Clear language event at the European Court of Auditors, Journal of the European Court of Auditors, December 2015, p. 27, http://www.eca.europa.eu/Lists/ECADocuments/JOURNAL15 12/QJ-AD-15-0102A-C.pdf. 


\section{BOSNIE-HERZÉGOVINE}

Institution supérieure de contrôle

\section{1) Cadre juridique}

Loi sur les contrôles, Article 12 (Planification des contrôles) : L'Auditeur général adopte, après avoir consulté son ou ses adjoints, le plan de contrôle annuel du Bureau des audits pour l'année à venir, en prenant en compte les exigences définies dans cette loi. Le plan de contrôle sera soumis pour information à la commission parlementaire, avant le début du nouvel exercice financier.

Loi sur les contrôles, Article 16 (Rapports de contrôle) : Le Bureau des audits soumet un rapport d'audit financier à l'entité contrôlée (dans les 90 jours suivant la soumission du rapport annuel de l'entité contrôlée) et au Parlement.

Concernant l'audit du rapport annuel sur l'exécution budgétaire tel que stipulé à l'Article 13, alinéa (5) de cette loi, le Bureau des audits soumet le rapport d'audit au Parlement dans les 90 jours suivant la réception du rapport annuel sur l'exécution du budget.

Le Bureau des audits soumet à l'entité contrôlée et au Parlement un rapport d'audit de performance ou un rapport d'audit spécial dans les 30 jours suivant l'achèvement de l'audit.

Le Bureau des audits regroupe dans son rapport d'audit annuel au Parlement (rapport d'audit résumé) les constatations et les recommandations essentielles issues des audits financiers, des audits de performance et des audits spéciaux réalisés. Le rapport d'audit annuel remis au Parlement doit être soumis avec le rapport de l'audit exécuté sur le rapport annuel sur l'exécution du budget, ce dernier faisant partie intégrante du rapport annuel.

Loi sur les contrôles, Article 17 (autre rapport) : le Bureau des audits peut, au moment qu'il juge nécessaire, soumettre au Parlement un autre rapport d'audit portant sur des problématiques pertinentes.

Loi sur les contrôles, Article 18 (audits spéciaux) : Le Parlement ou une commission parlementaire peut à tout moment demander au Bureau des audits de mener un audit spécial. Le Bureau des audits décidera s'il y a lieu d'exécuter un audit spécial tel que décrit à l'alinéa (1) du présent Article, et en notifiera par écrit le Parlement, conformément à l'Article 16 de la présente Loi.

\section{2) Modalités de soumission des rapports de l'ISC au parlement}

Le Bureau des audits soumet au Parlement des rapports d'audit financiers (audit de conformité inclus), des rapports d'audit de performance, des rapports spéciaux, un rapport annuel sur les constatations et les recommandations essentielles (rapport de synthèse) et un rapport d'audit annuel sur l'exécution du budget de l'État. Ces rapports sont adressés aux deux chambres et à leurs commissions parlementaires compétentes.

Le Bureau des audits soumet son rapport financier sur une base trimestrielle et annuelle, avec son rapport d'activité annuel. Ces rapports sont adressés au président de la commission parlementaire compétente. 
Le Bureau des audits soumet également au Parlement des rapports sur son évaluation professionnelle (rapport d'évaluation par les pairs), incluant le compte-rendu des mesures que le Bureau des audits a prises en réponse à cette évaluation. Ce rapport est adressé au président de la commission parlementaire compétente.

\section{Procédures d'envoie des rapports au Parlement}

Le Bureau des audits ne dispose pas de procédures spécifiques écrites. Pendant le débat portant sur les rapports d'audit, l'Auditeur général présente les constatations essentielles contenues dans les rapports d'audit devant la commission parlementaire.

Nombre et type de rapports

\begin{tabular}{|c|c|c|c|c|c|c|c|}
\hline & $\begin{array}{l}\text { Rapports } \\
\text { d'audit } \\
\text { financier }\end{array}$ & \begin{tabular}{|c|} 
Rapports d'audit \\
de conformité
\end{tabular} & $\begin{array}{l}\text { Rapports d'audit } \\
\text { de performance }\end{array}$ & \begin{tabular}{|c|} 
Autres rapports \\
ou documents
\end{tabular} & $\begin{array}{l}\text { Rapports } \\
\text { d'activité } \\
\text { annuels }\end{array}$ & \begin{tabular}{|c|} 
Rapports \\
demandés par \\
le Parlement
\end{tabular} & Remarques \\
\hline $\begin{array}{c}\text { Nombre de } \\
\text { rapports soumis }\end{array}$ & 73 & & 4 & & 1 & 1 & $\begin{array}{l}\text { L'audit de } \\
\text { conformité } \\
\text { est intégré à } \\
\text { l'audit } \\
\text { financier }\end{array}$ \\
\hline $\begin{array}{c}\text { Nombre de } \\
\text { rapports discutés } \\
\text { par le Parlement }\end{array}$ & 73 & & 4 & & 1 & 1 & \\
\hline $\begin{array}{l}\text { Soumis au } \\
\text { Président }\end{array}$ & 0 & & 0 & & $\mathrm{~N}$ & 0 & \\
\hline $\begin{array}{l}\text { Soumis au } \\
\text { Président de la } \\
\text { commission } \\
\text { spécialisée }\end{array}$ & 0 & & 0 & & 0 & 0 & \\
\hline $\begin{array}{l}\text { Copies envoyées } \\
\text { aux autres } \\
\text { commissions } \\
\text { concernées }\end{array}$ & $\mathrm{N}$ & & $\bar{N}$ & & $\mathrm{~N}$ & $\bar{N}$ & \\
\hline $\begin{array}{c}\text { Notes de synthèse } \\
\text { destinées à la } \\
\text { commission } \\
\text { parlementaire }\end{array}$ & 0 & & 0 & & $\mathrm{~N}$ & 0 & \\
\hline $\begin{array}{c}\text { Résumés soumis au } \\
\text { Parlement }\end{array}$ & 0 & & 0 & & $\mathrm{~N}$ & 0 & \\
\hline
\end{tabular}




\section{3) Procédures parlementaires utilisées dans le traitement des rapports de l'ISC}

Des procédures établies visant le traitement des rapports de I'ISC sont définies dans les lignes directrices du Parlement pour l'examen et l'analyse des rapports d'audit. Ces procédures ne concernent que les rapports d'audit financiers. Ce type de procédures ne s'applique pas aux rapports d'audit de performance.

Les deux chambres parlementaires disposent d'une commission spécialisée (Commission du budget et des finances) affectée aux rapports de I'ISC. Elle est présidée par un membre du ou des partis au gouvernement (en fonction de la procédure).

Cette commission désigne un rapporteur pour un rapport d'audit donné.

Des auditions ont lieu auxquelles est conviée l'entité contrôlée ou d'autres personnes appropriées afin d'apporter des clarifications ou un complément d'informations.

Le débat sur les rapports de l'ISC se déroule au sein d'une (des) commission(s) et en séance plénière.

Des commissions autres que les commissions compétentes analysent individuellement les rapports de I'ISC si ces derniers abordent les problématiques soumises à leur contrôle.

Un rapport réunissant les conclusions du débat parlementaire sur un rapport de l'ISC est publié sur le site Internet du Parlement.

Le Parlement dispose d'une procédure de décharge établie concrètement appliquée. Les rapports de l'ISC jouent un rôle dans cette procédure.

\section{Personnel}

Les deux chambres analysent les rapports de I'ISC par le biais de la Commission du budget et des finances. Les membres de cette commission sont assistés par le secrétaire de commission, et par un ou deux assistants administratifs. Le secrétaire est chargé de rédiger les notes d'information, les analyses et les questions, ainsi que les conclusions préliminaires, résultat de l'examen des rapports de I'ISC par la Commission.

\section{Participation de l'ISC à l'examen de ses rapports par le Parlement}

L'Auditeur général (accompagné d'autres membres du personnel) assiste aux réunions parlementaires lorsque les rapports de I'ISC sont débattus; il présente les constatations et les recommandations essentielles contenues dans les rapports d'audit débattus. Le personnel de I'ISC est également disponible pour apporter toute clarification nécessaire au débat.

\section{3) Rapports de I'ISC}

L'ISC rédige un rapport annuel à l'intention du Parlement; ce rapport répertorie tous les problèmes systémiques découverts pendant les contrôles. II propose des mesures visant à renforcer les systèmes et à améliorer la transparence et l'efficience des deniers publics. Les rapports d'audit financier en particulier traitent des systèmes de contrôle internes des entités contrôlées, avec une section dédiée au contrôle interne. Tous les rapports d'audit financier offrent une opinion claire sur les états financiers et la conformité, et tous les rapports d'audit de performance contiennent des conclusions claires. 


\section{4) Suivi des rapports de I'ISC}

L'ISC conserve dans un fichier Excel une trace des conclusions et des recommandations transmises à l'entité contrôlée, ainsi que leur niveau de mise en œuvre ; ce fichier est régulièrement mis à jour.

Le Parlement émet périodiquement des conclusions à l'intention des entités contrôlées pour qu'elles mettent en œuvre les recommandations de I'ISC, mais il n'y a pas de mécanisme lui permettant de suivre la mise en œuvre de ces conclusions. C'est pourquoi le niveau de réalisation des recommandations de I'ISC est relativement bas (50-60\%).

Les audits financiers comportent deux étapes. L'étape de vérification provisoire fait le bilan de la mise en œuvre des recommandations issues des audits précédents. Les mesures prises en réponse à ces recommandations sont analysées. Les rapports des audits financiers définitifs comportent tous une section séparée qui analyse le niveau de mise en œuvre des recommandations concernant l'année précédente. Cette section se divise habituellement en trois sous-sections : les recommandations mises en œuvre, les recommandations en cours de réalisation et les recommandations non mises en œuvre. Cette approche intègre également la mise en œuvre des recommandations liées au système de contrôle interne.

Les entités contrôlées font rapport à l'Auditeur général et au ministère des Finances dans les 60 jours suivant la réception du rapport d'audit, en précisant les actions prises pour venir à bout des faiblesses, des irrégularités et des non-conformité identifiées dans le rapport d'audit.

En s'appuyant sur les constatations et les recommandations contenues dans le rapport d'audit annuel et / ou le rapport annuel sur l'exécution budgétaire, le Parlement est habilité à réduire le budget d'un ou plusieurs utilisateurs ou de prendre des actions correctives. Les rapports de I'ISC servent très souvent de base au débat sur les projets de budget, notamment pour les entités contrôlées avec des opinions avec réserves.

\section{5) Audits réalisés à la demande du Parlement}

La disposition juridique figure à I'Article 18 (voir la réponse 1). L'ISC a le droit discrétionnaire de réaliser ou non un contrôle spécial demandé par le Parlement. Ce dernier n'a pas très souvent recours à cette disposition. Le Parlement n'a demandé qu'un seul audit durant les cinq dernières années. L'ISC n'a jamais refusé d'exécuter un audit demandé par le Parlement.

\section{6) Programme de travail}

Après consultation de ses adjoints, l'Auditeur général adopte le plan de travail annuel en tenant compte des exigences de la Loi (par exemple des audits demandés par le Parlement). Le plan de contrôle est soumis à la commission parlementaire à titre d'information avant le début du nouvel exercice financier.

\section{7) Niveau et fréquence des échanges}

Dans le cadre du travail, des échanges ont lieu régulièrement entre le personnel de l'ISC concerné (il n'y a pas d'unité spécifiquement chargée des relations parlementaires) et le personnel administratif des commissions parlementaires compétentes. La fréquence de ces contacts dépend des besoins de l'ISC et du Parlement. 


\section{8) Bonnes pratiques}

Les procédures écrites encadrant l'examen des rapports d'audit financiers ont incontestablement accru l'efficacité de l'examen parlementaire des rapports d'audit ainsi que la compréhension des contrôles.

\section{9) Perspective}

Il manque des procédures écrites destinées à l'examen des rapports d'audit de performance.

Le manque de mécanisme parlementaire dédié au suivi de la mise en œuvre des recommandations d'audit par les entités contrôlées réduit l'influence des travaux de I'ISC ainsi que l'efficience de ses rapports.

Des projets sont à l'étude pour rédiger des lignes directrices destinées à l'examen des rapports d'audit de performance afin de renforcer l'impact de ces rapports et d'améliorer leur compréhension par le Parlement.

Ces activités devraient et doivent inclure la mise en œuvre des recommandations de I'ISC, avec des procédures parlementaires de suivi de ces réalisations, et de rendre les entités contrôlées responsables de leurs manquements à cet égard.

Par ailleurs, il existe un besoin de former régulièrement les parlementaires aux principes du contrôle du secteur public, notamment dans la perspective du mandat parlementaire de quatre ans.

10) Y-a-t-il d'autres éléments concernant les relations actuelles entre I'ISC et le Parlement que vous souhaiteriez souligner, et quelles a été leur évolution au cours des cinq dernières années?

Nous souhaiterions évoquer la question de l'indépendance de I'ISC. Le Parlement devrait avoir la formation adéquate afin de ne pas voter des lois et des réglementations qui minent l'indépendance du Bureau des audits ; ils devraient être par ailleurs davantage sensibilisés à l'importance d'avoir une ISC indépendante. 


\section{BULGARIE}

Bureau d'audit national

\section{1) Cadre juridique}

Aux termes de la Constitution de la République de Bulgarie, l'Assemblée nationale instaure une Institution supérieure de contrôle pour superviser l'exécution du budget.

L'organisation, les pouvoirs et les procédures du Bureau d'audit national (BNAO) sont définis par la Loi relative au Bureau d'audit national.

Les obligations du BNAO envers le Parlement sont énoncées dans le chapitre de la Loi qui porte sur la responsabilité et le contrôle de ses fonctions.

Le BNAO présente au Parlement les rapports suivants :

1. Rapports contenant des opinions sur les déclarations d'exécution du budget de l'État, du budget du régime de sécurité sociale publique, du budget du fonds d'assurance-maladie national et des dépenses budgétaires de la Banque nationale de Bulgarie (BNB) pour l'année précédente.

2. Rapports relatifs aux contrôles réalisés sur des mécanismes budgétaires ou aux contrôles dont les résultats présentent un intérêt dans le cadre des budgets respectifs et des ressources et activités publiques diverses.

3. Rapports relatifs aux contrôles exécutés sur décision de l'Assemblée nationale.

L'Assemblée nationale ou l'une de ses commissions peut demander au BNAO de soumettre des rapports d'audit particuliers.

Le BNAO peut faire des propositions à l'Assemblée nationale et à ses commissions spécialisées afin d'examiner des rapports d'audit qui sont particulièrement importants quant à l'amélioration de la discipline budgétaire et de l'administration du budget et / ou d'autres fonds et activités publics.

Au plus tard le 30 septembre, le BNAO soumet à l'Assemblée nationale un rapport d'activité concernant l'année précédente.

L'état financier annuel du BNAO est vérifié par une commission indépendante comprenant au moins deux contrôleurs certifiés. Le nombre de membres et la composition de la commission sont déterminés par I'Assemblée nationale. Le rapport de la commission est soumis à l'Assemblée nationale, accompagné du rapport d'activité du BNAO de l'année correspondante. Le Président du BNAO peut exprimer une opinion écrite sur le rapport, et cette opinion est jointe au rapport soumis à l'Assemblée nationale. Le rapport et I'opinion du Président du BNAO sont rendus publics après adoption par l'Assemblée nationale.

\section{2) Modalités de soumission des rapports de l'ISC au parlement}

Le cadre général dans lequel s'inscrit l'obligation de soumettre des rapports est décrit dans la section précédente. 
Un rapport spécial faisant état des constatations et des recommandations résultant des audits de performance réalisés sur la gestion des fonds de l'Union européenne est soumis à la commission pour les Affaires européennes et la supervision des fonds européens.

Si le BNAO découvre des erreurs et des inexactitudes qui ont une incidence substantielle sur les indicateurs concernant l'exécution du budget de l'État, il doit notifier les corrections à apporter au rapport au ministre des Finances et à la commission de l'Assemblée nationale chargée du budget.

En vertu de la Loi sur la gestion et le contrôle des finances du secteur public, le BNAO soumet à l'Assemblée nationale un rapport sur la situation des systèmes de contrôle et de gestion des finances, ainsi que l'audit interne et les opinions sur les rapports, au plus tard le 30 juin.

\section{Procédures régissant l'envoie des rapports au Parlement}

L'envoi des rapports au Parlement n'est régi par aucune procédure spécifique.

Nombre et type de rapports

\begin{tabular}{|c|c|c|c|c|c|c|c|}
\hline & $\begin{array}{l}\text { Rapports } \\
\text { d'audit } \\
\text { financier }\end{array}$ & $\begin{array}{l}\text { Rapports d'audit } \\
\text { de conformité }\end{array}$ & $\begin{array}{l}\text { Rapports d'audit } \\
\text { de performance }\end{array}$ & $\begin{array}{c}\text { Autres rapports } \\
\text { ou documents }\end{array}$ & $\begin{array}{l}\text { Rapports } \\
\text { d'activité } \\
\text { annuels }\end{array}$ & $\begin{array}{c}\text { Rapports } \\
\text { demandés par } \\
\text { le Parlement }\end{array}$ & Remarques \\
\hline $\begin{array}{c}\text { Nombre de } \\
\text { rapports soumis }\end{array}$ & 1 & 3 & 10 & 1 & 1 & 1 & \\
\hline $\begin{array}{l}\text { Nombre de } \\
\text { rapports discutés } \\
\text { par le Parlement }\end{array}$ & & & 1 & & & & \\
\hline $\begin{array}{l}\text { Soumis au } \\
\text { Président }\end{array}$ & $\mathrm{N}$ & $N$ & $\mathrm{~N}$ & $\mathrm{~N}$ & $N$ & $\mathrm{~N}$ & \\
\hline $\begin{array}{l}\text { Soumis au } \\
\text { Président de la } \\
\text { commission } \\
\text { spécialisée }\end{array}$ & $\mathrm{O}$ & $\mathrm{O}$ & 0 & 0 & 0 & 0 & \\
\hline $\begin{array}{c}\text { Copies envoyées } \\
\text { aux autres } \\
\text { commissions } \\
\text { concernées }\end{array}$ & 0 & $\mathrm{O}$ & 0 & 0 & 0 & 0 & \\
\hline $\begin{array}{c}\text { Notes de synthèse } \\
\text { destinées à la } \\
\text { commission } \\
\text { parlementaire }\end{array}$ & $\mathrm{N}$ & $\mathrm{N}$ & $N$ & $\mathrm{~N}$ & $\mathrm{~N}$ & $\mathrm{~N}$ & \\
\hline $\begin{array}{c}\text { Résumés soumis au } \\
\text { Parlement }\end{array}$ & $\mathrm{N}$ & $\mathrm{N}$ & $\mathrm{N}$ & $\mathrm{N}$ & $\mathrm{N}$ & $\mathrm{N}$ & \\
\hline
\end{tabular}

3) Procédures parlementaires utilisées dans le traitement des rapports de I'ISC 
Un débat sur les rapports soumis par l'ISC a lieu au sein des commissions parlementaires compétentes.

Une sous-commission de la Commission du budget et des finances (sous-commission chargée de la responsabilité du secteur public) exerce le contrôle parlementaire, mais il n'existe pas de procédures établies encadrant le traitement des rapports du BNAO.

\section{Personnel}

En règle générale, dans l'exercice de leurs fonctions, les membres des commissions permanentes sont assistés par du personnel administratif, mais ce personnel n'est pas spécifiquement affecté aux rapports de I'ISC.

\section{Participation de I'ISC à l'examen de ses rapports par le Parlement}

Lorsque la commission parlementaire discute des rapports d'audit, la Direction et le chef de l'équipe d'audit du BNAO assistent aux réunions. Ils présentent les principales constatations, conclusions et recommandations de l'audit, et ils répondent aux questions.

\section{4) Rapports de I'ISC}

Les principales constatations, conclusions et recommandations des audits sont présentées au cours des débats de la commission permanente.

La configuration et le fonctionnement des systèmes de contrôle internes sont vérifiés lors des audits de performance et de conformité.

Les rapports d'audit de performance et de conformité contiennent des conclusions claires, tandis que les rapports d'audit financier contiennent les opinions de l'audit.

\section{5) Suivi des rapports de I'ISC}

Le suivi des recommandations est encadré par les règles relatives à l'organisation et au contrôle de la mise en œuvre des recommandations du BNAO.

Le contrôle de la mise en œuvre des recommandations est une étape obligatoire de tout audit qui contient des recommandations.

Les travaux de suivi ont pour but de vérifier les actions prises par les dirigeants des entités contrôlées aux fins de mettre en œuvre les recommandations du BNAO. Ce suivi englobe un bilan systématique, une analyse des recommandations dans différents domaines et une évaluation des activités destinées à améliorer la gestion budgétaire et de divers fonds et activités publics.

Si des recommandations ne sont pas mises en œuvre, les organes concernés en sont informés sans délais.

Un registre électronique permet d'assurer le suivi des recommandations.

Si une recommandation n'est pas mise en œuvre, le BNAO rédige un rapport contenant des propositions à l'intention de l'Assemblée nationale, du Conseil des ministres ou du conseil municipal concerné afin de prendre les actions qui s'imposent.

Tout manquement à la mise en œuvre des recommandations est annoncé sur le site Internet du BNAO. 


\section{6) Audits réalisés à la demande du Parlement}

En plus des audits prévus au programme annuel, l'Assemblée nationale peut demander au BNAO d'exécuter jusqu'à 5 contrôles par an.

En 2015, l'Assemblée nationale a demandé un audit sur la supervision bancaire après que l'attention du public a été attirée par la situation difficile d'une banque. En vertu de la décision de l'Assemblée nationale, le BNAO a exécuté un audit de performance sur l'efficacité et l'efficience de la supervision bancaire par la Banque nationale bulgare pour la période allant du $1^{\mathrm{er}}$ janvier 2012 au 31 décembre 2014.

\section{7) Programme de travail}

Le programme de contrôle annuel est adopté par le BNAO sans consulter l'Assemblée nationale.

Le programme de contrôle annuel est soumis à l'Assemblée nationale dans les 7 jours suivant son adoption ou son amendement.

En plus des audits prévus au programme annuel, I'Assemblée nationale peut demander au BNAO d'exécuter jusqu'à 5 contrôles par an.

\section{8) Niveau et fréquence des échanges}

Le dirigeant du BNAO a un échange mensuel avec le Président de la Commission du budget et des finances et avec la sous-commission chargée de la responsabilité du secteur public.

Le Chef de cabinet du Président du BNAO est chargé des relations avec le Parlement.

\section{9) Bonnes pratiques}

Suite au contrôle effectué par le BNAO sur l'organisation et la gestion du processus budgétaire par le ministère des Finances, une nouvelle loi sur les finances publiques a été adoptée par l'Assemblée nationale.

\section{0) Perspective}

L'Assemblée nationale devrait examiner les rapports formulant des opinions sur les déclarations d'exécution du budget de l'État, du budget du régime de la sécurité sociale publique, du budget du fonds de l'assurance-maladie nationale, des dépenses budgétaires de la BNB, ainsi que les rapports liés aux audits exercés sur décision de l'Assemblée nationale dans les trois mois suivant leur date de soumission.

En règle générale, la période définie n'est pas respectée et certains rapports soumis ne sont pas examinés.

Le BNAO va s'employer à mettre en place des mécanismes obligeant à prendre en compte les rapports formulant des opinions sur les déclarations d'exécution du budget de l'État, du budget du régime de la sécurité sociale publique et du budget du fonds de l'assurance-maladie nationale avant l'adoption des différents budgets des années suivantes par l'Assemblée nationale. 
Un autre objectif vise à établir une coopération entre le nouveau Conseil budgétaire et le BNAO sur les opinions concernant l'exécution du budget de l'État et l'exécution des budgets des institutions autonomes, comme le Fonds de l'assurance-maladie nationale. 


\section{CROATIE}

Bureau national d'audit

\section{1) Cadre juridique}

Le cadre juridique qui régit les relations entre le Bureau national d'audit croate (SAO) et le Parlement croate est énoncé dans la Loi relative au Bureau national d'audit (OG 80/11), la Loi sur le financement des campagnes électorales et de l'activité politique (OG 24/11,61/11, 27/13 i 48/13 - texte révisé) et le Règlement intérieur du Parlement croate.

\section{2) Modalités de soumission des rapports de l'ISC au parlement}

Le SAO remet au Parlement croate un rapport de travail annuel ainsi que d'autres rapports d'audit (individuellement ou groupés). Tous les rapports sont adressés au Président du Parlement.

Le délai de soumission du rapport de travail annuel du SAO est défini à l'Article 15 de la Loi sur le Bureau national d'audit. L'Auditeur général rend compte du travail du SAO auprès du Parlement croate à la fin de l'année pour l'exercice commençant le $1^{\mathrm{er}}$ octobre de l'année précédente et se terminant le 30 septembre de l'année en cours. Outre le rapport annuel, le SAO soumet des rapports liés aux contrôles menés durant cet exercice.

L'obligation de soumettre au Parlement le rapport d'audit sur l'exécution du budget de l'État est également définie par la Loi sur le Bureau national d'audit, avant le $1^{\mathrm{er}}$ juin de l'année courante pour le budget de l'année précédente.

Les rapports concernant le contrôle des partis politiques, des représentants indépendants et des membres indépendants de collectivités locales sont soumis au Parlement croate avant la fin de l'année en cours pour les activités de l'année précédente, conformément à la Loi relativement aux financement des activités politiques et des campagnes électorales.

\section{Procédures d'envoi des rapports au Parlement}

L'envoi des rapports du SAO au Parlement est encadré par des procédures, tandis que les modalités de traitement des rapports d'audit par le Parlement sont fondées sur les Règles de procédure (Règlement intérieur) du Parlement croate.

Les représentants du SAO assistent aux débats des commissions sur les rapports d'audit et, dans le discours d'ouverture, ils exposent les résultats de l'audit.

Les rapports d'audit sont également débattus en séance plénière, à laquelle assistent également les représentants du SAO, et l'Auditeur général prononce le discours d'ouverture. 
Nombre et type de rapports

\begin{tabular}{|c|c|c|c|c|c|c|c|}
\hline & $\begin{array}{l}\text { Rapports } \\
\text { d'audit } \\
\text { financier }\end{array}$ & $\begin{array}{c}\text { Rapports d'audit } \\
\text { de conformité }\end{array} \mid$ & $\begin{array}{l}\text { Rapports d'audit } \\
\text { de performance }\end{array}$ & $\begin{array}{c}\text { Autres rapports } \\
\text { ou documents }\end{array}$ & $\begin{array}{c}\text { Rapports } \\
\text { d'activité } \\
\text { annuels }\end{array}$ & $\begin{array}{c}\text { Rapports } \\
\text { demandés par } \\
\text { le Parlement }\end{array}$ & Remarques \\
\hline $\begin{array}{c}\text { Nombre de } \\
\text { rapports soumis }\end{array}$ & 237 & 0 & 36 & $\begin{array}{c}141 \text { examens } \\
\text { de rapports } \\
\text { financiers } \\
+ \\
28 \text { audits de } \\
\text { suivi }\end{array}$ & 1 & 0 & \\
\hline $\begin{array}{l}\text { Nombre de } \\
\text { rapports discutés } \\
\text { par le Parlement }\end{array}$ & 237 & - & 36 & $(141+28)^{*}$ & 1 & - & $\begin{array}{c}\text { * Dans le cadre } \\
\text { de la discussion } \\
\text { sur le rapport } \\
\text { de travail } \\
\text { annuel }\end{array}$ \\
\hline $\begin{array}{l}\text { Soumis au } \\
\text { Président }\end{array}$ & 0 & - & 0 & 0 & 0 & - & \\
\hline $\begin{array}{l}\text { Soumis au } \\
\text { Président de la } \\
\text { commission } \\
\text { spécialisée }\end{array}$ & 0 & - & 0 & 0 & 0 & - & \\
\hline $\begin{array}{c}\text { Copies envoyées } \\
\text { aux autres } \\
\text { commissions } \\
\text { concernées }\end{array}$ & O* & - & O* & O* & O* & - & $\begin{array}{c}\text { * La distribution } \\
\text { aux } \\
\text { commissions } \\
\text { compétentes } \\
\text { est organisée } \\
\text { par le } \\
\text { Parlement }\end{array}$ \\
\hline $\begin{array}{c}\text { Notes de synthèse } \\
\text { destinées à la } \\
\text { commission } \\
\text { parlementaire }\end{array}$ & $\mathrm{N}$ & - & $\mathrm{N}$ & $\mathrm{N}$ & $\mathrm{N}$ & - & \\
\hline $\begin{array}{c}\text { Résumés soumis au } \\
\text { Parlement }\end{array}$ & 0 & - & O* & O* & O* & - & \begin{tabular}{|c|}
$*$ Le résumé fait \\
toujours partie \\
intégrante du \\
rapport d'audit
\end{tabular} \\
\hline
\end{tabular}

3) Procédures parlementaires utilisées dans le traitement des rapports de l'ISC

Après la discussion en séance plénière, le Parlement croate émet une conclusion, le gouvernement étant alors tenu de réfléchir, dans une période déterminée, à la mise en œuvre des recommandations faites par le SAO. Ce document est publié sur le site Internet du Parlement (avec toutes les autres conclusions). 
Toutes les séances plénières sont entièrement diffusées (" en direct ») sur le site Internet du Parlement croate, ce qui signifie que l'ensemble des débats et des conclusions sont parfaitement transparents pour le public.

\section{Personnel}

Les commissions parlementaires sont généralement assistées par un petit nombre de collaborateurs qui contribuent à l'élaboration des documents et des informations nécessaires aux débats, qui serviront aux opinions des commissions présentées ensuite en séance plénière.

\section{Participation de l'ISC à l'examen de ses rapports par le Parlement}

Avant les débats, le SAO donne des explications sur les rapports d'audit. Par ailleurs, les représentants du SAO assistent régulièrement aux réunions parlementaires pendant lesquelles sont débattus les rapports du SAO.

Les représentants du Bureau national d'audit participent aux discussions en commission et, lors du discours d'ouverture, ils présentent les résultats de l'audit. Sont présents aux réunions des commissions : l'Auditeur général (toujours aux réunions de la Commission des finances et du budget, et aux autres commissions en fonction du thème examiné), L'Auditeur général adjoint et les coordinateurs des audits.

Les rapports d'audit sont également débattus en séance plénière, à laquelle assistent également les représentants du SAO, dont l'Auditeur général chargé du discours d'ouverture.

\section{4) Rapports de I'ISC}

Dans sa communication avec le Parlement, le SAO se concentre sur des problèmes systémiques.

Le système de vérification du contrôle interne fait intégralement partie de chaque audit. En s'appuyant sur la vérification des contrôles financiers internes, les contrôleurs publics déterminent les domaines de risques.

Tous les rapports d'audit contiennent des conclusions (audits de performance) et des opinions claires (audits financiers).

\section{5) Suivi des rapports de I'ISC}

Il existe plusieurs méthodes et niveaux de suivi :

Le représentant juridique de l'entité contrôlée répond officiellement au SAO des actions prises suite aux constatations de l'audit au plus tard 60 jours après la réception du rapport.

La mise en œuvre des recommandations du SAO est vérifiée à chaque audit, en tant que composante normale de la procédure de contrôle. Tout rapport d'audit contient des informations sur les constatations et les recommandations émises lors des précédents audits, en indiquant les recommandations mises en œuvre, celles en cours d'exécution et celles qui n'ont reçu aucune réponse.

Le rapport de travail annuel du SAO indique pour chaque groupe de thèmes contrôlés le nombre de recommandations donné, le nombre mis en œuvre, le nombre en cours d'exécution et le nombre non pris en compte. Il donne également des informations sur les constations et les recommandations liées aux systèmes de contrôle internes. 
Le SAO exécute également des audits de suivi spéciaux (voir le rapport de travail annuel, www.revizija.hr)

Pour terminer, le Parlement croate analyse les recommandations et leur mise en œuvre.

\section{Audits réalisés à la demande du Parlement}

Les dispositions prévues par la Loi sur le SAO permettent à ce dernier de réaliser des audits à la demande du Parlement croate si l'Auditeur général décide que la demande est justifiée. Depuis l'adoption de la Loi sur le SAO, le Parlement croate n'a pas demandé d'audit.

\section{6) Programme de travail}

Le plan de contrôle ne donne pas lieu à des consultations avec le Parlement. L'Article 9 de la Loi sur le SAO stipule que I'Auditeur général remet au Parlement le plan stratégique et le programme de travail annuel du SAO.

\section{7) Niveau et fréquence des échanges}

II y a régulièrement des échanges pendant les séances de la Commission des finances et du budget et celles des autres commissions; ces échanges ont pour but de discuter des rapports soumis par le SAO pendant l'année. Sont présents aux réunions des commissions : I'Auditeur général (toujours aux réunions de la Commission des finances et du budget, et aux autres commissions en fonction du thème examiné), l'Auditeur général adjoint et les coordinateurs des audits.

\section{8) Bonnes pratiques}

Il y a trois ans, à l'initiative du SAO, la Commission des finances et du budget a accepté de modifier la fréquence de présentation des rapports au Parlement et de leur publication, ceci en raison des obligations émanant de la nouvelle Loi sur la liberté de l'information, et pour améliorer l'efficience et la qualité de la communication entre le Parlement croate et le SAO. En conséquence, le SAO soumet les rapports d'audit au Parlement immédiatement après leur achèvement et il les publie pratiquement en même temps sur les sites Internet.

\section{9) Perspective}

Aucun point faible n'est à relever, uniquement des domaines appelant des améliorations potentielles, par exemple :

Mettre en place une commission / sous-commission parlementaire relative à l'audit, spécifiquement dédiée aux rapports d'audit du SAO.

Inviter des responsables des entités contrôlées aux débats des commissions sur les rapports d'audit et inscrire cette procédure dans la pratique courante.

Le SAO a entamé des discussions sur ces deux questions avec le Président du Parlement ainsi qu'avec le responsable et les membres de la Commission des finances et du budget de l'État. Le SAO a organisé un voyage d'étude à l'intention d'un groupe de parlementaires pour visiter le NAO du Royaume-Uni et le Parlement britannique; ces organes ont présenté leurs méthodes de communication et leur fonctionnement comme exemple de bonnes pratiques. 
10) Y-a-t-il d'autres éléments concernant les relations actuelles entre l'ISC et le Parlement que vous souhaiteriez souligner, et quelles a été leur évolution au cours des cinq dernières années?

Voir les sections 9 et 10 . 


\section{CHYPRE $^{1}$}

Bureau d'audit de la République de Chypre

\section{1) Cadre juridique}

Au regard des obligations de I'ISC envers le Parlement, le cadre juridique du SAI est défini par la Constitution de la République de Chypre. L'Auditeur général soumet le rapport annuel au Président de la République, qui le dépose devant le Parlement.

\section{2) Modalités de soumission des rapports de l'ISC au parlement}

L'ISC de Chypre soumet un rapport annuel qui contient la synthèse des constatations et des recommandations de tous ses travaux (audits financiers, audits de conformité, audits de performance). Conformément aux différentes lois, des rapports détaillés individuels portant sur le contrôle des principaux organes institutionnels sont soumis au Parlement.

\section{Procédures d'envoi des rapports au Parlement}

L'envoi des rapports au Parlement n'est régi par aucune procédure particulière autre que celles stipulées dans la Constitution. L'Auditeur général assiste régulièrement aux réunions de la Commission des comptes publics où sont débattues les problématiques soulevées par le rapport annuel ainsi que toute autre question.

\footnotetext{
Note de la Turquie

L'information transmise dans le présent document et qui fait référence à « Chypre » concerne la partie sud de l'île. II n'y a pas d'autorité unique représentant à la fois la population turque et la population grecque chypriote de l'île. La Turquie reconnaît la République turque du nord de Chypre (TRNC). Jusqu'à ce qu'une solution durable et équitable soit trouvée au sein des Nations-Unies, la Turquie restera sur sa position au regard de la " question chypriote».

Note de tous les États membres de l'Union européenne, de l'OCDE et de l'Union européenne

La République de Chypre est reconnues par tous les membres des Nations-Unies à l'exception de la Turquie. L'information transmise dans le présent document concerne la région sous le contrôle effectif du gouvernement de la République de Chypre.
} 
Nombre et type de rapports

\begin{tabular}{|c|c|c|c|c|c|c|c|}
\hline & $\begin{array}{l}\text { Rapports } \\
\text { d'audit } \\
\text { financier }\end{array}$ & $\begin{array}{l}\text { Rapports d'audit } \\
\text { de conformité }\end{array}$ & $\begin{array}{l}\text { Rapports d'audit } \\
\text { de performance }\end{array}$ & $\begin{array}{c}\text { Autres rapports } \\
\text { ou documents }\end{array}$ & $\begin{array}{c}\text { Rapports } \\
\text { d'activité } \\
\text { annuels }\end{array}$ & $\begin{array}{c}\text { Rapports } \\
\text { demandés par } \\
\text { le Parlement }\end{array}$ & Remarques \\
\hline $\begin{array}{c}\text { Nombre de } \\
\text { rapports soumis }\end{array}$ & 10 & $\begin{array}{l}\text { Inclus dans le } \\
\text { rapport annuel }\end{array}$ & 2 & -- & -- & & \\
\hline $\begin{array}{c}\text { Nombre de } \\
\text { rapports discutés } \\
\text { par le Parlement }\end{array}$ & 10 & Voir ci-dessus & 2 & - & - & & \\
\hline $\begin{array}{l}\text { Soumis au } \\
\text { Président }\end{array}$ & $\mathrm{N}$ & $\mathrm{N}$ & $\mathrm{N}$ & $\mathrm{N}$ & $\mathrm{N}$ & & \\
\hline $\begin{array}{l}\text { Soumis au } \\
\text { Président de la } \\
\text { commission } \\
\text { spécialisée }\end{array}$ & $\mathrm{N}$ & $\mathrm{N}$ & $\mathrm{N}$ & $\mathrm{N}$ & $\mathrm{N}$ & & \\
\hline $\begin{array}{c}\text { Copies envoyées } \\
\text { aux autres } \\
\text { commissions } \\
\text { concernées }\end{array}$ & $\mathrm{N}$ & $\mathrm{N}$ & $\mathrm{N}$ & $\mathrm{N}$ & $\mathrm{N}$ & & \\
\hline $\begin{array}{c}\text { Notes de synthèse } \\
\text { destinées à la } \\
\text { commission } \\
\text { parlementaire }\end{array}$ & $\mathrm{N}$ & $\mathrm{N}$ & $\mathrm{N}$ & $\mathrm{N}$ & $\mathrm{N}$ & & \\
\hline $\begin{array}{c}\text { Résumés soumis au } \\
\text { Parlement }\end{array}$ & $\mathrm{N}$ & $\mathrm{N}$ & $\mathrm{N}$ & $\mathrm{N}$ & $\mathrm{N}$ & & \\
\hline
\end{tabular}

3) Procédures parlementaires utilisées dans le traitement des rapports de l'ISC

Lorsque la Commission des comptes publics reçoit le rapport annuel, elle lance une procédure pour débattre du rapport avec les ministères sectoriels et les principaux organismes semi-publics. L'Auditeur général est présent à toutes ces réunions. Les entités contrôlées et les autres parties prenantes sont invitées à assister aux réunions et à s'exprimer devant la commission.

\section{Personnel}

La Commission des comptes publics comprend des parlementaires de tous les partis, qui ont leur assistant personnel. En concertation avec les membres de la commission, le Secrétaire de la Commission des comptes publics prépare les questions pour faciliter les débats.

\section{Participation de l'ISC à l'examen de ses rapports par le Parlement}

L'Auditeur général est présent à toutes les réunions de la Commission des comptes publics et il participe activement au débat, avec la volonté de résoudre les problèmes évoqués dans les rapports. 


\section{4) Rapports de l'ISC}

Le système de contrôle interne des entités contrôlées est examiné, et tout point faible est consigné et inclus dans le rapport annuel.

\section{5) Suivi des rapports de l'ISC}

L'ISC pilote de manière régulière les conclusions et les recommandations qu'elle formule auprès des entités contrôlées. Les entités contrôlées sont juridiquement contraintes de remettre, avec leur budget annuel, une déclaration de conformité avec les recommandations de I'ISC, I'ensemble étant soumis à l'adoption du Parlement.

\section{6) Audits réalisés à la demande du Parlement}

II n'existe pas de disposition juridique permettant au Parlement de demander un audit parce que le SAI est indépendant. Toutefois, le SAl et le Parlement coopèrent étroitement, et toute suggestion du Parlement est prise en compte.

\section{7) Programme de travail}

L'ISC ne consulte pas le Parlement sur son programme de travail annuel en matière de contrôle.

\section{8) Niveau et fréquence des échanges}

L'Auditeur général assiste régulièrement aux réunions de la Commission des comptes publics, à savoir une fois par semaine.

\section{9) Bonnes pratiques}

L'ISC et l'Auditeur général entretiennent des contacts étroits avec le Président de la Commission des comptes publics. Toute demande d'audit / d'enquête par un membre de la Commission est exécutée sans délai. 


\section{RÉPUBLIQUE TCHÈQUE}

Bureau supérieur de contrôle

\section{1) Cadre juridique}

Le bureau supérieur de contrôle tchèque (SAO) est tenu par la Loi relative au Bureau supérieur de contrôle $N^{\circ} 166 / 1993$ d'envoyer au gouvernement son plan de contrôle annuel, son rapport annuel, l'état du compte final de l'État, la déclaration de compte-rendu sur le développement économique et sur l'exécution du budget de l'État, et tous ses rapports d'audit. Les mêmes documents doivent être présentés aux deux chambres du Parlement, la Chambre des députés (chambre basse) et le Sénat (chambre haute).

Dans la pratique, ces rapports sont envoyés au Premier ministre et aux présidents des deux chambres. À la demande de l'une des chambres ou de l'une de ses instances (par exemple une commission), le SAO formule dans le délai convenu des opinions sur la réglementation proposée, au regard notamment de la gestion budgétaire, de la comptabilité, des statistiques de l'État et de la performance du contrôle, et des activités de supervision et d'inspection. Le Président du SAO peut assister aux réunions des deux chambres ou de leurs instances lors des délibérations sur les propositions ou les opinions soumises par le SAO. Le Président du SAO est habilité à prendre la parole aux réunions. Lorsque I'une des chambres ou I'une de ses instances convie le Président du SAO à venir à ses réunions, il doit s'exécuter. Le Président du SAO peut par ailleurs participer aux réunions gouvernementales lorsque ces dernières discutent des rapports d'audit. II a le droit de prendre la parole à ces réunions.

\section{2) Modalités de soumission des rapports de l'ISC au parlement}

Le SAO exécute une quarantaine d'audits par an. II rédige un seul rapport pour chaque audit, appelé par la Loi sur le SAO une "conclusion" d'audit. La conclusion d'audit est un rapport écrit qui résume et évalue les faits relevés au cours du contrôle exécuté. La conclusion d'audit est un document public. Les rapports d'audit sont envoyés au gouvernement et aux deux chambres parlementaires après avoir été adoptés par le Conseil du SAO. Le gouvernement et les deux chambres parlementaires ou leurs instances peuvent également demander des protocoles d'audit. Le protocole d'audit contient un descriptif factuel et répertorie les défauts trouvés, en précisant les réglementations qui n'ont pas été respectées. Le protocole d'audit n'est pas rendu public et il sert de base aux conclusions d'audit (rapport d'audit public). Le gouvernement discute tous les rapports d'audit. Au Parlement, les rapports d'audit sont discutés par la Commission chargée du contrôle budgétaire. Cette commission de la Chambre des députés est constituée par la loi pour chaque législature. La Commission décide du nombre de rapports d'audit à débattre. Elle a traité 25 rapports d'audit en 2015, et 13 en 2014. Les autres commissions ne s'occupent que très rarement des rapports d'audit.

Le rapport d'audit est officiellement envoyé au Président de la chambre, qui le transmet à la Commission de contrôle budgétaire. Le SAO travaille en étroite collaboration avec cette commission.

Le plan de contrôle annuel est envoyé au Parlement après avoir été approuvé par le Conseil du SAO à la fin de l'année calendaire.

Le rapport annuel est envoyé au Parlement avant le 31 mars. Il est débattu par la Commission de contrôle budgétaire et par la chambre haute du Parlement.

L'état du compte final de l'État est envoyé au Parlement quatre mois après que le gouvernement a soumis le compte final à la Chambre des députés. 
La déclaration de compte-rendu sur le développement économique et sur l'exécution du budget de l'État est envoyée au Parlement un mois après que le gouvernement a soumis le rapport à la Chambre des députés.

\section{Procédures régissant l'envoi des rapports au Parlement}

Voir ci-dessus pour connaître les procédures d'envoi des rapports au Parlement. 
Nombre et type de rapports

\begin{tabular}{|c|c|c|c|c|c|c|c|}
\hline & $\begin{array}{l}\text { Rapports } \\
\text { d'audit } \\
\text { financier }\end{array}$ & \begin{tabular}{|l|} 
Rapports d'audit \\
de conformité
\end{tabular} & $\begin{array}{l}\text { Rapports d'audit } \\
\text { de performance }\end{array}$ & $\begin{array}{l}\text { Autres rapports } \\
\text { ou documents }\end{array}$ & $\begin{array}{l}\text { Rapports } \\
\text { d'activité } \\
\text { annuels }\end{array}$ & $\begin{array}{l}\text { Rapports } \\
\text { demandés par } \\
\text { le Parlement }\end{array}$ & Remarques \\
\hline $\begin{array}{c}\text { Nombre de } \\
\text { rapports soumis }\end{array}$ & 4 & 30 & 6 & 2 & 1 & 0 & \\
\hline $\begin{array}{c}\text { Nombre de } \\
\text { rapports discutés } \\
\text { par le Parlement }\end{array}$ & 4 & 16 & 5 & 2 & 1 & 0 & $\begin{array}{c}\text { Les rapports } \\
\text { d'audit sont } \\
\text { discutés pas } \\
\text { seulement la } \\
\text { même année } \\
\text { où le SAO les } \\
\text { envoie au } \\
\text { Parlement }\end{array}$ \\
\hline $\begin{array}{l}\text { Soumis au } \\
\text { Président }\end{array}$ & 0 & 0 & 0 & 0 & 0 & 0 & \\
\hline $\begin{array}{c}\text { Soumis au } \\
\text { Président de la } \\
\text { commission } \\
\text { spécialisée }\end{array}$ & $\mathrm{N}$ & $\mathrm{N}$ & $\mathrm{N}$ & $\mathrm{N}$ & $\mathrm{N}$ & $\mathrm{N}$ & $\begin{array}{c}\text { Le rapport } \\
\text { d'audit est } \\
\text { officiellement } \\
\text { envoyé au } \\
\text { Président de } \\
\text { la chambre, } \\
\text { qui le } \\
\text { transmet à la } \\
\text { Commission } \\
\text { de contrôle } \\
\text { budgétaire }\end{array}$ \\
\hline $\begin{array}{c}\text { Copies envoyées } \\
\text { aux autres } \\
\text { commissions } \\
\text { concernées }\end{array}$ & $\mathrm{N}$ & $\mathrm{N}$ & $\mathrm{N}$ & $\mathrm{N}$ & $\mathrm{N}$ & $\mathrm{N}$ & \\
\hline $\begin{array}{c}\text { Notes de synthèse } \\
\text { destinées à la } \\
\text { commission } \\
\text { parlementaire }\end{array}$ & $\mathrm{N}$ & $\mathrm{N}$ & $\mathrm{N}$ & $\mathrm{N}$ & $\mathrm{N}$ & $\mathrm{N}$ & \\
\hline $\begin{array}{c}\text { Résumés soumis au } \\
\text { Parlement }\end{array}$ & $\mathrm{N}$ & $\mathrm{N}$ & $\mathrm{N}$ & $\mathrm{N}$ & $\mathrm{N}$ & $\mathrm{N}$ & \\
\hline
\end{tabular}

3) Procédures parlementaires utilisées dans le traitement des rapports de l'ISC

Au Parlement, les rapports d'audit sont débattus au sein de la Commission de contrôle budgétaire. C'est la commission de la Chambre des députés qui est constituée par la loi pour chaque législature. Elle a été 
constituée pour la première fois en 2006. La tradition veut qu'elle soit présidée par un membre de l'opposition du parti au pouvoir ou par une coalition.

Elle décide du nombre de rapports d'audit à débattre. Elle a traité 25 rapports d'audit en 2015, et 13 en 2014.

Les autres commissions ne s'occupent que très rarement des rapports d'audit (un ou deux rapports d'audit par législature).

Le Président du SAO, le membre du SAO qui a réalisé l'audit, et également les représentants de l'entité contrôlée sont invités à la réunion de la Commission de contrôle budgétaire lorsque les rapports du SAO sont débattus. Les représentants de l'entité contrôlée sont essentiellement le ministre adjoint, ou parfois un autre ministre.

La Commission de contrôle budgétaire adopte une résolution pour tout rapport d'audit débattu.

Avant la réunion, l'un des membres de la commission est choisi pour être le rapporteur. II consulte principalement le responsable de l'audit du SAO. La commission étant habilitée à faire appel à des experts externes, elle peut leur demander des attestations et les convier aux réunions de la commission. Ces réunions sont publiques. La commission peut également demander n'importe quels documents (même confidentiels) à l'organisme contrôlé.

\section{Personnel}

Le personnel de la Commission de contrôle budgétaire se compose de trois personnes qui ne fournissent qu'une assistance administrative. Le soutien expert est donné par le SAO, par les organismes contrôlés ou par des experts externes indépendants.

\section{Participation de I'ISC à l'examen de ses rapports par le Parlement}

Le Président du SAO, le responsable de l'audit du SAO et les contrôleurs qui ont participé à l'audit assistent à la réunion de la commission où sont débattus les rapports d'audit du SAO.

Le membre de la commission (le rapporteur) peut demander au préalable l'assistance du personnel du SAO.

\section{4) Rapports de I'ISC}

Le SAO exécute une quarantaine d'audits par an. Pour chaque audit, il rédige un seul rapport, appelé par la Loi sur le SAO une "conclusion d'audit ". La conclusion d'audit est un rapport écrit qui résume et évalue les faits relevés au cours du contrôle exécuté. Le rapport d'audit public reprend les constatations. Tous les rapports d'audit contiennent des conclusions.

Outre divers rapports, déclarations et autres matériaux, le SAO établit chaque année un rapport pour I'Union européenne, à savoir le rapport sur la gestion financière de l'Union européenne en République tchèque.

\section{5) Suivi des rapports de l'ISC}

Le SAO évalue les mesures correctives proposées et adoptées par les entités contrôlées. 
Le gouvernement étudie tous les rapports d'audit et adopte une résolution pour chacun. La majorité des résolutions demande au ministère concerné d'adopter des mesures correctives (pour $88 \%$ des cas en 2015 et pour $86 \%$ des cas en 2014). Très souvent, ce ministère envoie au gouvernement un rapport sur l'application de ces mesures à la fin de la période dévolue aux mesures correctives.

Le SAO évalue ensuite ces mesures. Cette évaluation concerne les audits individuels. Dans les audits de suivi, le SAO vérifie la mise en œuvre des mesures adoptées. Cette vérification fait toujours partie du rapport d'audit (portant sur l'audit de suivi).

Lors de la seconde étape, la Commission de contrôle budgétaire évalue les mesures correctives. La Commission examine les rapports d'audit quelques mois après sa réunion avec le gouvernement de manière à évaluer l'avancement des travaux.

\section{6) Audits réalisés à la demande du Parlement}

Le gouvernement et les deux chambres parlementaires ou leurs instances peuvent demander au SAO de réaliser un contrôle. Ce processus est désigné sous le terme de "suggestion qualifiée ". Le Conseil du SAO peut prendre la décision d'intégrer la suggestion dans le plan de contrôle annuel, d'utiliser la suggestion dans l'audit en cours ou dans la préparation du prochain plan de contrôle annuel, ou de ne pas exécuter l'audit.

Entre 2006 et 2015, le SAO a reçu 26 suggestions : 2 du gouvernement, 4 du Sénat et 20 de la Chambre des députés. La Commission de contrôle budgétaire en a suggéré 13 . Sur ces 26 suggestions, 18 ont été intégrées dans le plan de contrôle annuel ou seront utilisées dans la préparation d'un prochain plan de contrôle annuel. Dans la même période, le SAO a mené 362 audits.

\section{7) Programme de travail}

Voir également la réponse précédente. La majorité des audits sont planifiés au sein des activités analytiques du SAO. Les suggestions qualifiées ne représentent que $5 \%$ des audits inscrits au plan de contrôle annuel.

\section{8) Niveau et fréquence des échanges}

En 2015, la Commission de contrôle budgétaire a tenu 8 réunions officielles. Outre les réunions officielles, le membre de la commission (le rapporteur) rencontre le responsable de l'audit du SAO. II y a également de nombreuses réunions officieuses entre les représentants du SAO et les membres de la commission et d'autres représentants parlementaires.

Le SAO invite également les représentants de la Commission aux manifestations qu'il organise, par exemple des séminaires et des conférences.

\section{9) Bonnes pratiques}

Pendant les réunions officielles du gouvernement, les rapports d'audit du SAO font l'objet de débats de fond. Le SAO peut contribuer à renforcer ses recommandations auprès de l'exécutif. En collaboration avec la Commission parlementaire, le SAO est en mesure de vérifier si les recommandations et les mesures qui s'y rapportent ont été adoptées et mises en œuvre. 


\section{0) Perspective}

Dans certains cas, le Parlement discute les rapports d'audit malheureusement trop longtemps après leur publication. Les représentants du SAO s'efforcent actuellement d'améliorer cette situation.

11) Y-a-t-il d'autres éléments concernant les relations actuelles entre l'ISC et le Parlement que vous souhaiteriez souligner, et quelles a été leur évolution au cours des cinq dernières années?

II serait utile que les rapports d'audit soient discutés non seulement pendant les réunions de la Commission de contrôle budgétaire, mais également lorsque se réunissent les autres commissions compétentes. 


\section{DANEMARK}

Bureau national d'audit

\section{1) Cadre juridique}

Le cadre juridique est essentiellement défini à la Section 8.1. de la Loi relative à l'Auditeur général(http://uk.rigsrevisionen.dk/legislation/the-auditor-general-act/):

L'Auditeur général a pour tâche d'assister la Commission des comptes publics lorsque celle-ci examine les comptes de l'État. L'Auditeur général étudie et dresse un rapport sur les questions que la Commission des comptes publics souhaite clarifier. Cette Commission et l'Auditeur général peuvent demander des réunions conjointes. À la demande de la Commission des comptes publics, l'Auditeur général participe aux consultations avec les commissions du Parlement (Folketing) et la Commission des comptes publics.

\section{2) Modalités de soumission des rapports de l'ISC au parlement}

La méthode de soumission des rapports est définie à la Section 17 de la Loi relative à l'Auditeur général :

(1) L'Auditeur général examine l'exhaustivité des comptes publics, puis il compare les chiffres des crédits avec les écritures comptables. L'Auditeur général présente un rapport à la Commission des comptes publics dans le délai défini par la Commission des comptes publics et par l'Auditeur général.

(2) L'Auditeur général présente à la Commission des comptes publics un rapport d'exécution des contrôles qu'il souhaite mettre en évidence au vue de leur importance financière ou générale.

(3) Un cas inclus dans le rapport de L'Auditeur général sera présenté au ministre concerné au plus tard quatre semaines avant la soumission du rapport. Si le cas concerne les sociétés à responsabilité limitée mentionnées à la section 4(1) (i), il sera également présenté au conseil du comté. Si le cas concerne les sociétés à responsabilité limitée mentionnées à la section $4(2)$, première phrase, il sera également présenté au conseil d'administration de la société. Aucune présentation n'est exigée si le cas a déjà fait l'objet d'une correspondance écrite.

(4) L'Auditeur général dépose devant la Commission des comptes publics un rapport annuel sur ses activités, au moment convenu avec la Commission.

\section{Procédures d'envoi des rapports au Parlement}

L'ISC soumet tous les rapports à la Commission des comptes publics (PAC). Le contrôleur chargé du rapport le présente aux membres de la PAC. La PAC se réunit une fois par mois. 
Nombre et type de rapports

\begin{tabular}{|c|c|c|c|c|c|c|c|}
\hline & $\begin{array}{l}\text { Rapports } \\
\text { d'audit } \\
\text { financier }\end{array}$ & $\begin{array}{c}\text { Rapports d'audit } \\
\text { de conformité }\end{array}$ & $\begin{array}{l}\text { Rapports d'audit } \\
\text { de performance }\end{array}$ & \begin{tabular}{|c|} 
Autres rapports \\
ou documents
\end{tabular} & $\begin{array}{l}\text { Rapports } \\
\text { d'activité } \\
\text { annuels }\end{array}$ & $\begin{array}{l}\text { Rapports } \\
\text { demandés } \\
\text { par le } \\
\text { Parlement }\end{array}$ & Remarques \\
\hline $\begin{array}{c}\text { Nombre de } \\
\text { rapports soumis }\end{array}$ & 1 & 15 & 11 & - & 1 & 5 & À la PAC \\
\hline $\begin{array}{c}\text { Nombre de } \\
\text { rapports discutés } \\
\text { par le Parlement }\end{array}$ & 1 & 15 & 11 & - & 1 & 5 & À la PAC \\
\hline $\begin{array}{l}\text { Soumis au } \\
\text { Président }\end{array}$ & 0 & 0 & 0 & 0 & 0 & 0 & \\
\hline $\begin{array}{l}\text { Soumis au } \\
\text { Président de la } \\
\text { commission } \\
\text { spécialisée }\end{array}$ & $\mathrm{N}$ & $\mathrm{N}$ & $\mathrm{N}$ & $\mathrm{N}$ & $\mathrm{N}$ & $\mathrm{N}$ & C'est possible \\
\hline $\begin{array}{c}\text { Copies envoyées } \\
\text { aux autres } \\
\text { commissions } \\
\text { concernées }\end{array}$ & $\mathrm{N}$ & $\mathrm{N}$ & $\mathrm{N}$ & $\mathrm{N}$ & $\mathrm{N}$ & $\mathrm{N}$ & C'est possible \\
\hline $\begin{array}{c}\text { Notes de synthèse } \\
\text { destinées à la } \\
\text { commission } \\
\text { parlementaire }\end{array}$ & $\mathrm{N}$ & $\mathrm{N}$ & $\bar{N}$ & $\bar{N}$ & $\mathrm{~N}$ & $\mathrm{~N}$ & C'est possible \\
\hline $\begin{array}{c}\text { Résumés soumis au } \\
\text { Parlement }\end{array}$ & $\mathrm{N}$ & $\mathrm{N}$ & $\mathrm{N}$ & $\mathrm{N}$ & $\mathrm{N}$ & $\mathrm{N}$ & \\
\hline
\end{tabular}

3) Procédures parlementaires utilisées dans le traitement des rapports de l'ISC

Des procédures établies sont dévolues au traitement des rapports de I'ISC par la PAC. Les règles sont définies dans la Loi sur la PAC, la Loi sur l'Auditeur général et dans les règles de procédure du Parlement.

La PAC a pour mission de s'occuper des rapports de l'ISC. Elle est présidée par le membre qui a le plus d'ancienneté dans la Commission.

La Commission ne nomme pas de rapporteur.

II n'y a pas d'auditions auxquelles serait conviée l'entité contrôlée ou d'autres personnes appropriées afin d'apporter des clarifications ou un complément d'informations.

La PAC peut demander un complément d'informations ou des clarifications.

Le débat sur les rapports de l'ISC se déroule en commission. 
Selon le cas, des commissions autres que la PAC peuvent également analyser les rapports de I'ISC.

Le rapport de I'ISC est publié par la PAC, accompagné des commentaires de la Commission.

II existe une procédure de décharge établie. La PAC rédige un rapport spécial en s'appuyant sur les mémorandums de I'ISC.

\section{Personnel}

La PAC (six hommes/femmes politiques) est assistée de trois assistants parlementaires (deux cadres spécialisés et un employé de bureau). Ils sont chargés de toutes les tâches énumérées ci-dessus. http://uk.rigsrevisionen.dk/the-public-accounts-committee/the-public-accounts-committee/

\section{Examen des rapports par le Parlement}

L'ISC assiste aux réunions de la PAC (I'Auditeur général, le Secrétaire général et les auditeurs responsables des rapports). Elle offre des informations et des clarifications sur les questions évoquées dans les rapports.

\section{4) Rapports de I'ISC}

L'ISC établit trois rapports par an.

\section{5) Suivi des rapports de l'ISC}

Les pratiques de suivi sont définies à la Section 18 de la Loi relative à l'Auditeur général :

1) La Commission des comptes publics transmet les rapports indiqués aux sections 17(1) et (2) au Folketing et au ministre concerné pour commentaires. Le rapport mentionné à la section 17(4) doit être soumis au Folketing.

(2) Le ministre dépose devant de la Commission des comptes publics sa réponse quant aux mesures et considérations formulées dans le rapport, dans les délais déterminés par la Commission sur recommandation de l'Auditeur général. Le délai minimum est de deux mois et le délai maximum de quatre mois. Le rapport mentionné à la section 17(1) est assorti d'un délai de deux mois.

(3) Le ministre obtient une déclaration du conseil du comté pour les comtés énoncés à la section 4(1) (i). $\mathrm{Au}$ regard des sociétés à responsabilité limitée mentionnées à la section 4(2), première phrase, le ministre obtient une déclaration du conseil d'administration de la société. Outre cette déclaration, les commentaires du ministre sur la déclaration du conseil du comté ou sur celle du conseil d'administration seront inclus dans la réponse évoquée à la sous-section (2) de la Commission des comptes publics.

(4) La réponse et la déclaration du conseil du comté ou du conseil d'administration de la société à responsabilité limitée mentionnée à la sous-section (3) sont simultanément transmises à l'Auditeur général qui soumet ses commentaires à la Commission des comptes publics dans le mois suivant la réception de ces documents.

(5) Les réponses des ministres et les commentaires de l'Auditeur général constituent une partie du rapport final que la Commission des comptes publics remet au Folketing. Si la Commission le juge approprié, les déclarations mentionnées à la sous-section (3) peuvent également faire partie du rapport final. 


\section{6) Audits réalisés à la demande du Parlement}

Seule la PAC peut demander à l'ISC un audit particulier. Autour de $20 \%$ des rapports de I'ISC sont établis suite à une requête de la PAC.

\section{7) Programme de travail}

L'ISC informe la PAC de son programme de travail annuel relatif aux contrôles. Le plan ne fait l'objet d'aucune consultation, mais la PAC peut faire des suggestions.

\section{8) Niveau et fréquence des échanges}

Il y a régulièrement des échanges (quotidiens) entre le Secrétaire général de I'ISC et le Chef du Secrétariat de la PAC.

L'Auditeur général rencontre la PAC tous les mois.

\section{9) Bonnes pratiques}

Notre procédure de suivi applique les bonnes pratiques.

L'ISC consigne dans un rapport les résultats de l'audit annuel et des principales études à l'intention de la PAC. L'ISC et la PAC suivent ensuite les résultats pour s'assurer que les ministères agissent. Vue à l'échelle internationale, cette procédure de suivi est unique et elle contribue à l'efficacité des audits.

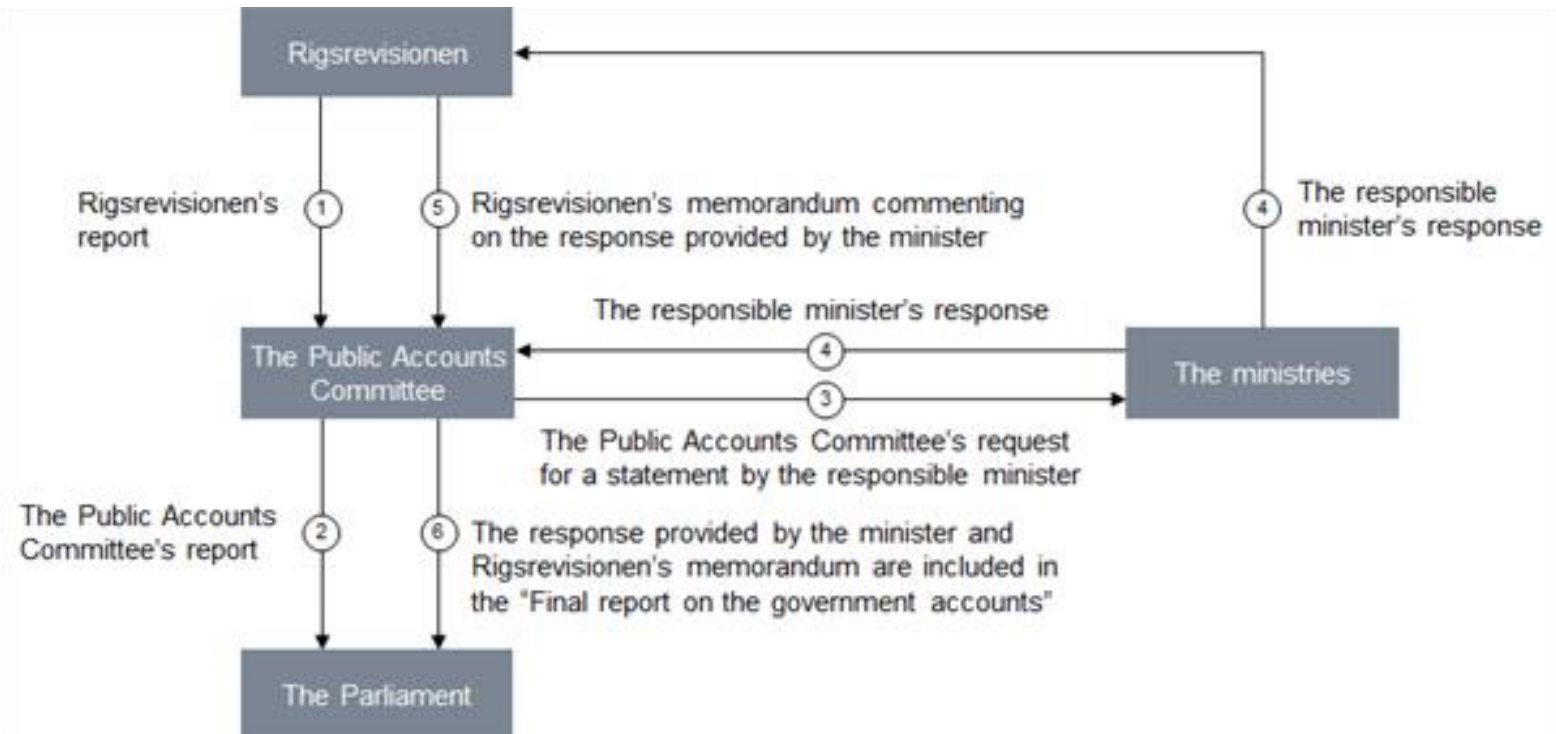

Une fois que I'ISC (Rigsrevisionen) a soumis son rapport à la PAC, les membres de la Commission émettent des commentaires sur le rapport, qui est ensuite transmis au Parlement.

La PAC demande en même temps au ministre compétent de soumettre ses commentaires sur les constatations d'audit. 
Dans les deux à quatre mois qui suivent, selon la décision de la PAC, le ministre doit communiquer au Rigsrevisionen et à la Commission les mesures que prendra le ministère pour se conformer aux recommandations.

En s'appuyant sur cette déclaration, le Rigsrevisionen soumet un mémorandum de suivi à la PAC, lui recommandant soit de clôturer l'affaire, puisque toutes les problématiques ont été traitées de manière satisfaisante, soit qu'il reste des questions en attente auxquelles le Rigsrevisionen a l'intention de donner suite.

Les déclarations ministérielles et les mémorandums de suivi élaborés par le Rigsrevisionen sont rendus publics dans le rapport final sur les comptes de l'État, qui est soumis une fois par an au Parlement par le biais de la PAC. Le rapport sur les comptes de l'État est le document sur lequel s'appuie le Parlement pour approuver les comptes de l'État de l'exercice fiscal.

\section{0) Perspective}

Certains rapports de l'ISC ont été envoyés par la PAC aux autres commissions. Parmi ces commissions, certaines ont demandé une présentation d'un rapport de I'ISC. II semblerait que l'intérêt porté par d'autres commissions aux rapports de I'ISC se renforcera à l'avenir. 


\section{ESTONIE}

Bureau national d'audit

\section{1) Cadre juridique}

L'indépendance du Bureau national d'audit d'Estonie (NAOE) est assurée par la Constitution et la Loi relative au Bureau national d'audit.

Aux termes de la Loi relative au Bureau national d'audit, le NAOE élabore de façon indépendante son programme de contrôle, y compris le calendrier et la nature des vérifications. Personne ne peut contraindre le Bureau national d'audit à exécuter des missions de contrôle forcées. Les parlementaires peuvent poser des questions à l'Auditeur général (AG) sur des thèmes qui les intéressent, ou qui sont dans l'agenda du Parlement et s'inscrivent dans la sphère d'activité du Bureau national d'audit.

La Loi stipule que les travaux du NAOE se basent sur le calendrier de travail approuvé par l'AG. Ce calendrier est disponible sur le site Internet du NAOE, et l'AG le présente à la commission parlementaire restreinte chargée du contrôle du budget de l'État.

Les activités du NAOE sont vérifiées tous les ans par un auditeur nommé par le Parlement. Le NAOE soumet son rapport annuel vérifié au Parlement.

La Constitution stipule également que l'AG doit présenter au Parlement une vue globale de l'utilisation et de la préservation des actifs publics. Chaque automne, l'AG complète ce rapport analytique qui résume une année de travail en faisant un discours devant le Parlement et en répondant aux questions des parlementaires.

\section{2) Modalités de soumission des rapports de l'ISC au parlement}

Au titre de la Loi relative au Bureau national d'audit, la Commission parlementaire restreinte chargée du contrôle du budget de l'État sert de lien entre le NAOE et le Parlement.

L'AG soumet tous les résultats des audits (rapports financiers, de conformité et de performance) à la Commission de contrôle du budget de l'État (adressés au Président de la Commission permanente). Le rapport annuel du NAOE et la synthèse générale de l'utilisation et de la préservation des actifs publics sont soumis au Président du Parlement. Les rapports sont envoyés et discutés individuellement. Un représentant du NAOE et un autre de l'entité contrôlée sont invités à participer à la discussion portant sur les résultats d'audit au sein de la commission restreinte. Les délais d'envoi des rapports à la Commission ne sont pas réglementés.

Les rapports d'audit de performance sont souvent envoyés à d'autres commissions parlementaires, où ils sont débattus en suivant la même procédure. Dans certains cas, les commissions ont tenu des réunions mixtes portant sur les rapports d'audit du NAOE.

\section{Procédures d'envoi des rapports au Parlement}

Les rapports sont envoyés à la Commission de contrôle du budget de l'État sous forme électronique, avec des signatures numériques. 
Le NAOE fait, à huit clos, une présentation d'information sur le rapport d'audit devant les membres de la Commission de contrôle du budget de l'État, normalement une fois par semaine.

Après sa présentation, le rapport d'audit est discuté en audition publique organisée par la Commission restreinte. Ces auditions sont disponibles sur Internet. Les représentants du NAOE résument les résultats d'audit et répondent aux questions posées par les membres de la Commission restreinte. Les représentants de l'entité contrôlée présentent ensuite leur opinion et explications. À la fin de la réunion, le Président de la Commission restreinte résume la discussion et l'information est consignée par écrit. Dans certains cas, il est décidé de tenir une autre réunion sur le même sujet au bout de six mois, ou d'envoyer un courrier de notification à l'institution compétente. 
Nombre et type de rapports

\begin{tabular}{|c|c|c|c|c|c|c|c|}
\hline & $\begin{array}{l}\text { Rapports } \\
\text { d'audit } \\
\text { financier }\end{array}$ & \begin{tabular}{|l|} 
Rapports d'audit \\
de conformité
\end{tabular} & $\begin{array}{l}\text { Rapports d'audit } \\
\text { de performance }\end{array}$ & \begin{tabular}{|l|} 
Autres rapports \\
ou documents
\end{tabular} & $\begin{array}{l}\text { Rapports } \\
\text { d'activité } \\
\text { annuels }\end{array}$ & $\begin{array}{c}\text { Rapports } \\
\text { demandés par } \\
\text { le Parlement }\end{array}$ & Remarques \\
\hline $\begin{array}{c}\text { Nombre de } \\
\text { rapports soumis }\end{array}$ & 18 & 6 & 6 & 2 & 2 & - & \\
\hline $\begin{array}{l}\text { Nombre de } \\
\text { rapports discutés } \\
\text { par le Parlement }\end{array}$ & 2 & \begin{tabular}{|c|}
5 (quelquefois \\
plus d'une fois)
\end{tabular} & $\begin{array}{l}7 \text { (quelquefois } \\
\text { plus d'une fois) }\end{array}$ & & & & \\
\hline $\begin{array}{l}\text { Soumis au } \\
\text { Président }\end{array}$ & - & - & - & & 2 & - & $\begin{array}{c}\text { Il y a eu } \\
\text { également } 2 \\
\text { demandes } \\
\text { d'informations } \\
\text { par les } \\
\text { parlementaires } \\
\text { auxquelles } \\
\text { I'AG a répondu }\end{array}$ \\
\hline $\begin{array}{l}\text { Soumis au } \\
\text { Président de la } \\
\text { commission } \\
\text { spécialisée }\end{array}$ & 18 & 6 & 7 & 2 & 2 & - & \\
\hline $\begin{array}{c}\text { Copies envoyées } \\
\text { aux autres } \\
\text { commissions } \\
\text { concernées }\end{array}$ & 1 & 3 & 6 & & 1 & - & \\
\hline $\begin{array}{c}\text { Notes de synthèse } \\
\text { destinées à la } \\
\text { commission } \\
\text { parlementaire }\end{array}$ & 2 & 5 & 13 & 2 & 2 & - & \\
\hline $\begin{array}{c}\text { Résumés soumis au } \\
\text { Parlement }\end{array}$ & 18 & 6 & 6 & 2 & 2 & - & \\
\hline
\end{tabular}

\section{3) Procédures parlementaires utilisées dans le traitement des rapports de l'ISC}

En vertu de la Loi relative au Bureau national d'audit, le principal point de contact du NAOE au Parlement est la Commission de contrôle du budget de l'État, qui s'assure que les finances et les actifs publics sont utilisés de manière économique, efficiente, efficace et légale.

Les Règles de procédure et le Loi relative au Règlement du Parlement offrent un cadre général à l'organisation des travaux des commissions parlementaires, sans faire spécifiquement référence à la Commission de contrôle du budget de l'État. 
Chaque Commission de contrôle du budget de l'État est le fruit de négociations politiques, qui s'appuient également sur la tradition. Toutes les tendances politiques sont représentées par un ou plusieurs membres. Depuis 2015, il y a eu 6 partis politiques, chacun représenté par un membre. La commission précédente comportait 8 membres issus de 4 partis.

Traditionnellement, le Président de la Commission vient du principal parti d'opposition, tandis que le président adjoint appartient à la coalition.

La Commission restreinte n'affecte pas de rapporteurs aux rapports d'audit, qui sont présentés par des membres du NAOE.

La Commission restreinte organise des auditions publiques auxquelles est conviée l'entité contrôlée ou d'autres personnes appropriées afin d'apporter des clarifications ou un complément d'informations. Le débat sur les rapports du NAOE se déroule au sein de la Commission restreinte, parfois également au sein de commissions spéciales; il est publié sur Internet.

À la fin de la réunion, le Président de la Commission restreinte résume la discussion et l'information est consignée. Suite aux délibérations, la Commission ne prépare généralement pas de document spécial. Elle diffuse parfois un communiqué de presse. Dans certains cas, il a été décidé de tenir une autre réunion sur le même sujet au bout de six mois, ou d'envoyer un courrier de notification à l'institution compétente. Il y a eu deux exceptions depuis la constitution de la Commission en 2004 ; dans ces deux cas, cette dernière a établi son propre rapport sur le rapport du NAOE.

Une fois par an, l'AG présente au Parlement (en séance plénière) une vue globale de l'utilisation et de la préservation des actifs publics. C'est le seul rapport du NAOE débattu en séance plénière.

Le NAOE doit contrôler les comptes annuels présentés dans le rapport consolidé annuel soumis par l'État, ainsi que la régularité des opérations d'ici le 31 août; il soumet ensuite le rapport à la Commission restreinte chargée du contrôle du budget de l'État. Tous les rapports du NAOE sont soumis en premier lieu à la Commission restreinte. Les rapports sont également envoyés au ministère des Finances.

Le ministère des Finances doit soumettre le rapport consolidé annuel de l'État, accompagné d'un projet de résolution sur la distribution de l'excédent de trésorerie non consolidé de l'État. II doit par ailleurs remettre au gouvernement de la République le rapport d'audit publié par le NAOE sur le contrôle des comptes annuels du rapport annuel consolidé de l'État, ainsi que la vérification de la régularité des transactions. Ces documents doivent être approuvés dans les 7 jours ouvrés à compter de la réception du rapport du NAOE. Les rapports sont publiés sur le site Internet immédiatement après leur soumission au Gouvernement de la République. Ce dernier soumet alors le rapport consolidé annuel entériné à l'approbation du Parlement. Le rapport d'audit du NAOE doit être ajouté au rapport consolidé annuel de l'État. Avant de soumettre les rapports au Parlement réuni en séance plénière, ils sont débattus au sein de la Commission de contrôle du budget de l'État et de la Commission des finances.

Lorsque le rapport sur l'exécution du budget de l'État est débattu au Parlement, l'AG fait un discours devant le Parlement réuni en séance plénière et présente une vue globale de l'utilisation et de la préservation des actifs publics. Avant d'adopter le rapport sur l'exécution du budget de l'État, les parlementaires peuvent poser des questions sur les deux rapports annuels du NAOE. 


\section{Personnel}

Les travaux de la Commission de contrôle du budget de l'État sont coordonnés par deux responsables de l'organisation technique des réunions. Ils invitent des représentants des institutions contrôlées et rassemblent des informations supplémentaires, le cas échéant.

\section{Participation de I'ISC à l'examen de ses rapports par le Parlement}

Le NAOE participe à la préparation des auditions de ses rapports en suggérant quelles personnes il conviendrait d'inviter et quels documents supplémentaires les membres de la Commission restreinte pourraient consulter avant l'audition.

Les membres de la Commission restreinte sont informés des résultats de l'audit par des représentants du NAOE. Ces séances d'information se tiennent à huit clos.

La délégation du NAOE se compose du Directeur du contrôle qui a signé le rapport, du Responsable du contrôle et des auditeurs qui ont participé à la vérification.

\section{4) Rapports de I'ISC}

Le NAOE se concentre sur les problèmes génériques / systémiques relatifs aux contrôles et sur la formulation de recommandations visant à améliorer à la fois ses audits et sa communication avec le Parlement.

Le NAOE insiste en permanence sur la pertinence des systèmes de contrôle internes ; il émet une opinion sur les systèmes de contrôle internes des ministères dans son examen annuel des comptes consolidés de l'État.

Les audits offrent aux parties prenantes compétentes des opinions et des recommandations claires.

\section{5) Suivi des rapports de l'ISC}

Le suivi de la mise en œuvre des recommandations est systématiquement suivi par le NAOE. Les divisions d'audit du NAOE conservent une trace des recommandations et récapitulent les actions qui ont été prises pendant l'année. Outre ce récapitulatif, un résumé des résultats des audits depuis les dix dernières années (depuis leur mise en place) vient d'être établi par la division d'audit chargée des autorités locales. Le NAOE prévoit d'étendre ces analyses à d'autres services. Le NAOE n'établit pas de rapport spécifiquement consacré à la mise en œuvre des recommandations. La présentation du pourcentage de mise en œuvre des recommandations a été rendue obligatoire dans le processus budgétaire. Toutefois, il a été difficile d'évaluer l'utilisation directe ou régulière de cette information ainsi que son impact.

La Commission restreinte ne suit pas activement la mise en œuvre des recommandations du NAOE. Dans certains cas, les réunions de suivi ont lieu six mois après l'audition. En 2015, quatre audits ont été débattus lors de réunions supplémentaires. L'un de ces audits a été débattu pendant quatre réunions supplémentaires où étaient également invités des experts et des membres d'autres commissions parlementaires. 


\section{6) Audits réalisés à la demande du Parlement}

La Loi relative au Bureau national d'audit ou tout autre instrument législatif n'habilite pas le Parlement à solliciter des audits auprès du NAOE. Le Parlement peut suggérer des thèmes, mais le NAOE décide en toute indépendance de donner suite ou pas.

Les parlementaires peuvent soumettre des questions écrites aux agents dirigeants de l'État, dont l'AG, sur des sujets qui les intéressent ou qui sont à l'ordre du jour du Parlement et s'inscrivent dans le domaine de responsabilité de l'agent. II est obligatoire de répondre oralement aux interpellations dans une période de 20 jours parlementaires ouvrés ( 3 semaines par mois et 4 jours par semaine), devant le Parlement réuni en séance plénière. Les questions écrites doivent être traitées dans une période de 10 jours parlementaires ouvrés.

\section{7) Programme de travail}

La Commission de contrôle du budget de l'État réunit tous les ans des suggestions d'audit à l'intention du NAOE. II y a d'ordinaire entre 20 et 25 propositions présentées au NAOE. Le NAOE analyse ces propositions et répond à la Commission restreinte en précisant les propositions qui seront intégrées à son programme de travail, les cas qui ont déjà été contrôlés et les propositions auxquelles il ne donnera pas suite, en motivant ses décisions.

Aux termes de la Loi relative au Bureau national d'audit, la demande d'audits auprès du NAOE ne fait pas partie du mandat du Parlement.

\section{8) Niveau et fréquence des échanges}

L'AG s'entretient avec le Président de la Commission de contrôle du budget de l'État généralement 4 à 5 fois par an. Il assiste en personne à la réunion où il présente le nouveau programme de travail à la Commission. Avant de faire son discours devant le Parlement réuni en séance plénière, il présente une vue globale de l'utilisation et de la préservation des actifs publics.

L'AG participe de temps à autre aux auditions tenues par la Commission s'il souhaite souligner l'importance de certains rapports d'audit.

L'AG rencontre le Président du Parlement au moins une fois par an, lorsqu'il lui remet la vue globale annuelle de l'utilisation et de la préservation des actifs publics.

Les échanges quotidiens avec la Commission permanente sont traités par le Responsable de la communication du NAOE.

\section{0) Perspective}

Le principal problème qui se pose au NAOE est que les discussions portant sur les résultats des audits n'ont pas l'influence souhaitée, et souvent ces discussions s'arrêtent à l'audition. Les membres de la Commission ne prennent pas l'initiative de demander des améliorations au gouvernement. Le principe de travail de la Commission est de parvenir à un consensus et, en conséquence, travailler sur des résultats d'audit n'est pas une décision qu'elle prend souvent.

11) Y-a-t-il d'autres éléments concernant les relations actuelles entre I'ISC et le Parlement que vous souhaiteriez souligner, et quelles a été leur évolution au cours des cinq dernières années? 
Le cas du NAOE démontre que travailler avec une commission restreinte ne constitue peut-être pas la méthode la plus efficiente au regard du suivi de la mise en œuvre des recommandations. Dans certains cas, il peut être plus rapide d'obtenir des résultats si les problèmes sont discutés avec des représentants du pouvoir exécutif. 


\section{LA COUR DES COMPTES EUROPÉENNE}

\section{1) Cadre juridique}

En tant qu'institution de I'Union européenne, établie par traité, la Cour des comptes européenne (ECA) réalise ses audits dans un cadre interinstitutionnel défini essentiellement par le Traité sur le fonctionnement de l'Union européenne (TFUE) et le Règlement financier relatif au budget général de I'Union européenne.

Le Traité stipule que l'ECA doit assister le Parlement européen (PEPE) et le Conseil de l'Union européenne (Conseil) dans l'exercice de leur fonction de contrôle de l'exécution du budget et qu'elle peut, à tout moment, soumettre des observations sur des questions spécifiques et émettre des avis à la demande de l'une des institutions de l'UE.

Une composante essentielle de l'assistance que nous fournissons au PE et au Conseil concerne la procédure de décharge donnée à la Commission et à d'autres institutions sur l'exécution du budget de I'Union (Article 319 TFUE). Ce processus se fonde principalement sur notre rapport annuel, et en particulier sur les résultats de la déclaration d'assurance (article 287 TFUE).

Lorsque nous élaborons notre programme de travail, nous tenons compte des besoins des parties prenantes afin de veiller à ce que nos contrôles soient aussi pertinents et utiles que possible. LePE et le Conseil sont deux parties prenantes essentielles, et lorsque nous sélectionnons nos tâches d'audit, nous prenons en considération leurs demandes ou leurs suggestions en même temps que nos autres priorités.

\section{2) Modalités de soumission des rapports de l'ISC au parlement}

Les résultats de nos travaux d'audit sont publiés sur notre site Internet et mis à la disposition du PE et des autres parties prenantes. Nous avons trois principaux produits :

- Des rapports annuels qui présentent essentiellement les résultats de nos audits financiers et de conformité effectués sur le budget de I'Union et les Fonds européens de développement. Nous publions en outre séparément des rapports annuels spécifiques concernant les agences de I'UE, les organismes décentralisés et les entreprises communes ${ }^{32}$.

- Des rapports spéciaux publiés tout au long de l'année, qui présentent les résultats de nos audits sélectionnés portant sur des domaines budgétaires ou sur des thèmes de gestion spécifiques. Il s'agit surtout d'audits de performance ;

- Des avis sur des propositions ou des mises à jour législatives ayant une incidence sur la gestion financière, ainsi que d'autres produits fondés sur des contrôles, telles que les analyses panoramiques, soit à la demande d'une autre institution, soit de notre propre initiative.

Nous publions et remettons également au PE :

- Notre rapport annuel d'activité qui donne une vue d'ensemble de nos principaux résultats et réalisations de l'année ; et

- Notre programme de travail annuel qui définit nos priorités et nos travaux de l'année. 
Nos rapports annuels (incluant les réponses des institutions) sont publiés au Journal officiel de I'Union européenne. Nous en remettons un exemplaire au Président du PE, au Président de la Commission du contrôle budgétaire, et au "rapporteur» désigné pour la décharge. Par ailleurs, nous envoyons individuellement un exemplaire de nos rapports annuels à chacun des membres du Parlement (MEP).

Nous envoyons nos rapports spéciaux au Président du PE, à tous les parlementaires de la Commission du contrôle budgétaire (CONT), ainsi qu'aux autres commissions parlementaires spécialisées, compétentes pour le thème examiné. Nous sélectionnons les sujets de nos audits lors de notre procédure de planification annuelle. Ce faisant, nous tenons compte des points de vue des parties prenantes $-y$ compris ceux du PE - afin de veiller à ce que les rapports qui en découlent soient aussi pertinents et utiles que possible.

En matière de calendrier, le Règlement financier dispose que l'ECA doit transmettre aux autorités responsables (le PE et le Conseil) pour octroyer la décharge, au plus tard le 15 novembre (année $n+1)$, son rapport annuel accompagné des réponses des institutions. Sur recommandation du Conseil, lePE doit, avant le 15 mai de l'année $n+2$, donner décharge à la Commission sur l'exécution du budget de l'année $n$, qui est l'exercice budgétaire examiné.

Le Règlement financier ne prévoit pas de délais particuliers pour la publication des rapports spéciaux (mise à part l'indication qu'ils doivent être terminés dans une période de 13 mois $^{33}$ ). Ainsi, nous les publions tout au long de l'année, en visant à assurer un flux régulier, et non concentré sur certaines périodes.

Au titre de la politique de dématérialisation que nous avons introduite en 2016 , nous n'envoyons plus nos rapports aux parties prenantes sous forme papier, mais à des commissions parlementaires ciblées, aux secrétariats et aux autres parties prenantes, sous la forme de message électronique, le jour même de la publication.

Tous les rapports de l'ECA sont discutés en CONT. De plus, les membres de l'ECA sont invités à présenter leurs rapports spéciaux, et à participer au débat au sein des commissions parlementaires spécialisées qui s'intéressent au sujet.

En 2015, nous avons publié 25 rapports spéciaux sur des thématiques et sujets divers présentant un intérêt particulier. Durant 2015, nous avons transmis au Parlement européen 26 rapports spéciaux (rapports spéciaux de 2014 et 2015), nos rapports annuels sur l'exécution du budget de l'Union pour l'exercice budgétaire et les Fonds de développement européens de 2014. En outre, nous avons établi 52 rapports annuels spécifiques ${ }^{34}$, notre rapport d'activité 2014, et nous avons présenté à la CONT 2 analyses panoramiques sur l'obligation de rendre compte au sein de l'UE et sur les risques liés à la gestion financière.

Article 163(1) du Règlement (EU, EURATOM) 2015/1929 du Parlement européen et du Conseil du 28 octobre 2015 modifiant la règlementation (EU, Euratom) $N^{\circ} 966 / 2012$ sur les règles financières applicables au budget général de I'Union. 


\section{3) Procédures parlementaires destinées au rapport de l'ISC}

En vertu de la Règle 125 du Règlement intérieur du PE, dans le contexte de la procédure de décharge ou des activités parlementaires liées au contrôle budgétaire, le Président de la Cour des comptes peut être invité à prendre la parole pour présenter les commentaires contenus dans le rapport annuel, les rapports spéciaux ou les avis, ou encore dans le but d'expliquer le programme de travail. Le Parlement peut décider d'organiser un débat séparé sur toute question évoquée dans ces documents, avec la participation de la Commission européenne et du Conseil, en particulier lorsque des irrégularités ont été relevées dans la gestion financière.

La Commission CONT du Parlement est notre partenaire majeur. Comme toutes les autres commissions parlementaires, la CONT élit un président et jusqu'à quatre vice-présidents parmi ses membres titulaires. En règle générale, la structure politique (et la composition) des commissions parlementaires reflète celle de l'assemblée plénière.

En ce qui concerne nos rapports spéciaux, la CONT, par le biais de ses coordinateurs de groupes politiques, nomme un membre rapporteur pour chaque rapport. Ce membre rapporteur a pour mission de préparer un document de travail qui servira de base aux délibérations en commission et qui est souvent utilisé pour préparer la procédure de décharge annuelle. Les débats pléniers et les votes législatifs sont toujours précédés de l'étape dédiée à la commission, et ils s'appuient sur les rapports de la commission.

Pendant les réunions de la commission, un échange de vue sur le rapport spécial de l'ECA et sur le document de travail a lieu, en présence du membre de l'ECA compétent et d'un représentant de la Commission européenne. La commission du PE examine méticuleusement tous les rapports spéciaux de I'ECA parce qu'ils font partie intégrante de la procédure de décharge. Par ailleurs, le Parlement adopte une résolution distincte concernant les rapports spéciaux publiés pendant l'année de la décharge en question. C'est surtout dans le cadre de cette procédure annuelle de décharge que les observations contenues dans nos rapports spéciaux et annuels servent de base à la décision prise par le PE dans le cadre de sa résolution sur la décharge.

Par ailleurs, au sein de la procédure de décharge sur l'exécution du budget, les débats de la CONT suivent la structure des chapitres de notre rapport annuel. Ces débats se déroulent sur plusieurs réunions de la CONT et ils intègrent l'audition des Commissaires responsables des différents domaines budgétaires. Le membre de I'ECA responsable du chapitre en question est également présent. Les Commissaires sont invités à rendre compte au PE de manière détaillée $\mathrm{PE}$ des dépenses budgétaires dans leur domaine respectif. La CONT se prononce sur la décharge budgétaire fin mars et le vote final a lieu en séance plénière en avril.

Les institutions européennes sont tenues d'apporter des réponses aux observations contenues dans la résolution duPE et de prendre des mesures pour préserver les deniers publics en améliorant la qualité des systèmes de gestion et en adoptant les mesures nécessaires à la protection des finances de l'Union. À la demande du PE ou du Conseil, les institutions sont tenues de rendre compte des mesures prises à la lumière de ces observations et commentaires. 


\section{Personnel}

Chaque commission parlementaire est assistée dans l'exercice de ses fonctions par un secrétariat de commission, par les directions sectorielles du PE (fournissant des services de recherche parlementaire et des notes contextuelles) et par les secrétariats des groupes politiques. Le secrétariat de commission facilite les échanges avec les autres institutions et les tiers intéressés, il met en place les dispositifs nécessaires au bon déroulement de l'examen des textes en commission, il conseille les parlementaires sur toutes les questions de procédure et il apporte son assistance dans la rédaction des projets de rapports et de résolutions.

\section{Participation de l'ISC à l'examen de ses rapports par le Parlement}

Concernant les rapports spéciaux, le Membre chargé des relations institutionnelles et l'équipe qui l'appuie tiennent les commissions parlementaires régulièrement informées des rapports spéciaux que nous avons planifiés, et de la date de publication prévue. Cela aide les commissions à planifier leurs activités en lien avec les rapports. Une fois que les rapports sont achevés et approuvés (" adoptés ») par I'ECA, le Membre rapporteur de I'ECA contacte le membre rapporteur de la CONT et lui remet un exemplaire anticipé et confidentiel du rapport spécial. Avant la réunion de la CONT, des communications / réunions sont souvent organisées afin de donner au rapporteur de la CONT de plus amples informations sur les constatations et les recommandations formulées dans le rapport spécial, renseignements utiles en prévision du débat de la commission. À l'invitation de la CONT, le Membre rapporteur de l'ECA présente le rapport spécial à la commission, répond aux questions des MEP et participe à la discussion. Lors de la 8ème législature (2014-2019), la CONT a établi une nouvelle modalité par laquelle elle invite la commission spécialisée compétente à assister à la réunion. Des réunions mixtes sont parfois organisées. Par ailleurs, après avoir été présentés à la CONT, certains de nos rapports spéciaux sont présentés et discutés par les autres commissions spécialisées compétentes qui s'intéressent particulièrement au sujet.

En ce qui concerne le rapport annuel, nous transmettons ce dernier aux institutions de l'UE et le publions au Journal officiel, accompagné des réponses des institutions. Le Président de l'ECA présente les rapports annuels à la CONT et au Parlement réuni en séance plénière, et il prend part au débat portant sur la décharge. Conformément à la procédure de décharge, la discussion des différents chapitres du rapport annuel a lieu sur plusieurs réunions où sont entendus les Commissaires responsables des différents domaines budgétaires, en présence des Membres de l'ECA compétents.

\section{4) Rapports de I'ISC}

Nos rapports spéciaux contiennent des constatations, des conclusions et des recommandations sur le sujet contrôlé. Elles peuvent concerner aussi bien des opérations individuelles que des systèmes de contrôle.

Notre rapport annuel est divisé en chapitres qui traitent des différents domaines budgétaires de l'Union. Chaque chapitre comporte des conclusions et des recommandations sur le domaine en question. Outre l'appréciation portant sur la légalité et la régularité des opérations sous-jacentes, le rapport fournit des observations sur la performance des dépenses dans certains domaines. Par ailleurs, les résultats de notre examen des systèmes de contrôle sélectionnés sont présentés dans les chapitres recettes et dépenses, conjointement avec des recommandations visant à leur amélioration. 


\section{5) Suivi des rapports de l'ISC}

Depuis 2012, l'ECA a réalisé des analyses portant sur le suivi par la Commission des recommandations formulées dans ses rapports spéciaux précédents. Nous avons publié trois rapports récapitulatifs sur le suivi (SR 19/2012, SR 19/2013 et SR 2/2016) et le résumé des observations du suivi de 2013 a été intégré au rapport annuel de 2013. Le suivi des recommandations formulées dans le rapport annuel précédent est réalisé et consigné pour chaque année dans les chapitres concernés.

Les rapports spéciaux examinant le suivi font partie intégrante de la procédure de décharge. En vertu de I'Article 166 du Règlement financier, la Commission et les autres institutions sont tenues de prendre toute mesure appropriée pour donner suite de manière satisfaisante aux observations accompagnant la décision de décharge prise par lePE et aux commentaires joints à la recommandation de décharge adoptée par le Conseil.

\section{6) Audits réalisés à la demande du Parlement}

En vertu de l'Article 287 TFUE, la Cour des comptes doit fournir au Parlement et au Conseil une déclaration d'assurance concernant la fiabilité des comptes ainsi que la légalité et la régularité des opérations sous-jacentes. Elle est tenue d'établir un rapport annuel après la clôture de l'exercice budgétaire.

En ce qui concerne les rapports spéciaux, le Traité stipule que l'ECA peut, en outre, présenter à tout moment ses observations, notamment sous forme de rapports spéciaux, sur des questions particulières. II n'y a pas de disposition juridique permettant au Parlement de demander des audits sur des sujets particuliers, bien que nous prenions en compte ses suggestions et ses priorités lorsque nous déterminons notre programme de travail. Voir également les réponses aux questions 7 et 9 ci-dessous.

\section{7) Programme de travail}

Les suggestions et les priorités du PE nous sont connues par différentes sources : documents de décharge, compte-rendus des réunions des commissions, consultations avec la Conférence des présidents de commissions ${ }^{35}$, présentation de notre programme de travail ${ }^{36}$, échanges individuels avec des parlementaires, etc. Nous tenons compte de toutes ces suggestions, et de nos autres priorités, lorsque nous déterminons notre programme de travail, et en particulier les sujets des audits sélectionnés.

\section{8) Niveau et fréquence des échanges}

Le Président et les membres de l'ECA entretiennent des échanges réguliers avec les commissions parlementaires, en particulier avec la CONT. Tous les ans, le Président de l'ECA participe aux réunions de la CONT afin de présenter notre programme de travail et nos rapports annuels, ainsi qu'aux séances plénières du Parlement pour présenter les rapports annuels et prendre part au débat sur la décharge. Il a

La Conférence des présidents de commissions (CCC) est une instance politique qui coordonne les travaux des commissions et veille à ce qu'il y ait une bonne coopération entre elles. Elle comprend les présidents de toutes les commissions permanentes et restreintes. Elle se réunit une fois par mois. 
en outre des échanges réguliers avec la Conférence des présidents de commissions comme cela été décrit ci-dessus.

De plus, la coopération entre la CONT, ses membres et le Membre de I'ECA chargé des relations institutionnelles a été développée, approfondie et améliorée depuis la nomination de ce dernier en avril 2014, contribuant ainsi à identifier des opportunités pour présenter nos travaux et accroitre leur utilisation et, par conséquent, pour renforcer leur impact.

Au sein de la Direction de la Présidence, une équipe dédiée aux relations avec les institutions a été créée pour coordonner, consolider et renforcer les relations avec la CONT et les autres commissions parlementaires. Cette équipe facilite les échanges quotidiens avec le secrétariat de la CONT et augmente la fréquence des contacts avec les secrétariats des autres commissions. L'équipe coordonne et assiste à toutes les réunions de la CONT et des commissions parlementaires concernées, et elle fournit rapidement des informations en retour aux Membres et à la direction de l'ECA.

Tous les ans, les parlementaires de la CONT et le personnel de son secrétariat tiennent une réunion avec les Membres de l'ECA et du personnel d'appui pour discuter des questions d'intérêt commun dans le but d'améliorer la coopération.

\section{9) Bonnes pratiques}

Quelques exemples:

- Nomination d'un membre de l'ECA chargé des relations avec les institutions ; cette initiative a contribué à renforcer nos relations avec les autres institutions de l'Union au niveau stratégique et à identifier les possibilités d'accroître l'influence de nos travaux d'audit ;

- Incitation des commissions parlementaires à nous suggérer des domaines et thèmes prioritaires à prendre en compte lorsque nous établissons notre programme de travail ;

- Coopération de I'ECA avec les autres organes parlementaires (Direction générale Politiques internes et Service de recherche parlementaire européen), qui apporte à un public plus large nos rapports spéciaux et améliore leur utilisation. Cette initiative vise par ailleurs à créer un réseau d'échange d'informations, et à offrir aux deux parties des informations précieuses concernant l'évaluation des politiques et des risques, l'évaluation de la performance, l'évaluation de la valeur ajoutée et de l'impact ;

- Création d'une base de données et d'un système de gestion puissants, pour organiser (envoyer et enregistrer) nos communications avec les parties prenantes, nous permettant ainsi de cibler l'envoi de nos rapports et nos informations d'audit aux personnes et aux institutions en fonction de leurs intérêts spécifiques.

\section{0) Perspective}

Depuis trois ans, notre institution a consacré beaucoup de temps et d'efforts au développement de la coopération avec le Parlement européen et pour y renforcer sa présence. Cette action est le résultat de la stratégie globale de communication et de relation avec les parties prenantes que nous avons préparée en 2013. À l'avenir, nous allons nous efforcer de consolider notre présence au sein des commissions spécialisées, de mieux connaître les attentes du Parlement et d'améliorer notre réactivité à ses demandes. Nous accomplirons ces tâches tout en préservant notre indépendance en qualité d'institution de contrôle externe de l'UE. 
FRANCE

Cour des comptes

\section{1) Cadre juridique}

Le cadre juridique est essentiellement défini par :

- La Section 47-2 de la Constitution française, votée en juillet 2008 : " La Cour des Comptes assiste le Parlement dans le contrôle de l'action du Gouvernement. Elle assiste le Gouvernement et le Parlement dans le contrôle de l'exécution des lois de finances et de l'application des lois de financement de la sécurité sociale ainsi que dans l'évaluation des politiques publiques. Par ses rapports publics, elle contribue à l'information des citoyens. "

- La loi organique relative aux lois de finances votée en août 2001, particulièrement la Section 58 déterminant la liste des rapports déposés par la Cour des comptes devant le Parlement, à sa demande ou annuellement.

- Le Code des Juridictions Financières qui définit les relations entre les Chambres parlementaires et la Cour des comptes.

\section{2) Modalités de soumission des rapports de l'ISC au parlement}

Deux types de rapports sont envoyés au Parlement : des rapports réguliers et des audits sur demande.

\section{Rapports réguliers comportant des dates butoir}

La Cour des comptes fournit au Parlement :

- Son rapport annuel (remis au Président de l'Assemblée nationale), remis également au Président de la République ;

- Le rapport sur l'exécution de la Loi de financement de la sécurité sociale (au Président de chacune des deux Chambres);

- Le rapport annuel sur les finances publiques locales (au Président de chacune des deux Chambres);

- L'audit annuel sur les états financiers de l'État et sur ceux de la sécurité sociale (présidents des commissions permanentes des finances et des affaires sociales) ;

- Le rapport sur l'équilibre budgétaire et sur l'application des lois de finances (présidents des commissions permanentes des finances) ;

- Le rapport sur la situation et les perspectives des finances publiques (présidents des commissions permanentes des finances).

Le calendrier de dépôt est déterminé par les lois et les lois organiques et il vise à donner aux parlementaires les informations, les outils et les analyses nécessaires à l'examen des projets de loi de finances.

Les rapports publics thématiques de la Cour (plus de 20 par an) et les référés adressés au Gouvernement sur toute politique publique sont également transmis au Parlement. 


\section{Rapports sur demande}

La loi organique relative aux lois de finances d'août 2001 permet aux Commissions permanentes des finances des deux Chambres (I'Assemblée nationale et le Sénat) de demander des rapports sur un thème particulier. La Cour des comptes a neuf mois pour leur envoyer le rapport. Ce délai peut être prolongé en accord avec la commission permanente concernée. La Cour des comptes transmet à chaque Chambre cinq rapports par an.

La Commission permanente des affaires sociales peut également demander des rapports. La Cour des comptes en envoie environ un à chacune des Chambres par an.

Les rapports peuvent porter sur n'importe quel sujet lié aux politiques publiques. Ils sont toujours adressés au président de la commission permanente à l'origine de la demande.

\section{Procédures d'envoi des rapports au Parlement}

Chaque audit réalisé sur demande est présenté par la Cour des comptes pendant une audition publique devant la commission permanente.

Le rapport annuel est présenté devant l'Assemblée par le Premier Président de la Cour. 
Nombre et type de rapports

\begin{tabular}{|c|c|c|c|c|c|c|c|}
\hline & $\begin{array}{l}\text { Rapports } \\
\text { d'audit } \\
\text { financier }\end{array}$ & $\begin{array}{c}\text { Rapports d'audit } \\
\text { de conformité }\end{array}$ & $\begin{array}{l}\text { Rapports d'audit } \\
\text { de performance }\end{array}$ & $\begin{array}{c}\text { Autres rapports } \\
\text { ou documents }\end{array}$ & $\begin{array}{l}\text { Rapports } \\
\text { d'activité } \\
\text { annuels }\end{array}$ & $\begin{array}{c}\text { Rapports } \\
\text { demandés par } \\
\text { le Parlement }\end{array}$ & Remarques \\
\hline $\begin{array}{c}\text { Nombre de } \\
\text { rapports soumis }\end{array}$ & 1 & 2 & 4 & & 1 & 15 & \\
\hline $\begin{array}{c}\text { Nombre de } \\
\text { rapports discutés } \\
\text { par le Parlement }\end{array}$ & 1 & 2 & 4 & & 1 & 15 & \\
\hline $\begin{array}{l}\text { Soumis au } \\
\text { Président }\end{array}$ & 0 & 0 & 0 & & 0 & $\mathrm{~N}$ & \\
\hline $\begin{array}{l}\text { Soumis au } \\
\text { Président de la } \\
\text { commission } \\
\text { spécialisée }\end{array}$ & 0 & 0 & 0 & & 0 & 0 & \\
\hline $\begin{array}{c}\text { Copies envoyées } \\
\text { aux autres } \\
\text { commissions } \\
\text { concernées }\end{array}$ & 0 & 0 & 0 & & 0 & $\mathrm{~N}$ & \\
\hline $\begin{array}{c}\text { Notes de synthèse } \\
\text { destinées à la } \\
\text { commission } \\
\text { parlementaire }\end{array}$ & 0 & 0 & 0 & & 0 & 0 & \\
\hline $\begin{array}{c}\text { Résumés soumis au } \\
\text { Parlement }\end{array}$ & 0 & 0 & 0 & & 0 & 0 & \\
\hline
\end{tabular}

3) Procédures parlementaires utilisées dans le traitement des rapports de l'ISC

Les rapports sont négociés entre la Cour et la commission permanente, particulièrement la détermination du sujet et du calendrier.

La Commission permanente des finances et la Commission permanente des affaires sociales procèdent à des demandes d'audit. Le Président de la Commission permanente des finances est un membre de l'opposition, à l'Assemblée nationale depuis 2007, et au Sénat depuis 2012. Ce principe est fondé sur la tradition.

Pour chaque rapport, la commission nomme toujours un rapporteur.

Une audition préliminaire a lieu entre le rapporteur et le personnel chargé du rapport. Une audition publique dédiée à la présentation du rapport a lieu lorsque le rapport est déposé devant la commission.

La commission n'a jamais eu recours à des experts externes indépendants dans l'examen des rapports. 
Le débat sur les rapports de l'ISC a lieu en commission, quelquefois en séance plénière, au moins une fois par an en ce qui concerne le rapport annuel.

D'autres commissions peuvent également analyser les rapports de l'ISC, même si le thème du rapport est souvent très spécifique.

La commission autorise la publication du rapport par un vote à la fin de l'audition. II n'y a pas de procédure de décharge formalisée.

\section{Personnel}

Chaque commission chargée du contrôle budgétaire dispose environ de quinze agents, et chaque commission des affaires sociales en compte dix. Ils sont chargés de toutes les tâches répertoriées cidessous. Ils ont le même grade que celui des agents de I'ISC.

\section{Participation de I'ISC à l'examen de ses rapports par le Parlement}

Le niveau de représentation de la Cour aux auditions est très élevé (Président ou Premier Président). Le représentant fait un discours puis les parlementaires lui posent des questions.

L'ISC fait une présentation officielle devant la commission, et une fois par an (rapport annuel) devant l'Assemblée au complet, mais le Parlement est libre de poser n'importe quelle question relative au rapport. Le rôle de I'ISC est de fournir le rapport et de répondre aux questions soulevées par les parlementaires. L'ISC ne prépare pas de questions lorsqu'un membre du gouvernement est auditionné, même si l'audition s'appuie sur son rapport.

\section{4) Rapports de l'ISC}

Dans sa communication avec le Parlement, I'ISC se concentre sur des problèmes génériques / systémiques et sur la formulation de recommandations.

La configuration et le fonctionnement des systèmes de contrôle internes n'est pas un sujet mis en exergue dans les rapports de I'ISC, car le contrôle interne n'intéresse généralement pas les parlementaires.

Tous les rapports d'audit contiennent des conclusions et des opinions claires.

\section{5) Suivi des rapports de I'ISC}

Cette question ne concerne pas uniquement le Parlement.

La Cour assure elle-même un suivi très précis des recommandations formulées dans n'importe quel type de rapports, y compris dans les rapports destinés au Parlement. Le rapport public annuel analyse les résultats des opérations de suivi. Ces informations sont régulièrement utilisées par les parlementaires, notamment lors du débat budgétaire.

\section{6) Audits réalisés à la demande du Parlement}

La loi organique relative aux lois de finances d'août 2001 permet aux commissions permanentes des finances des deux Chambres (I'Assemblée nationale et le Sénat) de demander des rapports sur un thème particulier. La Cour des comptes a neuf mois pour leur envoyer son rapport. Ce délai peut être prolongé 
en accord avec la commission permanente concernée. La Cour des comptes transmet à chaque Chambre cinq rapports par an.

La Commission permanente des affaires sociales peut également demander des rapports. La Cour des comptes en envoie environ un par an à chaque Chambre.

\section{7) Programme de travail}

La Cour des comptes étant une juridiction, le Conseil constitutionnel ne l'autorise pas à transmettre son programme de travail au Parlement. En conséquence, le programme de la Cour des comptes n'est pas soumis à des pressions ou à des suggestions du Parlement.

\section{8) Niveau et fréquence des échanges}

Il est véritablement impossible de préciser la fréquence des échanges, en particulier en ce qui concerne les échanges informels. Le Parlement organise entre 40 et 50 auditions annuelles, publiques ou non. Une personne est responsable des relations entre la Cour et le Parlement, mais pas à temps plein.

\section{9) Bonnes pratiques}

L'impact des rapports de la Cour dépend uniquement de la volonté des parlementaires de mettre en œuvre les recommandations préconisées par la Cour. En conséquence, la Cour doit tout particulièrement veiller à ce que les rapports soient rédigés avec clarté, et que les recommandations puissent être appliquées par le Parlement. Le personnel est conscient de cet impératif car le suivi des recommandations fait partie de leurs fonctions.

\section{0) Perspective}

Les relations sont établies par des lois assez récentes, que la Cour aussi bien que le Parlement ont mis du temps à assimiler. Un ancien Premier Président de la Cour, qui était également un ancien Président de l'Assemblée nationale, disait que la Cour devrait se situer " juste entre le Parlement et le Gouvernement. »

Les relations sont donc excellentes et elles ne sont pas appelées à évoluer à court terme car le statut de juridiction de la Cour n'autorise pas davantage de rapprochement. 


\section{L'ANCIENNE RÉPUBLIQUE YOUGOSLAVE DE MACÉDOINE}

Bureau national d'audit

\section{1) Cadre juridique}

La communication avec le Parlement est réglementée par la Loi relative au Bureau national d'audit (SAL), d'après laquelle le rapport annuel et les rapports d'audit finaux du Bureau national d'audit (SAO) sont envoyés au Parlement. Le programme de travail du SAO est soumis à l'examen du Parlement. Dans les lignes directrices du SAO proposant des audits pour son programme annuel (une législation interne au $\mathrm{SAO})$, ce dernier peut intégrer les audits proposés par le Parlement.

\section{2) Modalités de soumission des rapports de l'ISC au parlement}

Le SAO dépose devant le Parlement tous les rapports d'audit finaux, quelle que soit leur nature (audit de régularité ou audit de performance). Les rapports sont adressés au Président du Parlement de la République de Macédoine.

Article 33 (de la SAL) :

1. Le Bureau national d'audit établit un rapport annuel sur les audits qu'il a exécutés ainsi que sur son fonctionnement.

2. Le Bureau national d'audit soumet le rapport cité à l'alinéa (1) du présent article à l'examen du Parlement de la République de Macédoine au plus tard le 30 juin pour l'année antérieure.

3. Le Parlement de la République de Macédoine examine le rapport cité à l'alinéa (1) du présent article et formule ses conclusions.

4. Les rapports d'audit finaux visés à l'Article 30 alinéa (3) de la présente loi sont soumis au Parlement de la République de Macédoine.

\section{Procédures d'envoi des rapports au Parlement}

L'ISC dépose devant le Parlement tous les rapports d'audit finaux. Une copie du rapport annuel du SAO est envoyée à chaque parlementaire. L'Auditeur général (AG) assiste à l'examen du rapport annuel par la Commission parlementaire du budget et des finances et fournit des explications à ces fins. La même procédure s'applique à la séance plénière du Parlement qui examine le rapport annuel et adopte des conclusions. 
Nombre et type de rapports

\begin{tabular}{|c|c|c|c|c|c|c|c|}
\hline & $\begin{array}{l}\text { Rapports } \\
\text { d'audit } \\
\text { financier }\end{array}$ & $\begin{array}{l}\text { Rapports d'audit } \\
\text { de conformité }\end{array}$ & $\begin{array}{l}\text { Rapports d'audit } \\
\text { de performance }\end{array}$ & $\begin{array}{c}\text { Autres rapports } \\
\text { ou documents }\end{array}$ & $\begin{array}{l}\text { Rapports } \\
\text { d'activité } \\
\text { annuels }\end{array}$ & $\begin{array}{c}\text { Rapports } \\
\text { demandés par } \\
\text { le Parlement }\end{array}$ & Remarques \\
\hline $\begin{array}{c}\text { Nombre de } \\
\text { rapports soumis }\end{array}$ & $43^{*}$ & $43^{*}$ & $18+13^{*}$ & 1 & 1 & & \\
\hline $\begin{array}{c}\text { Nombre de } \\
\text { rapports discutés } \\
\text { par le Parlement }\end{array}$ & & & & & 1 & & \\
\hline $\begin{array}{l}\text { Soumis au } \\
\text { Président }\end{array}$ & 0 & 0 & 0 & 0 & 0 & & \\
\hline $\begin{array}{l}\text { Soumis au } \\
\text { Président de la } \\
\text { commission } \\
\text { spécialisée }\end{array}$ & $\mathrm{N}$ & $\mathrm{N}$ & $\mathrm{N}$ & $\mathrm{N}$ & $\mathrm{N}$ & & \\
\hline $\begin{array}{c}\text { Copies envoyées } \\
\text { aux autres } \\
\text { commissions } \\
\text { concernées }\end{array}$ & $\mathrm{N}$ & $\mathrm{N}$ & $\mathrm{N}$ & $\mathrm{N}$ & $\mathrm{N}$ & & \\
\hline $\begin{array}{c}\text { Notes de synthèse } \\
\text { destinées à la } \\
\text { commission } \\
\text { parlementaire }\end{array}$ & $\mathrm{N}$ & $\mathrm{N}$ & $\mathrm{N}$ & $\mathrm{N}$ & $\mathrm{N}$ & & \\
\hline $\begin{array}{c}\text { Résumés soumis au } \\
\text { Parlement }\end{array}$ & $\mathrm{N}$ & $\mathrm{N}$ & $\mathrm{N}$ & $N$ & $\mathrm{~N}$ & & \\
\hline
\end{tabular}

43* Tous les audits financiers comprennent un audit de conformité.

13* Ces pièces sont des audits de performance liés aux audits internes sur la gestion et la vérification financière, ainsi que leur harmonisation.

\section{3) Procédures parlementaires utilisées dans le traitement des rapports de l'ISC}

Le Parlement ne dispose pas de commission dédiée à l'examen des rapports du SAO, et la Commission du budget et des finances est désignée à ces fins. Elle est habituellement présidée par un membre du parti d'opposition et réglementée par une procédure.

Dans la pratique, les rapports d'audit individuels ne sont pas discutés au Parlement.

En juin 2013, le Bureau national d'audit a signé un protocole de coopération avec le Parlement de la République de Macédoine qui réglemente les domaines et les méthodes de coopération entre les deux parties. 


\section{Personnel}

La Commission du budget et des finances dispose d'un effectif restreint. Le rôle de son personnel est défini par le Règlement intérieur du Parlement et par d'autres textes parlementaires. En conséquence, il est impossible au SAO de répondre plus précisément à cette question. Le SAO a autorisé son personnel à communiquer avec le Parlement et le Parlement a fait de même, ce qui leur permet de communiquer si nécessaire.

\section{Participation de I'ISC à l'examen de ses rapports par le Parlement}

L'AG assiste aux séances plénières du Parlement lors des délibérations sur le rapport annuel du SAO. L'AG procède à une brève présentation du rapport annuel et il répond aux questions qui lui sont posées. En séance plénière, les problématiques énoncées dans les différents rapports d'audit soumis au Parlement peuvent également faire l'objet d'un débat, auquel cas l'AG apporte les explications appropriées.

\section{4) Rapports de I'ISC}

Dans le rapport annuel adressé au Parlement, le SAO se concentre sur des problèmes et des recommandations génériques / systémiques.

Le SAO considère que la configuration et le fonctionnement des systèmes de contrôle internes revêtent une grande importance, c'est pourquoi il met ces sujets en avant dans ses rapports d'audit. Dans ce domaine, en 2015, le SAO a réalisé 13 audits de performance sur la gestion et le contrôle financiers, sur les audits internes et leur harmonisation.

Tous les rapports d'audit contiennent des conclusions, des recommandations et des opinions claires.

\section{5) Suivi des rapports de l'ISC}

Le SAO conserve une trace des conclusions et des recommandations acceptées par l'entité contrôlée en utilisant le logiciel SAPRI. Les audits et les bilans de suivi sont exécutés au début de chaque année calendaire afin de constater le niveau de mise en œuvre des recommandations contenues dans les rapports d'audit finaux de l'année antérieure.

Le gouvernement macédonien est tenu de rendre compte de la mise en œuvre des recommandations du SAO.

\section{6) Audits réalisés à la demande du Parlement}

Le Parlement peut demander un audit, conformément aux lignes directrices relatives à l'intégration d'audits dans le programme annuel du SAO (loi interne du SAO). À ce jour, le Parlement n'a demandé aucun audit.

\section{7) Programme de travail}

Le SAO est indépendant dans son choix des entités à contrôler.

\section{8) Niveau et fréquence des échanges}

Des échanges ont lieu entre l'AG et le Président du Parlement, et l'AG et le Président de la Commission parlementaire du budget et des finances, notamment durant la phase préparatoire de la séance plénière ayant le rapport annuel du SAO à l'ordre du jour. 
Les échanges entre le SAO et le Parlement ont souvent lieu par l'intermédiaire de leur personnel chargé de la communication.

\section{9) Bonnes pratiques}

Le SAO est toujours prêt à améliorer sa coopération avec le Parlement. Un travail de collaboration avec la Cour des comptes néerlandaise a produit le manuel intitulé " Introduction aux rapports d'audit ", offert aux parlementaires pour faciliter la compréhension des rapports d'audit.

\section{0) Perspective}

Le Parlement devrait créer des instances qui lui permettraient d'examiner les rapports d'audit (création d'une commission spéciale ou d'une commission au sein des commissions parlementaires existantes) en employant des experts qualifiés en nombre suffisant qui seraient en mesure de comprendre et d'analyser les rapports d'audit. Le SAO est toujours prêt à fournir l'assistance nécessaire en organisant des séances d'informations et de travail. 


\section{ALLEMAGNE}

Cour des comptes fédérale

\section{1) Cadre juridique}

L'Article 114.2 de la Constitution allemande (Loi fondamentale) garantit l'existence de I'ISC allemande et l'indépendance juridictionnelle de ses membres. Elle détermine le périmètre de ses contrôles sur le budget et les comptes de capitaux, la gestion financière fédérale et l'exécution du budget, et fixe les critères de régularité, de conformité et de performance applicables aux audits. Elle impose en outre à I'ISC allemande de rendre compte aux deux chambres du Parlement et au gouvernement fédéral au moins une fois par an.

La Loi relative au Bundesrechnungshof contient des dispositions détaillées qui définissent le statut de I'ISC, institution autonome ayant pour mission de contrôler le gouvernement et soumise uniquement à la loi, au statut de ses membres et aux procédures décisionnelles collégiales.

Des dispositions supplémentaires établissant les fonctions de I'ISC, les thèmes, les critères et les procédures d'audit, l'établissement de rapports et le principe de bonne gouvernance, sont stipulées dans les réglementations financières fédérales, dans notre loi fédérale de finances (Articles. 88 et suivants) et dans la Loi relative aux principes budgétaires (Articles. 54 et suivants).

\section{2) Modalités de soumission des rapports de l'ISC au parlement}

Les rapports d'audit de I'ISC allemande ne font pas de distinction entre les rapports d'audit financier, d'audit de conformité et d'audit de performance. Ces rapports sont habituellement des documents complets traitant tous les aspects des contrôles, en accordant toutefois une place particulière aux audits de performance.

Chaque année, l'ISC allemande exécute environ 1300 audits et rapporte les constatations pertinentes aux organismes contrôlés, à savoir principalement les départements (ministères) du gouvernement fédéral, sous la forme de lettres d'audit, en leur donnant la possibilité de commenter ces rapports préliminaires. Un rapport annuel reprend les constatations majeures et les recommandations formulées pendant ces audits, puis il est envoyé aux deux assemblées parlementaires et au gouvernement fédéral (Article 97.1 de la Loi fédérale de finances) le dernier trimestre de l'année.

Le rapport annuel comprend une section finale qui explique comment I'ISC doit exécuter son mandat. L'ISC allemande peut également à tout moment publier des rapports spéciaux afin d'informer les organes législatifs et le gouvernement sur des questions particulièrement importantes (Article 99 de la Loi fédérale des finances). Le rapport annuel et les rapports spéciaux sont publiés en tant que documents parlementaires et traités essentiellement par la Commission du contrôle budgétaire (PAC) de la Commission du budget du Bundestag (I'assemblée directement élue du Parlement allemand). La PAC accepte la majorité des conclusions et des recommandations de l'ISC.

Les constatations, les conclusions et les recommandations formulées dans chaque audit sont reprises dans une lettre de recommandation. Le Président de I'ISC allemande exerce une fonction ex officio en tant que Commissaire à la performance fédérale. À ce titre, il peut réaliser des audits dont les résultats sont publiés dans un ensemble d'opinions d'audit. Ce type d'audit suit souvent une approche horizontale. 
L'ISC allemande assure une mission de conseil. Elle peut publier des rapports consultatifs qui abordent des problématiques de son choix et qui fournissent des conseils au gouvernement et au Parlement sur des thématiques particulières. L'ISC peut en outre jouer un rôle consultatif au regard du cycle budgétaire.

\section{Procédures d'envoie des rapports au Parlement}

L'ISC allemande fournit des éléments d'information à la PAC sur ces constatations d'audit afin d'obtenir le soutien de la PAC.

Elle peut publier des rapports consultatifs qui abordent des problématiques de son choix et qui fournissent des conseils au gouvernement et au Parlement sur des thématiques particulières. L'ISC peut en outre jouer un rôle consultatif au regard du cycle budgétaire.

Avant la publication du rapport final, l'ensemble des faits et des chiffres est soumis à un processus de vérification. L'entité contrôlée reçoit le rapport d'audit provisoire et peut y apporter ses commentaires. Ainsi, toute controverse découlant des constations d'audit peut être préalablement aplanie. 
Nombre et type de rapports

\begin{tabular}{|c|c|c|c|c|c|c|c|}
\hline & $\begin{array}{l}\text { Rapports } \\
\text { d'audit } \\
\text { financier }\end{array}$ & $\begin{array}{c}\text { Rapports d'audit } \\
\text { de conformité }\end{array}$ & $\begin{array}{l}\text { Rapports d'audit } \\
\text { de performance }\end{array}$ & $\begin{array}{c}\text { Autres rapports } \\
\text { ou documents }\end{array}$ & $\begin{array}{l}\text { Rapports } \\
\text { d'activité } \\
\text { annuels }\end{array}$ & $\begin{array}{c}\text { Rapports } \\
\text { demandés par } \\
\text { le Parlement }\end{array}$ & Remarques \\
\hline $\begin{array}{c}\text { Nombre de } \\
\text { rapports soumis }\end{array}$ & & & & & & & \\
\hline $\begin{array}{c}\text { Nombre de } \\
\text { rapports discutés } \\
\text { par le Parlement }\end{array}$ & & & & & & & \\
\hline $\begin{array}{l}\text { Soumis au } \\
\text { Président }\end{array}$ & 0 & 0 & 0 & 0 & 0 & 0 & \\
\hline $\begin{array}{l}\text { Soumis au } \\
\text { Président de la } \\
\text { commission } \\
\text { spécialisée }\end{array}$ & 0 & 0 & 0 & 0 & 0 & 0 & \\
\hline $\begin{array}{c}\text { Copies envoyées } \\
\text { aux autres } \\
\text { commissions } \\
\text { concernées }\end{array}$ & & & & & & & \\
\hline $\begin{array}{l}\text { Notes de synthèse } \\
\text { destinées à la } \\
\text { commission } \\
\text { parlementaire }\end{array}$ & 0 & 0 & 0 & 0 & 0 & 0 & \\
\hline $\begin{array}{c}\text { Résumés soumis au } \\
\text { Parlement }\end{array}$ & & & & & & & \\
\hline
\end{tabular}

* Chaque rapport annuel et chaque rapport spécial est précédé d'un résumé.

3) Procédures parlementaires utilisées dans le traitement des rapports de I'ISC

Le rapport annuel destiné au Parlement est adopté par le Conseil de I'ISC allemande puis transmis par le Président de I'ISC aux Présidents des deux chambres parlementaires et au gouvernement fédéral.

Le Bundestag (la chambre parlementaire élue au suffrage direct) transmet le rapport annuel à la Commission budgétaire du Parlement qui le remet à son tour à la PAC.

La PAC désigne des rapporteurs et détermine le programme de discussion du rapport annuel.

La PAC délibère sur les différents points du rapport annuel puis vote des résolutions demandant aux ministères de prendre des mesures visant la mise en œuvre des recommandations de I'ISC, qui a ensuite six mois pour faire état des actions prises par le ministère. Pour terminer, la PAC évalue le résultat global de ses discussions et décide de recommander des mesures en lien avec le mécanisme de décharge budgétaire. Le rapport et la résolution provisoire de la PAC sont votés au Bundestag réuni en séance 
plénière. Ce rapport permet de donner décharge au gouvernement fédéral de sa gestion budgétaire (Article 114 alinéa 1 Constitution allemande, Article 114 Loi fédérale de finances).

\section{Personnel}

La Commission du budget compte 41 membres. Ils discutent et soumettent une résolution sur la Loi de finances et le budget annuels (budget de I'ISC également) et une proposition de résolution concernant I'octroi de la décharge (fondée sur les délibérations de la PAC) ; ils débattent en outre de tous les projets ayant une incidence sur le budget, ils discutent des dépenses excessives et extraordinaires et du déblocage des fonds bloqués. Ils sont assistés par des rapporteurs (un rapporteur principal et quatre corapporteurs).

La PAC est une commission permanente comprenant 17 membres. Tous ses membres siègent également à la Commission du budget. La composition de la PAC reflète la force des groupes parlementaires. Elle discute et prend des décisions sur toutes les problématiques mises à jour dans les audits. Le rapport annuel de I'ISC sert de base à l'octroi de la décharge au gouvernement fédéral et aux autres rapports de I'ISC. La PAC délibère en appliquant la procédure du rapporteur, selon laquelle certains parlementaires sont responsables de certains ministères ou de domaines thématiques de l'exécutif ; elle prépare en outre les décisions qui seront prises par la PAC au complet. Elle se réunit entre 8 et 10 fois par an, avec un ordre du jour fixé en séance parlementaire. Assistent aux discussions un représentant permanent du Ministère des finances et un de I'ISC, un du ministère sectoriel, ainsi que l'équipe de l'ISC responsable du rapport.

Tous les parlementaires et les commissions parlementaires peuvent s'appuyer sur le soutien des services de recherche et de documentation du Bundestag. Les informations relatives aux effectifs de la Commission du budget du Bundestag ne sont disponibles qu'auprès de l'administration du Bundestag.

\section{Participation de I'ISC à l'examen de ses rapports par le Parlement}

Les recommandations d'audit sont discutées par la PAC et généralement, la majorité est reprise par la Commission.

Le Bundestag et surtout la Commission du budget et la PAC s'appuient sur les conseils expert de l'ISC, notamment dans le cadre des projets et programmes gouvernementaux majeurs qui présentent un risque élevé en termes de bilan coût/bénéfice. Sont toujours présents aux réunions de la PAC les membres compétents de I'ISC à qui la PAC donne habituellement l'opportunité de s'exprimer sur les problématiques débattues. Les membres de I'ISC informent également la Commission du budget et la PAC. Ils leur font également des présentations d'information pendant la phase préparatoire du budget et, si nécessaire, ils interviennent auprès d'autres commissions parlementaires.

\section{4) Rapports de I'ISC}

L'ISC peut sélectionner les travaux d'audit qu'elle juge nécessaire. Tous les thèmes mentionnés ci-dessus peuvent faire l'objet de rapports d'audit. En principe, tous les rapports d'audit comportent des conclusions et des recommandations.

\section{5) Suivi des rapports de l'ISC}

Pour renforcer l'impact des contrôles, I'ISC assure le suivi de ses recommandations d'audit. Elle demande à l'organisme contrôlé quelle action il a pris en réponse aux recommandations ainsi que, si nécessaire, 
des justificatifs probants. Les constatations faites peuvent justifier la soumission d'un rapport au Parlement ou la réalisation d'un audit de suivi. L'équipe composée de membres de I'ISC décide de la conduite, de la nature et du calendrier de l'action de suivi. En outre, comme il l'a été mentionné plus haut, I'ISC est soutenue par la PAC qui peut définir une période de réalisation des recommandations. L'ISC joue en outre le rôle de conseiller expert dans le cadre de la procédure de préparation du budget.

\section{6) Audits réalisés à la demande du Parlement}

Vu la disposition constitutionnelle déterminant l'indépendance de I'ISC, le Parlement peut demander, mais non ordonner, à I'ISC de contrôler toute problématique particulière et de consigner les résultats du contrôle dans un rapport. L'ISC satisfait normalement à ces demandes, non seulement par courtoisie mais parce que le Parlement est souvent son allié en ce qu'il pousse l'entité contrôlée à prendre des mesures correctives en réponse aux conclusions et aux recommandations d'audit. Les demandes d'audit sollicitées par le Parlement ne seraient probablement refusées que si les conclusions et les recommandations risquaient de devenir une "partie de foot politique " ou s'il semblait que la demande allait utiliser des ressources de I'ISC qui devraient être consacrées à des actions plus urgentes.

\section{7) Programme de travail}

L'ISC élabore son programme de travail en toute indépendance. Elle a toute la liberté de sélectionner les thèmes de contrôle qu'elle juge appropriés. Toutefois, elle peut prendre en compte les requêtes parlementaires ainsi que les débats en cours ou imminents concernant des sujets importants.

\section{8) Niveau et fréquence des échanges}

II y a régulièrement des échanges formels et informels entre les membres de I'ISC, de la Commission du budget, de la PAC et, si nécessaire, ceux d'autres commissions parlementaires, notamment par les échanges entre des représentants de l'ISC et les rapporteurs des commissions.

Notre panel (des membres de I'ISC) se présente à " son " rapporteur et lui propose son aide. Les nouveaux présidents et représentants des équipes de travail des différents groupes parlementaires sont contactés personnellement par notre Président ou Vice-Président.

\section{9) Bonnes pratiques}

L'ISC se montre très active dans sa participation aux discussions parlementaires et interministérielles qui se tiennent pendant la phase préparatoire du budget.

L'ISC peut en outre réaliser des audits en temps réel sur des programmes de dépenses tels que la construction d'une route. L'ISC accompagne ces projets et contrôle chaque phase du projet. Ainsi, I'ISC peut contribuer à faire des économies pendant la phase de réalisation du programme.

\section{0) Perspective}

L'ISC n'a pas connaissance de point faible.

11) Y-a-t-il d'autres éléments concernant les relations actuelles entre l'ISC et le Parlement que vous souhaiteriez souligner, et quelles a été leur évolution au cours des cinq dernières années?

Actuellement, l'ISC ne voit rien à relever. 


\section{GRÈCE}

Cour des comptes grecque

\section{1) Cadre juridique}

Le cadre juridique définissant les obligations de la Cour envers le Parlement grec, selon les termes de la Constitution, de la Loi codifiée relative à la Cour des comptes grecque (L. 4129/2013) et la Loi budgétaire organique (L. 4270/2014) prévoit deux rapports :

Le rapport annuel des constations, qui présente les résultats des activités de la Cour, ses observations et ses suggestions destinées à améliorer les systèmes et la législation.

La déclaration annuelle, y compris l'opinion de la Cour sur l'état financier annuel et sur le bilan de l'État.

\section{2) Modalités de soumission des rapports de l'ISC au parlement}

Après avoir examiné les rapports des Commissaires de la Cour basés sur les informations obtenues dans le cadre de leur fonction, la Cour des comptes grecque (HCA) réunie en séance plénière élabore le rapport annuel, dont le contenu est le suivant :

- Rapport reprenant les résultats et les observations des contrôles ;

- Remarques sur les violations relevées des règles administratives, financières ou budgétaires ;

- Suggestions des mesures à prendre pour éviter que les violations ne se répètent ;

- Suggestions des mesures à prendre pour réformer et améliorer la législation portant sur la juridiction de l'HCA.

Ce rapport est transmis aux entités contrôlées (les ministres) par le Ministre des Finances. Les commentaires de ces entités sont transmis dans une publication conjointe à l'HCA par le Ministre des Finances dans un délai de deux mois.

Le rapport annuel se compose de manière détaillée des parties suivantes :

Partie I: $\quad$ Courte description des activités de l'HCA pour l'année concernée (audits, décisions judiciaires, participation aux congrès internationaux, etc.) et synthèses des constatations des audits spéciaux.

Partie II : $\quad$ Observations générales sur les dépenses publiques.

Partie III : $\quad$ Analyse des constatations d'audit les plus significatives (en fonction du seuil précisé pour l'année concernée). Ces constatations documentent les observations formulées dans la partie II. Cette partie contient en outre un chapitre spécialement dédié aux marchés publics, audits précontractuels compris.

Partie IV : $\quad$ Suggestions.

Publication

conjointe : Commentaires des entités contrôlées. 
Le Président de l'HCA soumet le rapport annuel au Président du Parlement. Ce rapport est débattu en séance plénière dans le cadre du contrôle parlementaire. II est ensuite publié dans la Gazette de l'État et sur le site Internet de la Cour, ce qui le rend accessible au public.

La déclaration annuelle de la Cour a lieu selon les méthodes de communication suivante : chaque année, avant la fin du mois de juin, le rapport annuel sur l'exécution du budget et le bilan de l'État, accompagnés de divers états financiers, est soumis par le Ministère des Finances au Bureau du Commissaire compétent de l'HCA qui, dans une période de deux mois et après avoir vérifié l'exactitude et la fiabilité des pièces, le renvoie avec son propre rapport au Bureau de la comptabilité générale de l'État. Dans les 20 jours suivant I'envoi, le Ministre des Finances soumet à la Cour ses vues et ses commentaires sur le rapport.

Après avoir pris en compte le rapport du Bureau du Commissaire compétent et les vues du ministre, l'HCA réunie en séance plénière statue sur la correction et la fiabilité du rapport annuel portant sur l'exécution du budget, le bilan et les autres états financiers transmis par l'État dans son rapport (la Déclaration), rapport soumis par le Ministère des Finances à la fin du mois d'octobre de l'année concernée.

Le rapport annuel sur l'exécution du budget, le bilan et les autres états financiers du gouvernement central, accompagnés de la Déclaration de I'HCA, sont déposés devant le Parlement et soumis à sa ratification à l'initiative du Ministre des Finances, au plus tard fin novembre chaque année et, dans tous les cas, avant la soumission du budget de l'État au Parlement pour l'année suivante.

\section{Procédures d'envoi des rapports au Parlement}

Récemment (2013 - 2014), les séances d'information et les présentations faites par l'HCA devant les commissions parlementaires (Commission du bilan et de la déclaration financière, Commission des affaires financières) ont eu lieu dans un contexte non formalisé.

\section{3) Procédures parlementaires utilisées dans le traitement des rapports de l'ISC}

La réglementation parlementaire en vigueur ne prévoit pas d'auditions devant les commissions parlementaires avec la participation de l'HCA.

Comme il l'a été évoqué plus haut, en 2014, deux rapports d'audit spéciaux (financier et conformité) ont été soumis pour délibération aux commissions parlementaires compétentes (à savoir la Commission de la déclaration financière, du bilan et de l'exécution du budget de l'État, la Commission permanente des affaires culturelles et de l'éducation et la Commission permanente des affaires sociales).

\section{Personnel}

Les informations concernant le personnel parlementaire assistant la ou les commissions qui examinent les rapports de l'ISC ne sont pas disponibles.

\section{Participation de I'ISC à l'examen de ses rapports par le Parlement}

Comme il l'a été mentionné ci-dessus, le Président de I'HCA soumet le rapport annuel au Président du Parlement. La participation de I'ISC aux débats concernés n'a pas encore été institutionnalisée. 


\section{4) Rapports de l'ISC}

Dans sa communication avec le Parlement, I'ISC se concentre sur des problèmes génériques / systémiques et sur la formulation de recommandations.

La configuration et le fonctionnement des systèmes de contrôle internes ne sont pas des sujets mis en avant dans les rapports de I'ISC.

Tous les rapports d'audit contiennent des conclusions et des opinions claires.

\section{5) Suivi des rapports de l'ISC}

Les conclusions et les recommandations qui ne sont pas mises en œuvre par les entités contrôlées sont reprises dans le rapport suivant.

Les audits de suivi ont été inclus dans le programme de contrôle annuel de 2016.

\section{6) Audits réalisés à la demande du Parlement}

L'HCA émet des opinions sur les projets de loi qui règlementent les éléments importants de la gestion financière des organes du gouvernement général, soumis par le Ministre des Finances, (Article premier, Loi $n^{\circ} 4129 / 2013$ ) ainsi que sur les projets de loi concernant les retraites inscrites dans les dépenses du budget de l'État, en vertu de l'Article 73 alinéa 2 de la Constitution.

\section{7) Programme de travail}

Étant donné la nature judiciaire des travaux de la Cour, le programme de contrôle annuel est décidé en séance plénière, de manière indépendante, sans consultation du Parlement ou suggestion de sa part.

\section{8) Niveau et fréquence des échanges}

Mis à part les éléments susmentionnés, aucun échange régulier n'a été institué.

\section{9) Perspective}

L'institutionnalisation peut améliorer la relation entre I'HCA et le Parlement si le statut judiciaire de la Cour est parfaitement respecté. 


\section{HONGRIE}

Bureau national d'audit de la Hongrie

\section{1) Cadre juridique}

Le Bureau national d'audit de la Hongrie (SAO) est l'institution supérieure d'audit financier et économique de l'Assemblée nationale, à laquelle elle rend compte. Avec ses constatations, ses recommandations et ses conseils liés à sa mission de contrôle, le SAO assiste l'Assemblée nationale, ses commissions et le travail des entités contrôlées, renforçant ainsi la bonne gouvernance des opérations publiques.

Le SAO exerce ses fonctions dans le cadre du plan de contrôle approuvé par son Président, qui communique ce plan et ses amendements à l'Assemblée nationale. Au sein du domaine de compétence qui lui a été attribué par la loi, le SAO mène des audits selon les décisions prises par l'Assemblée nationale ; il peut en outre réaliser des audits à la demande du gouvernement. La fréquence de ces audits est déterminée par la loi ou, en l'absence de dispositions juridiques applicables, par le Président du SAO.

Aux termes des dispositions juridiques, le SAO contrôle la gestion financière des partis politiques, l'utilisation des financements accordés par l'Assemblée nationale aux groupes de parlementaires représentant un parti politique, l'utilisation de subventions normatives et de subventions à des fins particulières et affectées aux églises et aux institutions et organismes entretenus et gérés par les églises, et les données liées à l'utilisation du budget concernant les coûts opérationnels spéciaux des services de sécurité nationaux.

\section{2) Modalités de soumission des rapports de l'ISC au parlement}

Le Président du SAO organise la soumission du rapport annuel du SAO et du rapport sur le budget central et son exécution (comptes finaux) à l'Assemblée nationale. Les rapports sont adressés au Président de l'Assemblée nationale.

L'Assemblée nationale discute du projet de loi relatif au budget central, ainsi que des opinions émises par le $\mathrm{SAO}$ et le Conseil budgétaire. Le gouvernement soumet le projet de loi sur les comptes finaux à I'Assemblée nationale au plus tard huit mois après la fin de l'exercice budgétaire. Ce projet de loi est transmis au SAO deux mois avant sa soumission à l'Assemblée nationale. Cette dernière discute du projet de loi sur les comptes finaux, ainsi que des opinions émises par le SAO.

Dans le rapport annuel préparé pour l'Assemblée nationale, le Président du SAO fournit des informations sur l'activité de contrôle et sur la gestion opérationnelle et financière de l'année antérieure, ainsi que sur les mesures prises suite aux constatations d'audit.

\section{Procédures d'envoi des rapports au Parlement}

Le rapport annuel du SAO est adopté par l'Assemblée nationale. Lors du le débat général conjoint portant sur le rapport d'activité et la gestion du SAO, le Président du SAO prononce le discours d'ouverture.

Les rapports sur le budget central et son exécution (comptes finaux) sont débattus le même jour que leurs projets de loi respectifs. À cette occasion, le Président du SAO est également chargé du discours d'ouverture de la séance. 


\section{Nombre et type de rapports}

Tous les rapports du SAO sont transmis par voie électronique aux parlementaires et aux personnes rattachées à l'Assemblée nationale.

En 2015, le SAO a officiellement soumis trois rapports à l'Assemblée nationale. Des notes d'information résumant les audits ont également été soumises sous la forme de lettres d'une ou deux pages.

Le plan de contrôle du SAO a été remis personnellement par son Président, mais ce plan n'est pas un rapport.

Comme les analyses ne sont pas des rapports, la loi n'oblige pas à les soumettre. Elles ont été envoyées uniquement à titre d'information, elles ne sont donc pas consignées dans les registres de l'Assemblée nationale et elles ne sont pas mises à l'ordre du jour des débats.

Si l'une des commissions décide de discuter d'un rapport du SAO, ce dernier est invité à donner des informations sur le rapport. La réunion plénière ne débat pas et n'enregistre pas ces rapports. Les commissions ont la liberté de décider des sujets qui figureront à l'ordre du jour.

\section{3) Procédures parlementaires utilisées dans le traitement des rapports de l'ISC}

Le rapport annuel du SAO est débattu par la commission parlementaire compétente qui fait une proposition de décision. Cette proposition fait également l'objet de délibérations au cours du débat général, en présence du Président du SAO qui répond aux questions. Une fois le rapport adopté, une décision parlementaire est prise. La commission peut faire appel à des experts externes également, mais il ne s'agit pas d'une pratique courante. Les débats sur les rapports de I'ISC peuvent se tenir en commission et / ou en séance plénière. Les Règles de procédure de l'Assemblée nationale accordent aux commissions la liberté de discuter de n'importe quels rapports. Elles sont normalement informées des lettres d'avertissement du SAO et de tout autre dispositif pertinent. Pour réaliser leurs travaux, les parlementaires s'appuient en outre sur les informations données par le SAO sur les thèmes en cours de discussion et contrôlés par le SAO. Tous ses rapports sont distribués par voie électronique aux membres de l'Assemblée nationale. Les parlementaires sont en droit d'adresser des questions au Président du SAO, qui devra répondre sous quinzaine, soit en personne soit par le biais de l'Assemblée nationale.

\section{Personnel}

Parmi les 17 commissions, une seule examine les trois rapports que le SAO a l'obligation de soumettre. Les minutes des débats sont prises par le greffier de l'Assemblée nationale.

Au sein du SAO, plusieurs membres du personnel participent, à différents niveaux, aux relations avec le Parlement. Le Président (en son absence, le Vice-président) du SAO et ses cadres responsables participent aux activités professionnelles des commissions. Les collègues du service juridique vérifient l'ordre du jour et préparent de la documentation écrite relative aux avant-projets de loi. Le Président du SAO a le droit d'intervenir à tout moment dans le débat. Un collègue est assigné à l'organisation de toutes les communications avec l'Assemblée nationale. Les collaborateurs du service de la communication et de la division internationale contribuent à la préparation des publications connexes, en hongrois et en langues étrangères. 


\section{4) Rapports de l'ISC}

Dans sa communication avec l'Assemblée nationale, le SAO ne se concentre pas sur des problèmes génériques / systémiques et sur la formulation de recommandations, mais le système de contrôle interne a constitué un sujet essentiel lors du contrôle des gouvernements locaux. Une étude sur ce thème a été publiée en janvier 2015.

\section{5) Suivi des rapports de I'ISC}

Le SAO transmet son rapport sur ses constatations d'audit au dirigeant de l'entité contrôlée qui met en place un plan d'action en réponse aux constatations. Au cas où ce dirigeant ne soumettrait pas de plan d'action dans les délais, ou soumettrait un plan d'action inacceptable, le Président du SAO pourrait engager une procédure pénale ou prononcer une sanction disciplinaire à l'encontre de l'organisme contrôlé. Le Président peut également demander la suspension des subventions ou d'autres indemnités versées à l'entité contrôlée par un sous-système des finances publiques et la possibilité de recevoir une partie des contributions de l'impôt sur le revenu à hauteur de $1 \%$. Le SAO peut vérifier, par un audit de suivi, la mise en œuvre des mesures prévues au plan d'action. Si ces mesures ne s'avèrent pas efficaces, ou s'il est impossible de les appliquer, ou si sa décision est justifiée par la nature ou la gravité de l'affaire concernée, le Président du SAO peut demander à la commission compétente de l'Assemblée nationale de discuter du rapport et des constatations, et d'entendre le directeur de l'entité contrôlée.

Le gouvernement n'est pas obligé d'établir un rapport sur la mise en œuvre des recommandations de I'ISC.

\section{6) Audits réalisés à la demande du Parlement}

Au sein de son domaine de compétence défini par la loi, le SAO peut mener des audits à la demande du gouvernement.

Dans la pratique, le Président du SAO soumet à l'Assemblée nationale les trois rapports élaborés en vertu des obligations juridiques et enregistrés par l'Assemblée nationale. Ces rapports sont le rapport annuel du SAO, I'opinion sur l'avant-projet de loi et le rapport de l'audit sur l'exécution du budget (comptes finaux). II y a trois soumissions par an. Le rapport annuel du SAO est déposé au printemps de l'année suivant l'année examinée. La soumission des rapports sur la proposition de budget et les comptes finaux dépend du moment où le gouvernement soumet les avant-projets de loi respectifs. II doit soumettre l'avant-projet de loi sur l'exécution du budget à l'Assemblée nationale au plus tard le 31 août. Les deux avant-projets de loi sont débattus avec l'opinion / le rapport du SAO qui les concerne.

\section{7) Programme de travail}

Le SAO exécute sa mission en s'appuyant sur le plan de contrôle (plan de travail annuel) approuvé par son Président. Ce dernier communique à l'Assemblée nationale le plan de contrôle et tout amendement. II n'y a pas de consultation préalable.

\section{8) Niveau et fréquence des échanges}

Il y a des échanges réguliers et quotidiens entre le Bureau de l'Assemblée nationale et le Secrétariat du Président et la Direction du SAO. Un collaborateur est spécifiquement nommé pour organiser tous les échanges avec l'Assemblée nationale. 
Comme le SAO est l'organe supérieur du contrôle financier et économique de l'Assemblée nationale, une grande importance est accordée à l'exploitation de notre travail. L'Assemblée nationale joue un rôle fondamental dans l'utilisation des rapports du SAO. Notre objectif principal est que les processus législatifs exploitent le plus possible nos constatations et nos recommandations. Aux yeux du SAO, il est extrêmement important que les législateurs puissent être informés au moment opportun et avec le niveau de détail nécessaire des incidences socio-économique à long terme et du contexte fiscal et monétaire d'un instrument législatif donné. Les constatations, les corrélations et les recommandations contenues dans les rapports aident les législateurs dans leurs prises de décision en s'appuyant sur les éléments d'information attestés par audits.

Pour garder le contact avec l'Assemblée nationale, le SAO a établi un accord de coopération avec le Bureau de cette dernière. Cet accord est soumis à un renouvellement permanent, en harmonie avec les changements organisationnels que peuvent connaître les deux institutions. Selon un principe de coopération mutuelle, le SAO donne et reçoit des informations directes et de grande portée. Nous recevons périodiquement de la documentation qui éclaire les réunions plénières, ainsi que des invitations aux réunions des commissions (dans certain cas avec des matériaux explicatifs).

Toutes les fois qu'un rapport du SAO est publié, il est automatiquement envoyé au Président de l'Assemblée nationale, ainsi qu'aux présidents et vice-présidents des commissions chargées des questions de contrôle et budgétaire. Les fonctionnaires de l'Assemblée nationale et les élus sont informés par courriel de la mise à disposition des rapports du SAO et des résumés rédigés à l'intention de la presse. Les rapports du SAO sont en outre directement téléchargeables sur le site Internet de l'Assemblée nationale. Les travaux de cette dernière sont suivis, évalués et utilisés quotidiennement par le SAO.

\section{9) Bonnes pratiques}

En qualité d'organe supérieur du contrôle financier et économique de l'Assemblée nationale, le SAO s'efforce de faire en sorte que ses audits et les constatations formulées dans ses rapports soient le plus largement utilisés dans les procédures législatives. En conséquence, il rédige régulièrement des recommandations à l'intention du programme législatif de l'Assemblée nationale, pour la session d'automne comme pour celle du printemps.

Dans les recommandations préparées et envoyées à l'Assemblée nationale pour le programme législatif du printemps 2016, le SAO a attiré l'attention sur les rapports déjà publiés en relation avec les avantprojets de loi à soumettre, ainsi que sur les audits inscrits dans son plan de contrôle qui seront exécutés durant la première moitié de l'année.

Pour atteindre ses objectifs stratégiques, le SAO a envoyé ses recommandations au Président, aux présidents adjoints et aux chefs de groupe de l'Assemblée nationale. Dans son courrier envoyé aux présidents de 11 commissions et de 8 sous-commissions, le SAO a spécifiquement souligné les thèmes qui pourraient présenter un intérêt pour les commissions. Dans le cadre de la coopération entre le SAO et l'Assemblée nationale, les recommandations sont en outre disponibles sur le site Internet de l'Assemblée nationale.

Dans le domaine de ses compétences, le SAO peut préparer des analyses et des études. En lien avec les activités de son Président, il élabore des analyses et des études qui aident le Conseil fiscal à étendre la performance de ses travaux. 
11) Y-a-t-il d'autres éléments concernant les relations actuelles entre I'ISC et le Parlement que vous souhaiteriez souligner, et quelles a été leur évolution au cours des cinq dernières années?

L'accent a été mis sur l'assistance que le SAO apporte à l'Assemblée nationale. II est extrêmement important pour le SAO que les législateurs puissent être informés des effets socio-économiques à long terme et du contexte financier et fiscal des divers instruments de législation au moment opportun, et de manière suffisamment détaillée. C'est pourquoi le nombre de notes d'informations, d'études et d'analyses a augmenté. 


\section{IRLANDE}

Bureau du Contrôleur général des comptes

\section{1) Cadre juridique}

Le Contrôleur général des comptes est un agent constitutionnel dont la fonction est définie par l'Article 33 de la Constitution irlandaise de 1937.

La législation qui encadre la mission du Contrôleur général des comptes et du bureau de I'ISC est essentiellement contenue dans la Loi (l'amendement) relative au Contrôleur général des comptes de 1993.

Les résultats des contrôles sont communiqués au Parlement principalement de deux manières :

Par des certificats ou des rapports d'audit qui fournissent une opinion d'audit relative à l'exactitude des états financiers / comptes.

Par des rapports sur la gestion financière d'organes individuels et sur d'autres questions susceptibles d'être un sujet de préoccupation pour le Parlement.

La Commission des comptes publics (PAC), qui est une commission permanente du Parlement, joue également un rôle-clé dans le processus de rendre des comptes au public. Le Contrôleur général des comptes (C\&AG) de l'ISC assiste aux réunions de la PAC en tant que témoin permanent. Les résultats des examens menés par le C\&AG de manière indépendante offrent un fondement solide aux enquêtes de la PAC. L'efficacité de la Commission est renforcée par le fait que les rapports du C\&AG constituent une source initiale d'informations et à son tour, la fonction de vérification du C\&AG gagne en influence et en efficacité parce que ses rapports sont examinés et suivis par la PAC.

La PAC examine et communique au Parlement son analyse des comptes contrôlés par le C\&AG, ainsi que ses rapports. Ces comptes sont les comptes des crédits des ministères et des bureaux gouvernementaux et les comptes des Comités de santé et des VEC, ainsi que les états financiers des organismes non commerciaux financés par l'État.

La PAC examine aussi d'autres rapports définis par la loi et rédigés par le C\&AG :

Le C\&AG rend rapport également sur son analyse des évaluations d'économie, d'efficience et d'efficacité (VFM).

Les autres rapports établis par le C\&AG au terme de la Loi (amendement) sur le Contrôleur général des comptes de 1993.

\section{2) Modalités de soumission des rapports de l'ISC au parlement}

L'ISC envoie les rapports d'audit financier et de performance à la Commission permanente des comptes publics du Parlement.

Les rapports de I'ISC traitent chacun d'un audit. L'ISC produit en outre des rapports transversaux sur des questions qui intéressent plusieurs ministères, ainsi que des rapports sectoriels, par exemple sur l'éducation. 
L'ISC publie un rapport d'activité annuel sur son site Internet, qui intègre les statistiques de performance de son activité d'audit.

Tous les ministères gouvernementaux contrôlés sont tenus par la loi de présenter leurs comptes au contrôle de l'ISC d'ici le 31 mars et l'ISC doit avoir terminé son audit au 30 septembre.

\section{Personnel}

L'ISC a un fonctionnaire qui travaille dans les bureaux du Parlement et qui fournit du matériel d'information à la PAC avant l'examen des comptes du gouvernement ou d'un organe de l'État et de tout rapport de performance lié.

\section{Nombre et type de rapports}

\begin{tabular}{|c|c|c|c|c|c|c|c|}
\hline & $\begin{array}{l}\text { Rapports } \\
\text { d'audit } \\
\text { financier }\end{array}$ & \begin{tabular}{|c|} 
Rapports d'audit \\
de conformité
\end{tabular} & $\begin{array}{l}\text { Rapports d'audit } \\
\text { de performance }\end{array}$ & $\begin{array}{l}\text { Autres rapports } \\
\text { ou documents }\end{array}$ & $\begin{array}{l}\text { Rapports } \\
\text { d'activité } \\
\text { annuels }\end{array}$ & \begin{tabular}{|c|} 
Rapports \\
demandés par \\
le Parlement
\end{tabular} & Remarques \\
\hline $\begin{array}{c}\text { Nombre de } \\
\text { rapports soumis }\end{array}$ & 42 & - & 32 & - & - & - & \\
\hline $\begin{array}{c}\text { Nombre de } \\
\text { rapports discutés } \\
\text { par le Parlement }\end{array}$ & & & & & & & \\
\hline $\begin{array}{l}\text { Soumis au } \\
\text { Président }\end{array}$ & 0 & - & 0 & - & - & - & \\
\hline $\begin{array}{l}\text { Soumis au } \\
\text { Président de la } \\
\text { commission } \\
\text { spécialisée }\end{array}$ & 0 & - & 0 & - & - & - & \\
\hline $\begin{array}{c}\text { Copies envoyées } \\
\text { aux autres } \\
\text { commissions } \\
\text { concernées }\end{array}$ & $\mathrm{N}$ & - & $\mathrm{N}$ & - & - & - & \\
\hline $\begin{array}{c}\text { Notes de synthèse } \\
\text { destinées à la } \\
\text { commission } \\
\text { parlementaire }\end{array}$ & 0 & - & 0 & - & - & - & \\
\hline $\begin{array}{c}\text { Résumés soumis au } \\
\text { Parlement }\end{array}$ & 0 & - & 0 & - & - & - & \\
\hline
\end{tabular}

3) Procédures parlementaires utilisées dans le traitement des rapports de l'ISC

La PAC est une commission parlementaire permanente. Elle joue un rôle-clé dans le processus de rendre des comptes au public. Le Contrôleur général des comptes (C\&AG) de l'ISC assiste aux réunions de la PAC en tant que témoin permanent. Les résultats de l'examen mené par le C\&AG de manière indépendante 
offre un fondement solide aux enquêtes de la PAC. L'efficacité de la Commission est renforcée par le fait que les rapports du C\&AG constituent une source initiale d'informations et à son tour, l'examen du C\&AG gagne en influence et en efficacité parce que ses rapports sont examinés et suivis par la PAC.

La PAC examine et communique au Parlement son analyse des comptes contrôlés par le C\&AG, ainsi que ses rapports. Ces comptes sont les comptes des crédits des ministères et des bureaux gouvernementaux et les comptes des Comités de sante et des VEC, ainsi que les états financiers des organismes non commerciaux financés par l'État.

\section{Personnel}

L'ISC nomme un fonctionnaire pendant le mandat de la Commission des comptes publics. Ce fonctionnaire sert de contact entre la Commission et l'ISC, et il donne aux membres de la Commission du matériel d'information et évoque des problématiques potentielles avant les réunions.

\section{Participation de I'ISC à l'examen de ses rapports par le Parlement}

Le C\&AG de I'ISC assiste aux réunions de la PAC en tant que témoin permanent pour répondre à toutes les questions posées par la PAC ou par des témoins sur les rapports en cours d'examen.

\section{4) Rapports de I'ISC}

Dans sa communication avec le Parlement, I'ISC se concentre sur des problèmes génériques / systémiques et sur la formulation de recommandations.

La configuration et le fonctionnement des systèmes de contrôle internes sont des sujets mis en avant dans les rapports de I'ISC.

Tous les rapports d'audit contiennent des conclusions et des opinions claires.

\section{5) Suivi des rapports de l'ISC}

L'ISC conserve en interne un enregistrement électronique de toutes les recommandations, accompagnées des délais de mise en œuvre, qui peuvent faire l'objet de rapports de suivi.

Après chaque audition, la PAC transmet les recommandations au ministère des Finances. La PAC produit un rapport portant sur les résultats de mise en œuvre des recommandations.

\section{6) Audits réalisés à la demande du Parlement}

Selon la Constitution et le cadre juridique, notamment selon la Loi (amendement) relative au contrôleur général des comptes, le C\&AG de I'ISC a un mandat étendu, des pouvoirs constitutionnels importants et des protections juridiques ; il n'est soumis au contrôle ou à la direction d'aucune autre autorité, et il a le pouvoir de rendre compte au Parlement sur toute question liée aux audits. II a un mandat particulier pour exécuter des audits financiers, de conformité et de performance ; il a le pouvoir de contrôle sur toutes les finances du gouvernement central, il a accès à la documentation et aux locaux des organismes contrôlés et il a le droit et l'obligation de rendre compte au Parlement s'il le juge utile.

L'ISC peut prendre en compte les conseils du Parlement, mais au final, elle a le pouvoir de choisir les audits et les rapports qu'elle va réaliser au titre de son mandat juridique. 
Le C\&AG de I'ISC a compétence exclusive pour décider des thèmes de performance qu'il examinera au titre de son mandat juridique. La PAC ou le Parlement ne peut pas l'obliger à exécuter un quelconque audit de performance, ni influencer son choix. Le Parlement / la PAC ne fait pas de suggestions quant aux thèmes d'audit. Si la PAC demande à I'ISC de mener un audit sur un sujet particulier en raison par exemple d'un traitement médiatique négatif, le C\&AG examine le sujet dans le contexte du mandat de I'ISC. Le programme de travail et l'importance du sujet seront pris en compte, mais la décision de donner suite relèvera exclusivement de I'ISC.

\section{7) Programme de travail}

Le Programme de travail annuel est décidé exclusivement par I'ISC. La PAC utilise les résultats du programme de travail annuel de I'ISC lorsqu'elle définit son agenda de travail pour l'année à venir. Voir également la réponse précédente.

\section{8) Niveau et fréquence des échanges}

L'ISC et la PAC se réunissent officiellement une fois par semaine pour étudier les rapports. Des sujets non planifiés à l'avance peuvent être évoqués avant les réunions.

\section{9) Bonnes pratiques}

L'affectation par I'ISC d'un agent de liaison à la PAC contribue à la bonne gestion des affaires administratives car les membres de la Commission sont parfaitement informés et ils peuvent poser des questions avant les réunions officielles avec témoins.

\section{0) Perspective}

Aucun point faible significatif n'a été relevé. 


\section{Kosovo*}

Bureau du Vérificateur général

\section{1) Cadre juridique}

Constitution : L'Article 138, Rapports du Vérificateur général (AG) du Kosovo, stipule :

1. Le Vérificateur général de la République du Kosovo s'adresse à l'Assemblée :

a) pour faire rapport sur l'exécution du budget de l'État ;

b)pour donner un avis sur le rapport du gouvernement concernant ses dépenses de l'année précédente avant qu'il ne soit adopté par l'Assemblée ;

c) pour informer l'Assemblé sur les conclusions des audits sur demande.

2. Le Vérificateur général de la République du Kosovo soumet à l'Assemblée un rapport annuel sur les activités du bureau.

Loi sur le Vérificateur général et le Bureau national d'audit de la République du Kosovo 05/ L-055, Articles 20 et 23 :

20.1.L'AG adopte un Plan de contrôle stratégique de trois ans, mis à jour tous les ans ;

20.2.L'AG adopte tous les ans un Plan de contrôle qu'il soumet à la Commission de surveillance des finances publiques, jusqu'au 31 octobre ;

20.3.L'AG peut, à la demande écrite de l'Assemblé ou du gouvernement, mener des audits supplémentaires.

23.2. Lorsqu'il fait rapport sur des audits de régularité juridique, le Bureau national d'audit soumet des rapports d'audit finaux à chaque institution contrôlée et à l'Assemblée au plus tard quatrevingt-dix (90) jours suivant la soumission de l'état financier annuel (AFS) final de l'Organisation du budget. Si l'institution contrôlée est une municipalité, le rapport d'audit final est en outre remis à l'Assemblée municipale concernée.

23.3. Dans le cadre de la vérification du rapport annuel soumis par le gouvernement sur l'exécution du budget du Kosovo, le Bureau national d'audit soumet le rapport d'audit annuel à l'Assemblée dans les délais définis par la Loi relative à la gestion et à la responsabilité des finances publiques.

23.7.L'AG présente tous les ans le rapport d'audit annuel à l'Assemblée réunie en séance plénière ;

23.8.L'AG peut à tout moment s'adresser à l'Assemblée pour discuter de toute question relevant de sa compétence. 


\section{2) Modalités de soumission des rapports de l'ISC au parlement}

Le NAO envoie séparément au Parlement les rapports d'audit de régularité et d'audit de performance, le rapport d'audit annuel sur le budget de l'État ainsi que son rapport de performance annuel. À la demande écrite de I'Assemblée ou du gouvernement, I'AG a la faculté de mener un audit de régularité ou de performance sur toute institution ou organisme, conformément à la loi.

Les rapports d'audit individuels sont adressés au Président et aux membres de la Commission de surveillance des finances publiques (COPF), tandis que le rapport d'audit annuel et le rapport de performance annuel sont présentés au Secrétaire général de l'Assemblée et au Président de la COPF, et une copie est envoyée au Bureau de liaison pour les institutions et agences indépendantes au sein de l'Assemblée.

Certains rapports sont assortis de délais.

- Selon la Loi sur la gestion et la responsabilité financière 03/L-048, I'AG prépare et soumet à l'Assemblée un rapport sur les états financiers des organismes budgétaires et des actions publiques de l'exercice budgétaire antérieur. Si l'organisme budgétaire est une municipalité, le rapport est en outre envoyé à l'assemblée municipale concernée. Ce rapport fournit l'opinion de I'AG, à savoir si les états financiers donnent une appréciation exacte et juste des finances de ces organismes budgétaires et ces actions publiques. Le rapport est soumis au plus tard le 31 juillet suivant la fin de l'exercice fiscal concerné par le rapport.

- L'AG remet à l'Assemblée le rapport d'audit annuel sur les états financiers annuels (AFS) du budget du Kosovo au plus tard le 31 août de l'année suivante.

- Chaque année, dans les trois mois précédant la fin de l'exercice comptable, l'AG dépose devant l'Assemblée le rapport annuel portant sur ses activités de contrôle. Ce rapport englobe le rapport de l'audit indépendant des comptes du NAO.

L'AG peut à tout moment exécuter un audit de performance auprès de n'importe qu'elle institution ou organisme, et son rapport final est soumis à l'Assemblée.

L'AG établit son plan de travail annuel avant de le soumettre dans les délais fixé par la loi. Il le présente à la COPF au début de la saison de contrôle.

Des copies des rapports d'audit de l'AG sont envoyées à l'organisme. Les rapports sont également rendus publics.

\section{Procédures d'envoi des rapports au Parlement}

Voir les réponses précédentes pour connaître les procédures d'envoi des rapports au Parlement.

L'équipe de vérification prépare à l'intention des membres de la Commission des notes informatives sur les rapports d'audit qui seront examinés en audition publique. Si nécessaire, les rapports et les notes informatives sont débattus par les parlementaires lors de la réunion préparatoire, qui se tient normalement la veille de l'audition publique. 
Nombre et type de rapports

\begin{tabular}{|c|c|c|c|c|c|c|c|}
\hline & $\begin{array}{l}\text { Rapports } \\
\text { d'audit } \\
\text { financier }\end{array}$ & $\begin{array}{c}\text { Rapports d'audit } \\
\text { de conformité }\end{array}$ & $\begin{array}{l}\text { Rapports d'audit } \\
\text { de performance }\end{array}$ & $\begin{array}{c}\text { Autres rapports } \\
\text { ou documents }\end{array}$ & $\begin{array}{c}\text { Rapports } \\
\text { d'activité } \\
\text { annuels }\end{array}$ & $\begin{array}{c}\text { Rapports } \\
\text { demandés } \\
\text { par le } \\
\text { Parlement }\end{array}$ & Remarques \\
\hline $\begin{array}{c}\text { Nombre de } \\
\text { rapports soumis }\end{array}$ & 100 & & 14 & & 1 & & Total 114 \\
\hline $\begin{array}{c}\text { Nombre de } \\
\text { rapports discutés } \\
\text { par le Parlement }\end{array}$ & 50 & & 4 & & 3 & & \begin{tabular}{|c|} 
Les rapports \\
d'audit annuels \\
pour 2013 et \\
2014 ont été \\
examinés en 2015 \\
en raison des \\
élections et de la \\
nouvelle \\
législation \\
instaurée en \\
2014.
\end{tabular} \\
\hline $\begin{array}{l}\text { Soumis au } \\
\text { Président }\end{array}$ & $\mathrm{N}$ & $\mathrm{N}$ & $\mathrm{N}$ & & $\mathrm{N}$ & & \begin{tabular}{|l} 
Les rapports du \\
NAO sont \\
adressés au \\
Parlement
\end{tabular} \\
\hline $\begin{array}{l}\text { Soumis au } \\
\text { Président de la } \\
\text { commission } \\
\text { spécialisée }\end{array}$ & 0 & 0 & 0 & & 0 & & \\
\hline $\begin{array}{c}\text { Copies envoyées } \\
\text { aux autres } \\
\text { commissions } \\
\text { concernées }\end{array}$ & $\mathrm{N}$ & $\mathrm{N}$ & $\mathrm{N}$ & & 0 & & \\
\hline $\begin{array}{l}\text { Notes de synthèse } \\
\text { destinées à la } \\
\text { commission } \\
\text { parlementaire }\end{array}$ & 0 & 0 & 0 & & 0 & & \\
\hline $\begin{array}{c}\text { Résumés soumis au } \\
\text { Parlement }\end{array}$ & & & & & & & \\
\hline
\end{tabular}

3) Procédures parlementaires utilisées dans le traitement des rapports de I'ISC

Les règles de procédure interne de la COPF régissent le traitement des rapports de l'AG déposés devant le Parlement. 
La COPF traite tous les rapports du NAO. Elle est présidée par un membre de l'opposition. La COPF traite en outre les rapports d'audit (autres que ceux de l'AG) comme le rapport annuel sur le fonctionnement du système de contrôle des finances publiques internes (PIFC) dévolu au secteur public.

La COPF ne nomme pas de rapporteur.

Le premier responsable (ministre, directeur général) de l'organisation est convié aux auditions publiques de la COPF afin de fournir un complément d'informations sur le rapport examiné.

Conformément aux orientations de l'Assemblée sur les affaires financières et les procédures, la COPF a la faculté de faire appel à des experts externes indépendants pour des sujets particuliers.

En commission ou séance plénière, la COPF examine le rapport de performance annuel remis par le NAO puis envoie un rapport contenant ses recommandations à la séance plénière dans les trois semaines suivant l'acceptation du rapport. La COPF examine les rapports d'audit du NAO. La priorité est décidée par vote. En l'absence de réglementation applicable, la COPF vote pour sélectionner les rapports qui seront examinés en séance plénière. Elle examine le rapport d'audit annuel du NAO puis envoie un rapport contenant ses recommandations à l'Assemblée au plus tard le $1^{\mathrm{er}}$ octobre de l'année ${ }^{37}$.

Dans certains cas, d'autres commissions peuvent examiner les rapports d'audit.

Aux auditions publiques, la COPF demande à l'organe contrôlé d'envoyer des rapports sur les actions qu'il a pris suite aux recommandations faites par l'AG. Les délais d'envoi de ces rapports sont généralement de trois mois. Ces rapports ne sont pas publiés.

\section{Personnel}

Le personnel assistant la COPF comprend deux personnes ayant une formation juridique et économique. Elles réalisent des études écrites, des analyses ou des notes d'informations pour préparer les débats sur les rapports de l'ISC ; elles élaborent les questions que la COPF posera aux auditions et elles rédigent des rapports préliminaires ou d'autres documents que doit produire la COPF.

\section{Participation de I'ISC à l'examen de ses rapports par le Parlement}

Le NAO prépare des notes d'informations qui, si nécessaire, seront discutées lors des réunions préparatoires avec les parlementaires.

Les représentants du NAO assistent toujours aux réunions parlementaires qui portent sur les rapports d'audit. Le niveau de représentation dépend de l'ordre du jour de la réunion et du rapport d'audit soumis à examen. Dans des cas particuliers, l'AG est invité. Une personne appartenant à la haute direction ou le directeur du département d'audit concerné par le rapport examiné participe à la réunion.

L'agent de liaison chargé des relations avec le Parlement suit toutes les auditions de la COPF, les séances plénières et, si nécessaire, les auditions des autres commissions. 


\section{4) Rapports de I'ISC}

Dans sa communication avec le Parlement, I'ISC se concentre sur des problèmes génériques / systémiques et sur la formulation de recommandations.

La configuration et le fonctionnement des systèmes de contrôle internes sont des sujets mis en avant dans les rapports de l'ISC.

Tous les rapports d'audit contiennent des conclusions et des opinions claires.

\section{5) Suivi des rapports de l'ISC}

L'ISC conserve une trace des conclusions et des recommandations acceptées par les entités contrôlées.

Le Parlement suit la mise en œuvre des recommandations que I'ISC formule dans ses rapports d'audit.

Le suivi de la mise en œuvre de nos recommandations de l'année précédente fait partie intégrante de nos travaux d'audit. Le nombre de recommandations non suivies reste préoccupant, et il est nécessaire de prendre des mesures efficaces pour améliorer la situation. À cet égard, la COPF demande des rapports sur les façons dont les recommandations de l'AG ont été mises en œuvre.

Alors que les différents organes contrôlés préparent des plans d'action et établissent des rapports sur la mise en œuvre des recommandations de l'AG, le gouvernement en général n'a pas de mesure de suivi concernant le rapport d'audit annuel. Les rapports de I'ISC ne sont pas utilisés lors du débat annuel sur les projets de budget.

\section{6) Audits réalisés à la demande du Parlement}

La Loi sur l'AG et le NAO 05/L-055 : Article 20.3. stipule :

3.7 L'AG peut, sur demande écrite de l'Assemblée ou du gouvernement, exécuter des audits supplémentaires.

En 2015, le Parlement a demandé à l'AG de mener un audit sur la radio-télévision du Kosovo. L'AG a toutefois le pouvoir discrétionnaire de ne pas donner suite.

\section{7) Programme de travail}

Le NAO établit son plan de travail annuel en matière de contrôle et le présente à la COPF. Alors que les audits de régularité sont obligatoires pour les organismes détenus à plus de $50 \%$ par l'État ou qui reçoivent des fonds provenant du budget du Kosovo ou qui produisent des dividendes pour ce budget, la discussion concerne les audits de performance, la COPF formulant éventuellement des suggestions. Toutefois, c'est l'AG qui décide du plan de travail final.

\section{8) Niveau et fréquence des échanges}

L'AG ou une personne appartenant à la haute direction et désignée par l'AG est présent à presque toutes les réunions de la COPF (au moins deux fois par mois). Si nécessaire, des échanges informels sont organisés avec le Président de la COPF. 
Si nécessaire, il y a des échanges au quotidien dans le cadre du travail.

Le fonctionnaire chargé de la communication est l'agent de liaison avec le Parlement. De manière générale, l'AG adjoint est chargé des relations avec le Parlement.

\section{9) Bonnes pratiques}

Sur recommandations de l'OAG (désormais le NAO), la COPF a organisé en 2015 plusieurs auditions pour examiner les rapports d'audit de performance portant sur l'entretien et la réparation des véhicules officiels ; dans le cadre de cette opération, elle a également examiné cinq rapports d'audit de régularité des institutions, qui se trouvaient dans le champ de contrôle de cet audit de performance. Le premier responsable de ces institutions a été invité aux auditions et la COPF lui a fait des recommandations dans le cadre de ces rapports.

\section{0) Perspective}

En collaboration avec la COPF, nous recherchons des moyens pour renforcer les mécanismes permettant d'augmenter le pourcentage de recommandations mises en œuvre faites par l'AG.

Pour rendre la communication plus efficace entre I'ISC et le Parlement, le NAO envisage la possibilité d'avoir un agent de liaison / vérificateur permanent au Parlement. La discussion n'en est qu'à ses débuts et les procédures sont en cours d'évaluation.

11) Y-a-t-il d'autres éléments concernant les relations actuelles entre l'ISC et le Parlement que vous souhaiteriez souligner, et quelles a été leur évolution au cours des cinq dernières années?

Nous considérons que les relations entre I'ISC et le Parlement sont durables et en constante évolution. Le Parlement appuie l'AG dans l'exercice de son mandat et le rôle joué par l'ISC est très apprécié. 


\section{LETTONIE}

Cour des comptes de Lettonie

\section{1) Cadre juridique}

La Cour des comptes de Lettonie (ISC de Lettonie) est tenue par la loi de soumettre le rapport et l'opinion des auditeurs sur le rapport annuel de la République de Lettonie portant sur l'exécution du budget de l'État et sur l'exécution des budgets des gouvernements locaux.

Chaque année, I'ISC de Lettonie est tenue de soumettre des opinions qualifiées au Président du Parlement portant sur l'exactitude et la fiabilité des états financiers annuels communiqués par les ministères ou les agences du gouvernement central.

L'ISC de Lettonie doit soumettre au Parlement et au cabinet des ministres ses rapports sur les audits financiers des organismes contrôlés lorsqu'elle a relevé des anomalies, ou lorsqu'aucune opinion n'a pu être formulée. Le Parlement doit en outre être informé de tous les audits de performance et de conformité exécutés par I'ISC, ainsi que de toute autre constatation pertinente.

\section{2) Modalités de soumission des rapports de l'ISC au parlement}

L'ISC est tenue par la loi de soumettre le rapport et l'opinion des auditeurs sur le rapport annuel de la République de Lettonie portant sur l'exécution du budget de l'État et l'exécution des budgets des gouvernements locaux (avant le 15 septembre). L'ISC soumet au Parlement les opinions avec réserves portant sur les ministères ou les agences du gouvernement central, ainsi que des rapports d'audit de performance et de conformité. L'ISC envoie le récapitulatif des actions réalisées suite aux recommandations sur trois ans maximum.

L'ISC établit des rapports sur chaque audit séparément. Son rapport sur le rapport annuel de la République de Lettonie est adressé aux responsables du ministère des Finances et au Parlement, tandis que d'autres rapports sont envoyés aux ministères correspondants, une copie étant adressée à la Commission des marchés et des contrôles (SPIRK) ainsi que, si nécessaire, aux autres commissions compétentes.

Après réception d'un rapport d'audit, la SPIRK fixe la date de réception de la réponse de l'entité contrôlée sur les conclusions de l'audit et sur les irrégularités détectées, ainsi qu'un plan d'action visant à éliminer ces anomalies. L'ISC soumet ensuite des remarques complémentaires sur les réponses qu'elle a reçues, puis la SPIRK tient des réunions avec les organismes concernés.

L'ISC est autorisée à recevoir des propositions de loi élaborées par le Parlement, à offrir son opinion si les promulgations de loi proposées risquent d'avoir une incidence sur les revenus fiscaux, les dépenses de l'État et sur les autres budgets des gouvernements locaux, ou bien d'engager des actions avec des biens publics, des ressources financières provenant de l'Union européenne ou d'autres organes internationaux et qui sont inscrites au budget national ou dans les budgets locaux.

\section{Procédures d'envoi des rapports au Parlement}

II n'y a pas de procédures spécifiques régissant l'envoi des rapports au Parlement. L'ISC présente ses travaux devant la SPIRK au Parlement. 
Nombre et type de rapports

\begin{tabular}{|c|c|c|c|c|c|c|c|}
\hline & $\begin{array}{l}\text { Rapports } \\
\text { d'audit } \\
\text { financier }\end{array}$ & $\begin{array}{l}\text { Rapports d'audit } \\
\text { de conformité* }\end{array}$ & $\begin{array}{l}\text { Rapports d'audit } \\
\text { de performance }\end{array}$ & \begin{tabular}{|c|} 
Autres rapports \\
ou documents
\end{tabular} & $\begin{array}{l}\text { Rapports } \\
\text { d'activité } \\
\text { annuels }\end{array}$ & $\begin{array}{c}\text { Rapports } \\
\text { demandés par } \\
\text { le Parlement }\end{array}$ & Remarques \\
\hline $\begin{array}{c}\text { Nombre de } \\
\text { rapports soumis }\end{array}$ & 27 & & 18 & 24 & 1 & - & \\
\hline $\begin{array}{c}\text { Nombre de } \\
\text { rapports discutés } \\
\text { par le Parlement }\end{array}$ & 27 & & 18 & 24 & 1 & - & \\
\hline $\begin{array}{l}\text { Soumis au } \\
\text { Président }\end{array}$ & 27 & - & 5 & - & - & - & \\
\hline $\begin{array}{c}\text { Soumis au } \\
\text { Président de la } \\
\text { commission } \\
\text { spécialisée }\end{array}$ & 27 & - & 18 & 24 & 1 & - & \\
\hline $\begin{array}{c}\text { Copies envoyées } \\
\text { aux autres } \\
\text { commissions } \\
\text { concernées }\end{array}$ & 1 & - & 4 & Sans objet & - & - & \\
\hline $\begin{array}{c}\text { Notes de synthèse } \\
\text { destinées à la } \\
\text { commission } \\
\text { parlementaire }\end{array}$ & & & & 47 (total) & & & \\
\hline $\begin{array}{c}\text { Résumés soumis au } \\
\text { Parlement }\end{array}$ & $2 * * *$ & - & 18 & 24 & 1 & - & \\
\hline
\end{tabular}

* Les audits de conformité sont intégrés dans les audits de conformité-performance

** La SPIRK du Parlement

*** Les récapitulatifs des audits financiers comprennent deux rapports résumés, un résumé du rapport annuel de la République de Lettonie et un résumé regroupant des rapports d'audit financiers sur l'exactitude et la fiabilité des états financiers annuels 2014 de 26 institutions d'État.

\section{3) Procédures parlementaires utilisées dans le traitement des rapports de l'ISC}

II n'y a actuellement aucune procédure particulière appliquée par le parlement pour examiner les rapports de l'ISC.

Il existe une commission dédiée au traitement des rapports de I'ISC, la Commission des dépenses publiques et du contrôle, qui est présidée par l'un de ses membres compétents. La procédure de sélection de ce membre est fondée sur des négociations politiques.

La commission ne nomme pas de rapporteur. 
Il existe des auditions auxquelles sont conviées les entités contrôlées et les autres personnes concernées afin d'apporter des clarifications sur les constatations de l'audit et sur l'avancement de la mise en œuvre des recommandations d'audit.

Lors de l'examen des rapports, la Commission peut faire intervenir des experts externes indépendants si elle le juge utile. Il est courant que des représentants de la société civile, tels que des organisations non gouvernementales, soient invités à faire part de leur opinion sur l'audit concerné. La Commission peut demander par écrit aux responsables publics un complément d'informations ou des clarifications.

Le débat sur les rapports de I'ISC se déroule uniquement au sein de la Commission des dépenses publiques et du contrôle.

Comme résultante d'un débat sur un rapport de I'ISC, le Parlement peut reconnaître une faute ou des imperfections (le cas échéant) et convoquer les représentants de l'entité contrôlée responsables pour leur demander une clarification approfondie des faits ainsi que l'application de certaines mesures.

L'ISC ne joue aucun rôle dans la procédure de décharge appliquée par le Parlement.

\section{Personnel}

Selon le règlement interne, le fonctionnement de la Commission parlementaire est assuré par deux consultants et un consultant principal. Ce personnel mène des études et des analyses approfondies, et il fait des présentations d'informations avant les auditions. Cette fonction d'assistance couvre en outre la surveillance de la mise en œuvre des recommandations d'audit, et leur suivi. Si les délais de mise en œuvre des recommandations ont été dépassés, ces assistants envoient au nom de la Commission une communication écrite aux représentants des entités contrôlées, ou les convient à des réunions sur place.

\section{Participation de l'ISC à l'examen de ses rapports par le Parlement}

L'ISC participe à la préparation des auditions de la Commission ou du Parlement. Avant les auditions, l'ISC se réunit souvent avec les membres de la Commission des dépenses publiques et du contrôle afin de discuter des constatations les plus récentes ou des rapports d'audit qui seront soumis prochainement. L'ISC peut être présente aux réunions et auditions, pour attirer l'attention sur les constatations et les problèmes évoqués dans ses rapports.

\section{4) Rapports de I'ISC}

I'ISC se concentre sur des problèmes génériques / systémiques, ainsi que sur l'identification d'incidents isolés. Si, pendant l'audit, les systèmes de contrôle internes révèlent des points faibles, ces derniers sont mis en évidence dans les rapports de I'ISC. Chaque rapport contient des conclusions et des opinions claires.

\section{5) Suivi des rapports de l'ISC}

L'ISC s'entretient régulièrement (tous les trimestres) avec l'entité contrôlée pour s'assurer que les recommandations d'audit sont véritablement mises en œuvre. Si les actions nécessaires ne sont pas prises, cet élément d'information est transmis au Parlement. Un système et un processus de travail ont été mis en place pour mettre en œuvre les recommandations d'audit. 
Le Parlement suit le processus de mise en œuvre par des communications écrites et par différents échanges avec les entités contrôlées responsables et les autres parties prenantes concernées.

L'ISC publie un rapport sur la mise en œuvre / l'ignorance des recommandations tous les six mois afin de suivre les derniers stades d'avancement du processus. Les recommandations d'audit sont prises en compte par le ministère des Finances pendant la préparation du budget national annuel.

\section{6) Audits réalisés à la demande du Parlement}

II n'y a pas de dispositions de cette nature. L'ISC est une institution entièrement indépendante, également lorsqu'elle planifie ses activités et met en œuvre son plan de contrôle. Le Parlement ne lui demande pas d'exécuter des audits particuliers.

\section{7) Programme de travail}

L'ISC ne consulte pas le Parlement sur son programme de contrôle annuel. Toutefois, chaque année, elle interroge le Parlement sur tout domaine de contrôle qui pourrait l'intéresser. L'ISC prend en compte et évalue ultérieurement les propositions qui lui ont été faites, lorsqu'elle élabore son plan de contrôle.

\section{8) Niveau et fréquence des échanges}

L'ISC coordonne le calendrier de soumission des rapports avec les commissions parlementaires. L'ISC et le Parlement se réunissent régulièrement. Des réunions supplémentaires peuvent être organisées suite à des constatations extraordinaires ou d'autres impératifs afin de consulter le Parlement. Actuellement, I'ISC ne dispose pas de personnel spécifiquement affecté aux relations parlementaires.

\section{9) Bonnes pratiques}

L'ISC a récemment interrogé les parlementaires sur le système budgétaire dans le but d'identifier toute problématique à laquelle serait confronté le Parlement lorsqu'il travaille sur des questions liées au budget.

\section{0) Perspective}

Souvent, les constatations d'audit fondamentales ne sont pas débattues par le Parlement réuni en séance plénière, et ce dernier n'émet pas d'avis global.

Un autre problème concerne le manque de communication des parlementaires de certains partis politiques qui sont membres de la Commission des dépenses publiques et du contrôle. Si l'information n'est pas relayée correctement aux factions correspondantes, les parlementaires non membres de la Commission risquent de ne pas avoir une vue d'ensemble de la situation et des constatations formulées par I'ISC.

11) Y-a-t-il d'autres éléments concernant les relations actuelles entre I'ISC et le Parlement que vous souhaiteriez souligner, et quelles a été leur évolution au cours des cinq dernières années?

Depuis cinq ans, la communication globale avec les commissions parlementaires a avancé. L'ISC peut s'adresser aux commissions compétentes si des fautes graves constatées dans les audits se situent dans leur champ de compétence. 


\section{LITUANIE}

Bureau national d'audit

\section{1) Cadre juridique}

\section{Constitution de la République de Lituanie}

Le Bureau national d'audit surveille la légalité de la possession et de l'utilisation des biens de l'État ainsi que l'exécution du budget.

L'Auditeur général soumet au Seimas (Parlement lituanien) une conclusion sur le rapport portant sur l'exécution du budget. (Article 134)

\section{Loi sur le Bureau national d'audit}

L'audit financier portant sur le Bureau national d'audit est exécuté par un organe d'audit désigné sur résolution du Seimas. (Article 8)

Le Bureau national d'audit soumet annuellement au Seimas :

a) Une opinion et un rapport d'audit sur l'ensemble des états consolidés de l'État ;

b) Une opinion et le rapport d'audit sur l'ensemble des états consolidés du Fonds d'assurancemaladie obligatoire ;

c) Une opinion et le rapport d'audit sur l'ensemble des états consolidés du Fonds d'assurance sociale ;

d) Une opinion et le rapport d'audit sur l'ensemble des états consolidés de la Trésorerie de l'État, dont l'estimation est approuvée par le Seimas ;

e) Une opinion et le rapport d'audit sur l'ensemble des états financiers, comprenant le rapport sur la dette publique ;

f) Le rapport annuel du Bureau national d'audit. (Article 9)

Afin d'exécuter ses fonctions avec efficience, le Bureau national d'audit doit définir le champ de ses contrôles. Le Bureau national d'audit établit chaque année le champ de ses contrôles dans des programmes d'audit public. Ces programmes sont confirmés par l'Auditeur général, après avoir évalué les recommandations données par la Commission de contrôle du Seimas.

Le Seimas peut, par résolution, demander au Bureau national d'audit d'exécuter un audit public dans sa sphère de compétence. (Article 14)

\section{2) Modalités de soumission des rapports de l'ISC au parlement}

\section{Statut du Seimas de la République de Lituanie (le Statut du Seimas)}

1. Le gouvernement prépare et, avant le 10 octobre, adopte et soumet au Seimas l'ensemble des états suivants : 
a) Un ensemble d'états consolidés de l'État comprenant le rapport sur l'exécution du budget et les états financiers consolidés ;

b) Un ensemble d'états consolidés du Fonds d'assurance sociale comprenant le rapport sur l'exécution du budget du Fonds d'assurance sociale et les états financiers consolidés du Fonds d'assurance sociale ;

c) Un ensemble d'états consolidés du Fonds d'assurance-maladie obligatoire comprenant le rapport sur l'exécution du budget du Fonds d'assurance-maladie obligatoire et les états financiers consolidés du Fonds d'assurance-maladie obligatoire ;

d) Des ensembles annuels d'états relatifs à d'autres fonds monétaires de l'État, dont les estimations sont approuvées par le Seimas, lorsque ces ensembles comprennent des rapports sur l'exécution du budget des états financiers ;

e) Un ensemble national d'états financiers.

2. L'Auditeur général présente, avant le 10 octobre, au Seimas une opinion et un rapport d'audit sur les ensembles d'états visés à l'alinéa premier du présent article.

3. Après réception de l'opinion de l'Auditeur général visé à l'alinéa 2 du présent article, et des résolutions préliminaires du Seimas présentées par le gouvernement concernant l'adoption des ensembles de déclarations visés à l'alinéa premier du présent article, le gouvernement établit un rapport sur les ensembles d'états visés à l'alinéa premier du présent article et les rapports supplémentaires de l'Auditeur général seront entendus lors des prochaines séances du Seimas.

4. Après avoir examiné les rapports visés à l'alinéa 3 du présent article lors des séances du Seimas, les commissions analysent les ensembles de rapports visés à l'alinéa premier du présent article et établissent leurs conclusions. (Article 225)

Les dirigeants des institutions d'État qui sont nommés par le Seimas ou bien dont la nomination est soumise à l'approbation du Seimas transmettent (habituellement avant le $1^{\mathrm{er}}$ mars) un rapport d'activité annuel sur l'institution. À réception dudit rapport, le Président du Seimas notifie le Seimas qui choisit la commission qui sera chargée d'examiner le rapport soumis. Après avoir examiné le rapport, la commission prépare une conclusion et une résolution préliminaire qui seront débattues en séance du Seimas, avec le rapport établi par le dirigeant de l'institution publique. Le Seimas adopte une résolution sur le rapport du dirigeant et sur les activités de l'institution. (Article 206)

\section{Loi relative au Bureau national d'audit}

Dans les cinq jours ouvrés qui suivent le traitement des documents d'audit public, l'Auditeur général soumet à la Commission de contrôle du Seimas des rapports d'audit de performance et des opinions et des rapports d'audit financiers (de régularité) lorsque le Bureau national d'audit publie une opinion avec réserves ou défavorable, ou bien le refus de certifier les comptes financiers. (Article 10)

L'ISC soumet en outre :

- Un rapport sur la mise en œuvre des recommandations résultant des audits des institutions publiques. 
- D'autres rapports établis en fonction des besoins et des sujets, qui sont liés à des audits individuels et qui présentent un intérêt pour le Seimas.

Les rapports d'audit sont normalement adressés au Président de la commission compétente (Commission de contrôle).

\section{Procédures d'envoi des rapports au Parlement}

Dans les cinq jours ouvrés qui suivent le traitement des documents d'audit public, l'Auditeur général soumet à la Commission de contrôle du Seimas des rapports d'audit de performance et des opinions et des rapports d'audit financier (de régularité) lorsque le Bureau national d'audit publie une opinion avec réserves ou défavorable, ou bien le refus de certifier les comptes financiers. (Article 10 de la Loi relative au Bureau national d'audit)

À l'exception des opinions obligatoires et du rapport annuel sur les activités de l'ISC qui sont directement soumis au Président du Seimas, l'ensemble des rapports d'audit sont déposés devant la Commission de contrôle. En règle générale, la Commission prépare les auditions pendant lesquelles les principales constatations d'audit sont présentées. Dans des cas individuels, un examen spécial des résultats d'audit peut être organisé lorsque l'entité contrôlée est invitée auprès de la Commission d'audit pour discuter avec I'ISC de la situation existante et des actions futures. En fonction des résultats de l'audit, la Commission peut adopter une décision qui contraint l'entité contrôlée à exécuter des actions visant à la mise en œuvre des recommandations formulées dans l'audit.

Un examen distinct des rapports d'audit est organisé au sein des commissions sectorielles auxquelles la Commission de contrôle transmet les rapports. Les opinions obligatoires soumises par I'ISC sont également examinées en séance plénière. 


\section{Nombre et type de rapport}

\begin{tabular}{|c|c|c|c|c|c|c|c|}
\hline & $\begin{array}{l}\text { Rapports } \\
\text { d'audit } \\
\text { financier }\end{array}$ & \begin{tabular}{|c|} 
Rapports d'audit \\
de conformité
\end{tabular} & $\begin{array}{l}\text { Rapports d'audit } \\
\text { de performance }\end{array}$ & $\begin{array}{c}\text { Autres rapports } \\
\text { ou documents }\end{array}$ & $\begin{array}{l}\text { Rapports } \\
\text { d'activité } \\
\text { annuels }\end{array}$ & $\begin{array}{c}\text { Rapports } \\
\text { demandés par } \\
\text { le Parlement }\end{array}$ & Remarques \\
\hline $\begin{array}{c}\text { Nombre de } \\
\text { rapports soumis }\end{array}$ & 32 & - & 21 & 3 & 1 & - & $\begin{array}{l}\text { L'ISC a soumis } \\
\text { également } 8 \\
\text { opinions } \\
\text { obligatoires }\end{array}$ \\
\hline $\begin{array}{c}\text { Nombre de } \\
\text { rapports discutés } \\
\text { par le Parlement }\end{array}$ & 13 & - & $27^{*}$ & 1 & 1 & - & 8 \\
\hline $\begin{array}{l}\text { Soumis au } \\
\text { Président }\end{array}$ & - & - & - & - & 1 & - & 8 \\
\hline $\begin{array}{l}\text { Soumis au } \\
\text { Président de la } \\
\text { commission } \\
\text { spécialisée }\end{array}$ & 23 & - & 20 & 3 & - & - & - \\
\hline $\begin{array}{c}\text { Copies envoyées } \\
\text { aux autres } \\
\text { commissions } \\
\text { concernées }\end{array}$ & 13 & - & 22 & - & - & - & - \\
\hline $\begin{array}{l}\text { Notes de synthèse } \\
\text { destinées à la } \\
\text { commission } \\
\text { parlementaire }\end{array}$ & 13 & - & 27 & 1 & - & - & - \\
\hline $\begin{array}{c}\text { Résumés soumis au } \\
\text { Parlement }\end{array}$ & - & - & - & - & - & - & - \\
\hline
\end{tabular}

* 14 audits exécutés en 2015, 13 audits dans les années antérieures.

** Lorsque les rapports sont débattus au Parlement, les représentants du Bureau national d'audit font des présentations en lien avec les rapports.

*** L'ISC soumet les rapports complets au Seimas et à ses commissions. Tous les rapports comprennent des résumés.

3) Procédures parlementaires utilisées dans le traitement des rapports de l'ISC

Au titre de son mandat, la Commission de contrôle doit examiner les rapports d'audit public et les opinions soumis par le Bureau national d'audit au Seimas et / ou aux commissions du Seimas. (Article 591 du Statut du Seimas)

Il existe une commission dédiée au traitement des rapports de I'ISC. Elle est présidée par un membre du ou des partis au gouvernement. Dans la législature précédente (2008-2012), elle était présidée par un membre de l'opposition. Cette décision est fondée sur des négociations politiques. 
La présentation des rapports d'audit public revient généralement à des représentants de l'ISC.

Il existe des auditions auxquelles est conviée l'entité contrôlée ou d'autres personnes concernées afin d'apporter des clarifications ou un complément d'informations.

Le débat sur les rapports de I'ISC se déroule au sein d'une (de) commission(s).

Des commissions autres que les commissions compétentes analysent également les rapports de l'ISC (si une autre commission prend cette décision à la demande de la commission spécialisée).

La décision d'une commission peut être le résultat d'un débat portant sur un rapport de l'ISC. La décision d'une commission oblige le gouvernement, un ministère ou une agence gouvernementale à mettre en œuvre les recommandations de I'ISC et à en informer la commission. La décision d'une commission est publiée sur le site Internet, et des communiqués de presse peuvent être publiés par le Parlement.

\section{Personnel}

II n'y a pas de personnel parlementaire spécialisé pour assister la ou les commissions affectées au traitement des rapports de I'ISC. Tout employé de la commission peut gérer les rapports de I'ISC. L'employé fait habituellement un résumé succinct du rapport de I'ISC et prépare quelques questions qui seront posées par la Commission. Comme il l'a été mentionné plus haut, une décision de commission ou un communiqué de presse parlementaire peut être publié.

\section{Participation de I'ISC à l'examen de ses rapports par le Parlement}

L'ISC procède à la présentation de ses rapports d'audit devant une commission parlementaire si cette dernière décide de les examiner. De manière générale, l'Auditeur général assiste aux réunions parlementaires de la Commission de contrôle lorsque les rapports de I'ISC sont débattus. Les rapports d'audit sont présentés par le directeur du service de I'ISC responsable de la problématique débattue ; le chef de l'équipe de contrôle du thème discuté assiste également aux réunions.

\section{4) Rapports de I'ISC}

Dans sa communication avec le Seimas, I'ISC se concentre sur des problèmes génériques / systémiques et sur la formulation de recommandations.

Ceci est particulièrement vrai lors de l'examen de rapports d'audit de performance qui portent sur des questions systémiques et stratégiques au niveau national et lors de la communication d'informations au Seimas. Deux fois par an, I'ISC élabore et soumet à la Commission de contrôle un rapport sur la mise en œuvre des recommandations d'audit. Ce rapport met en lumière les recommandations les plus importantes et ayant la plus forte incidence sur les domaines contrôlés de la gouvernance publique et dont la mise en œuvre a tardé.

En s'appuyant sur ces rapports, la Commission de contrôle adopte des décisions à l'intention du gouvernement, qui encouragent ce dernier à prendre des mesures pour mettre en œuvre les recommandations. La Commission soumet son rapport sur la mise en œuvre des recommandations aux commissions temporaires pour examen.

La configuration et le fonctionnement des systèmes de contrôle internes peuvent être des sujets mis en avant dans les rapports de I'ISC. La vérification des contrôles internes est particulièrement importante et 
pertinente dans les audits financiers (de régularité). Dans les audits de performance, les contrôles internes sont vérifiés autant que la situation l'exige afin de remplir l'objectif de l'audit. Dans le programme de contrôle annuel de 2016, I'ISC prévoit de mener un audit sur la mise en place d'un système de contrôle interne sur l'ensemble du secteur public, afin de révéler les problèmes systémiques et contribuer à les résoudre.

Tous les rapports d'audit contiennent des conclusions et des recommandations claires. Les conclusions des audits financiers (de régularité) offrent des opinions claires.

\section{5) Suivi des rapports de I'ISC}

Depuis 2008, I'ISC suit la mise en œuvre de chaque recommandation d'audit. Elle conserve une trace des conclusions et des recommandations acceptées par les entités contrôlées à l'aide d'un système d'informations interne qui enregistre chaque stade d'avancement jusqu'à la mise en œuvre finale. Le système a récemment reçu un nouvel outil qui permet à n'importe quel utilisateur externe à I'ISC de vérifier le stade de mise en œuvre des différentes recommandations.

La Commission de contrôle s'intéresse à la mise en œuvre des recommandations contenues dans les rapports d'audit de I'ISC en lui demandant de rendre compte deux fois par an sur le sujet. L'ISC établit un rapport spécifique sur les manquements en matière de mise en œuvre, y compris ceux relatifs à la configuration et au fonctionnement des systèmes de contrôle internes. Dans certains cas, cette démarche encourage le suivi parlementaire. Les ministères et les agences gouvernementales doivent soumettre des rapports de mise en œuvre des recommandations de I'ISC. L'opinion et l'audit de I'ISC traitent un ensemble d'états remis par l'État (comprenant le rapport de mise en œuvre du budget et des états financiers de l'État). Un ensemble national d'états financiers est utilisé lors des débats annuels sur les projets de budget.

\section{6) Audits réalisés à la demande du Parlement}

En votant une résolution, le Seimas peut demander au Bureau national d'audit d'exercer un audit public dans son domaine de compétence. (Article 14 de la Loi relative au Bureau national d'audit)

Dans la pratique, cette disposition est utilisée dans des cas exceptionnels, habituellement une fois par an maximum.

\section{7) Programme de travail}

Le Bureau national d'audit détermine tous les ans le champ de ses audits publics dans ses programmes d'audit public. Ces programmes sont confirmés par l'Auditeur général après analyse des recommandations de la Commission de contrôle du Seimas. (Article 14 de la Loi relative au Bureau national d'audit)

Dans la pratique, la Commission de contrôle du Seimas rassemble les suggestions de toutes les commissions parlementaires et les envoie à l'ISC. Cette dernière tient compte des informations reçues lorsqu'elle réalise l'analyse stratégique et évalue les risques relevés dans le secteur public par les contrôleurs qui ont préparé le programme de contrôle annuel.

\section{8) Niveau et fréquence des échanges}

Il y a régulièrement (presque une fois par semaine) des échanges entre le dirigeant de l'ISC et le président de la commission parlementaire compétente. 
La division juridique et la division du personnel (directeur de la division du personnel et conseiller de la division juridique) de I'ISC sont spécifiquement chargées des relations parlementaires au quotidien.

\section{9) Bonnes pratiques}

II y a des consultations bilatérales entre l'ISC et la Commission de contrôle du Seimas, y compris des consultations séparées avec son personnel.

L'ISC fournit des informations opérationnelles portant sur les audits à la demande des membres et du bureau de la Commission de contrôle.

L'ISC donne un avis sur les projets de loi qui concernent son activité, comme ceux relatifs à l'exécution du budget national, là a gestion des biens publics, aux marchés publics.

L'ISC organise des événements conjoints tels que la présentation du programme de contrôle annuel à l'intention de la Commission de contrôle et aux présidents des autres commissions et des factions parlementaires. Elle organise en outre des conférences sur les problématiques pertinentes telles que la discipline budgétaire.

\section{0) Perspective}

II arrive que les membres du Seimas ne s'intéressent pas aux rapports d'audit de l'ISC. Cette question est débattue en consultation bilatérale afin de trouver des moyens de rendre les présentations plus attrayantes. Cela reste toutefois un objectif à long terme.

11) Y-a-t-il d'autres éléments concernant les relations actuelles entre l'ISC et le Parlement que vous souhaiteriez souligner, et quelles a été leur évolution au cours des cinq dernières années?

L'ISC voit dans le Seimas et la Commission de contrôle un partenaire stratégique dans sa volonté de développer l'influence de ses recommandations d'audit ; en conséquence, elle fait très attention à maintenir des relations professionnelles, formelles et informelles. À chaque élection d'un nouveau Parlement et à chaque formation d'une nouvelle Commission de contrôle, l'ISC organise à leur intention des réunions pour leur expliquer l'importance du mandat de I'ISC, les particularités liées à l'exercice du contrôle public et l'impact de ses résultats qui entraînent des changements positifs dans le secteur public.

Les représentants de l'ISC participent aux auditions tenues par la Commission de contrôle, même si les rapports d'audits ne sont pas concrètement examinés. Des agents de liaison désignés travaillent en permanence avec le Bureau de la Commission. Les experts de l'ISC s'efforcent de participer à toutes les séances des commissions parlementaires et des commissions qui examinent des questions concrètes relatives aux résultats des contrôles ou aux problèmes systémiques touchant le secteur public.

La Division juridique et du personnel de l'ISC suit en permanence les informations liées aux projets de loi, et elle fournit aux contrôleurs les informations nécessaires. Si l'avant-projet de loi soumis à examen concerne la mise en œuvre des recommandations d'audit, I'ISC rédige des notes d'information individuelles sur les résultats d'audit qu'elle soumet à plusieurs reprises (par exemple lors de la préparation de la Loi sur les marchés publics) ce qui, de l'avis de I'ISC, peut être pertinent lors de l'examen de l'avant-projet de loi ou de ses amendements. Des conférences sont organisées conjointement avec la Commission de contrôle du Seimas et avec d'autres commissions parlementaires sur des problématiques importantes pour les deux parties afin d'attirer l'attention du Parlement et d'autres institutions sur les problèmes systémiques existant au niveau national. 


\section{LUXEMBOURG}

Cour des comptes

\section{1) Cadre juridique}

Le cadre juridique dans lequel s'inscrivent les obligations de I'ISC envers le parlement est défini par la Loi modifiée du 8 juin 1999 portant organisation de la Cour des comptes.

La Constitution et la loi sur le budget ne traitent pas des obligations de I'ISC envers le parlement.

En vertu de l'Article 4 de cette loi, l'ISC est tenue d'informer le Parlement si elle découvre que les entités contrôlées ont commis des actes frauduleux.

L'Article 5 de la Loi stipule que la Cour des comptes doit transmettre ses rapports spéciaux (accompagnés des réponses de l'entité contrôlée) au Parlement.

L'Article 6 stipule que le Parlement peut demander à l'ISC un avis sur la proposition de loi budgétaire. Le Parlement peut demander à I'ISC un avis sur les projets de loi ayant une incidence financière significative pour le Trésor public.

\section{2) Modalités de soumission des rapports de l'ISC au parlement}

L'ISC et le Grand-Duché du Luxembourg présentent régulièrement à la Chambre des députés des rapports spéciaux, qui rassemblent des audits financiers et de performance sur des domaines spécifiques.

Elle envoie en outre à la Chambre des députés des rapports annuels qui regroupent les audits financiers menés périodiquement auprès d'institutions publiques.

Pour terminer, elle présente annuellement à la Chambre des députés un avis sur la proposition de loi budgétaire, ainsi que sur les comptes généraux de l'État.

Elle soumet par ailleurs son rapport d'activité annuel au Parlement.

Comme il l'a été mentionné, les rapports spéciaux portent sur des audits individuels, tandis que les rapports annuels regroupent plusieurs audits financiers régulièrement réalisés.

Les rapports sont adressés au Président du Parlement.

La soumission des rapports au Parlement n'est pas assortie de délais.

\section{Procédures d'envoi des rapports au Parlement}

Conformément aux règles de procédure de l'ISC et du Parlement, tous les rapports doivent être soumis au Président du Parlement.

Les rapports sont présentés oralement devant la commission parlementaire compétente (par le conseiller / membre de la Cour compétent). Suite à cette présentation, le rapport est transmis à tous les parlementaires et rendu public sur le site Internet de I'ISC. Un communiqué de presse fait suite à la présentation. 
Nombre et type de rapports

\begin{tabular}{|c|c|c|c|c|c|c|c|}
\hline & $\begin{array}{l}\text { Rapports } \\
\text { d'audit } \\
\text { financier }\end{array}$ & \begin{tabular}{|l|} 
Rapports d'audit \\
de conformité
\end{tabular} & $\begin{array}{l}\text { Rapports d'audit } \\
\text { de performance }\end{array}$ & $\begin{array}{l}\text { Autres rapports } \\
\text { ou documents }\end{array}$ & $\begin{array}{l}\text { Rapports } \\
\text { d'activité } \\
\text { annuels }\end{array}$ & \begin{tabular}{|c|} 
Rapports \\
demandés \\
par le \\
Parlement
\end{tabular} & Remarques \\
\hline $\begin{array}{c}\text { Nombre de } \\
\text { rapports soumis }\end{array}$ & \begin{tabular}{|c|}
1 \\
(rapport \\
annuel qui \\
contient les \\
audits \\
financiers de \\
13 \\
organismes \\
publics).
\end{tabular} & $\begin{array}{c}1 \text { (identique aux } \\
\text { audits } \\
\text { financiers) }\end{array}$ & 1 & 2 & 1 & 0 & \\
\hline $\begin{array}{c}\text { Nombre de } \\
\text { rapports discutés } \\
\text { par le Parlement }\end{array}$ & 6 & & & & & & $\begin{array}{l}\text { Ce nombre inclut } \\
\text { les rapports } \\
\text { soumis avant } 2015 \text {. }\end{array}$ \\
\hline $\begin{array}{l}\text { Soumis au } \\
\text { Président }\end{array}$ & & & & & & & $\begin{array}{l}\text { Tous les rapports } \\
\text { sont soumis au } \\
\text { Président du } \\
\text { Parlement. }\end{array}$ \\
\hline $\begin{array}{l}\text { Soumis au } \\
\text { Président de la } \\
\text { commission } \\
\text { spécialisée }\end{array}$ & & & & & & & Voir ci-dessus. \\
\hline $\begin{array}{l}\text { Copies envoyées } \\
\text { aux autres } \\
\text { commissions } \\
\text { concernées }\end{array}$ & & & & & & & \\
\hline $\begin{array}{l}\text { Notes de synthèse } \\
\text { destinées à la } \\
\text { commission } \\
\text { parlementaire }\end{array}$ & & & & & & & \\
\hline $\begin{array}{c}\text { Résumés soumis au } \\
\text { Parlement }\end{array}$ & & & & & & & \\
\hline
\end{tabular}

3) Procédures parlementaires utilisées dans le traitement des rapports de l'ISC

Les procédures sont fixées par les Règles de procédure du Parlement, qui stipulent que les rapports de I'ISC doivent être adressés au Président du Parlement. 
II n'y a pas de commission officielle dédiée au traitement des rapports de I'ISC mais dans la pratique, ils sont traités par la Commission de contrôle sur l'exécution du budget. Cette commission est présidée par un membre du parti de l'opposition, selon la tradition.

La commission nomme un rapporteur pour traiter les rapports de I'ISC.

Des auditions ont lieu auxquelles sont conviés l'entité contrôlée, le ministre compétent ou d'autres personnes concernées, et des membres de I'ISC afin d'apporter des clarifications.

II n'y a pas de procédure établie régissant l'emploi d'experts externes indépendants et, de manière générale, la commission ne fait pas appel à des experts externes, sauf si elle décide de poursuivre l'enquête. Dans ce cas, elle peut ordonner un audit mené indépendamment par des experts externes.

Le débat sur les rapports de l'ISC se déroule en commission.

En règle générale, les rapports de l'ISC ne sont pas examinés par d'autres commissions.

\section{Personnel}

Un membre du personnel administratif est responsable des rapports de I'ISC. II a pour principale mission de rédiger des rapports sur les auditions de la commission. Toutefois, la commission peut lui demander de mener des études préparatoires aux débats.

\section{Participation de I'ISC à l'examen de ses rapports par le Parlement}

L'ISC ne participe pas à la préparation des auditions par la commission.

Le membre compétent de la Cour des comptes assiste aux réunions parlementaires de présentation des rapports, et il peut assister aux réunions qui débattent du rapport. II peut répondre aux questions et fournir des clarifications supplémentaires.

\section{4) Rapports de I'ISC}

Dans les rapports qu'elle soumet au Parlement, I'ISC se concentre entre autres sur les risques systémiques. Les systèmes de contrôle internes sont toujours mis en avant dans les rapports de l'ISC et ils constituent habituellement l'une de ses préoccupations majeures. Tous les rapports d'audit contiennent des conclusions et des opinions claires.

\section{5) Suivi des rapports de l'ISC}

L'ISC suit de manière informelle les conclusions et les recommandations acceptées par les entités contrôlées. Elle peut exécuter des audits de suivi.

Le Parlement débat de la mise en œuvre des recommandations de l'ISC et il en assure le suivi en audition.

\section{6) Audits réalisés à la demande du Parlement}

Aux termes de la Loi susmentionnée, le Parlement a la faculté de demander des audits à l'ISC. Dans la pratique, il demande des audits tous les deux ans environ. 
7) Programme de travail

Afin de préserver son indépendance, I'ISC ne consulte pas le Parlement sur son programme de travail.

\section{8) Niveau et fréquence des échanges}

Il y a régulièrement des échanges entre le Parlement et I'ISC. Le Parlement informe I'ISC de ses activités par courrier électronique, généralement une fois par semaine.

Dans la pratique, une personne est chargée des relations avec le Parlement. 


\section{MALTE}

Bureau national d'audit

1) Cadre juridique

La Constitution maltaise stipule que les comptes des ministères et des agences gouvernementales, ainsi que d'autres autorités ou organismes publics qui reçoivent des fonds publics doivent être contrôlés tous les ans par l'Auditeur général ( $A G)$ qui rend son rapport à la Chambre des représentants. La Loi relative à l'Auditeur général et au Bureau national d'audit (chapitre 39 des lois de Malte) stipule que le rapport d'audit annuel sur les comptes publics (qui comprend des audits de conformité et des audits financiers, y compris les audits concernant les états financiers de l'État) doit être soumis au Président de la Chambre des représentants au plus tard 12 mois après la fin de l'exercice budgétaire concerné par le rapport ; le Président dépose le rapport devant la Chambre des représentants à la séance suivant la réception du rapport.

La législation relative à l'audit public précise en outre que l'AG a la faculté de remettre des rapports spéciaux à la Chambre des représentants par l'intermédiaire du Président du Parlement, sur tout sujet très important ou urgent, ou qui portent sur des audits d'optimisation des ressources. Le Président du Parlement soumet le rapport devant la Chambre des représentants à la séance suivant sa réception.

La Loi sur le contrôle de l'État permet à l'AG de mener des enquêtes, soit personnellement soit par l'intermédiaire du Bureau national d'audit (NAO), et de rendre compte de tout ce qui touche aux finances de l'État, aux propriétés ou aux fonds gérés par les ministères, les divisions et les organismes d'État. L'AG peut en outre enquêter sur les opérations de sociétés ou d'autres organismes détenus au moins à $51 \%$ par l'État, ainsi que sur d'autres questions, dans les conditions prévues par la loi. Ces rapports spéciaux, présentés au Président de la Chambre des représentants à la séance suivante, sont préparés soit à l'initiative de l'AG, soit à la demande du ministre des finances. Au moins trois membres de la Commission des comptes publics (PAC) peuvent également demander un rapport spécial. La PAC est la commission permanente du Parlement chargée d'examiner les rapports du NAO et d'entendre les témoignages des entités contrôlées. Lorsqu'il examine les demandes formulées par le Parlement, l'AG n'est pas contraint de donner suite à ces demandes (bien que l'on s'attende à ce qu'il donne suite).

La Loi stipule par ailleurs que chaque rapport d'audit présenté doit mettre en avant tout élément que I'AG juge important et d'une nature qui devrait être portée à l'attention de la Chambre des représentants. Ce peut être l'absence de registres corrects, l'insuffisance de règles et de procédures destinées à préserver les biens publics, l'inefficacité des vérifications de l'évaluation, de la collecte et de l'attribution des revenus, une dépense non autorisée, des cas ou les deniers publics ont été dépensés de manière non économe, inefficiente ou inefficace, ainsi que l'absence de procédure satisfaisante servant à mesurer et rendre compte de l'efficacité des programmes de dépenses.

La Loi sur le contrôle de l'État demande également à l'AG de soumettre à la Chambre des représentants un rapport annuel sur le travail et les activités du NAO, en précisant, pour mener à bien cette mission, s'il a reçu toutes les informations et les explications nécessaires. Le rapport annuel sur le travail et les activités du NAO est soumis au Président de la Chambre des représentants au plus tard 12 mois après la fin de l'exercice budgétaire concerné par le rapport ; le Président soumet le rapport à la Chambre des représentants à la séance suivant la réception du rapport. 
Le cadre juridique n'exige pas que le NAO communique son plan de travail au Parlement ou à d'autres parties prenantes.

\section{2) Modalités de soumission des rapports de l'ISC au parlement}

Le NAO envoie au Parlement des rapports d'audit financier et de conformité, des rapports d'audit de performance, des rapports d'audit spéciaux et d'investigation, des rapports d'audit sur les systèmes informatiques, ainsi qu'un rapport annuel sur son travail et ses activités. Les résultats des audits financiers et de conformité sont réunis dans un seul rapport, intitulé le rapport annuel de l'AG. En ce qui concerne les audits de performance, les audits spéciaux et les investigations, ainsi que les audits sur les systèmes informatiques, le NAO établit des rapports individuels. Le rapport annuel sur le travail et les activités du NAO couvre le travail et les activités de l'année antérieure.

Tous les rapports sont adressés au Président de la Chambre des représentants qui les soumet à la Chambre à la séance suivante. Les rapports d'audit peuvent également être traités par la PAC.

Comme il l'a été précédemment mentionné, la législation en vigueur stipule que le rapport de l'AG et le rapport sur le travail et les activités du NAO doivent être établis tous les ans et soumis au Président de la Chambre des représentants au plus tard 12 mois après la fin de l'exercice budgétaire concerné par le rapport.

Les rapports d'audit de performance, les rapports d'audit spéciaux et d'investigation, et les rapports d'audit sur les systèmes informatiques sont soumis, une fois terminés, au Président de la Chambre des représentants.

\section{Procédures d'envoi des rapports au Parlement}

Chaque rapport établi par l'AG est présenté au Président de la Chambre des représentants. Pour faciliter la compréhension, tous les rapports d'audit comprennent une note de synthèse qui souligne les objectifs et les résultats marquants du rapport d'audit concerné. Tous les rapports sont accompagnés par un communiqué de presse qui reprend les principales problématiques et constatations évoquées dans le rapport.

Les rapports d'audit du NAO peuvent également être examinés par la PAC. Elle analyse les rapports établis par l'AG de manière détaillée et organise des auditions sur ces rapports. Des cadres-dirigeants des organismes contrôlés sont invités à participer à ces réunions de la PAC afin de fournir toute information ou explication que pourrait demander la Commission. La PAC s'assure ainsi que les constatations et les recommandations du NAO reçoivent l'attention qu'elles méritent.

L'AG joue un rôle actif pendant les séances de la Commission en répondant aux questions éventuellement posées par ses membres. Si nécessaire, d'autres collaborateurs qui ont participé à l'audit sont également présents. 
Nombre et type de rapports

\begin{tabular}{|c|c|c|c|c|c|c|c|}
\hline & $\begin{array}{l}\text { Rapports } \\
\text { d'audit } \\
\text { financier }\end{array}$ & \begin{tabular}{|c|} 
Rapports d'audit \\
de conformité
\end{tabular} & $\begin{array}{l}\text { Rapports d'audit } \\
\text { de performance }\end{array}$ & $\begin{array}{c}\text { Autres rapports } \\
\text { ou documents }\end{array}$ & $\begin{array}{c}\text { Rapports } \\
\text { d'activité } \\
\text { annuels }\end{array}$ & $\begin{array}{c}\text { Rapports } \\
\text { demandés } \\
\text { par le } \\
\text { Parlement }\end{array}$ & Remarques \\
\hline $\begin{array}{c}\text { Nombre de } \\
\text { rapports soumis }\end{array}$ & $1^{*}$ & $1^{*}$ & 3 & 4 & 1 & 5 & $\begin{array}{c}\text { Les rapports } \\
\text { demandés par le } \\
\text { Parlement sont des } \\
\text { rapports d'audit } \\
\text { spéciaux et } \\
\text { d'investigations. } \\
\text { Les « autres } \\
\text { rapports » sont } \\
\text { deux rapports } \\
\text { d'audit spéciaux et } \\
\text { d'investigations } \\
\text { ainsi que deux } \\
\text { rapports d'audit } \\
\text { informatiques. }{ }^{*} \text { Il y } \\
\text { a un rapport } \\
\text { d'audit annuel qui } \\
\text { comprend des } \\
\text { audits financiers et } \\
\text { des audits de } \\
\text { conformité. }\end{array}$ \\
\hline $\begin{array}{l}\text { Nombre de } \\
\text { rapports discutés } \\
\text { par le Parlement }\end{array}$ & 1 & 1 & Aucun & Aucun & Aucun & Aucun & $\begin{array}{c}\text { Les rapports } \\
\text { d'audit discutés } \\
\text { par la PAC en } 2015 \\
\text { étaient des } \\
\text { rapports publiés } \\
\text { les années } \\
\text { précédentes. La } \\
\text { Commission a } \\
\text { examiné par } \\
\text { ailleurs un contrat } \\
\text { signé par l'État. Le } \\
\text { rapport d'audit } \\
\text { financier et d'audit } \\
\text { de conformité } \\
\text { publié en } 2015 \text { est } \\
\text { examiné par la PAC } \\
\text { en } 2016 .\end{array}$ \\
\hline $\begin{array}{l}\text { Soumis au } \\
\text { Président }\end{array}$ & 0 & 0 & 0 & 0 & 0 & 0 & \\
\hline $\begin{array}{l}\text { Soumis au } \\
\text { Président de la } \\
\text { commission }\end{array}$ & 0 & 0 & 0 & 0 & 0 & 0 & \\
\hline
\end{tabular}




\begin{tabular}{|c|c|c|c|c|c|c|c|}
\hline spécialisée & & & & & & & \\
\hline $\begin{array}{c}\text { Copies envoyées } \\
\text { aux autres } \\
\text { commissions } \\
\text { concernées }\end{array}$ & $\mathrm{N}$ & $\mathrm{N}$ & $\mathrm{N}$ & $\mathrm{N}$ & $\mathrm{N}$ & $\mathrm{N}$ & \\
\hline $\begin{array}{c}\text { Notes de synthèse } \\
\text { destinées à la } \\
\text { commission } \\
\text { parlementaire }\end{array}$ & $\mathrm{N}$ & $\mathrm{N}$ & $\mathrm{N}$ & $\mathrm{N}$ & $\mathrm{N}$ & $\mathrm{N}$ & \\
\hline $\begin{array}{c}\text { Résumés soumis au } \\
\text { Parlement }\end{array}$ & $\mathrm{O}$ & $\mathrm{O}$ & $\mathrm{O}$ & $\mathrm{O}$ & $\mathrm{O}$ & $\mathrm{O}$ & \\
\hline
\end{tabular}

3) Procédures parlementaires utilisées dans le traitement des rapports de l'ISC

L'ordonnance $120 \mathrm{E}$ de la Chambre des représentants traite des pouvoirs et de la composition de la PAC. Elle a entre autre le pouvoir d'enquêter sur des questions liées aux comptes publics qui lui sont signalées par la Chambre des représentants, un ministre ou I'AG, ainsi que sur les dépenses publiques; elle est en outre habilitée à examiner les rapports et document connexes élaborés par l'AG. La PAC comprend sept membres, quatre qui appartiennent à la majorité au gouvernement et trois à l'opposition. Le Président de la Commission est un membre expérimenté de l'opposition ; il est nommé par le chef de l'opposition, après consultation avec le chef du parti majoritaire à la Chambre des représentants.

Les rapports du NAO ne sont pas débattus en séance plénière, mais pendant les réunions de la PAC. Cette dernière peut examiner en séance les rapports du NAO soumis à la Chambre des représentants. Elle est habilitée à demander des justificatifs aux hauts fonctionnaires des ministères et des divisions, ou travaillant pour d'autres entités publiques, comme les secrétaires permanents et les chefs de division. L'AG assiste aux séances de la PAC et joue un rôle actif dans la procédure.

Dans la pratique, les autres commissions parlementaires n'examinent pas les rapports du NAO, bien qu'elles aient la liberté de le faire.

Comme il l'a été mentionné plus haut, la Commission peut en outre, par le biais d'au moins trois membres, demander à I'AG de mener des investigations et autres enquêtes sur des questions relevant des attributions du NAO.

La PAC ne nomme pas de rapporteur et elle n'emploie pas d'experts. Toutefois, des représentants des médias peuvent assister aux séances de la PAC. Les transcriptions et les minutes des réunions de la PAC sont rédigées puis téléchargées sur son site Internet, tandis que les séances sont transmises en streaming sur le même site.

D'ordinaire, les débats publiés sont considérés comme étant les rapports officiels de la Commission. Toutefois depuis peu, un analyste de recherche aide l'employé de la PAC dans la rédaction des rapports. 
Le Parlement ne possède pas procédure de décharge établie mais la PAC a la faculté de demander au NAO de réaliser le suivi de ses rapports discutés en commission.

\section{Personnel}

Le seul personnel est l'employé de la PAC qui a pour fonction de coordonner les réunions, publier les ordres du jour, prendre les minutes, éditer et publier les transcriptions des débats, suivre toute les demandes faites par les membres de la PAC pendant les réunions, et actualiser la page de la PAC sur le site Internet du Parlement. Un extrait des activités de la Commission est publié dans le rapport annuel du Parlement.

Récemment, la Commission a pris la décision, lorsqu'elle n'examine pas le rapport du NAO et que des recommandations ont déjà été faites, de publier un rapport contenant ses propres recommandations.

\section{Participation de I'ISC à l'examen de ses rapports par le Parlement}

Le personnel du NAO, notamment les collaborateurs qui ont travaillé sur l'audit en cours d'examen, est prêt à fournir les informations contextuelles que la PAC juge nécessaires, le cas échéant.

L'AG, son adjoint et les autres membres gradés de son personnel, ainsi que les équipes de contrôle qui ont réalisé les différents audits, assistent aux réunions parlementaires où sont débattus les rapports d'audit. Ils jouent un rôle actif en répondant aux interrogations des membres de la PAC sur le rapport examiné.

\section{4) Rapports de I'ISC}

Dans sa communication avec le Parlement, le NAO se concentre essentiellement sur des problèmes génériques / systémiques et sur la formulation de recommandations. Toutefois, les erreurs importantes qui ne sont pas systémiques sont également communiquées au Parlement.

La configuration et le fonctionnement des systèmes de contrôle internes sont des sujets mis en avant dans les rapports de l'ISC.

Tous les rapports d'audit contiennent des conclusions et des opinions claires.

\section{5) Suivi des rapports de I'ISC}

Le suivi des rapports d'audit a pour principal objectif de fournir à la Chambre des représentants des informations en temps voulu sur les mesures mises en œuvre par l'entité contrôlée pour palier les manquements notés dans les rapports d'audit. Les travaux de suivi (notamment des audits financiers et de conformité) comprennent :

- L'examen systématique et en temps voulu de la gestion des recommandations d'audit faites par le NAO et des engagements que le directeur de la division a accepté d'honorer / de prendre pendant les auditions de la PAC;

- L'évaluation de l'action entreprise, pour voir si elle a remédié ou remédiera vraisemblablement aux problèmes identifiés pendant l'audit ;

- L'évaluation de la prise en compte des recommandations formulées dans le rapport initial ;

- Un rapport intégrant les résultats du suivi ; et 
- Déterminer si des actions supplémentaires devraient être prises dans le cadre d'un autre suivi ou audit.

Les rapports d'audit font au minimum l'objet d'un suivi :

- quand le problème est de nature récurrente ;

- quand un problème particulier porte sur une irrégularité très sérieuse ; ou

- quand la PAC demande la progression de la réalisation ou soumet des recommandations sur un problème.

Les problèmes peuvent faire l'objet d'un suivi jusqu'à ce que le NAO estime que les causes des manquements ont été corrigées de manière satisfaisante. La possibilité d'appliquer les recommandations originales est également prise en compte.

Le NAO conserve une trace des conclusions et des recommandations acceptées par l'entité contrôlée en mentionnant dans le rapport d'audit que l'entité accepte les conclusions et les recommandations du rapport.

Le Parlement s'intéresse à la mise en œuvre des recommandations formulées par I'ISC dans ses rapports d'audit en demandant aux entités contrôlées si elles ont mis en œuvre les recommandations. Si elles ne l'ont pas fait, les entités sont priées de proposer un calendrier de réalisation.

Lorsqu'elle applique un processus de suivi, I'ISC consacre un rapport spécifique à la mise en œuvre / l'ignorance des recommandations, y compris celles relatives à la configuration et au fonctionnement des systèmes de contrôle internes. Les travaux de suivi sont réalisés à l'initiative de I'ISC ou à la demande de la PAC. La PAC n'effectue pas elle-même de suivi.

Le gouvernement n'est pas obligé d'établir un rapport sur la mise en œuvre des recommandations de I'ISC. Les rapports de I'ISC ne sont pas régulièrement utilisés lors du débat annuel sur les projets de budget.

\section{6) Audits réalisés à la demande du Parlement}

La loi stipule que I'AG peut, à la demande d'au moins trois membres (sur sept) de la PAC, mener une enquête et rendre compte sur :

I.Toute question relative aux finances de l'État, aux propriétés ou au fonds gérés ou contrôlés par des ministères ou des bureaux gouvernementaux, ou des bureaux ou des organismes dont les comptes sont soumis à ses audits ;

II.Les comptes et les rapports financiers qui, en vertu de ou au titre d'une loi, sont déposés devant la Chambre des représentants ;

III.Les comptes des autorités publiques ou des organismes qui administrent, détiennent ou utilisent des fonds appartenant directement ou indirectement à l'État de Malte ;

IV.Les opérations des sociétés ou d'autres organisations détenues au moins à $51 \%$ par l'État de Malte ;

V.Toute autre affaire prévue par ou conformément à la loi. 
Dans la pratique, les membres de la PAC demandent des audits à la suite d'une violation présumée d'une politique ou d'une réglementation du gouvernement, ainsi que d'autres procédures établies ; une mauvaise gestion ou une faute présumée ; une utilisation inefficiente et inefficace présumée des ressources; ou un abus de pouvoir présumé. À titre d'exemple en 2015, cinq audits spéciaux et cinq rapports d'enquête ont été publiés suite aux demandes de la Commission de procéder à une enquête sur des ministères et des divisions du gouvernement central, ainsi que sur des organismes publics.

Le NAO a créé une section d'audit spécifiquement dédiée au traitement des audits spéciaux et des investigations. En raison de son indépendance, le NAO peut refuser d'exécuter une demande d'enquête de la PAC. Dans la pratique, cette section donne la priorité aux investigations demandées par la PAC. Une diligence raisonnable et le respect des exigences éthiques, y compris des dispositions de confidentialité, sont appliquées lors de l'exécution de tous les audits, entre autres des audits spéciaux et des investigations.

\section{7) Programme de travail}

L'ISC ne consulte pas le Parlement (commissions parlementaires, Président du Parlement et autres organes parlementaires) sur son programme de travail (pluri-)annuel en matière de contrôle.

\section{8) Niveau et fréquence des échanges}

Le NAO et la PAC se réunissent deux fois par an pour discuter du programme des séances de la PAC pour l'année concernée. D'autres réunions ad hoc relatives aux affaires de la PAC sont organisées selon le besoin.

Il n'y a pas d'échanges au quotidien dans le cadre du travail.

Les relations entre l'ISC et le Parlement sont gérées de façon ad hoc.

\section{9) Bonnes pratiques}

Les relations entre le NAO et la PAC sont collaboratives, sans porter atteinte d'une quelconque façon à l'indépendance du NAO.

Trois membres de la PAC peuvent demander au NAO d'exécuter des audits spéciaux ou des investigations. Depuis quelques années, ces demandes d'investigation sont plutôt fréquentes, et le NAO a enquêté sur des projets et des entités gouvernementaux majeurs. Les résultats de certains de ces audits spéciaux et de ces investigations ont donné lieu à des discussions approfondies au sein de la PAC.

Lorsqu'un parlementaire membre de la PAC a un conflit d'intérêt au regard d'un rapport d'audit discuté pendant une séance de la PAC, il est remplacé par un autre parlementaire.

Les pouvoirs et les fonctions de la PAC tels que définis par la législation et les ordonnances du Parlement ont visé à resserrer les liens entre le NAO et la PAC.

\section{0) Perspective}

La PAC n'examine pas et ne tient pas de séance sur tous les rapports du NAO.

II faut inciter la PAC à adopter une approche plus structurée / harmonisée lorsqu'elle examine les rapports du NAO. 
Par ailleurs, il semble que la PAC ne possède pas les capacités organisationnelles suffisantes, les ressources appropriées, la formation et l'accès aux conseils experts compétents pour exercer cette fonction de manière optimale.

11) Y-a-t-il d'autres éléments concernant les relations actuelles entre l'ISC et le Parlement que vous souhaiteriez souligner, et quelles a été leur évolution au cours des cinq dernières années?

Le NAO et la PAC entretiennent une relation collaborative. Plusieurs rapports d'audit du NAO sont examinés par la PAC.

Un audit spécial et une investigation majeure concernant les marchés publics du carburant ont été amplement discutés par la PAC. PAR SUITE, un certain nombre de rapports d'audit du NAO n'ont pas pu être débattus de manière efficace. Le NAO estime que le calendrier de la Commission, tout en consacrant plus de séances aux constatations sérieuses contenues dans les rapports du NAO, devrait garantir que tous les rapports du NAO soient discutés dans des délais raisonnables.

D'autres affaires, affectant les fonds publics, tels que des contrats / accords passés par l'État ou la politique monétaire de la Banque centrale, ont été débattues pendant les séances de la PAC.

Depuis que Malte est membre de l'Union européenne, le rapport d'audit annuel sur le budget de l'Union préparé par la Cour des comptes européenne, ainsi que les travaux du Membre maltais de la Cour des comptes européenne, sont discutés par la PAC.

Le NAO assiste à toutes les séances de la PAC, même à celles qui ne traitent pas de ses rapports. 


\section{MONTÉNÉGRO}

Institution nationale d'audit

\section{1) Cadre juridique}

Constitution du Monténégro, Article 144: L'Institution nationale d'audit soumet un rapport annuel au Parlement.

Loi relative à l'Institution nationale d'audit, Article 6 : Lors de l'adoption du budget du Monténégro et des comptes budgétaires finaux, I'Institution soumet au Parlement du Monténégro le récapitulatif des constatations faites sur le budget et l'état des actifs, soulignant les causes et les conséquences de toute erreur et irrégularité majeure et proposant des mesures pour les supprimer.

Loi relative à l'Institution nationale d'audit, Article 15: L'Institution rend compte au Parlement et au gouvernement, en règle générale après que l'entité contrôlée a exprimé son opinion sur les constatations de l'audit. L'Institution peut rendre compte au Parlement ou au gouvernement avant que l'entité contrôlée n'ait exprimé son opinion dans les cas où le rapport non remis dans les délais porterait préjudice, si les constatations étaient prématurément divulguées au public, ou si l'entité contrôlée n'avait pas exprimé son opinion dans les délais impartis.

Loi relative à l'Institution nationale d'audit, Article 18: L'Institution doit rendre compte au Parlement et au gouvernement : 1 ) en soumettant le rapport annuel ; 2 ) en soumettant des rapports spéciaux ; 3 ) en formulant des conseils fondés sur les constatations découlant de l'audit.

Loi relative à l'Institution nationale d'audit, Article 19: Le rapport annuel est soumis au Parlement et au gouvernement à la fin du mois d'octobre. Le Président du Monténégro, le Président du Parlement et le Premier ministre sont informés des circonstances de nature confidentielle, qui ont entraîné ou peuvent entraîner des préjudices financiers et autres préjudices étendus.

Loi relative à l'Institution nationale d'audit, Article 20: L'Institution informe le Parlement et le Gouvernement des questions particulièrement importantes au moyen de rapports spéciaux.

Loi relative à l'Institution nationale d'audit, Article 21: En s'appuyant sur les constatations de l'audit, I'Institution a la faculté de conseiller le Parlement et le gouvernement quant aux mesures financièrement significatives et aux projets importants. Au cas où elle aurait trouvé que les lois existantes engendraient ou pourraient engendrer des conséquences négatives, ou qu'elles ne produiraient pas les résultats escomptés, l'Institution peut faire des recommandations visant à les amender.

Loi relative à l'Institution nationale d'audit, Article 22 : L'Institution communique au Parlement et au gouvernement les raisons de retirer la réclamation pour dommages.

Loi relative à l'Institution nationale d'audit, Article 26: Pendant la procédure d'adoption des comptes budgétaires finaux du Monténégro, I'Institution adresse au Parlement un rapport sur l'audit des comptes budgétaires finaux. En s'appuyant sur les faits et les circonstances essentiels mis en lumière dans le rapport annuel de l'Institution, le Parlement sélectionne les mesures proposées et le calendrier de leur mise en œuvre. Le Parlement peut demander à I'Institution des clarifications additionnelles sur des faits et des circonstances spécifiques. 
Loi relative au budget et à la responsabilité budgétaire, Article 26 : I'Institution nationale d'audit vérifie l'application des critères de responsabilité fiscale dans le rapport annuel qu'elle soumet au Parlement. En s'appuyant sur cette vérification, le ministère des Finances propose au Gouvernement, et le Gouvernement propose au Parlement, un plan afin de respecter les limites numériques dépassées, conformément à la loi.

Loi relative au budget et à la responsabilité budgétaire, Article 67 : Le Gouvernement adopte la proposition de loi sur les comptes de fin d'exercice du budget de l'État d'ici la fin juin, puis il la soumet à I'Institution nationale d'audit, laquelle soumet un rapport sur l'audit des comptes de fin d'exercice du budget au Parlement avant le 15 octobre pour l'exercice budgétaire antérieur.

\section{2) Modalités de soumission des rapports de l'ISC au parlement}

L'Institution nationale d'audit (ISC) soumet au Parlement le rapport d'audit sur l'exécution du budget de l'État et le rapport d'audit annuel de l'ISC qui contient notamment : le rapport d'audit sur l'exécution du budget de l'État, le rapport sur la vérification des critères de responsabilité budgétaire, le rapport sur la mise en œuvre des recommandations issues du rapport d'audit sur l'exécution du budget de l'État de l'exercice budgétaire antérieur, les résumés des différents rapports d'audit finaux accompagnés des constatations essentielles et des recommandations, ainsi qu'un examen des autres activités de I'ISC (coopération avec le Parlement, coopération internationale, mise en œuvre du plan de développement stratégique, ressources humaines, formation, réalisation de projets, mise en œuvre des recommandations de l'Union européenne dans le cadre des négociations de l'adhésion du Monténégro, etc.).

L'ISC soumet aux commissions parlementaires les rapports d'audit financier, de régularité et de performance si sa section d'audit responsable estime ces rapports essentiels et si elle le mentionne dans les rapports.

L'ISC soumet ses rapports financiers tous les ans, lorsque son Conseil soumet le budget provisoire de I'Institution à la commission parlementaire chargée des finances.

\section{Procédures d'envoi des rapports au Parlement}

II n'y a pas de procédures écrites encadrant l'envoi des rapports de I'ISC au Parlement.

Le Président de I'ISC participe à la séance de la commission parlementaire de l'économie, des finances et du budget qui débat du rapport d'audit sur l'exécution du budget de l'État. Le Président de I'ISC, accompagné d'un membre du Conseil de I'ISC, participe également à la séance plénière pendant la présentation du rapport d'audit annuel de l'Institution.

En ce qui concerne les différents rapports d'audit, la section d'audit chargée d'un rapport donné peut participer à la séance des commissions parlementaires compétentes pendant le débat sur le rapport d'audit concerné. 
Nombre et type de rapports

\begin{tabular}{|c|c|c|c|c|c|c|c|}
\hline & $\begin{array}{l}\text { Rapports } \\
\text { d'audit } \\
\text { financier }\end{array}$ & $\begin{array}{c}\text { Rapports d'audit } \\
\text { de conformité }\end{array}$ & $\begin{array}{l}\text { Rapports d'audit } \\
\text { de performance }\end{array}$ & \begin{tabular}{|c|} 
Autres rapports \\
ou documents
\end{tabular} & $\begin{array}{c}\text { Rapports } \\
\text { d'activité } \\
\text { annuels }\end{array}$ & $\begin{array}{c}\text { Rapports } \\
\text { demandés par } \\
\text { le Parlement }\end{array}$ & Remarques \\
\hline $\begin{array}{c}\text { Nombre de } \\
\text { rapports soumis }\end{array}$ & 6 & $6^{38}$ & 3 & 1 & 1 & & \\
\hline $\begin{array}{c}\text { Nombre de } \\
\text { rapports discutés } \\
\text { par le Parlement }\end{array}$ & 6 & 6 & 3 & 1 & $1^{39}$ & & \\
\hline $\begin{array}{l}\text { Soumis au } \\
\text { Président }\end{array}$ & $\mathrm{N}^{40}$ & $\mathrm{~N}$ & $\mathrm{~N}$ & $\mathrm{O}^{41}$ & 0 & & \\
\hline $\begin{array}{l}\text { Soumis au } \\
\text { Président de la } \\
\text { commission } \\
\text { spécialisée }\end{array}$ & 0 & 0 & 0 & 0 & 0 & & \\
\hline $\begin{array}{c}\text { Copies envoyées } \\
\text { aux autres } \\
\text { commissions } \\
\text { concernées }\end{array}$ & $\mathrm{N}$ & $\mathrm{N}$ & 0 & $\mathrm{~N}$ & $\mathrm{~N}$ & & \\
\hline $\begin{array}{c}\text { Notes de synthèse } \\
\text { destinées à la } \\
\text { commission } \\
\text { parlementaire }\end{array}$ & $\mathrm{N}$ & $\mathrm{N}$ & $\mathrm{N}$ & $\mathrm{N}$ & $\mathrm{N}$ & & \\
\hline $\begin{array}{c}\text { Résumés soumis au } \\
\text { Parlement }\end{array}$ & $\mathrm{N}$ & $\mathrm{N}$ & $\mathrm{N}$ & $\mathrm{N}$ & $\mathrm{N}$ & & \\
\hline
\end{tabular}

3) Procédures parlementaires utilisées dans le traitement des rapports de l'ISC

Les Règles de procédure du Parlement ne définissent pas de procédure dédiée au traitement des rapports de I'ISC. Les commissions parlementaires se servent des informations contenues dans les rapports de l'ISC pour organiser des consultations, des auditions et autres discussions thématiques.

L'audit de conformité fait partie de l'audit financier.

Le rapport d'audit annuel comprend les résumés de tous les audits exécutés d'octobre de l'année précédente à octobre de l'année en cours.

Sur ces six audits financiers (de conformité), l'ISC a soumis au Président du Parlement un rapport d'audit sur l'exécution du budget de l'État.

Cette donnée fait référence au rapport sur l'évaluation de la mise en oeuvre des critères de responsabilité fiscale, qui est fait partie intégrante du rapport d'audit annuel. 
Les Règles de procédure du Parlement ne prescrivent pas de commission parlementaire spéciale dont la seule obligation serait de traiter les rapports d'audit de I'ISC. La Commission de l'économie, des finances et du budget est principalement chargée de cette tâche.

La Commission de l'économie, des finances et du budget est présidée par un parlementaire appartenant à la minorité au Parlement. II s'agit d'une pratique établie.

Les Règles de procédure du Parlement prévoient que la Commission puisse faire appel à des experts professionnels indépendants. Elle peut demander aux personnes qui assistent à la séance des informations complémentaires sur un thème spécifique. Cette pratique est normalement utilisée lorsque la Commission organise des auditions. Le personnel qui assiste la Commission analyse le matériau et l'information soumis, puis le remet aux membres de la Commission pour information.

L'entité contrôlée ou d'autres personnes concernées sont invitées à l'audition afin d'apporter des clarifications ou un complément d'informations.

Le débat sur les rapports de l'ISC se déroule au sein d'une (de) commission(s) et en séance plénière.

Mise à part la Commission de l'économie, des finances et du budget, d'autres commissions analysent les différents rapports de I'ISC s'ils portent sur des problématiques dans leur domaine de compétence (par exemple le rapport d'audit sur le ministère des Finances a été discuté par la Commission parlementaire sur la sécurité et la défense).

Le Parlement produit un rapport (minutes) ou une conclusion sur son débat. Ces rapports (minutes) et conclusions sont publiés sur le site Internet du Parlement. Ce dernier adopte les conclusions qui contiennent les recommandations essentielles du rapport d'audit sur l'exécution du budget de l'État ; les conclusions sont publiées dans le Journal officiel du Monténégro. Ces conclusions sont proposées par la Commission de l'économie, des finances et du budget au Parlement, qui les adopte sous la forme de ses propres conclusions.

\section{Personnel}

Les membres de la Commission de l'économie, des finances et du budget sont assistés par le Secrétariat de la Commission. Actuellement, le Secrétariat compte cinq conseillers de haut rang.

Le Secrétariat de la Commission est chargé de rédiger des notes de synthèse, des analyses et des conclusions préliminaires à l'examen des rapports de I'ISC par la Commission. Outre l'analyse des avantprojets de loi, il est responsable d'analyser l'audit de I'ISC sur l'exécution du budget de l'État et, plus succinctement, le rapport annuel portant sur les audits réalisés ; il est également chargé de récapituler la mise en œuvre des conclusions parlementaires liées aux recommandations faites dans l'audit précédent concernant l'exécution du budget de l'État.

\section{Participation de I'ISC à l'examen de ses rapports par le Parlement}

Le Président, accompagné du membre compétent de son Conseil ou par le directeur de la division du contrôle, assiste aux réunions parlementaires où sont débattus les rapports de I'ISC. II présente les constatations essentielles ainsi que les recommandations des rapports transmis. L'ISC peut fournir un complément d'informations aux commissions parlementaires avant et après les auditions. 


\section{4) Rapports de I'ISC}

Les rapports d'audit financiers et de régularité abordent le fonctionnement des systèmes de contrôle internes des organismes contrôlés, et ce domaine est clairement mis en avant dans les rapports de l'ISC.

Puisque d'ordinaire, I'ISC regroupe des audits financiers et de régularité dans un seul rapport, elle donne deux opinions, l'une sur les états financiers et l'autre sur la conformité envers les critères, les lois et les arrêtés applicables.

Si l'audit de régularité constitue un audit indépendant, I'ISC donne également une opinion claire.

Tous les rapports d'audit contiennent des conclusions claires.

\section{5) Suivi des rapports de l'ISC}

L'ISC conserve les conclusions et les recommandations dans un fichier Word / Excel à l'intention des entités contrôlées.

En ce qui concerne les recommandations faites dans le rapport d'audit sur l'exécution du budget de l'État, I'ISC soumet au Parlement un rapport sur la mise en œuvre des recommandations faites durant l'exercice budgétaire antérieur. II existe au sein de I'ISC un mécanisme de suivi des actions réalisées, et il fonctionne bien. Comme ces recommandations sont transmises sous la forme de conclusions parlementaires (publiées dans le Journal officiel du Monténégro), la Commission de l'économie, des finances et du budget suit sans difficulté la mise en œuvre des recommandations.

Le Parlement exige du gouvernement d'élaborer un plan d'action prenant en compte les recommandations de I'ISC et de rendre compte des progrès accomplis devant le Parlement. Ce programme comprend la création d'une équipe de coordination des ministres et des directeurs des organes de l'État, qui sera chargé de surveiller l'application du plan d'action. Les entités contrôlées concernées par le rapport sur l'exécution du budget de l'État établissent des rapports sur la mise en œuvre des recommandations, et le gouvernement dépose pour sa part des rapports trimestriels devant le Parlement.

Si la Commission de l'économie, des finances et du budget tient une audition, elle adopte généralement sa conclusion, sur laquelle l'entité contrôlée fonde le rapport de mise en œuvre des recommandations.

Ce mécanisme de suivi fonctionne également bien pour les rapports d'audit sur les états financiers annuels consolidés des partis politiques, qui sont exécutés chaque exercice budgétaire.

En ce qui concerne les rapports d'audit individuels, le Loi relative à l'ISC stipule que les entités contrôlées sont tenues de soumettre leur rapport sur la mise en œuvre des recommandations dans les délais définis par I'ISC. Les entités contrôlées soumettent leurs rapports de façon régulière. Le directeur de la section d'audit responsable a la faculté de demander à l'auditeur interne de l'État de vérifier l'exactitude des informations fournies dans le rapport et de rédiger des minutes d'audit indépendantes. Si l'entité contrôlée n'a pas réagi aux recommandations faites dans le rapport d'audit, le directeur de la section d'audit compétente $: 1$ ) peut octroyer un nouveau délai pour les recommandations partiellement mises en œuvre, ou pour les recommandations de moindre importance ; 2 ) peut proposer un audit de suivi inclus dans l'audit annuel réalisé par I'ISC si le nombre de recommandations ignorées ou partiellement mises en œuvre est important, ou si les recommandations revêtent une grande importance. 
Les rapports de I'ISC sont généralement utilisés lors des débats en séance plénière, notamment ceux adressés aux entités contrôlées qui ont reçu une opinion défavorable ou avec réserves.

\section{6) Audits réalisés à la demande du Parlement}

La Loi relative à I'ISC stipule que I'ISC choisit les entités à contrôler de manière autonome, à savoir le thème, le périmètre, le type, le calendrier et la méthode de contrôle, sauf disposition contraire prévue par cette Loi. L'ISC est tenue de vérifier les comptes budgétaires finaux une fois par an. La Loi sur le budget et la responsabilité budgétaire impose à I'ISC I'obligation d'évaluer l'application des critères de responsabilité budgétaire. La Loi sur le financement des partis politiques impose à I'ISC I'obligation de contrôler les états financiers annuels des partis politiques dont les recettes dépassent EUR 10000.

II n'y a pas de disposition juridique permettant au Parlement de demander des audits.

La Commission de l'économie, des finances et du budget exprime son opinion pendant le débat consacré aux différents besoins en matière d'audit. Le Sénat de I'ISC tient compte de ces opinions et dans certains cas, l'ISC les inscrit dans son plan de contrôle annuel.

\section{7) Programme de travail}

Le Sénat adopte le plan de contrôle annuel de l'année suivante avant la fin de l'année en cours. La procédure encadrant la préparation du plan de contrôle annuel est clairement définie dans l'instruction portant sur la méthodologie d'exécution des audits financiers et de régularité.

La procédure qui encadre la proposition et la sélection des audits à inscrire dans le plan de contrôle annuel est définie par plusieurs critères. Parmi ces critères, il y a le fait de savoir si l'entité a été contrôlée durant la période précédente, le volume des ressources publiques utilisées par l'entité, les résultats des contrôles précédents et le niveau de mise en œuvre des recommandations d'audit. Les informations sur les mesures prises par l'entité à contrôler communiquées par le Parlement, le gouvernement et les autres autorités nationales, les gouvernements autonomes locaux et les autres organismes indiquent s'il est nécessaire de contrôler l'entité en question ; à ces fins, d'autres sources d'informations sont également prises en compte, comme par exemple des informations publiques, la société civile et les courriers des citoyens. L'importance de la thématique de contrôle vue de l'intérêt public est également prise en compte.

Le plan de contrôle annuel est une donnée confidentielle interne à l'ISC, mais cette dernière notifie aux organismes le début du contrôle.

\section{8) Niveau et fréquence des échanges}

Le Conseil de I'ISC entretient des échanges réguliers dans le cadre du travail avec le Président de la Commission de l'économie, des finances et du budget. Une unité de I'ISC est chargée de la coopération internationale et des relations avec le Parlement, le gouvernement et les médias. II y a régulièrement des échanges entre le personnel de I'ISC et celui assistant la Commission de l'économie, des finances et du budget.

La fréquence de ces contacts dépend des besoins de l'ISC et du Parlement. 


\section{9) Bonnes pratiques}

Le Parlement adopte les recommandations faites lors de l'audit sur l'exécution du budget de l'État en rédigeant ses propres conclusions, qui sont publiées dans le Journal officiel du Monténégro.

\section{0) Perspective}

II manque des procédures écrites destinées au traitement des rapports d'audit.

Il manque un mécanisme de suivi destiné à la mise en œuvre des recommandations faites dans les rapports d'audit individuels. 


\section{POLOGNE}

Bureau supérieur d'audit

\section{1) Cadre juridique}

Les obligations de l'ISC envers le Parlement (par exemple la communication du plan de travail de I'ISC, l'obligation de rendre compte et la prise en compte des audits demandés par le Parlement) sont définies dans la Constitution de la République de Pologne et par la Loi du 23 décembre 1994 relative au Bureau supérieur d'audit.

\section{2) Modalités de soumission des rapports de l'ISC au parlement}

L'ISC (NIK) soumet au Parlement les rapports suivants : rapports d'audit financier (6 en 2015), rapports d'audit de conformité (39 en 2015), rapports d'audit de performance (87 en 2015), rapports sur l'exécution du budget de l'État de divers postes budgétaires (92 en 2015), analyse de l'exécution du budget de l'État de l'année antérieure (1 en 2015) et rapport d'activité annuel (1 en 2015).

\section{Procédures d'envoi des rapports au Parlement}

Le rapport d'activité annuel du NIK et le plan de travail annuel sont soumis, après discussion, à l'approbation de la Commission de contrôle de l'État. Les résultats des audits sont soumis au Parlement. 
Nombre et type de rapports

\begin{tabular}{|c|c|c|c|c|c|c|c|}
\hline & $\begin{array}{l}\text { Rapports } \\
\text { d'audit } \\
\text { financier }\end{array}$ & $\begin{array}{l}\text { Rapports } \\
\text { d'audit de } \\
\text { conformité }\end{array}$ & $\begin{array}{l}\text { Rapports d'audit } \\
\text { de performance }\end{array}$ & $\begin{array}{c}\text { Autres } \\
\text { rapports ou } \\
\text { documents }\end{array}$ & $\begin{array}{c}\text { Rapports } \\
\text { d'activité } \\
\text { annuels }\end{array}$ & \begin{tabular}{|c|} 
Rapports \\
demandés par \\
le Parlement
\end{tabular} & Remarques \\
\hline $\begin{array}{c}\text { Nombre de } \\
\text { rapports soumis }\end{array}$ & 6 & 39 & 87 & \begin{tabular}{|c|}
92 \\
(exécution du \\
budget de \\
I'État de divers \\
postes \\
budgétaires) \\
1 \\
(analyse de \\
I'exécution du \\
budget de \\
l'État dans \\
l'année)
\end{tabular} & 1 & Sans objet & \\
\hline $\begin{array}{c}\text { Nombre de } \\
\text { rapports discutés } \\
\text { par le Parlement }\end{array}$ & & & 59 & 86 & 1 & & \begin{tabular}{|c|} 
Au total, le \\
Parlement et ses \\
commissions ont \\
discuté 146 rapports \\
du NIK
\end{tabular} \\
\hline $\begin{array}{l}\text { Soumis au } \\
\text { Président }\end{array}$ & Oui & Oui & Oui & Oui & Oui & Oui & \\
\hline $\begin{array}{l}\text { Soumis au } \\
\text { Président de la } \\
\text { commission } \\
\text { spécialisée }\end{array}$ & Oui & Oui & Oui & Oui & Oui & Oui & \\
\hline $\begin{array}{c}\text { Copies envoyées } \\
\text { aux autres } \\
\text { commissions } \\
\text { concernées }\end{array}$ & Oui & Oui & Oui & Oui & Oui & Oui & \\
\hline $\begin{array}{c}\text { Notes de synthèse } \\
\text { destinées à la } \\
\text { commission } \\
\text { parlementaire }\end{array}$ & Oui & Oui & Oui & Oui & Oui & Oui & \begin{tabular}{|c|} 
Les résultats d'audit \\
ont été présentés \\
pendant les \\
réunions des \\
commissions \\
parlementaires \\
compétentes
\end{tabular} \\
\hline $\begin{array}{c}\text { Résumés soumis } \\
\text { au Parlement }\end{array}$ & Non & Non & Non & Non & Non & Non & \\
\hline
\end{tabular}




\section{3) Procédures parlementaires utilisées dans le traitement des rapports de I'ISC}

Les procédures régissant les rapports de I'ISC sont fixées par les Règles de procédure du Parlement :

- Le Parlement dispose d'une Commission de contrôle de l'État présidée par un membre de l'opposition ou du parti au gouvernement (découlant de la parité dans la répartition des commissions au Parlement).

- La Commission ne nomme pas de rapporteur.

- Des auditions sont organisées durant lesquelles sont examinés les rapports. Après discussion,

la Commission transmet au gouvernement des opinions ou des recommandations visant à éliminer les irrégularités.

- D'autres commissions analysent et discutent les rapports soumis.

Le Parlement possède une procédure de décharge établie. Cette dernière est réglementée par les chapitres de la Constitution selon lesquels le Parlement octroie sa décharge sur l'exécution du budget de l'État pour l'année antérieure, en joignant l'analyse du NIK sur l'exécution du budget de l'État.

\section{Personnel}

La Commission de contrôle de l'État comprend 17 parlementaires, dont un président et trois présidents adjoints.

\section{Participation de I'ISC à l'examen de ses rapports par le Parlement}

Le Président et la direction du NIK participent aux travaux de la Commission. Ils assistent aux réunions où sont débattus les rapports du NIK.

\section{4) Rapports de I'ISC}

Le NIK se concentre sur les recommandations génériques et systémiques dans ses rapports d'audit qui sont également soumis au Parlement. Par ailleurs, les rapports traitent souvent des systèmes de contrôle internes. Tous les rapports comprennent des opinions du NIK ainsi que des recommandations complètes qui doivent être mises en œuvre afin d'éliminer les irrégularités révélées par les audits.

\section{5) Suivi des rapports de l'ISC}

Le NIK surveille l'exécution des recommandations, par exemple en réalisant des audits de suivi qui vérifient si les changements nécessaires ont été mis en place.

\section{6) Audits réalisés à la demande du Parlement}

Les Règles de procédure du Parlement et la Loi relative au Bureau supérieur d'audit permet au Parlement de solliciter des audits.

\section{7) Programme de travail}

Le NIK présente son plan de travail annuel à la réunion de la Commission de contrôle de l'État. Suite aux discussions, aux explications et aux suggestions soumises, le plan de travail est adopté et accepté par la Commission. Les demandes d'audit du Parlement sont acceptées si des sujets similaires n'ont pas été préalablement contrôlés. 


\section{8) Niveau et fréquence des échanges}

Des échanges à la fois formels et informels ont lieu régulièrement avec les commissions parlementaires. Deux employés de la Division du NIK chargée des services généraux est responsable des relations courantes avec le Parlement.

\section{9) Bonnes pratiques}

Le NIK prépare des propositions de textes juridiques sur mesure et suggère comment modifier la législation dans le but de mettre en œuvre ses conclusions et ses recommandations. La division juridique du NIK rédige des projets de modification et suit leur avancement au Parlement.

\section{0) Perspective}

Le Parlement n'a pas l'obligation juridiquement contraignante de mettre en œuvre les propositions faites par le NIK en matière de nouvelle législation. 


\section{PORTUGAL}

Cour des comptes

\section{1) Cadre juridique}

La Constitution portugaise stipule que la Cour des comptes (Tribunal de Contas) est un organe souverain. En conséquence, la Cour est indépendante du Parlement et de toute autre institution.

Il existe néanmoins des formes de coopération entre ces deux institutions.

En vertu de la Constitution, le Président du Parlement reçoit du Président de la Cour le compte général de l'État accompagné du rapport et de l'opinion de la Cour qui s'y rapportent.

La Loi relative à l'organisation et à la procédure de la Cour des comptes stipule que le Parlement peut demander à la Cour de procéder au contrôle d'entités ou de domaines spécifiques et de lui envoyer les rapports d'audit existants.

Le Parlement peut solliciter de la Cour les informations qu'elle a obtenues pendant son suivi de l'exécution du budget de l'État, des rapports sur les résultats de ce contrôle et les clarifications permettant d'évaluer le budget de l'État et le compte général de l'État.

Le rapport d'activité annuel de la Cour est envoyé au Président de la République, au Parlement, au gouvernement et aux organes gouvernementaux des régions autonomes de Madère et des Açores.

Entre dans les attributions de la Cour l'approbation des avis juridiques demandés par le Parlement ou le gouvernement sur les projets de loi visant le domaine financier.

La Cour émet une opinion sur le compte annuel du Parlement. Elle envoie pour information son compte annuel au Parlement.

Remarque : il est important de préciser que la Cour diffuse tous ses rapports par différents moyens.

\section{2) Modalités de soumission des rapports de l'ISC au parlement}

La Cour peut envoyer au Parlement des rapports de toute nature.

Le Parlement peut demander à la Cour de lui soumettre tout type de rapport. Comme cité précédemment, le rapport d'activité annuel de la Cour est envoyé au Parlement, ainsi qu'au Président de la République, au gouvernement et aux organes gouvernementaux des régions autonomes de Madère et des Açores.

Les rapports sont adressés au Président du Parlement et / ou aux présidents des commissions spécialisées. L'envoi des rapports est lié aux résultats des audits.

L'envoi des rapports au Parlement n'est soumis à aucun calendrier ou délai.

\section{Procédures d'envoi des rapports au Parlement}

Il a été instauré des procédures spécifiques, qui sont souples. 
Nombre et type de rapports

\begin{tabular}{|c|c|c|c|c|c|c|c|}
\hline & $\begin{array}{l}\text { Rapports } \\
\text { d'audit } \\
\text { financier }\end{array}$ & $\begin{array}{l}\text { Rapports d'audit } \\
\text { de conformité }\end{array}$ & $\begin{array}{l}\text { Rapports d'audit } \\
\text { de performance }\end{array}$ & $\begin{array}{l}\text { Autres rapports } \\
\text { ou documents }\end{array}$ & $\begin{array}{l}\text { Rapports } \\
\text { d'activité } \\
\text { annuels }\end{array}$ & $\begin{array}{l}\text { Rapports } \\
\text { demandés } \\
\text { par le } \\
\text { Parlement }\end{array}$ & Remarques \\
\hline $\begin{array}{c}\text { Nombre de } \\
\text { rapports soumis }\end{array}$ & & & & & & & 24 (total) \\
\hline $\begin{array}{l}\text { Nombre de } \\
\text { rapports } \\
\text { discutés par le } \\
\text { Parlement }\end{array}$ & & & & & & & \begin{tabular}{|} 
La Cour présente (sans \\
les discuter) ses \\
rapports aux \\
commissions \\
spécialisées. Les \\
rapports de la Cour sont \\
pris en considération \\
par le Parlement
\end{tabular} \\
\hline $\begin{array}{c}\text { Soumis au } \\
\text { Président }\end{array}$ & & & & & & & Oui \\
\hline $\begin{array}{l}\text { Soumis au } \\
\text { Président de la } \\
\text { commission } \\
\text { spécialisée }\end{array}$ & & & & & & & Oui \\
\hline \begin{tabular}{|c|} 
Copies envoyées \\
aux autres \\
commissions \\
concernées
\end{tabular} & & & & & & & Oui \\
\hline $\begin{array}{c}\text { Notes de } \\
\text { synthèse } \\
\text { destinées à la } \\
\text { commission } \\
\text { parlementaire }\end{array}$ & & & & & & & - \\
\hline $\begin{array}{c}\text { Résumés soumis } \\
\text { au Parlement }\end{array}$ & & & & & & & $\begin{array}{c}\text { Les rapports englobent } \\
\text { les synthèses de } \\
\text { direction }\end{array}$ \\
\hline
\end{tabular}

3) Procédures parlementaires utilisées dans le traitement des rapports de l'ISC

Les rapports de la Cour ont pour but d'informer le Parlement et de l'assister dans l'appréciation et le débat des sujets liés aux entités ou domaines contrôlés.

Dans le cadre du traitement des rapports de I'ISC, il n'y a pas de procédures établies dans les Règles de procédure du Parlement. 
II n'y a pas de commission dédiée au traitement des rapports de I'ISC. La majorité des rapports sont envoyés à la Commission du budget et des finances.

- Il n'y a pas d'auditions auxquelles serait conviée l'entité contrôlée ou d'autres personnes concernées afin d'apporter des clarifications ou un complément d'informations.

Outre la commission spécialisée, d'autres commissions ont également la faculté d'examiner les rapports de la Cour.

Aux termes de la Constitution, le Parlement octroie la décharge sur le compte général de l'État en examinant le rapport d'audit et l'opinion de la Cour des comptes.

En règle générale, les actes de la Cour des comptes portugaise (Tribunal de Contas), y compris les rapports d'audit, ne sont pas soumis aux délibérations du Parlement ou à ses commissions. Toutefois, concernant le rapport et l'opinion de la Cour sur le compte général de l'État, un rapporteur est nommé pour rédiger un rapport sur les documents de la Cour examinés pendant la discussion du compte par la Commission du budget et des finances, avant l'adoption du compte par le Parlement.

\section{Personnel}

Les commissions parlementaires sont assistées par des experts, la Commission du budget et des finances dispose d'une unité de services d'assistance technique.

\section{Participation de l'ISC à l'examen de ses rapports par le Parlement}

Lorsque des thématiques liées au contenu des rapports d'audit sont débattues, le Parlement convie normalement la Cour à assister aux réunions.

\section{4) Rapports de I'ISC}

En règle générale, les systèmes de contrôle internes sont minutieusement examinés et évalués au cours des audits réalisés par la Cour. Les rapports d'audit contiennent des conclusions et des opinions claires.

\section{5) Suivi des rapports de l'ISC}

La Cour procède au suivi systématique de l'acceptation de ses recommandations et de la manière dont elles sont mises en œuvre; son rapport et son opinion sur le compte général de l'État sus-cité comprennent un chapitre consacré aux résultats du suivi. Lors du contrôle d'un organisme auquel des recommandations ont été faites dans un précédent rapport, le respect des recommandations est toujours vérifié.

Les rapports de la Cour ne sont pas repris dans les débats annuels sur les projets de budget.

La Cour est caractérisée par une autre forme de suivi selon laquelle, lorsque des irrégularités ou des faits engageant la responsabilité sont révélés, la Chambre juridictionnelle de la Cour a le pouvoir de juger les dirigeants responsables.

\section{6) Audits réalisés à la demande du Parlement}

Des dispositions prévues par la loi permettent au Parlement de solliciter des rapports d'audit à la Cour ainsi que l'exécution d'audits sur des entités ou des problématiques spécifiques. La fréquence de ces requêtes est liée aux besoins d'assistance du Parlement et, par conséquent, elle est variable. La Cour est 
tenue par la loi de réaliser deux audits par an. Si le Parlement émet d'autres demandes, elle les analyse dans le contexte de son programme de contrôle annuel. En règle générale, la Cour s'efforce de donner suite à ces sollicitations.

\section{7) Programme de travail}

La Cour ne consulte pas le Parlement sur son programme de travail annuel en matière de contrôle.

\section{8) Niveau et fréquence des échanges}

Des échanges entre la Cour et le Parlement ont lieu toutes les fois que des affaires pertinentes doivent être examinées, sans suivre de calendrier défini. Les relations administratives sont de la responsabilité des divisions compétentes de la Cour, sous la coordination de son Président et Directeur général.

\section{9) Bonnes pratiques}

Nous estimons que nos relations avec le Parlement sont relativement formalisées, avec la volonté de préserver l'indépendance de la Cour, mais parallèlement, elles sont extrêmement collaboratives et fructueuses. 


\section{ROUMANIE}

Cour des comptes

\section{1) Cadre juridique}

La Cour des comptes roumaine est une autorité centrale spécialisée et autonome, régie par quatre titres de la Constitution roumaine, révisée et publiée à nouveau en 2003. L'Article $N^{\circ} 140$ de la Constitution stipule :

1. La Cour des comptes exerce le contrôle sur le mode de formation, d'administration et d'utilisation des ressources financières de l'État et du secteur public. Dans les conditions fixées par la loi organique, les litiges résultant de l'activité de la Cour des comptes sont jugés par les instances judiciaires spécialisées.

2. La Cour des comptes présente annuellement au Parlement un rapport sur les comptes de gestion du budget public national de l'exercice budgétaire expiré, comprenant aussi les irrégularités constatées.

3. À la demande de la Chambre des députés et du Sénat, la Cour des comptes contrôle le mode de gestion des ressources publiques et présente un rapport sur la situation constatée.

4. Les conseillers aux comptes sont nommés par le Parlement pour un mandat de neuf ans, qui ne peut pas être prolongé ou renouvelé. Les membres de la Cour des comptes sont indépendants dans l'exercice de leur mandat et sont inamovibles pour toute sa durée. Ils sont soumis aux incompatibilités prévues par la loi pour les juges.

5. La Cour des comptes est renouvelée par un tiers des conseillers aux comptes nommés par le Parlement, tous les trois ans, dans les conditions prévues par la loi organique de la Cour.

6. Les membres de la Cour des comptes sont révoqués par le Parlement, dans les cas et les conditions prévus par la loi.

Le cadre juridique pour l'exécution d'activités spéciales est déterminé par la Loi Nº4/1992 relative à l'organisation et au fonctionnement de la Cour des comptes, publiée à nouveau, accompagnée de ses amendements et compléments ultérieurs.

Conformément à la Loi, les principales obligations de la Cour des comptes envers le Parlement sont les suivantes :

- La Cour des comptes soumet annuellement au Parlement un rapport sur les comptes de gestion du budget public national de l'exercice budgétaire expiré, comprenant aussi les irrégularités constatées (Article 4 alinéa 1).

- La Cour des comptes peut soumettre au Parlement ou bien, par l'intermédiaire des chambres des comptes des comtés, aux autorités publiques des unités administratives et territoriales, des rapports portant sur les domaines placés sous leur juridiction, chaque fois que la Cour le juge nécessaire (Article 40).

- Chaque fois que la Cour des comptes le juge nécessaire, elle soumet au Parlement et, par l'intermédiaire des chambres des comptes des comtés et de la municipalité de Bucarest, au 
conseil municipal de Bucarest et aux conseils locaux et des comtés, des rapports portant sur des sujets relevant de sa juridiction (Article 4 alinéa 3).

- La Cour des comptes entérine, à la demande du Sénat ou de la Chambre des députés, le projet de budget de l'État ainsi que les avant-projets de loi relatifs aux finances et à la comptabilité publiques ou celles dont l'application entraînerait une diminution des revenus ou une augmentation des dépenses approuvées par la loi budgétaire (Article 41).

- À la demande de la Chambre des députés ou du Sénat, la Cour des comptes examine la gestion des ressources publiques et rend compte de ses constatations (Article 4 alinéa 2).

- Les contrôles exercés par la Cour des comptes sont initiés ex officio et peuvent être arrêtés uniquement par le Parlement et exclusivement lorsque les pouvoirs définis par la loi ont été dépassés (Article 3 alinéa 2).

- Les audits demandés par la Chambre des députés ou du Sénat, dans le domaine de compétence de la Cour, sont obligatoires. Aucune autre autorité publique ne peut formuler une telle demande à la Cour (Article 3 alinéa 3).

- L'exécution des budgets de la Chambre des députés, du Sénat, de l'administration présidentielle, du gouvernement, de la Haute Cour de cassation et de justice, de la Cour constitutionnelle, du Conseil législatif et du défenseur du peuple est exclusivement contrôlée par la Cour des comptes (Article 25).

- Le budget de la Cour des comptes est contrôlé par une commission établie à ces fins par les deux chambres parlementaires (Article 25 alinéa 2).

La Loi $\mathrm{N}^{\circ} 500 / 2002$ relative aux finances publiques, avec ses amendements et compléments ultérieurs, stipule à l'Article 56 alinéa 5 : Le compte général annuel de l'exécution du budget de l'État, du budget de l'assurance sociale publique et des autres comptes d'exécution annuels sont approuvés par la loi après avoir été vérifiés par la Cour des comptes.

\section{2) Modalités de soumission des rapports de l'ISC au parlement}

En vertu de l'Article 140 de la Constitution roumaine, la Cour des comptes rend annuellement compte au Parlement des comptes de gestion du budget public national dans la dernière année budgétaire complète, comprenant aussi les irrégularités constatées.

La Loi $n^{\circ}$ 94/1992 stipule :

- La Cour des comptes soumet annuellement au Parlement un rapport sur les comptes de gestion du budget public national de l'exercice budgétaire clôturé, comprenant aussi les irrégularités constatées (Article 4 alinéa 1).

- La Cour des comptes peut soumettre au Parlement ou bien, par l'intermédiaire des chambres des comptes des comtés, aux autorités publiques des unités administratives et territoriales, des rapports portant sur les domaines relevant de sa juridiction, chaque fois que la Cour le juge nécessaire (Article 40).

- Chaque fois que la Cour des comptes le juge nécessaire, elle soumet au Parlement et, par l'intermédiaire des chambres des comptes des comtés et de la municipalité de Bucarest, au conseil municipal de Bucarest et aux conseils locaux et des comtés, des rapports portant sur des sujets relevant de sa juridiction (Article 4 alinéa 3). 
- À la demande de la Chambre des députés ou du Sénat, la Cour des comptes examine la gestion des ressources publiques et rend compte de ses constatations (Article 4 alinéa 2).

- Les audits demandés par la Chambre des députés ou du Sénat, dans le domaine de compétence de la Cour, sont obligatoires. Aucune autre autorité publique ne peut formuler une telle demande à la Cour (Article 3 alinéa 3).

- Dans les six mois suivant la réception des comptes, prévus à l'Article 26, des organismes compétents pour les ouvrir et les transmettre, la Cour des comptes rédige le rapport public annuel puis le soumet au Parlement (Article 38 alinéa 1).

- Le rapport public annuel comprend : les observations de la Cour des comptes sur les comptes d'exécution des budgets soumis à ses contrôles, les conclusions issues des audits commandés par la Chambre des députés ou le Sénat, ou bien effectués sur des autorités administratives autonomes, des sociétés commerciales dont le capital est détenu intégralement ou en majeure partie par l'État, et d'autres entités juridiques relevant du périmètre de contrôle de la Cour, les infractions constatées et les mesures prises pour contraindre les responsables à rendre compte, d'autres sujets que la Cour jugera utile (Article 39).

Comme sus-cité, le rapport le plus important soumis par la Cour des comptes au Parlement est le rapport public annuel sur les comptes de gestion du budget public national de l'exercice budgétaire clôturé. Ce rapport contient essentiellement les résultats d'un grand nombre d'audits financiers de comptes publics, mais également des audits de contrôle (conformité) et de performance. Il est envoyé, par les organes qui en sont responsables et les transmettent, au Parlement dans les six mois à compter de la réception des comptes prévus à l'Article 26 de la Loi.

La Cour des comptes peut soumettre au Parlement, pour information, les rapports relevant de sa juridiction, chaque fois que la Cour le juge nécessaire. La Cour a soumis au Parlement :

- Des résumés de rapports d'audit de performance et de rapports d'audit spéciaux portant sur différents sujets ;

- Des résumés d'audit financiers individuels sur les principales autorisations de dépenses ;

- Des résumés de rapport d'audit financiers sur l'exécution des comptes des budgets soumis aux autorités administratives autonomes.

Le rapport public annuel est soumis aux présidents des deux chambres parlementaires, aux présidents des commissions permanentes du budget, des finances et des banques du Sénat et de la Chambre des députés, aux membres de ces commissions, et à tous les parlementaires. Il est envoyé au Parlement accompagné du rapport d'activité annuel de la Cour des comptes.

Ce rapport est transmis par les organes responsables au Parlement dans les six mois suivant la réception des comptes prévus à l'Article 26 de la Loi.

\section{Procédures d'envoi des rapports au Parlement}

La Cour des comptes envoie ses rapports au Parlement dans le cadre d'une procédure spécifique, en commençant par l'Article 58 de la Loi 94/1992 qui stipule :

Ce qui suit énoncent les pouvoirs et les obligations de la Cour des comptes réunie en plénière :

$\mathrm{m}$ ) approbation des rapports à soumettre au Parlement. 
La Cour des comptes a adopté des réglementations internes qui définissent les procédures d'élaboration du rapport public annuel et d'autres rapports qu'elle soumet au Parlement et aux autorités publics des unités administratives et territoriales.

\section{Nombre et type de rapports}

\begin{tabular}{|c|c|c|c|c|c|c|c|}
\hline & $\begin{array}{l}\text { Rapports } \\
\text { d'audit } \\
\text { financier }\end{array}$ & $\begin{array}{c}\text { Rapports d'audit } \\
\text { de conformité }\end{array}$ & $\begin{array}{l}\text { Rapports d'audit } \\
\text { de performance }\end{array}$ & \begin{tabular}{|c|} 
Autres rapports \\
ou documents
\end{tabular} & $\begin{array}{l}\text { Rapports } \\
\text { d'activité } \\
\text { annuels }\end{array}$ & $\begin{array}{c}\text { Rapports } \\
\text { demandés par } \\
\text { le Parlement }\end{array}$ & Remarques \\
\hline $\begin{array}{c}\text { Nombre de } \\
\text { rapports soumis }\end{array}$ & 5 & - & 6 & $\begin{array}{c}1 \text { rapport public } \\
\text { pour } 2014+1 \\
\text { rapport spécial }\end{array}$ & 1 & - & \\
\hline $\begin{array}{c}\text { Nombre de } \\
\text { rapports discutés } \\
\text { par le Parlement }\end{array}$ & - & - & - & - & - & - & \\
\hline $\begin{array}{l}\text { Soumis au } \\
\text { Président }\end{array}$ & 5 & - & 6 & $\begin{array}{c}1 \text { rapport public } \\
\text { pour } 2014+1 \\
\text { rapport spécial }\end{array}$ & 1 & - & \\
\hline $\begin{array}{c}\text { Soumis au } \\
\text { Président de la } \\
\text { commission } \\
\text { spécialisée }\end{array}$ & 5 & - & 6 & $\begin{array}{l}\text { 1rapport public } \\
\text { pour } 2014+1 \\
\text { rapport spécial }\end{array}$ & 1 & - & \\
\hline $\begin{array}{c}\text { Copies envoyées } \\
\text { aux autres } \\
\text { commissions } \\
\text { concernées }\end{array}$ & 5 & - & 6 & 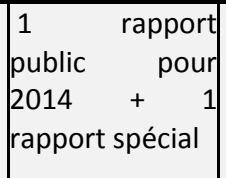 & 1 & - & \\
\hline $\begin{array}{c}\text { Notes de synthèse } \\
\text { destinées à la } \\
\text { commission } \\
\text { parlementaire }\end{array}$ & - & - & - & - & - & - & \\
\hline $\begin{array}{c}\text { Résumés soumis au } \\
\text { Parlement }\end{array}$ & 5 & - & 6 & $\begin{array}{c}1 \text { rapport public } \\
\text { pour } 2014+1 \\
\text { rapport spécial }\end{array}$ & - & - & \\
\hline
\end{tabular}

\section{3) Procédures parlementaires utilisées dans le traitement des rapports de l'ISC}

L'Article 13a de la Réglementation sur les activités conjointes de la Chambre des députés et du Sénat du Parlement énonce des dispositions relatives à la Cour des comptes :

La Chambre des députés et le Sénat se réunissent en séance commune pour :

2. approuver le budget de l'État et le budget des assurances sociales de l'État, amender les lois et approuver le compte d'exécution du budget ; 
7. examiner les rapports du Conseil supérieur de la défense nationale et de la Cour des comptes ;

20. nommer les membres de la Cour des comptes.

Les commissions parlementaires sont des organes de travail des chambres du Parlement; à ce titre, elles jouent un rôle important dans la préparation des procédures, et elles exercent des fonctions parlementaires, notamment au regard des questions législatives et de contrôle.

Dans le système parlementaire roumain, conformément à la Constitution, les chambres peuvent créer des commissions permanentes, des commissions d'enquête provisoires ou des commissions spéciales. De même, des commissions conjointes peuvent être établies pour la Chambre des députés et le Sénat. Toutes ces commissions reflètent la configuration politique de chaque chambre.

La Chambre des députés a une commission permanente dédiée au budget, aux finances et aux banques, avec le champ d'activité suivant : le budget de l'État et l'exécution du budget, le budget des assurances sociales de l'État, les taxes relevant de la politique financière et taxes douanières, l'assurance et la réassurance, l'équilibre monétaire, la circulation monétaire, les crédits et le système de crédit, les intérêts, les ristournes ; l'activité boursière et les instruments commerciaux ; les prêts externes proposés ou garantis par l'État ; les investissements provenant des crédits budgétaires. Cette commission comprend 33 membres (députés).

Le Sénat a une commission permanente similaire destinée au budget, aux finances et aux banques, qui comprend 11 sénateurs.

Ces deux commissions permanentes examinent le rapport public annuel en séance commune, et elles débattent des avant-projets de loi portant sur l'exécution du budget annuel de l'État et du budget des assurances sociales de l'État (présentés par le Gouvernement à l'approbation du Parlement). Les commissions peuvent analyser d'autres rapports reçus indépendamment de la Cour des comptes.

La Loi 94/1992 accorde à ces deux commissions permanentes les compétences suivantes :

- Article 46, alinéa 1 : Les membres de la Cour des comptes sont nommés par le Parlement, sur proposition des commissions permanentes du budget, des finances et des banques des deux chambres, pour un mandat de neuf ans, qui ne peut pas être prolongé ou renouvelé ;

- Article 50, alinéa 3 : La révocation des membres de la Cour des comptes est appliquée par le Parlement, sur proposition des commissions spécialisées prévues à l'Article 46 alinéa 1.

La composition du bureau des deux commissions du budget, des finances et des banques est établie, dans les deux cas, de manière autonome, en fonction du paysage politique des chambres, et des négociations entre les représentants des groupes parlementaires.

Les deux commissions permanentes du budget, des finances et des banques remettent en séance plénière du Parlement un rapport conjoint concernant les avant-projets de loi sur l'exécution du budget de l'État et l'exécution du budget des assurances sociales de l'État, en utilisant le rapport public annuel de la Cour des comptes, en vertu de la Loi 500/2002 relative au finances publiques, Article 56 alinéa 5 : « Le compte général annuel de l'exécution du budget de l'État, du budget des assurances sociales de l'État et d'autres comptes d'exécution sont approuvés par la loi après vérification par la Cour des comptes ». Ce rapport conjoint est le résultat des travaux des deux commissions, pas de rapporteurs individuels (parlementaires). 
La Cour des comptes soumet au Parlement le rapport public annuel sur les comptes de gestion du budget public national de l'exercice budgétaire clôturé.

La Cour des comptes peut soumettre au Parlement, pour information, des rapports sur des domaines relevant de sa juridiction, chaque fois que la Cour le juge nécessaire.

Chaque fois que les commissions permanentes des deux chambres du Parlement le jugent utile, elles peuvent inviter des représentants de la Cour à apporter des clarifications ou un complément d'informations sur ces résumés.

Le rapport public annuel est présenté au Parlement réuni en séance plénière pendant le débat sur les avant-projets de loi pendant lequel le Parlement approuve les comptes d'exécution du budget de l'État et du budget des assurances sociales de l'État, en vertu de l'Article 13 de la Réglementation des activités conjointes de la Chambre des députés et du Sénat:

La Chambre des députés et le Sénat tiennent des réunions communes pour :

2. approuver le budget de l'État et le budget des assurances sociales de l'État, amender les lois et approuver le compte d'exécution budgétaire ;

7. examiner les rapports du Conseil supérieur de la défense nationale et de la Cour des comptes.

Les rapports de la Cour des comptes soumis à la Chambre des députés ou au Sénat, conformément à I'Article 140, alinéa 3 de la Constitution ${ }^{42}$ et de l'Article 4, alinéa 2 de la Loi 94/1992 ${ }^{43}$, sont débattus en séance plénière de la Chambre du Parlement qui a sollicité l'audit.

Des rapports résumés sont envoyés aux commissions permanentes pour information, essentiellement aux commissions permanentes du budget, des finances et des banques des deux chambres, en vertu de l'Article 4, alinéa 3 de la Loi $94 / 1992^{44}$, de manière à : analyser, en commission séparée, les rapports résumés des audits de performance et des audits spéciaux portant sur des thèmes divers, les résumés des audits financiers indépendants menés sur les principales autorisations de dépenses, les résumés des audits financiers examinant l'exécution des comptes des budgets soumis à des autorités administratives autonomes, ainsi que différents rapports.

Outre la soumission de rapports aux commissions permanentes du budget, des finances et des banques, la Cour des comptes envoie des rapports résumés à d'autres commissions permanentes, pour information, en fonction des thèmes et des domaines qui relèvent de la juridiction de ces commissions. Tous ces rapports résumés sont analysés uniquement en commission et pas en séance plénière de la Chambre des députés et du Sénat.

"At the request of the Chamber of Deputies or the Senate, the Court of Accounts shall check the management of public resources, and report on its findings."

"Upon request by the Chamber of Deputies or by the Senate, the Court of Accounts shall examine the public resources management and shall report on the findings."

"Whenever the Court of Accounts deems it necessary, it shall submit to the Parliament and, through the county and Bucharest Municipality chambers of accounts, to local, county and Bucharest Municipality councils, reports in the fields under its jurisdiction." 
Les avant-projets de loi et les propositions législatives sont soumis pour débat à la Chambre des députés ou au Sénat. Cette procédure s'applique également aux deux textes à approuver du compte d'exécution du budget de l'État et du budget des assurances sociales de l'État débattus en séances communes de la Chambre des députés et du Sénat.

Une fois que les deux projets de loi ont été remis par le gouvernement et enregistrés, le bureau permanent conjoint de la Chambre des députés et du Sénat les distribue aux parlementaires et les envoie aux commissions permanentes du budget, des finances et des banques pour examen en séance commune, pour avoir un avis consultatif.

Pour rédiger les rapports conjoints destinés à la séance plénière du Parlement, les commissions permanentes du budget, des finances et des banques doivent analyser le rapport public annuel de la Cour des comptes.

Les rapports conjoints comportent les conclusions et les constatations que la Cour des comptes a formulées dans son rapport annuel, par exemple : les observations de la Cour des comptes sur les comptes d'exécution des budgets soumis à ses contrôles, les conclusions issues des audits commandés par la Chambre des députés ou le Sénat, ou bien effectués sur des autorités administratives autonomes, des sociétés commerciales dont le capital est détenu intégralement ou en majeure partie par l'État, et d'autres entités juridiques relevant du périmètre de contrôle de la Cour, les infractions constatées et les mesures prises pour contraindre les responsables à rendre compte, ainsi que d'autres sujets que la Cour juge utile.

Une fois les rapports conjoints finalisés, les deux avant-projets de loi destinés à l'adoption des comptes d'exécution du budget de l'État et du budget des assurances sociales de l'État sont débattus en séance plénière.

Les rapports conjoints des deux commissions permanentes du budget, des finances et des banques sont publiés sur le site Internet du Parlement.

Les avant-projets de loi pour lesquels les commissions compétentes ont rédigé des rapports conjoints sont mis à l'ordre du jour du Parlement.

Si les avant-projets de loi sont adoptés, ils sont signés par les présidents des deux chambres puis ils sont envoyés au Président de la Roumanie qui les promulguera.

Les lois entrent en vigueur trois jours après leur parution au Journal officiel de Roumanie.

\section{Personnel}

Le personnel des deux commissions permanentes se compose de fonctionnaires parlementaires, qui sont rattachés au Secrétariat général, selon l'organigramme interne. Le fonctionnement des commissions permanentes et le nombre de fonctionnaires qui y sont affectés sont définis par la réglementation interne des commissions, qui n'est pas rendue publique.

Les commissions permanentes peuvent convier des personnes intéressées, ainsi que des spécialistes au nom de certaines autorités publiques ou d'autres institutions spécialisées, à participer à leurs séances. 


\section{Participation de I'ISC à l'examen de ses rapports par le Parlement}

L'ISC ne participe pas à la préparation des auditions aux côtés de la commission ou du Parlement.

Le rapport public annuel est présenté en séance plénière du Parlement pendant le débat sur les avantprojets de loi destinés à approuver les comptes d'exécution du budget de l'État et du budget des assurances sociales de l'État, en vertu de l'Article 13 de la Réglementation des activités conjointes de la Chambre des députés et du Sénat. À cette occasion, la Cour des comptes est représentée au plus haut niveau, par son Président ou son vice-président, qui présente un résumé du rapport public annuel au Parlement réuni en séance plénière.

Lorsque les rapports résumés sont envoyés aux commissions permanentes pour information, essentiellement aux commissions permanentes du budget, des finances et des banques des deux chambres, les présidents des commissions peuvent inviter le président ou les vice-présidents de la Cour ou les conseilleurs aux comptes (membres de la séance plénière de la Cour) à assister à la séance et à présenter les résultats des travaux réalisés par la Cour. D'autres collaborateurs peuvent assister aux réunions, dont les premiers responsables de la Cour, les directeurs et les autres responsables spécialistes des thématiques contenues dans les rapports résumés.

\section{4) Rapports de I'ISC}

En vertu de l'Article 39 de la Loi 94/1992, le rapport public annuel de la Cour des comptes doit impérativement porter sur : les observations de la Cour des comptes sur les comptes d'exécution des budgets soumis à ses contrôles, les conclusions issues des audits commandés par la Chambre des députés ou le Sénat, ou bien effectués pour des autorités administratives autonomes, des sociétés commerciales dont le capital est détenu intégralement ou en majeure partie par l'État, et d'autres entités juridiques relevant du périmètre de contrôle de la Cour, les infractions constatées et les mesures prises pour contraindre les responsables à rendre compte, et d'autres sujets que la Cour juge utile.

En outre, le rapport public annuel contient un chapitre qui met en avant l'évaluation du système de gestion / contrôle interne du secteur public. Ce chapitre présente les principales constatations sur la mise en œuvre des exigences réglementaires, en matière de contrôle de la gestion interne, par les directeurs des organes publics investis de cette fonction.

En vertu de I'Article 32, alinéa 2 de la Loi 94/1992, les contrôleurs publics externes désignés pour procéder à la vérification des comptes, ainsi qu'à d'autres activités qui relèvent des compétences de la Cour des comptes, rédigent des rapports dans lesquels figurent les constatations et les conclusions de l'audit, les mesures recommandées et l'opinion émise, conformément aux procédures fixées par le règlement, approuvée en conformité avec l'Article 11 alinéa $2^{45}$. Accounts, as well as the follow up of the documents resulting from these activities, shall be performed in keeping with the regulation approved by the plenum of the Court of Accounts, in accordance with this law." 


\section{5) Suivi des rapports de l'ISC}

La Cour des comptes exécute sa mission de manière autonome. Elle réalise trois types de contrôle : l'audit financier, l'audit de performance et l'audit de conformité (contrôle thématique) présentés de manière détaillée dans le rapport public annuel.

L'Article 33 de la Loi 94/1992 stipule que « le suivi des rapports d'audit est exécuté en conformité avec le règlement adopté selon les dispositions de l'Art. 11 alinéa $2^{46}$. Si les comptes présentent des irrégularités, un certificat de conformité est publié et adressé à l'organe contrôlé. Si les comptes révèlent des déviations en matière de légalité et de régularité, qui ont entraîné des préjudices, les dirigeants de l'organe public contrôlé en sont informés. La direction de l'organe contrôlé est responsable de déterminer l'étendue des préjudices et d'ordonner des actions pour pallier ces préjudices. Si les rapports d'audit révèlent des signes d'infraction pénale, le responsable de division en informe les institutions concernées pour assurer le suivi des constatations, ainsi que l'organe contrôlé. Lorsqu'il s'avère impossible de rédiger un rapport d'audit, l'organe contrôlé est enjoint de compléter et / ou refaire les comptes examinés $"$.

En vertu des dispositions de l'Article 35 : "L'organe contrôlé peut, dans les quinze jours suivant la réception du rapport, formuler par écrit des objections aux constatations présentées dans les rapports établis par des contrôleurs publics externes. Ces objections sont soumises au dirigeant de l'institution de contrôle et prises en compte dans le suivi des constatations. »

L'instrument principal servant à mesurer l'influence des travaux de la Cour des comptes sur la qualité de la gestion des fonds et des biens publics est le rapport de suivi. Ce rapport a pour objet de vérifier la façon dont les organes contrôlés :

- Ont mis en œuvre les mesures exigées sur décision de la Cour de comptes ;

- Ont mis en œuvre les recommandations résultant de l'audit de performance.

Le Parlement ne prend pas en compte la mise en œuvre des recommandations de I'ISC contenues dans ses rapports d'audit ou les mécanismes qu'elle utilise à ces fins.

Le rapport public annuel contient un chapitre qui met en avant « l'évaluation du système de gestion / contrôle interne » dans le secteur public. Ce chapitre présente les principales constatations sur la mise en œuvre des exigences réglementaires, en matière de contrôle de la gestion interne, par les directeurs des organes publics investis de cette fonction.

Les rapports de I'ISC ne sont pas régulièrement utilisés lors du débat annuel sur les projets de budget.

\section{6) Audits réalisés à la demande du Parlement}

Deux dispositions juridiques permettent au Parlement de solliciter des audits.

Dans la Constitution roumaine, Article 140, alinéa 3 : " À la demande de la Chambre des députés ou du Sénat, la Cour des comptes contrôle le mode de gestion des ressources publiques et présente un rapport sur la situation constatée. » 
Dans la Loi 94/1992, I'Article 4 alinéa 2 et l'Article 3 s'appliquent. L'Article 4 alinéa 2 stipule que "à la demande de la Chambre des députés ou du Sénat, la Cour des comptes examine le mode de gestion des ressources publiques et présente un rapport sur la situation constatée. " L'Article 3, alinéa 3 stipule que "les audits demandés par la Chambre des députés ou le Sénat, dans le domaine de compétence de la Cour, sont obligatoires. Aucune autre autorité publique ne peut formuler une telle demande auprès de la Cour. »

La dernière demande a été émise en 2009 par la Chambre des députés pour vérifier les sommes dépensées par le ministère du Tourisme pour promouvoir l'image et le tourisme roumains.

La dernière demande émise par le Sénat en 2012 concernait l'inclusion dans le programme d'activité de 2012 des audits financiers de six unités administratives et territoriales.

\section{7) Programme de travail}

En vertu de l'Article 3 de la Loi 94/1992 : "La Cour des comptes élabore de manière autonome son programme d'activité. Les contrôles exercés par la Cour des comptes sont initiés ex officio et peuvent être arrêtés uniquement par le Parlement et exclusivement lorsque les pouvoirs définis par la loi ont été dépassés. Les audits demandés par la Chambre des députés ou le Sénat, dans le domaine de compétence de la Cour, sont obligatoires. Aucune autre autorité publique ne peut formuler une telle demande auprès de la Cour. »

\section{8) Niveau et fréquence des échanges}

Il y a régulièrement des échanges entre le Président de la Cour, le Président du Parlement et les présidents des commissions du budget et des finances. Si nécessaire, il y a également des échanges avec les présidents des commissions parlementaires concernées. L'organisation de ces réunions n'est régie par aucune règle stricte. Lorsque la Cour dépose le rapport public annuel devant le Parlement, il est clair que des échanges informels ont lieu, mais également à d'autres occasions. Le Président actuel de la Cour a une solide expérience parlementaire (il a été président du Sénat, la chambre haute du Parlement, pendant huit ans) et, par conséquent, il entretient des relations privilégiées avec les parlementaires.

Dans le cadre du travail, la Cour a des échanges avec le personnel parlementaire, mais elle ne dispose pas d'unité spéciale chargée de cette relation. Ces échanges sont essentiellement entretenus par le directeur du bureau du Président ou / et par le chef de l'unité des relations internationales.

\section{9) Bonnes pratiques}

En règle générale, les Bonnes pratiques demandent d'avoir une PAC spécifique dont la fonction est de s'occuper exclusivement des rapports / constatations de I'ISC. La Roumanie n'a pas encore constitué cette commission. La Cour s'adresse aux commissions compétentes du Sénat et de la Chambre des députés, selon le besoin.

\section{0) Perspective}

La Cour considère que l'établissement d'une PAC dans un avenir proche renforcera ses relations avec le Parlement. Récemment, elle a déjà engagé des discussions avec le Parlement à plusieurs occasions, et elle s'attend à des résultats positifs. 
11) Y-a-t-il d'autres éléments concernant les relations actuelles entre I'ISC et le Parlement que vous souhaiteriez souligner, et quelles a été leur évolution au cours des cinq dernières années?

Nous estimons que les relations entre la Cour et le Parlement s'améliorent continuellement, et que le rôle joué par l'ISC est très apprécié des parlementaires.

\section{SERBIE}

Cour des comptes

\section{1) Cadre juridique}

L'Article 96 de la Constitution de la République de Serbie stipule :

La Cour des comptes est l'organe suprême chargé du contrôle des finances publiques en République de Serbie ; elle est indépendante et soumise au contrôle de l'Assemblée nationale, à laquelle elle rend compte de son action. Une loi sur la Cour des comptes doit être promulguée.

Cet article confirme l'autonomie et l'indépendance de I'ISC, soumise au contrôle de l'Assemblée nationale, à laquelle elle est tenue de rendre compte.

La Loi relative à la Cour des comptes définit I'ISC comme étant l'autorité étatique suprême chargée de vérifier les fonds publics de la République de Serbie, en qualité d'institution d'État autonome et indépendante, qui rend compte des activités relevant de ses attributions et est soumise au contrôle de l'organe législatif, l'Assemblée nationale.

La Partie 5 de la Loi relative à la Cour des comptes définit les obligations de l'ISC en matière de rapports comme suit :

Obligation de rendre compte à l'Assemblée (Article 43) :

L'Institution informe l'Assemblée en soumettant :

1. Le rapport d'activité annuel ;

2. Les rapports spéciaux élaborés pendant l'année ;

3. Le rapport d'audit sur le bilan annuel du budget de la République, les bilans annuels des plans financiers des agences d'assurance obligatoires et les états financiers consolidés de la République.

Le contenu des rapports visés à l'alinéa 1 du présent Article est défini de manière plus détaillée par les Règles de procédure.

Obligation de rendre compte aux assemblées et autorités locales (Article 44) :

L'Institution rend compte auprès des assemblées locales de ses audits réalisées sur des entités dans leur sphère de compétence.

Les rapports visés à l'alinéa 1 du présent Article sont simultanément soumis à l'Assemblée. 
Rapports d'activité annuels de I'Institution (Article 45) :

Avant le 31 mars, I'Institution soumet à l'Assemblée le rapport d'activité annuel de I'Institution pour l'année antérieure.

Rapports spéciaux (Article 46) :

L'Institution est habilitée, en cours d'année, à soumettre à l'Assemblée des rapports spéciaux portant sur des sujets particulièrement importants ou urgents qui, selon le Conseil, ne devraient pas être reportés jusqu'au prochain rapport standard.

L'Institution soumet, à la demande de l'Assemblée, les rapports contenant les informations et les données sollicitées, en conformité avec les dispositions prévues par la loi.

Les rapports portant sur les audits des comptes finaux du budget de l'État, des bilans annuels des plans financiers des agences d'assurance obligatoires et des états financiers consolidés de la République sont présentés à l'Article 47.

Dans le cadre de la procédure d'adoption des comptes finaux de l'État, l'Institution communique à l'Assemblée les résultats des audits sur les comptes finaux du budget de l'État, les bilans annuels des agences d'assurances obligatoires et les états financiers consolidés de l'État, conformément à la loi relative à la réglementation du système budgétaire.

\section{Examen du rapport (Article 48):}

L'instance compétente de l'Assemblée, après examen du rapport élaboré par l'Institution, soumet ses opinions et ses recommandations à l'Assemblée sous la forme d'un rapport.

En s'appuyant sur des faits et des circonstances substantiels indiqués dans les rapports visés à l'alinéa 1 du présent Article, I'Assemblée prend une décision au regard des recommandations proposées, des mesures et des délais de mise en œuvre.

L'Assemblée peut demander à l'Institution de fournir des clarifications supplémentaires sur certains faits et circonstances.

Publicité des travaux (Article 49):

Les travaux de l'Institution sont publics et conformes à la loi et aux Règles de procédure.

L'Institution soumet son rapport d'activité à l'Assemblée pour examen au moins une fois par an.

La Loi relative au système budgétaire (BSL) définit le contrôle externe à l'Article 92 :

Les états financiers annuels de la République de Serbie ainsi que les états financiers annuels des agences chargées de l'assurance sociale obligatoire sont soumis à des contrôles externes, au titre des dispositions prévues par la loi déterminant la juridiction de l'Institution supérieure de contrôle.

Les états financiers annuels des budgets des gouvernements locaux sont également soumis à un contrôle externe. 
Le contrôle visé à l'alinéa 2 du présent Article est exécuté par l'Institution supérieure de contrôle en respectant la loi déterminant la juridiction de l'Institution supérieure de contrôle.

Nonobstant l'alinéa 3 du présent Article, le contrôle externe des budgets des gouvernements locaux peut en outre être exécuté, avec l'accord de l'Institution supérieure de contrôle et la prise en compte de la décision de l'assemblée gouvernementale locale, par une personne qui remplit les conditions liées au contrôle d'états financiers prévues par la loi qui régit les activités de comptabilité et de contrôle.

La Loi relative au financement des partis politiques définit le contrôle exercé par l'ISC à l'Article 34 de la manière suivante :

L'Agence (anti-corruption) peut, après avoir examiné les rapports financiers d'un organe politique, demander à l'Institution supérieure de contrôle de conduire un audit sur ces rapports, en conformité avec les dispositions prévues par la loi qui détermine les compétences de l'Institution supérieure de contrôle.

\section{2) Modalités de soumission des rapports de l'ISC au parlement}

L'ISC serbe envoie au Parlement (I'Assemblée nationale) des audits financiers, des audits de conformité, des audits de performance, ainsi que des rapports (d'activité) annuels.

À une occasion, en se référant à la conclusion de l'Assemblée nationale, après examen de son rapport d'activité annuel, I'ISC a soumis à I'Assemblée nationale un rapport indépendant sur la mise en œuvre des recommandations prononcées dans les rapports d'audit couvrant l'année 2011. Ce rapport comprenait une vue générale des mesures mises en œuvre et contrôlées par I'ISC durant 2013, jusqu'au 25 septembre 2013.

L'ISC rend compte séparément des audits individuels.

Les rapports sont adressés à l'Assemblée nationale dans son ensemble.

Chaque année au 31 mars, I'ISC soumet à l'Assemblée son rapport d'activité annuel concernant l'année antérieure.

\section{Procédures d'envoi des rapports au Parlement}

Lors de la soumission de tous les rapport d'audit à l'Assemblé nationale pour une année donnée, l'ISC informe le Président de la commission spécialisée (Commission des finances, du budget de l'État et du contrôle des dépenses publiques) par courrier officiel que le programme de contrôle de I'ISC de l'année est achevé, et lui demande de programmer une séance de commission pour que I'ISC présente aux parlementaires les principales constatations qu'elle a consignées dans ses rapports d'audit. 
Nombre et type de rapports

\begin{tabular}{|c|c|c|c|c|c|c|c|}
\hline & $\begin{array}{l}\text { Rapports } \\
\text { d'audit } \\
\text { financier }\end{array}$ & $\begin{array}{l}\text { Rapports } \\
\text { d'audit de } \\
\text { conformité }\end{array}$ & $\begin{array}{l}\text { Rapports d'audit } \\
\text { de performance }\end{array}$ & $\begin{array}{l}\text { Autres } \\
\text { rapports ou } \\
\text { documents }\end{array}$ & $\begin{array}{l}\text { Rapports } \\
\text { d'activité } \\
\text { annuels }\end{array}$ & $\begin{array}{c}\text { Rapports } \\
\text { demandés } \\
\text { par le } \\
\text { Parlement }\end{array}$ & Remarques \\
\hline $\begin{array}{c}\text { Nombre de } \\
\text { rapports soumis }\end{array}$ & 157 & 159 & 1 & 14 & 1 & 0 & \\
\hline $\begin{array}{c}\text { Nombre de } \\
\text { rapports discutés } \\
\text { par le Parlement }\end{array}$ & & & 1 & & & & $\begin{array}{c}\text { Discutés par la } \\
\text { commission spécialisée } \\
\text { (programme de } \\
\text { contrôle de 2014) }\end{array}$ \\
\hline $\begin{array}{l}\text { Soumis au } \\
\text { Président }\end{array}$ & & & & & & & $\begin{array}{c}\text { Les rapports sont } \\
\text { soumis à l'Assemblée } \\
\text { nationale }\end{array}$ \\
\hline $\begin{array}{l}\text { Soumis au } \\
\text { Président de la } \\
\text { commission } \\
\text { spécialisée }\end{array}$ & & & & & & & \\
\hline $\begin{array}{c}\text { Copies envoyées } \\
\text { aux autres } \\
\text { commissions } \\
\text { concernées }\end{array}$ & & & & & & & $\begin{array}{c}\text { Oui, si cela est jugé } \\
\text { utile }\end{array}$ \\
\hline $\begin{array}{c}\text { Notes de synthèse } \\
\text { destinées à la } \\
\text { commission } \\
\text { parlementaire }\end{array}$ & & & & & & & \begin{tabular}{|c|} 
Les réunions entre les \\
membres des sous- \\
commissions et les \\
responsables de tous \\
les secteurs contrôlés \\
ont lieu pour discuter \\
des mesures à prendre \\
pour contrôler la mise \\
en œuvre des \\
recommandations \\
formulées par l'ISC
\end{tabular} \\
\hline $\begin{array}{c}\text { Résumés soumis } \\
\text { au Parlement }\end{array}$ & & & & & & & $\begin{array}{c}\text { En 2014, l'ISC a doté } \\
\text { les rapports d'audit } \\
\text { d'une nouvelle } \\
\text { structure. Voir la } \\
\text { section } 4\end{array}$ \\
\hline
\end{tabular}

3) Procédures parlementaires utilisées dans le traitement des rapports de l'ISC

Les Règles de procédure de l'Assemblée nationale prévoient en ce qui concerne I'ISC les dispositions suivantes : 
Article 55 :

La Commission des finances, du budget de l'État et du contrôle des dépenses publiques examine les projets de loi et les propositions concernant d'autres actes généraux et problématiques dans les domaines suivants :

- Système publique de financement, taxes, droits de douane et autres recettes publiques ;

- Budget de l'État et plans financiers des agences d'assurance sociale obligatoire ;

- États financiers du budget, états financiers finaux de plans de financement des agences d'assurance sociale obligatoire et contrôle des états financiers finaux ;

- Prêts, nantissements et loterie ;

- Dette publique et actifs financiers de la République de Serbie ;

- Marchés publics ;

- Systèmes de crédit, monétaire, banquier, des devises et des douanes ;

- Assurance d'actifs et de personnes ;

- Relations de propriété et expropriation ;

- Paiement et opérations de paiement, titres et marché monétaire ;

- Prévention du blanchiment d'argent et lutte contre la corruption ;

- Comptabilité et vérification des comptes ;

- Autres sujets du domaine financier.

La Commission :

- Examine les rapports de la Cour des comptes et soumet à l'Assemblée nationale un rapport contenant ses avis et recommandations ;

- Contrôle la mise en œuvre du budget de l'État et des plans financiers connexes au regard de la légalité, de l'adéquation et de l'efficience des dépenses publiques, et soumet à l'Assemblée nationale un rapport comprenant des recommandations sur les mesures à prendre.

La Commission exécute d'autres tâches en conformité avec les dispositions prévues par la Loi et avec les Règles de procédure.

La Commission comprend 17 membres.

Article 177:

La commission compétente examine la proposition d'états financiers finaux ainsi que le rapport de l'ISC sur son audit des états financiers de la République de Serbie, les états financiers des plans financiers des agences chargées de l'assurance sociale obligatoire et les rapports financiers consolidés de la République de Serbie, et soumet à l'Assemblé nationale un rapport en conformité avec les dispositions prévues par la Loi régissant I'ISC. 
Article 203 :

Des propositions destinées à l'élection et à la révocation du gouverneur de la Banque nationale de Serbie, au Conseil des gouverneur de la Banque nationale de Serbie, au défenseur des droits, au Commissaire à l'information d'importance publique et au Président, vice-président et membres du Conseil de la Cour des comptes, ainsi qu'à d'autres responsables déterminés par la loi, sont soumises accompagnées d'une argumentation à l'Assemblée nationale par les soumissionnaires autorisés.

Si un soumissionnaire autorisé pour l'élection d'un responsable visé à l'alinéa 1 du présent Article, élu par l'Assemblée nationale, n'est pas mentionné dans la loi, la proposition est soumise par la commission compétente de l'Assemblée nationale.

Avant l'élection des responsables visés à l'alinéa 1 du présent Article, nommés par la commission compétente de l'Assemblée nationale, des entretiens sont organisés avec les candidats, devant la commission compétente de l'Assemblée nationale.

Comme la Commission des finances, du budget de l'État et du contrôle des dépenses publiques, la souscommission pour l'examen des rapports des audits réalisés par la Cour de comptes a été constituée en février 2015. Les membres de la nouvelle sous-commission ont visité pour la première fois l'ISC et ont mis sur pied une coopération plus étroite afin que le contrôle des dépenses des fonds publics soit plus efficient. II a été convenu que I'ISC et la sous-commission établissent conjointement un système de surveillance des actions prises suite aux recommandations de I'ISC et de l'Assemblée nationale, dans le but d'améliorer le système financier et comptable de la République de Serbie. L'ISC est prête à aider la Commission des finances, du budget de l'État et du contrôle des dépenses publiques et la nouvelle souscommission à comprendre l'importance des constatations d'audit et à trouver les correctifs appropriés, sans compromettre son indépendance.

Dans les législatures de l'Assemblée nationale (au 14 février 2007 et 11 juin 2008), les présidents de la Commission des finances appartenaient aux partis d'opposition, tandis que dans les législatures de l'Assemblée nationale (au 31 mai 2012 et dans la convocation actuelle), les présidents de la Commission des finances appartenaient aux partis du gouvernement.

La commission ne nomme pas de rapporteur.

Il n'y a pas d'auditions auxquelles serait conviée l'entité contrôlée ou d'autres personnes concernées afin d'apporter des clarifications ou un complément d'informations.

Entre 2011 et 2015, les débats sur les rapports d'audit de l'ISC se déroulaient en commission. Les débats sur les rapports d'activité annuels se déroulaient en commission une fois par an. Les débats sur les rapports d'activité annuels ont eu lieu en plénière, sauf en 2015. La Commission des finances, du budget de l'État et des dépenses publiques de la République de Serbie, lors de la séance tenue le 18 mai 2015, a discuté du rapport d'activité annuel de 2014 et des propositions de conclusion (destinées aux délibérations en plénière) ont été élaborées lors des séances ultérieures.

Les rapports d'audit de l'ISC ont été examinés au sein de la Commission de contrôle des services de sécurité (rapport d'audit sur l'Agence sur les informations de sécurité). 
En vertu des Règles de Procédure de l'Assemblée nationale, la commission compétente examine les rapports de I'ISC puis soumet à I'Assemblée nationale un rapport contenant ses avis et ses recommandations.

Les Règles de Procédure de l'Assemblée nationale (Articles 171-178) définissent la procédure d'adoption du budget de la République de Serbie des états financiers finaux. La Loi relative aux comptes finaux du budget de la République de Serbie n'a pas été adoptée depuis 2002.

La commission dédiée à I'ISC examine la proposition faite sur les états financiers finaux ainsi que le rapport de I'ISC sur l'audit des états financiers de la République de Serbie, des états financiers des plans financiers des agences chargées de l'assurance sociale obligatoire et des rapports financiers consolidés de la République de Serbie ; elle soumet ensuite un rapport à l'Assemblée nationale conformément aux dispositions de la loi régissant I'ISC.

Le périmètre de contrôle de I'ISC est défini au titre de l'Article 92 de la BSL, sous réserve des dispositions détaillées énoncées dans la Loi relative à I'ISC, et l'obligation de joindre un rapport d'audit aux états financiers est stipulée à l'Article 79. Toutefois, la BSL ne fait pas état de délais quant à la soumission des états financiers à I'ISC, même si elle prescrit à l'Article 78 le délai (15 juillet) de soumission de ces états financiers (avec le rapport d'audit) à l'Assemblée nationale. Si ce délai n'est pas précisé, I'ISC considère que le temps qui lui est accordé pour contrôler les états financiers du budget de la République, au moment où le budget est soumis au Gouvernement (20 juin), est inadéquat.

En règle générale, les rapports d'audit identifient des problèmes de non-conformité à la BSL, ainsi que des emplois abusifs ou frauduleux des fonds. Tandis que ces emplois abusifs ou frauduleux sont traités par les tribunaux, les cas de non-conformité sont souvent systémiques et exigent que le Gouvernement prenne des actions correctives. Tandis que les problématiques les plus évidentes sont traitées en cours d'audit ou avant l'achèvement du rapport d'audit, il y a souvent une série de problèmes non réglés qui demandent un effort plus soutenu de la part du gouvernement. Ces problèmes devraient être relevés par l'Assemblée nationale lors de son examen des états financiers et du rapport d'audit, ce qui devrait amener une réponse écrite officielle du gouvernement sur les actions et les calendriers qu'il a prévus pour remédier à ces défaillances. Ce processus officiel devrait être défini dans la BSL. Il est nécessaire d'avoir des clarifications sur le calendrier destiné au traitement des états financiers, ainsi que sur l'organisation des laudits.

Pour que l'ISC respecte l'exigence selon laquelle les comptes finaux doivent être accompagnés du rapport d'audit, le délai relatif à la soumission officielle des états financiers à l'ISC devrait être le 30 avril, tandis que le délai de soumission à l'Assemblée nationale du rapport d'audit accompagnant les états financiers devrait être le 30 septembre. Un Article devrait être ajouté après l'Article 92 pour régir le processus et le calendrier de l'examen du compte final et du rapport final par l'Assemblée nationale, y compris l'obligation imposée au Gouvernement de donner suite de manière satisfaisante aux recommandations en attente.

\section{Personnel}

La Commission et la sous-commission ont deux assistants parlementaires. 


\section{Participation de l'ISC à l'examen de ses rapports par le Parlement}

L'ISC assiste aux réunions parlementaires lorsque ses rapports sont débattus. L'Auditeur général et les contrôleurs de haut rang, accompagnés du Secrétaire et du Chef de bureau du Président, assistent généralement à ces réunions. Les représentants de l'ISC présentent leurs constatations et répondent aux questions des parlementaires.

\section{4) Rapports de I'ISC}

En 2014, I'ISC a modifié la structure des rapports d'audit. Un document de base est préparé pour chaque audit réalisé. Ce document contient l'opinion de l'Auditeur général sur les états financiers et l'audit de conformité. Avant la section des opinions, des sous-sections couvrent la responsabilité des organes dirigeants dans la préparation des états financiers et en matière de conformité des opérations, ainsi que le fondement de l'opinion, incluant les irrégularités relevées.

L'Annexe 1 contient les irrégularités essentielles révélées par le processus d'audit, notamment par l'audit financier et l'audit de conformité. Elle formule également les recommandations visant à supprimer les irrégularités ainsi que les mesures d'amélioration mises en place par la Direction de l'entité contrôlée.

L'Annexe 2 comprend les notes sur le rapport d'audit. Cette partie explique de manière détaillée toutes les irrégularités détectées.

L'Annexe 3 regroupe les états financiers de l'entité contrôlée.

Chaque rapport d'audit de performance contient une page récapitulative indiquant le titre du rapport, les raisons de l'audit, les constatations et les recommandations de I'ISC.

Les rapports d'audit de I'ISC présentent des informations détaillées sur le statut des systèmes de contrôle internes, ainsi que des conclusions et des opinions claires.

\section{5) Suivi des rapports de l'ISC}

L'ISC assure le suivi de ses recommandations dans sa base de données interne. Il est prévu de créer une base de données publique contenant les recommandations d'audit, pour faciliter l'identification des problèmes systémiques et renforcer le socle des informations destinées au contrôle parlementaire sur l'exécutif.

Le rapport d'activité annuel de I'ISC présente en résumé les opinions de l'ISC, les irrégularités constatées, les conséquences de l'audit, et les recommandations faites aux entités contrôlées. À une occasion, suite à la conclusion de I'Assemblée nationale sur le rapport d'activité de 2012, I'ISC a soumis à l'Assemblée nationale un rapport indépendant portant sur la mise en œuvre des recommandations énoncées dans les rapports d'audit de 2011. Ce rapport comprenait une vue générale des activités contrôlées par l'ISC en 2013.

L'efficacité des travaux de I'ISC est pénalisée par l'absence de procédures et de délais pour l'examen des rapports d'audit par la Commission des finances, du budget de l'État et du contrôle des dépenses publiques. À ce jour, il n'a été tenu aucune audition sur les constatations documentées dans les rapports de I'ISC pour obliger les représentants de l'exécutif à rendre compte. La sous-commission chargée de l'examen des rapports d'audit établis par l'ISC a été créée en février 2015. L'ISC a informé les membres de 
cette sous-commission des faiblesses systémiques relevées dans les audits, pour que cette dernière les examinent pendant la session suivante et prennent les mesures appropriées.

Le gouvernement dans son ensemble n'est pas obligé d'établir un rapport sur la mise en œuvre des recommandations de I'ISC. Chaque organisme contrôlé doit soumettre une réponse à I'ISC expliquant comment il a mis en œuvre ses recommandations.

En matière de soumission des comptes finaux contrôlés (décembre), la méthode actuellement appliquée limite l'utilité des informations fournies dans le rapport d'audit lors de la délibération de la proposition de budget par le Parlement. II est nécessaire de faire respecter l'obligation constitutionnelle et réglementaire imposée à l'Assemblée nationale dans l'examen et l'adoption des comptes finaux du budget de l'État, cette obligation constituant un maillon crucial dans la chaîne des responsabilités. Sans contrôle ex-post du budget, l'Assemblée ne peut pas obliger l'exécutif à rendre compte de l'utilisation des ressources allouées et proposer des améliorations sur leur gestion. La pratique actuelle limite la capacité de l'Assemblée nationale à examiner la fiabilité, l'exactitude, l'exhaustivité et la conformité aux règles des comptes finaux. Elle empêche en outre d'avoir un débat sérieux pour savoir dans quelle mesure le budget a été utilisé aux fins énoncées dans le budget approuvé.

\section{6) Audits réalisés à la demande du Parlement}

II n'y a pas de disposition juridique qui permettrait (ou empêcherait) le Parlement de solliciter des audits.

À une occasion, à la séance de la Commission de contrôle des services de sécurité, le Président de la Commission a proposé que I'ISC réalise un audit financier de l'Agence sur les informations de sécurité, et cet audit a été mené l'année suivante.

\section{7) Programme de travail}

Pendant l'élaboration du plan stratégique 2016 - 2020, I'ISC a interrogé les parlementaires siégeant à la Commission et sous-commission sur ce qu'ils attendent de I'ISC durant la période stratégique suivante. L'ISC a accordé une grande importance aux informations recueillies.

\section{8) Niveau et fréquence des échanges}

II y a régulièrement des échanges (une fois par mois) entre le président de I'ISC et le président de la commission parlementaire compétente. Dans le cadre du travail, des échanges ont lieu au quotidien entre les secrétaires de la Commission et sous-commission et le Chef du Bureau du Président.

\section{9) Bonnes pratiques}

L'ISC a organisé plusieurs ateliers, tables rondes, conférences et visites d'étude dans le but de renforcer ses relations avec le Parlement.

Au titre du projet jumelé intitulé "Renforcement des capacités de la Cour des comptes de la République de Serbie ", il a été élaboré des lignes directrices portant sur les relations avec le Parlement, les entités contrôlées et les médias.

\section{0) Perspective}

Dans le cadre du programme de réforme de la gestion des finances publiques 2016 - 2020, il a été prévu d'améliorer le suivi systématique de la mise en œuvre des recommandations d'audit. Ces mesures comprennent la création d'une base de données dédiée aux recommandations, ce qui facilitera la 
découverte des problèmes systémiques et l'amélioration du socle des informations permettant le contrôle de l'exécutif par les parlementaires. Elles fourniront de surcroit au Parlement l'assurance de pouvoir exercer une fonction de contrôle adéquate. Pour cela, il faudra mettre en place les procédures organisationnelles destinées aux travaux de contrôle externes et mettre par écrit des procédures claires encadrant la discussion des rapports d'audit, avec les délais relatifs aux discussions et aux actions de la Commission des finances, du budget de l'État et du contrôle des dépenses publiques. Ce projet couvrira également l'organisation des réunions de cette Commission pour débattre des rapports d'audit.

11) Y-a-t-il d'autres éléments concernant les relations actuelles entre l'ISC et le Parlement que vous souhaiteriez souligner, et quelles a été leur évolution au cours des cinq dernières années?

Le Président de la Cour des comptes, le Vice-président de l'Assemblée nationale et le Président de la Commission des finances, du budget de l'État et du contrôle des dépenses publiques ont signé au nom de la Commission et de I'ISC un protocole d'accord le 10 juin 2015. 


\section{RÉPUBLIQUE SLOVAQUE}

Bureau de contrôle supérieur

\section{1) Cadre juridique}

\section{Constitution de la République Slovaque :}

\section{Article 62:}

Le Bureau de contrôle supérieur soumet au Conseil national de la République slovaque des rapports sur les résultats de ses travaux d'audit au moins une fois par an et chaque fois que le Conseil national de la République slovaque le demande.

\section{Loi sur le BCS RS :}

\section{Article 5 :}

(1) Le Bureau formule une opinion sur la proposition du budget de l'État de la République slovaque, par laquelle il évalue en outre la proposition relative au budget de l'administration de l'État, et une opinion sur la proposition du compte final de l'État de la République slovaque. Le Bureau soumet au Conseil national de la République slovaque son opinion sur la proposition du budget de l'État de la République slovaque dans les délais définis par un règlement spécial. 1e) Le Bureau soumet au Conseil national de la République slovaque son opinion sur la proposition du budget de l'État de la République dans les 30 jours suivant la décision du Gouvernement.

(2) Le Bureau exécute les tâches d'un organe qui émet des déclarations sur la clôture de l'assistance financière octroyée au titre de fonds structurels $1 \mathrm{f}$ ) les tâches d'un organe qui émet des déclarations sur la liquidation de l'assistance financière octroyée au titres du fonds de cohésion $1 \mathrm{~g}$ ) ; il est responsable du contenu desdites déclarations et il les soumet aux autorités compétentes.

(3) Le Bureau exécute les tâches d'un organe certificateur pour le fonds européen d'orientation et de garantie agricole- Section Garantie. 1h).

(4) En s'appuyant sur la résolution du Conseil national de la République slovaque, le Bureau exerce un audit dans le périmètre de ses compétences, pour le compte du Conseil national de la République slovaque.

(5) Au plus tard à la fin du mois de mars de l'année suivante, le Bureau soumet au Conseil national de la République slovaque un résumé du rapport sur les résultats des travaux de contrôle pour l'année calendaire précédente. Le rapport sur les résultats des travaux de contrôle est soumis chaque fois que le Conseil national de la République slovaque le demande.

\section{Article 8:}

(1) Le Bureau est dirigé par le Président en qualité d'organe institutionnel. Le Président est assisté dans ses fonctions par le Vice-président désigné.

(2) Le Président et deux Vice-présidents sont élus et révoqués à bulletin secret par le Conseil national de la République slovaque. 
(3) Le mandat du Président et des deux Vice-présidents est de sept ans.

(4) Le Président et les deux Vice-présidents peuvent être élus à leur charge pour deux mandats consécutifs maximum.

(5) Le Président assure ses fonctions après la fin de son mandat jusqu'à ce qu'un nouveau Président soit élu par le Conseil national de la République slovaque.

Article 12 :

(1) La charge du Président et des Vice-présidents est terminée par :

a) expiration du mandat officiel, à moins que la présente loi n'en dispose autrement ;

b) résiliation;

c) révocation de ses fonctions.

(2) Le Président ou les Vice-présidents sont révoqués de leurs fonctions par le Conseil national de la République slovaque quand:

a) il a été condamné par la loi d'une infraction intentionnelle ;

b) il a occupé un poste ou exécuté une activité incompatible avec les fonctions de président ou vice-présidents en vertu de l'Article 10 ;

c) il n'exécute pas les obligations visées par la présente Loi.

(3) Le Conseil national de la République slovaque peut révoquer le Président ou Vice-président s'il n'exécute pas les obligations liées à ses fonctions pendant une période dépassant six mois consécutifs.

Article 13:

(1) Le Président informe le Conseil national de la République slovaque et tout autre organe concerné des constatations et des informations importantes relevant du domaine de sa charge.

Article 14 :

Le Président a le droit d'être présent aux séances du Conseil national de la République slovaque et de ses organes.

Article 20:

Au cas où le Gouvernement manquerait à ses obligations en vertu de l'alinéa 2, le Président consulte le Premier ministre sur l'affaire. Si aucun accord ne peut être obtenu, le Président dépose l'affaire devant le Conseil national de la République slovaque.

Article 21a:

(1) Le budget du Bureau est l'objet d'un chapitre individuel dans le budget de l'État de la République slovaque. 
(2) Le Bureau soumet une version provisoire du chapitre du budget pour approbation par la commission compétente du Conseil national de la République slovaque. Ladite commission soumet la version provisoire approuvée du chapitre du budget au Bureau du Gouvernement d'ici le 30 juin de l'année en cours. Si le Gouvernement apporte des modifications à la version provisoire du chapitre du budget du Bureau, il motivera ces modifications lorsque le projet de loi relatif au budget de l'État sera soumis au Conseil national de la République slovaque.

(3) Le Bureau est responsable auprès du Conseil national de la République slovaque de sa gestion des fonds du budget de l'État, en vertu du chapitre sur le budget du Bureau.

(4) Le Conseil national de la République slovaque contrôle la gestion des actifs de l'État, y compris des fonds du budget de l'État, en vertu du chapitre sur le budget du Bureau, par le Bureau.

\section{2) Modalités de soumission des rapports de l'ISC au parlement}

L'Institution supérieure d'audit (SAO) soumet séparément aux commissions compétentes ses rapports relatifs aux différents audits. Les autres types de rapports déposés devant le Conseil national de la République slovaque sont :

- Un rapport résumant les résultats des travaux d'audit pour l'année calendaire antérieure au plus tard à la fin du mois de mars ;

- Une opinion sur la proposition du budget de l'État de la République slovaque dans les délais fixés par un règlement spécial ;

- Une opinion sur la proposition du compte final de l'État de la République slovaque au Conseil national dans les 30 jours suivant la décision du Gouvernement.

\section{Procédures d'envoi des rapports au Parlement}

L'envoi des rapports au Parlement n'est pas régi par des procédures spécifiques. Les opinions sur la proposition du budget de l'État de la République et sur la proposition du compte final de l'État sont présentées par le Président de l'ISC à la Commission budgétaire au Parlement réuni en séance plénière. 
Nombre et type de rapports

\begin{tabular}{|c|c|c|c|c|c|c|c|}
\hline & $\begin{array}{l}\text { Rapports } \\
\text { d'audit } \\
\text { financier }\end{array}$ & $\begin{array}{c}\text { Rapports d'audit } \\
\text { de conformité }\end{array}$ & $\begin{array}{l}\text { Rapports d'audit } \\
\text { de performance }\end{array}$ & $\begin{array}{c}\text { Autres rapports } \\
\text { ou documents }\end{array}$ & $\begin{array}{l}\text { Rapports } \\
\text { d'activité } \\
\text { annuels }\end{array}$ & $\begin{array}{c}\text { Rapports } \\
\text { demandés par } \\
\text { le Parlement }\end{array}$ & Remarques \\
\hline $\begin{array}{c}\text { Nombre de } \\
\text { rapports soumis }\end{array}$ & \multicolumn{3}{|c|}{30} & 2 & 1 & & \\
\hline $\begin{array}{c}\text { Nombre de } \\
\text { rapports discutés } \\
\text { par le Parlement }\end{array}$ & & & & 2 & 1 & & \\
\hline \multicolumn{8}{|l|}{$\begin{array}{l}\text { Soumis au } \\
\text { Président }\end{array}$} \\
\hline \multicolumn{8}{|l|}{$\begin{array}{l}\text { Soumis au } \\
\text { Président de la } \\
\text { commission } \\
\text { spécialisée }\end{array}$} \\
\hline $\begin{array}{l}\text { Copies envoyées } \\
\text { aux autres } \\
\text { commissions } \\
\text { concernées }\end{array}$ & \multicolumn{3}{|c|}{30} & & & & \\
\hline \multicolumn{8}{|l|}{$\begin{array}{l}\text { Notes de synthèse } \\
\text { destinées à la } \\
\text { commission } \\
\text { parlementaire }\end{array}$} \\
\hline $\begin{array}{c}\text { Résumés soumis au } \\
\text { Parlement }\end{array}$ & & & & & & & \\
\hline
\end{tabular}

3) Procédures parlementaires utilisées dans le traitement des rapports de l'ISC

En ce qui concerne le traitement des rapports de I'ISC, des procédures établies sont inscrites dans les règles de procédure du Parlement. Toutefois, ces procédures sont identiques à celles de n'importe quels autres rapports soumis au Parlement. II n'existe pas de commission dédiée au traitement des rapports de I'ISC. L'ISC soumet généralement tous les rapports concernant des audits individuels à la commission parlementaire compétente, selon le sujet de l'audit.

Il y a rarement des auditions auxquelles est conviée l'entité contrôlée ou d'autres personnes concernées afin d'apporter des clarifications ou un complément d'informations, et ce uniquement dans des cas très importants. II ne s'agit pas d'une procédure courante.

Le Parlement vote une résolution contenant une conclusion qui dépend du sujet traité. 


\section{Personnel}

II n'y a pas de commission parlementaire spécifiquement chargée du traitement des rapports d'audit. Chaque commission comprend une douzaine de membres. Le personnel parlementaire fournit uniquement une assistance administrative à la (aux) commission(s) mais les différents parlementaires ont des conseillers externes pour leur apporter assistance et support.

\section{Participation de l'ISC à l'examen de ses rapports par le Parlement}

L'ISC assiste toujours aux réunions parlementaires de la Commission budgétaire pour présenter une opinion sur la proposition relative au budget de l'État et une opinion sur la proposition du compte final de l'État. Dans ce domaine, la Commission budgétaire du Parlement est la commission compétente. L'ISC est généralement représentée par son Vice-président et le Directeur général responsable de l'opinion.

\section{4) Rapports de I'ISC}

Dans sa communication avec le Parlement, I'ISC se concentre sur des problèmes génériques / systémiques et sur la formulation de recommandations.

La configuration et le fonctionnement des systèmes de contrôle internes sont des sujets mis en avant dans les rapports de I'ISC.

Tous les rapports d'audit contiennent des conclusions et des opinions claires.

\section{5) Suivi des rapports de l'ISC}

La Loi relative à I'ISC détermine à l'intention des entités contrôlées l'obligation de soumettre à l'ISC les mesures correctives et à rendre compte de l'application de ces mesures pour chaque audit dans les délais convenus, documentés dans les minutes de clôture des audits.

Le principe de suivi adopté par I'ISC consiste à examiner sur place les mesures correctives et les recommandations formulées dans l'audit, le cas échéant. Des audits indépendants se concentrent spécialement sur le suivi et le pilotage des mesures correctives et des recommandations sont transmises par le biais de systèmes d'informations spécialisés.

\section{6) Audits réalisés à la demande du Parlement}

Sur la base d'une résolution prise par le Conseil national de la République slovaque, I'ISC a la faculté de réaliser un audit dans le périmètre de ses compétences à la demande du Conseil national de la République slovaque. Ce cas est très rare ; il est arrivé peut-être une ou deux fois en deux ou trois ans.

\section{7) Programme de travail}

L'ISC ne consulte pas le Parlement sur son plan de contrôle annuel. Pendant le processus de planification annuel, elle évalue très soigneusement l'ensemble des suggestions et des propositions publiques disponibles. Les suggestions et les propositions provenant du Parlement ne sont pas juridiquement contraignantes, mais I'ISC les évalue néanmoins. 


\section{8) Niveau et fréquence des échanges}

II n'y a pas d'échanges réguliers entre le président de I'ISC et les présidents des commissions parlementaires. Les échanges officiels découlent des responsabilités réglementaires de I'ISC qui l'obligent à soumettre des rapports. II n'y a pas de personnel ou d'unité de I'ISC spécifiquement affecté aux relations parlementaires.

\section{9) Bonnes pratiques}

L'ISC ne souhaite rien mettre en avant.

\section{0) Perspective}

La priorité de I'ISC pour l'avenir est d'intensifier ses contacts avec les commissions compétentes du Parlement slovaque, le Conseil national de la République slovaque. Outre les réunions officielles, I'ISC souhaite introduire des réunions informelles dans le but de discuter des thématiques qui préoccupent les deux parties.

11) Y-a-t-il d'autres éléments concernant les relations actuelles entre l'ISC et le Parlement que vous souhaiteriez souligner, et quelles a été leur évolution au cours des cinq dernières années?

Depuis cinq ans, les relations entre l'ISC et le Parlement sont bonnes. L'ISC a exécuté deux ou trois audits à la demande du Parlement. 


\section{SLOVÉNIE}

Cour des comptes

\section{1) Cadre juridique}

La Constitution de la République de Slovénie compte deux articles sur la Cour des comptes (CoA), les Articles 150 et 151 . Ils définissent la CoA comme étant une institution indépendante dans l'accomplissement de ses devoirs et liée par la Constitution et par la loi (Alinéa 3 Article 150), qui stipule que les membres de la CoA sont nommés par l'Assemblée nationale (Article 151).

Les obligations de la CoA envers le Parlement (Moniteur officiel de la République de Slovénie, №11/01 et 109/12) stipulent ce qui suit :

- Au moins une fois par an, la Cour des comptes soumet à l'examen de l'Assemblée nationale un rapport sur ses travaux (alinéa 2 Article 5 )

- Le rapport visé à l'Article 5, alinéa 2, de la présente Loi doit contenir : la liste de toutes les propositions d'audit faites par les députés et les organes de travail de l'Assemblée nationale ;

- Des justificatifs probants démontrant que les audits ont pris en compte ces propositions. (alinéa 3, Article 25).

- Lorsqu'elle détermine les audits à exécuter pendant une année calendaire donnée, la Cour des comptes examine les propositions faites par les députés et les instances de travail de l'Assemblée nationale, du Gouvernement, des ministres et des collectivités locales. Elle doit examiner au moins cinq propositions provenant de l'Assemblée nationale, parmi lesquelles deux doivent être soumises par des députés de l'opposition et deux autres par des instances de travail de l'Assemblée nationale. (alinéa 2, Article 25).

- Une fois la procédure de contrôle terminée, le rapport d'audit est remis :

○ À l'entité contrôlée ;

- Au responsable de l'entité contrôlée au cours de la période examinée par l'audit ;

○ À l'Assemblée nationale ;

- À d'autres autorités qui, de l'avis du Président de la Cour des comptes, devraient être informées des conclusions de l'audit. (alinéa 16, Article 28).

- Dans la procédure postérieure à l'audit, si l'obligation d'efficience opérationnelle a été gravement violée, la cour des comptes le notifie à l'Assemblée nationale. (alinéa 8, Article 29).

\section{2) Modalités de soumission des rapports de l'ISC au parlement}

La Cour des comptes est tenue par la loi de transmettre les rapports d'audit à l'Assemblée nationale dès leur achèvement. Cette règle vaut pour tous les types de rapports (financiers, de conformité, de performance, des rapports postérieurs aux audits). L'Assemblée nationale reçoit le rapport d'audit sous sa forme originale, sans supplément ou résumé à son intention.

Les rapports sont adressés à l'Assemblée nationale en général, et non à une personne ou un organe en particulier. Comme tous les rapports doivent être soumis une fois terminés à l'Assemblée nationale, ils lui sont envoyés au même moment qu'aux entités contrôlées, c'est-à-dire immédiatement. 
La Cour des comptes envoie son rapport annuel une fois par an à l'Assemblée nationale. II est généralement publié fin février ; aucun délai particulier n'est fixé pour cet envoi. Il est habituellement remis personnellement par le Président de la CoA au Président de l'Assemblée nationale.

\section{Procédures d'envoi des rapports au Parlement}

Il n'y a pas de procédures particulières en vigueur. Une copie du rapport d'audit est envoyée simultanément à l'Assemblée nationale et à l'entité contrôlée.

Le CoA ne réalise pas d'autres travaux, sauf si elle est invitée à le faire par l'Assemblée nationale ou par ses instances de travail. 
Nombre et type de rapports

\begin{tabular}{|c|c|c|c|c|c|c|c|}
\hline & $\begin{array}{l}\text { Rapports } \\
\text { d'audit } \\
\text { financier }\end{array}$ & \begin{tabular}{|c|} 
Rapports d'audit \\
de conformité
\end{tabular} & $\begin{array}{l}\text { Rapports d'audit } \\
\text { de performance }\end{array}$ & $\begin{array}{l}\text { Autres rapports } \\
\text { ou documents }\end{array}$ & $\begin{array}{c}\text { Rapports } \\
\text { d'activité } \\
\text { annuels }\end{array}$ & $\begin{array}{c}\text { Rapports } \\
\text { demandés par } \\
\text { le Parlement }\end{array}$ & Remarques \\
\hline $\begin{array}{c}\text { Nombre de } \\
\text { rapports soumis }\end{array}$ & $30(45 \%)$ & $16(25 \%)$ & $18(28 \%)$ & $\begin{array}{l}1 \text { (rapport } \\
\text { résumé) }\end{array}$ & 1 & Sans objet & \\
\hline $\begin{array}{c}\text { Nombre de } \\
\text { rapports discutés } \\
\text { par le Parlement }\end{array}$ & 3 & 5 & 12 & 0 & Sans objet & Sans objet & \\
\hline $\begin{array}{l}\text { Soumis au } \\
\text { Président }\end{array}$ & Sans objet & Sans objet & Sans objet & Sans objet & Sans objet & Sans objet & \\
\hline $\begin{array}{l}\text { Soumis au } \\
\text { Président de la } \\
\text { commission } \\
\text { spécialisée }\end{array}$ & Sans objet & Sans objet & Sans objet & Sans objet & Sans objet & Sans objet & \\
\hline $\begin{array}{c}\text { Copies envoyées } \\
\text { aux autres } \\
\text { commissions } \\
\text { concernées }\end{array}$ & Sans objet & Sans objet & Sans objet & Sans objet & Sans objet & Sans objet & \\
\hline $\begin{array}{l}\text { Notes de synthèse } \\
\text { destinées à la } \\
\text { commission } \\
\text { parlementaire }\end{array}$ & Sans objet & Sans objet & Sans objet & Sans objet & Sans objet & Sans objet & $\begin{array}{c}\text { La CoA } \\
\text { assiste aux } \\
\text { présentations } \\
\text { d'information } \\
\text { si elles sont } \\
\text { organisées } \\
\text { par } \\
\text { l'Assemblée } \\
\text { nationale }\end{array}$ \\
\hline $\begin{array}{c}\text { Résumés soumis au } \\
\text { Parlement }\end{array}$ & Sans objet & Sans objet & Sans objet & Sans objet & Sans objet & Sans objet & $\begin{array}{c}\text { Le résumé du } \\
\text { rapport } \\
\text { d'audit est } \\
\text { intégré au } \\
\text { rapport } \\
\text { publié }\end{array}$ \\
\hline
\end{tabular}

\section{3) Procédures parlementaires utilisées dans le traitement des rapports de l'ISC}

Une fois terminé, le rapport d'audit est publié et envoyé simultanément à l'entité contrôlée et à I'Assemblée nationale. II n'existe pas de procédures spéciales pour examiner les rapports de I'ISC. Toutefois, après avoir été reçu par l'Assemblée nationale, les rapports sont transmis à la Commission de 
contrôle des finances publiques (CPFC), qui est une commission permanente composée de neuf membres et présidée par un membre de l'opposition. Son mandat, ses fonctions et sa composition sont définis par les règles de procédure de l'Assemblée nationale (Article 39).

La CPFC tient une audition, à laquelle elle peut convier la CoA et les entités contrôlées lorsqu'un rapport d'audit est discuté, afin d'apporter des clarifications ou un complément d'informations. La Commission peut en outre faire appel à des experts externes si elle juge cela nécessaire. Toutefois, ces examens réalisés par des experts ne sont pas et ne peuvent pas servir à vérifier les rapports d'audit de la CoA.

Le débat sur les rapports de I'ISC se déroule généralement au sein de la commission, mais il peut également avoir lieu en plénière, cette deuxième option étant considérée comme une action spéciale.

Les rapports d'audit de la CoA peuvent et sont examinés par d'autres commissions que la CPFC, par exemple par la Commission des affaires de l'Union européenne, la Commission de l'agriculture, la Commission des infrastructures, pour n'en citer que quelques-unes. Parfois, les rapports d'audit sont examinés puis la CoA est invitée à des séances ad hoc tenues par la commission chargée d'une thématique particulièrement importante pour l'Assemblée nationale.

La CPFC publie les minutes des différentes séances; ces minutes comprennent à la fois les conclusions et les demandes d'action. Ces demandes peuvent être adressées aux entités ou organes contrôlés, mais pas à la CoA.

En matière de décharge, le rôle de l'Assemblée nationale de la Slovénie consiste à adopter le rapport annuel sur l'exécution du budget. La CoA n'intervient pas dans la procédure de décharge.

\section{Personnel}

Aucun collaborateur parlementaire n'assiste la commission dans son traitement des rapports de l'ISC.

\section{Participation de l'ISC à l'examen de ses rapports par le Parlement}

La CoA ne participe pas à la préparation des auditions aux côtés de la commission.

La CoA assiste aux réunions parlementaires lorsque ses rapports sont débattus uniquement sur invitation de la Commission / I'Assemblée. Cette invitation est adressée au Président de la CoA qui assiste généralement aux réunions accompagné d'autres membres de la CoA, selon l'audit et la thématique débattus, à savoir le Vice-président (ou les deux vice-présidents), les chefs des divisions de contrôle (Auditeurs suprême de l'état), les responsables des audits ou les conseillers.

La contribution des représentants de la CoA est limitée aux audits exécutés. Uniquement lorsqu'une discussion générale est ouverte, les membres de la CoA (le Président, le premier et deuxième viceprésident) communiquent leurs observations fondées sur des constatations mentionnées également dans d'autres audits, portant sur la problématique ou la politique discutée. 


\section{4) Rapports de l'ISC}

La CoA se concentre sur des problèmes génériques/systémiques et sur la formulation de recommandations qui ne sont pas contraignantes par la loi pour les entités contrôlées. Elles constituent uniquement les vues de la CoA sur la question.

La configuration et le fonctionnement des systèmes de contrôle internes ne sont pas des sujets mis en avant dans les rapports de I'ISC. Toutefois, la CoA formule des recommandations sur les contrôles internes (notamment sur la détection des risques et des irrégularités).

En Slovénie, formuler clairement des conclusions et des opinions est un critère obligatoire pour tous les types d'audit. La Loi relative à la Cour des comptes stipule à l'Article 20 :

1. La Cour des comptes contrôle les activités des utilisateurs de fonds publics :

a) Elle est habilité à exécuter des audits de régularité et de performance ;

b) Elle peut contrôler tout acte portant sur des opérations passées ainsi que toute activité planifiée par un quelconque utilisateur de fonds publics.

2. Le contrôle des activités, conformément à la présente Loi, consiste à obtenir des données probantes et suffisantes pour exprimer une opinion sur ces activités :

a) Les audits de régularité fournissent des données probantes et suffisantes pour permettre d'exprimer une opinion sur la conformité des activités par rapport aux réglementations et aux lignes directrices que tout utilisateur de fonds publics est tenu d'observer dans la conduite de ses activités ;

Les audits de performance fournissent des données probantes et suffisantes pour permettre d'exprimer une opinion sur l'économie, l'efficience et l'efficacité de l'activité.

\section{5) Suivi des rapports de l'ISC}

La CoA conserve une trace des conclusions et des recommandations acceptées par l'entité contrôlée car en Slovénie, la CoA est habilitée à formuler non seulement des recommandations non contraignantes, mais également des demandes d'action, qui sont contraignantes pour l'entité contrôlée et leur mise en œuvre est vérifiée par la procédure postérieure à l'audit, par la réponse que l'entité contrôlée doit rédiger sur les mesures prises.

Puisque la procédure postérieure à l'audit a pour but de renforcer l'acceptation de l'entité contrôlée des demandes d'action, la CPFC fait attention à la réaction de l'entité contrôlée envers le rapport d'audit publié.

Le gouvernement ayant le même statut que n'importe quelle autre entité contrôlée, il est obligé de soumettre une réponse aux demandes d'action formulées dans le rapport d'audit.

La CoA n'a pas la faculté d'évaluer comment sont utilisées ses recommandations dans le débat annuel sur le projet de budget. 


\section{6) Audits réalisés à la demande du Parlement}

L'Assemblée nationale a le droit de proposer et de soumettre des suggestions d'audit, mais elle n'a pas la capacité de les demander ou de les exiger. La CoA élabore son plan de contrôle de manière totalement indépendante, mais elle doit prendre en compte les suggestions reçues et les intégrer dans son plan.

La Loi sur la Cour des comptes accorde à l'Assemblée nationale le droit de soumettre les propositions visées aux alinéas 2 et 3 de l'Article 25 :

(1) Dans le cadre fixé par la loi, la Cour des comptes sélectionne de manière autonome les audits qu'elle exécutera sur des périodes déterminées.

(2) Lorsqu'elle détermine les audits à exécuter pendant une année calendaire donnée, la Cour des comptes examine les propositions faites par les députés et les instances de travail de l'Assemblée nationale, du gouvernement, des ministres et des collectivités locales. Elle doit examiner au moins cinq propositions provenant de l'Assemblée nationale, parmi lesquelles deux doivent être soumises par des députés de l'opposition et deux autres par des instances de travail de l'Assemblée nationale.

(3) Le rapport visé à l'Article 5, alinéa (2) de la présente Loi doit contenir :

1. La liste de toutes les propositions d'audit faites par les députés et les instances de travail de I'Assemblée nationale ;

2. Des justificatifs démontrant clairement les propositions d'audit retenues.

En 2015, la CoA a reçu 10 propositions de l'Assemblée nationale : une de la plénière, 6 de la CPFC et 3 de différents députés ou groupes de députés. Elles ont été intégrées aux activités de la CoA, à différents stades des procédures de contrôle et dans certaines rubriques du plan annuel. La décision portant sur les modalités d'intégration des propositions dans le plan de contrôle annuel est exclusivement celle du Président de la CoA.

\section{7) Programme de travail}

Voir la section précédente.

\section{8) Niveau et fréquence des échanges}

II y a régulièrement des échanges entre le président de I'ISC et le président de la CPFC, principalement à la suite des travaux menés par la CPFC au regard des rapports d'audit publiés, mais également dans le cadre de discussions plus informelles sur les finances publiques et les problèmes de gouvernance.

\section{9) Bonnes pratiques}

Comme la CoA envoie tous ses rapports à l'Assemblée nationale au moment de leur publication, la CPFC est tenue informée de son travail et elle peut présenter les informations ou les thèmes et les préoccupations évoqués dans les rapports à ses séances ou en séance plénière du Parlement.

Un autre exemple de bonnes pratiques concerne la remise du rapport annuel de la CoA au Président du Parlement, lorsque le Président de I'ISC et le Président de l'Assemblée nationale discutent de l'influence des travaux de I'ISC, soulignent les sujets d'intérêt commun et déterminent les éventuelles évolutions dans la coopération. 


\section{0) Perspective}

La CoA estime qu'elle a de bonnes et régulières relations de travail avec l'Assemblée nationale, ce qui permet aux deux institutions de renforcer mutuellement des éléments de leur coopération, de résoudre de manière satisfaisante les problèmes de délai et d'exploiter leur potentiel afin de trouver une solution aux problèmes relatifs aux finances publiques et à la gouvernance, au bénéfice de la société slovène. 


\section{ESPAGNE}

Cour des comptes

\section{1) Cadre juridique}

Le Tribunal de Cuentas (ISC d'Espagne) rend compte directement au Parlement espagnol, avec qui il a un contact permanent par le biais de la Commission mixte du Congrès et du Sénat pour les relations avec le Tribunal de Cuentas.

Le Parlement espagnol a légalement le droit de solliciter auprès du Tribunal de Cuentas d'exécuter des audits spécifiques, par décision de la Commission mixte pour les relations avec le Tribunal de Cuentas. La Cour doit intégrer ces initiatives dans son programme de contrôle, en tenant compte de ses ressources disponibles. Voir également la section 136 de la Constitution espagnole.

La séance plénière du Tribunal de Cuentas approuve chaque année le programme de contrôle puis l'envoie à la Commission mixte pour les relations avec le Tribunal de Cuentas.

Le résultat des travaux d'audit figure dans les rapports, les mémorandums, les motions et les notes de travail approuvés en séance plénière du Tribunal de Cuentas et envoyés au Parlement.

Le Président du Tribunal de Cuentas présente régulièrement au Parlement les rapports approuvés et publiés par le Tribunal de Cuentas tout au long de l'année car ces rapports sont approuvés en plénière, lors des réunions régulières de la Cour. Un rapport annuel est également présenté à ces réunions.

Suite à l'audition du Président du Tribunal de Cuentas, la Commission mixte du Congrès et du Sénat chargée des relations avec le Tribunal de Cuentas délibère sur les différents rapports, mémorandums, motions et notes de service, et prend les décisions appropriées (pour plus d'informations, voir la Loi organique et la Loi sur le fonctionnement du Tribunal de Cuentas).

\section{2) Modalités de soumission des rapports de l'ISC au parlement}

Le Tribunal de Cuentas envoie au Parlement tous les rapports approuvés et publiés, à savoir les rapports d'audit financiers, de conformité, de performance, ou bien des mémorandums, des motions et des notes de service.

Le Tribunal de Cuentas établit un rapport par audit, mais également un rapport sur les comptes généraux de l'État, qui comprend les audits de plusieurs organismes.

Le Tribunal de Cuentas espagnol adopte le rapport annuel de ses activités avant de l'envoyer au Parlement. Les rapports sont adressés aux présidents du Congrès et du Sénat.

Le programme de contrôle annuel est adressé au Président de la Commission mixte.

L'Article 132 de la Loi générale relative au budget (examen et vérification des comptes généraux de l'État) stipule que, par délégation du Parlement, le Tribunal de Cuentas examine et vérifie le Compte général de l'État dans les six mois suivant sa réception. Suite à une audition du procureur, la Cour promulgue la déclaration définitive qu'elle juge opportune et envoie le compte et sa proposition au Parlement, en informant simultanément le gouvernement. 
Le Compte général de l'État est élaboré par le Contrôleur général de l'État, qui l'envoie ensuite au Tribunal de Cuentas avant le 31 octobre de l'année suivante.

Les autres rapports d'audit n'ont pas de délais établis, mais des délais internes sont fixés dans les directives techniques des audits spécifiques.

\section{Procédures d'envoi des rapports au Parlement}

L'envoi des rapports au Tribunal de Cuentas n'est régi par aucune procédure spécifique.

Son Président remet tous les rapports à la commission parlementaire. L'année dernière, il a soumis 19 rapports d'audit, et 23 ont été débattus par le Parlement.

\section{3) Procédures parlementaires utilisées dans le traitement des rapports de l'ISC}

Le traitement des rapports du Tribunal de Cuentas est régi par des procédures établies par deux règles de la Commission mixte. Il est également intéressant de consulter les ordonnances du Congrès.

La Commission mixte est généralement présidée par un député du parti majoritaire, dont le choix résulte normalement de négociations politiques.

La Commission ne nomme pas de rapporteur. Les entités contrôlées ou les autres personnes concernées ne sont pas conviées aux auditions afin d'apporter des clarifications ou un complément d'informations. La Commission ne fait pas examiner les rapports par des experts externes indépendants; elle ne demande pas par écrit aux personnes appropriées un complément d'informations ou des clarifications.

Le débat sur les rapports du Tribunal de Cuentas se déroule au sein de la Commission mixte. De manière générale, les autres commissions n'examinent pas les rapports de I'ISC.

À la fin des délibérations sur un rapport d'audit, une résolution accompagnée du rapport est publiée dans le Journal officiel.

\section{Personnel}

La Commission mixte est assistée d'un collaborateur ayant une formation de juriste. Aucun document de recherche écrit, d'analyse ou de note d'informations n'est établi dans le but de préparer les débats sur les rapports du Tribunal de Cuentas.

\section{Participation de I'ISC à l'examen de ses rapports par le Parlement}

Le Président du Tribunal de Cuentas présente périodiquement au Parlement les rapports approuvés et publiés par la Cour tout au long de l'année, car ces rapports sont approuvés en plénière, lors des réunions régulières de la Cour.

Le Tribunal de Cuentas ne participe pas à la préparation des auditions aux côtés de la commission ou du Parlement. Son président, le Chef du cabinet de la présidence et son directeur de la communication assistent aux auditions qui les concernent. 


\section{4) Rapports de l'ISC}

Dans sa communication avec le Parlement, le Tribunal de Cuentas se concentre sur des problèmes génériques / systémiques et sur la formulation de recommandations au Parlement, en tenant compte du périmètre et des perspectives de contrôle déterminés dans le programme de contrôle annuel et les directives techniques de contrôle approuvés pour chaque rapport. Le système de contrôle interne peut être un sujet mis en avant dans les rapports de l'ISC.

Pour terminer, les rapports d'audit ont une structure commune, avec des conclusions et des opinions claires.

\section{5) Suivi des rapports de l'ISC}

Le suivi des conclusions et des recommandations contenues dans les rapports d'audit peut être intégré aux programmes de contrôle annuels. Le Tribunal de Cuentas conserve une trace des conclusions et des recommandations acceptées par les entités contrôlées. Le Parlement suit la mise en œuvre des recommandations formulées dans les rapports d'audit.

Le Tribunal de Cuentas consacre un rapport spécifique à la mise en œuvre / l'ignorance des recommandations, y compris celles relatives à la configuration et au fonctionnement des systèmes de contrôle internes; cette démarche encourage le suivi parlementaire.

Le gouvernement est tenu d'établir un rapport sur la mise en œuvre des recommandations du Tribunal de Cuentas.

Les rapports du Tribunal de Cuentas ne sont pas régulièrement utilisés lors du débat annuel sur les projets de budget.

\section{6) Audits réalisés à la demande du Parlement}

En vertu de la section 45 de la Loi organique, les procédures d'exécution du contrôle sont initiées ex officio à chaque étape par le Tribunal de Cuentas, le Parlement et, au sein de leurs domaines de compétence, par les assemblées législatives régionales.

\section{7) Programme de travail}

Le Tribunal de Cuentas ne consulte pas le Parlement sur son programme de travail en matière de contrôle.

\section{8) Niveau et fréquence des échanges}

Les auditions donnent généralement lieu à des échanges officiels. Les comités directeurs de la Cour et la Commission mixte peuvent avoir des échanges occasionnels.

Il y a des échanges informels entre le Chef du cabinet de la présidence de la Cour et le juriste de la Commission mixte. Le Chef de cabinet est spécialement affecté aux relations parlementaires.

\section{9) Bonnes pratiques}

Les rapports d'audit sont publiés sur le site Internet du Tribunal de Cuentas. Des communiqués de presse portant sur les rapports sont diffusés par le Tribunal de Cuentas. 


\section{0) Perspective}

La présentation des rapports connaît parfois des retards, en raison de l'agenda parlementaire fixé par la Commission mixte, et des modalités de suivi par le Parlement de ses propres résolutions. 


\section{SUÈDE}

Bureau national d'audit

\section{1) Cadre juridique}

Le périmètre et le sujet du contrôle sont régis par la Loi relative au contrôle des activités de l'État et par la Loi contenant des instructions destinées au Bureau national d'audit suédois.

La Constitution, (chapitre 13) :

"Le Bureau national d'audit est une autorité sous la direction du Riksdag (Parlement) dont le rôle est de contrôler les activités de l'État. Les dispositions prévoyant que le contrôle du Bureau national d'audit peut s'étendre également aux activités autres que celle de l'État sont fixées par la loi. »

"Le Bureau national d'audit est sous la direction de trois Auditeurs généraux élus par le Riksdag. Le Riksdag est habilité à révoquer un Auditeur général uniquement si l'Auditeur général ne remplit plus les conditions définies pour cette charge ou a été inculpé pour négligence grave. Les contrôleurs généraux décident indépendamment, dans le respect des règles fixées par la loi, des activités à contrôler. Ils déterminent séparément et individuellement la façon dont ils vont effectuer l'audit et ils formulent leurs propres conclusions en s'appuyant sur leur audit. »

\section{2) Modalités de soumission des rapports de l'ISC au parlement}

Les audits financiers font l'objet de rapports d'audit sur les états financiers, que le Bureau national d'audit suédois (SNAO) soumet au gouvernement et à l'entité contrôlée. Le SNAO fait en outre un rapport oral aux entités contrôlées ainsi qu'aux ministères concernés.

Les audits de performance font l'objet de rapports soumis au Riksdag, qui les transmet à son tour au gouvernement. Le gouvernement doit rendre compte sous quatre mois des mesures qu'il a prises ou qu'il a l'intention de prendre. La commission compétente examine alors le document, et le Riksdag rend sa décision sur la suite de la procédure.

Le SNAO soumet son rapport annuel au Riksdag, accompagné de son rapport financier. Le rapport annuel contient une évaluation des activités et des objectifs du SNAO. Les observations les plus significatives sont intégrées au rapport annuel des Auditeurs généraux.

Dans le rapport de suivi, le SNAO assure un suivi annuel des actions prises suite aux constatations et aux recommandations formulées dans les rapports d'audit.

\section{Procédures d'envoi des rapports au Parlement}

Pour chaque législature, le Parlement nomme un comité parlementaire pour le SNAO. Ce comité comprend un parlementaire par parti. Son rôle est de veiller à ce que les activités d'audit nationales se déroulent en toute transparence. II suit les activités du SNAO, qui lui présente les actions et les résultats des audits.

Le SNAO présente en outre les rapports d'audit de performance aux commissions concernées, à la demande de ces dernières. 
Nombre et type de rapports

\begin{tabular}{|c|c|c|c|c|c|c|c|}
\hline & $\begin{array}{l}\text { Rapports } \\
\text { d'audit } \\
\text { financier }\end{array}$ & \begin{tabular}{|c|} 
Rapports d'audit \\
de conformité
\end{tabular} & $\begin{array}{l}\text { Rapports d'audit } \\
\text { de performance }\end{array}$ & $\mid \begin{array}{c}\text { Autres rapports } \\
\text { ou documents }\end{array}$ & $\begin{array}{l}\text { Rapports } \\
\text { d'activité } \\
\text { annuels }\end{array}$ & $\begin{array}{c}\text { Rapports } \\
\text { demandés par } \\
\text { le Parlement }\end{array}$ & Remarques \\
\hline $\begin{array}{c}\text { Nombre de } \\
\text { rapports soumis }\end{array}$ & 3 & Sans objet & 25 & 3 & 1 & Sans objet & \begin{tabular}{|c|} 
Les rapports \\
d'audit \\
financiers sont \\
principalement \\
remis au \\
gouvernement
\end{tabular} \\
\hline $\begin{array}{l}\text { Nombre de } \\
\text { rapports discutés } \\
\text { par le Parlement }\end{array}$ & 3 & Sans objet & 25 & & 1 & Sans objet & \\
\hline $\begin{array}{l}\text { Soumis au } \\
\text { Président }\end{array}$ & $\mathrm{N}$ & Sans objet & $\mathrm{O}$ & $\mathrm{N}$ & $\mathrm{O}$ & Sans objet & $\begin{array}{c}\text { Au Président, } \\
\text { puis à la } \\
\text { commission } \\
\text { compétente }\end{array}$ \\
\hline $\begin{array}{l}\text { Soumis au } \\
\text { Président de la } \\
\text { commission } \\
\text { spécialisée }\end{array}$ & 3 & Sans objet & 25 & 0 & 0 & Sans objet & Voir ci-dessus \\
\hline $\begin{array}{c}\text { Copies envoyées } \\
\text { aux autres } \\
\text { commissions } \\
\text { concernées }\end{array}$ & $\mathrm{N}$ & Sans objet & 0 & $\mathrm{~N}$ & 0 & Sans objet & \\
\hline $\begin{array}{c}\text { Notes de synthèse } \\
\text { destinées à la } \\
\text { commission } \\
\text { parlementaire }\end{array}$ & 0 & Sans objet & 0 & 0 & 0 & Sans objet & \begin{tabular}{|c|} 
Sur demande. \\
La commission \\
fait appel au \\
SNAO \\
lorsqu'elle le \\
juge \\
nécessaire.
\end{tabular} \\
\hline $\begin{array}{c}\text { Résumés soumis au } \\
\text { Parlement }\end{array}$ & $\mathrm{N}$ & Sans objet & 0 & $\bar{N}$ & $\bar{N}$ & Sans objet & \\
\hline
\end{tabular}

3) Procédures parlementaires utilisées dans le traitement des rapports de l'ISC

Les procédures sont dans une large mesure réglementées par la loi. Toutefois, les commissions permanentes disposent, dans les limites permises par la loi, de diverses modalités de traitement des rapports. 
Les rapports sont étudiés par les commissions permanentes compétentes, en fonction du sujet et du domaine politique évoqués dans le rapport.

Des auditions en présence des entités contrôlées peuvent avoir lieu. II arrive parfois que le SNAO y participe.

Les débats peuvent se dérouler au sein de la commission permanente ou en plénière.

La commission responsable de l'examen du rapport peut inviter d'autres commissions permanentes à commenter le rapport.

Le Parlement envoie le rapport au gouvernement. Ce dernier répond et le rapport du SNAO est annexé à la communication écrite, qui est ensuite débattue par la commission permanente. La commission établit un rapport contenant ses propositions sur la décision de la Chambre au regard de la question étudiée. Ces propositions sont ensuite débattues en séance plénière, puis une décision est prise.

\section{Participation de I'ISC à l'examen de ses rapports par le Parlement}

Le SNAO ne participe pas à la préparation des auditions.

L'Auditeur général est généralement invité à s'exprimer devant la commission. Il est accompagné du chef de projet, mais cela ne signifie pas que le SNAO participe au débat / à la préparation du rapport.

\section{4) Rapports de I'ISC}

Le SNAO intègre dans ses rapports toutes les constatations découlant de l'audit. Les systèmes de contrôle internes peuvent représenter un volet du rapport.

Tous les rapports contiennent des conclusions et la majorité des rapports contiennent des recommandations.

\section{5) Suivi des rapports de I'ISC}

En ce qui concerne les audits financiers, le suivi des constations est assuré dans l'audit de l'année suivante.

En ce qui concerne les audits de performance, le SNAO n'est pas expressément responsable d'assurer le suivi des actions prises. Le gouvernement en revanche est tenu de rendre compte des mesures prises devant le Parlement.

Le SNAO soumet un rapport de suivi annuel qui couvre les mesures prises dans le cadre de plusieurs rapports d'audit.

\section{6) Audits réalisés à la demande du Parlement}

Le SNAO ne reçoit pas de demande du Parlement.

Le SNAO a différents échanges avec le Parlement, les commissions permanentes et le comité parlementaire dédié à I'ISC. Lors de ces échanges, le SNAO aborde naturellement des sujets intéressants en matière d'audit. Toutefois, c'est toujours l'Auditeur général qui choisit les audits à exécuter. 


\section{7) Programme de travail}

Voir ci-dessus. Le SNAO est tenu d'informer le comité parlementaire de son plan de contrôle annuel avant de l'adopter.

\section{8) Niveau et fréquence des échanges}

Des échanges ont lieu à tous les niveaux organisationnels.

Un collaborateur est chargé de coordonner les échanges du SNAO avec le Parlement.

\section{9) Bonnes pratiques}

Après chaque élection générale (tous les quatre ans), le SNAO invite toutes les commissions permanentes à une présentation du SNAO. Cette action aide le Parlement à mieux connaître le rôle du SNAO et elle facilite de surcroît l'accès personnel au SNAO.

Le personnel du SNAO est parfois détaché auprès des commissions permanentes afin d'améliorer la compréhension mutuelle.

10) Y-a-t-il d'autres éléments concernant les relations actuelles entre l'ISC et le Parlement que vous souhaiteriez souligner, et quelles a été leur évolution au cours des cinq dernières années?

Travailler et échanger avec le Parlement développe les connaissances et la compréhension de nos domaines d'activités. À titre d'exemple, inviter les parlementaires après chaque élection constitue une initiative très appréciée. 


\section{TURQUIE}

Cour des comptes

\section{1) Cadre juridique}

\section{Audits et établissement des rapports}

Conformément à la Constitution, la Cour des comptes turque (TCA) contrôle les activités financières, les décisions et les opérations des administrations publiques dans le cadre de leur obligation de rendre compte, puis elle soumet des informations et des rapports, exacts, suffisants et en temps voulu, à la Grande Assemblée nationale turque (TGNA) sur les résultats de ces audits.

La TCA exécute les activités de contrôle pour le compte de la TGNA. L'audit a pour but de soumettre des informations fiables et suffisantes à la TGNA et aux citoyens sur les résultats des administrations publiques, conformément à l'exercice du pouvoir budgétaire. Dans ce contexte, la TCA doit soumettre ses rapports à la TGNA, à l'exception des rapports concernant les administrations locales.

\section{Communication relative au plan de travail de I'ISC}

La TCA élabore ses plans stratégiques annuels et ses programmes de contrôle annuel, en accord avec l'analyse des risques et les attentes de la TGNA, des citoyens et des administrations publiques contrôlées.

\section{Demandes d'audit par la TGNA}

Sur décision des commissions d'étude, d'enquête et de spécialité de la TGNA, si l'audit est limité au sujet demandé, la Présidence de la TGNA a la faculté de demander au TCA de contrôler les comptes et les opérations de toutes les institutions et les organisations publiques, qu'elles soient ou non soumises à un audit. Dans les domaines concernés, il y a les privatisations, les plans incitatifs, la mise en œuvre de prêts et de crédits. Cette règle s'applique également aux comptes et aux opérations des institutions, organisations, fonds, entreprises, sociétés, coopératives, syndicats, fondations, associations, ou de toute entreprise de même ordre qui utilisent des ressources publiques. Les résultats des audits doivent être soumis à la Présidence de la TGNA. Les demandes d'audit provenant de la TGNA sont prioritaires.

\section{2) Modalités de soumission des rapports de l'ISC au parlement}

La TCA envoie essentiellement deux types de rapports à la TGNA :

\section{A) Les rapports généraux}

1) La déclaration de conformité générale est le rapport dans lequel sont évalués la fiabilité et l'exactitude des tableaux et des documents financiers intégrés aux comptes finaux préliminaires du ministère des Finances. Il compare les résultats de l'application de la loi générale relative au budget du gouvernement aux résultats présentés par les comptes des entités publiques. Ce rapport est remis à la Présidence de la TGNA pour être débattu par la Commission de la planification et du budget. Le délai de soumission de ce rapport est fixé au 13 septembre.

2) Le rapport de vérification générale réalisé par un audit externe est le rapport concernant les affaires jugées importantes ou de portée générale figurant dans les rapports d'audit établis à la fin des contrôles des entités publiques. Il comprend en outre des informations générales sur les audits réalisés, ainsi que d'autres problématiques jugées adéquates au contexte financier. Ce rapport est 
remis à la Présidence de la TGNA pour être débattu par la Commission de la planification et du budget. Le délai de soumission de ce rapport est fixé au 13 septembre.

3) Le rapport de vérification général des responsabilités est établi par la TCA lorsqu'elle évalue les rapports de responsabilités envoyés par les administrations publiques, le rapport général des responsabilités du gouvernement local établi par le ministère de l'Intérieur et le rapport général des responsabilités élaboré par le ministère des Finances en examinant les résultats des audits. Le rapport de vérification général des responsabilités est préparé par la TCA et remis à la Présidence de la TGNA pour être débattu par la Commission de la planification et du budget. Le délai de soumission de ce rapport est fixé au 13 septembre.

4) Le rapport de vérification des statistiques financières reprend les résultats de la vérification des statistiques financières publiées par le ministère des Finances pour une année donnée, du point de vue de leur préparation, de leur publication, de leur exactitude, de leur fiabilité et de leur conformité à des normes prédéfinies.

Ce rapport remis à la Présidence de la TGNA pour être débattu par la Commission de la planification et du budget. Le délai de soumission de ce rapport est fixé au 13 septembre.

5) Le rapport général sur les entreprises commerciales d'État regroupe les informations relatives à toutes les entreprises publiques contrôlées. Ces informations portent sur les résultats d'activité annuels, leur place dans l'économie du pays, la situation de l'emploi, leur situation financière, leurs activités commerciales, leurs investissements et leurs filiales, ainsi que les problèmes généraux et communs propres à leur secteur respectif. Ce rapport est remis à la Présidence de la TGNA pour être débattu par la Commission chargée des entreprises commerciales d'État.

B) Les rapports d'audit institutionnels présentent les audits institutionnels annuels exécutés par la TCA.

Ils couvrent les audits de régularité (audits financiers et audits de conformité) et, dans certains cas, les audits de performance (audit des indicateurs de performance). Ces rapports individuels, à l'exception des rapports sur les administrations locales, sont remis à la Présidence de la TGNA pour être débattus par la Commission de la planification et du budget. Ils sont examinés par la TGNA au cours de la négociation budgétaire sur les institutions publiques.

\section{Procédures d'envoi des rapports au Parlement}

Le Président s'entretient une fois par an avec le Président du Parlement pour lui remettre les rapports de la TCA et pour le tenir informé de ses activités. Tous les rapports de la TCA sont publiés sur son site Internet. 
Nombre et type de rapports

\begin{tabular}{|c|c|c|c|c|c|c|c|}
\hline & $\begin{array}{l}\text { Rapports } \\
\text { d'audit } \\
\text { financier }\end{array}$ & \begin{tabular}{|c|} 
Rapports d'audit \\
de conformité
\end{tabular} & $\begin{array}{l}\text { Rapports d'audit } \\
\text { de performance }\end{array}$ & $\begin{array}{c}\text { Autres rapports } \\
\text { ou documents }\end{array}$ & $\begin{array}{l}\text { Rapports } \\
\text { d'activité } \\
\text { annuels }\end{array}$ & $\begin{array}{c}\text { Rapports } \\
\text { demandés par } \\
\text { le Parlement }\end{array}$ & Remarques \\
\hline $\begin{array}{c}\text { Nombre de } \\
\text { rapports soumis }\end{array}$ & & $545^{*}$ & & $74^{* *}$ & $5 * * *$ & & \\
\hline $\begin{array}{c}\text { Nombre de } \\
\text { rapports discutés } \\
\text { par le Parlement }\end{array}$ & & 211 & & 74 & 5 & & \\
\hline $\begin{array}{c}\text { Soumis au } \\
\text { Président }\end{array}$ & & 211 & & 74 & 5 & & \\
\hline $\begin{array}{l}\text { Soumis au } \\
\text { Président de la } \\
\text { commission } \\
\text { spécialisée }\end{array}$ & & 211 & & 74 & 5 & & \\
\hline $\begin{array}{c}\text { Copies envoyées } \\
\text { aux autres } \\
\text { commissions } \\
\text { concernées }\end{array}$ & & & & & & & \\
\hline $\begin{array}{l}\text { Notes de synthèse } \\
\text { destinées à la } \\
\text { commission } \\
\text { parlementaire }\end{array}$ & & & & & & & \\
\hline $\begin{array}{c}\text { Résumés soumis au } \\
\text { Parlement }\end{array}$ & & & & & & & \\
\hline
\end{tabular}

* Les rapports d'audit de régularité réalisés par la TCA présentent les contrôles des indicateurs de performance.

** Rapports sur les entreprises d'État.

*** Rapport de vérification générale par un audit externe, rapport de vérification générale des responsabilités, rapport de vérification des statistiques publiques, déclaration générale de conformité, rapport général sur les entreprises commerciales de l'État.

\section{3) Procédures parlementaires utilisées dans le traitement des rapports de l'ISC}

Les principes et les procédures qui gouvernent les délibérations sur les rapports de la TCA devant la TGNA sont expliquées dans le Règlement intérieur de la TGNA. En vertu de l'Article 20 du Règlement intérieur de la TGNA, la Commission de la planification et du budget examine et vérifie les rapports envoyés par la TCA. Aucune procédure de vérification n'est prescrite par le Règlement intérieur ou par aucun autre document.

Deux commissions de la TGNA sont chargées de discuter des rapports soumis par la TCA. La Commission de la planification et du budget examine les rapports d'audit de régularité (audits financiers et audits de conformité) de toutes les institutions publiques contrôlées par la TCA, à l'exception des administrations locales. Elle est présidée par un membre du parti majoritaire. La Commission chargée des entreprises commerciales de l'État examine les rapports d'audit concernant les entreprises commerciales publiques. 
Elle est présidée par un membre du parti majoritaire. Ces commissions sont fondées sur le principe de négociation.

Au sein de la Commission chargée des entreprises commerciales de l'État, il y a une sous-commission qui comporte un effectif d'assistants et d'experts adéquat. Elle est principalement responsable de l'agenda de la Commission chargée des entreprises commerciales de l'État et des rapports sur les séances de cette Commission. Cette sous-commission joue le rôle de rapporteur pour la Commission chargée des entreprises commerciales de l'État.

Les rapports d'audit institutionnels sont examinés par la Commission de la planification et du budget au cours des négociations concernant le budget des institutions publiques. En conséquence, les membres de la haute direction de ces institutions sont présents aux réunions de la Commission.

Lorsque les rapports sur les entreprises commerciales d'État sont discutés, le président et les membres du conseil de ces entreprises participent à la séance.

La Commission chargée des entreprises commerciales de l'État est habilitée à solliciter des informations ou des clarifications écrites supplémentaires aux personnes concernées.

Le débat sur les rapports de la TCA se déroule en réunion de la commission. Mises à part les commissions compétentes, aucune autre commission n'est habilitée à examiner les rapports de la TCA.

Les rapports des commissions, y compris les minutes des séances, sont rédigées et publiés sur les sites Internet de la TGNA.

\section{Personnel}

La Division du budget est chargée de seconder la Commission de la planification et du budget dans le traitement des rapports de la TCA. Toutefois, cette division a d'autres obligations, comme par exemple le premier examen de l'avant-projet de loi. Treize experts et sept agents administratifs travaillent pour cette division.

La Commission de la planification et du budget peut préparer des questions qu'elle posera en audition.

La sous-commission susmentionnée peut remettre à la Commission chargée des entreprises commerciales de l'État des études écrites, des analyses ou des notes d'information qui seront utilisés lors des débats sur les rapports de la TCA ; elle peut par ailleurs préparer des questions que la commission posera en audition, et rédiger des rapports ou d'autres documents destinés à la commission.

\section{Participation de I'ISC à l'examen de ses rapports par le Parlement}

Lors de la vérification du budget de la TCA, son Président assiste à l'audition conduite par la Commission de la planification et du budget pour présenter à ses membres l'activité générale et les rapports de la TCA. Lorsque les rapports de la TCA constituent l'objet des délibérations, les présidents des chambres, le directeur du service administratif de la TCA, et un nombre suffisant de contrôleurs responsables du rapport sont présents pour fournir des informations, si besoin est. De manière générale, si un membre de la Commission demande des explications sur le rapport, le représentant de la TCA répond à cette question. 
Lorsque les rapports de la TCA sont débattus au sein de la Commission chargée des entreprises commerciales de l'État, le directeur du service administratif de la TCA, le chef du groupe de contrôleurs responsable de l'audit et un nombre suffisant de contrôleurs sont présents pour fournir des informations si besoin est.

\section{4) Rapports de I'ISC}

Dans sa communication avec le Parlement, la TCA se concentre sur des problèmes génériques / systémiques et sur la formulation de recommandations.

La configuration et le fonctionnement des systèmes de contrôle internes sont des sujets mis en avant dans les rapports de la TCA.

Les rapports d'audit financiers contiennent des opinions d'audit. La TCA s'efforce d'exprimer dans ses rapports d'audit des conclusions claires.

\section{5) Suivi des rapports de l'ISC}

En ce qui concerne les rapports sur les institutions publiques de la Commission de la planification et du budget, dans le contexte de la loi générale régissant le budget du gouvernement, les équipes d'audit conservent une trace des conclusions et des recommandations acceptées par l'entité contrôlée, et l'avancement des travaux est consigné dans le rapport d'audit de l'année concernée. Le Parlement n'a pas de mécanisme visant la mise en œuvre des recommandations de la TCA dans ces types de rapports d'audit.

En ce qui concerne les rapports sur les entreprises commerciales d'État de la Commission chargée des entreprises commerciales de l'État, les équipes d'audit compétentes conservent une trace des conclusions et des recommandations acceptées par l'entité contrôlée. Le suivi fait l'objet d'une section spéciale dans le rapport d'audit. Si elle le juge indispensable, la Commission chargée des entreprises commerciales de l'État assure le suivi spécifique des rapports.

\section{6) Audits réalisés à la demande du Parlement}

Sur décision des commissions d'étude, d'enquête et de spécialisation de la TGNA, si l'audit est limité au sujet demandé, la Présidence de la TGNA a la faculté de demander au TCA de contrôler les comptes et les opérations de toutes les institutions et les organisations publiques, qu'elles soient ou non soumises à un audit. Dans les domaines concernés, il y a les privatisations, les plans incitatifs, la mise en œuvre de prêts et de crédits. Cette règle s'applique également aux comptes et aux opérations des institutions, organisations, fonds, entreprises, sociétés, coopératives, syndicats, fondations, associations, ou de toute entreprise du même ordre qui utilisent des ressources publiques. Les résultats des audits doivent être soumis à la Présidence de la TGNA. Les demandes d'audit provenant de la TGNA sont prioritaires.

Un examen préliminaire des demandes d'audit issues des commissions d'étude, d'enquête et de spécialisation de la TGNA est réalisé par le groupe d'assistance nommé par la Présidence. Suite à cet examen préliminaire, le groupe d'assistance soumet son évaluation et son avis à la Présidence dans les dix jours ouvrés. L'examen préliminaire détermine dans quelle mesure les comptes et les opérations sont liés à la demande d'audit, et comment cette dernière aborde les principes et les procédures de contrôle, les délais et les ressources budgétaires, qui sont présentés clairement dans la Loi relative à l'ISC et dans d'autres lois. 


\section{7) Programme de travail}

La TCA élabore son programme de travail en toute indépendance par rapport aux autres organes, y compris à la TGNA et aux entités contrôlées. Elle est habilitée à choisir les entités et les domaines à contrôler. C'est une condition indispensable à son indépendance, de sorte qu'elle ne consulte aucune organisation dans le cadre de son programme de travail. Les demandes d'audit de la TGNA sont toutefois prioritaires dans le programme de travail.

\section{8) Niveau et fréquence des échanges}

La Commission de la planification et du budget a des contacts lors de la préparation du budget. Le groupe de la TCA chargé de la conformité générale est présent pendant les négociations budgétaires au sein de la Commission et en séance plénière de l'Assemblée générale. Le Président de la TCA informe la Commission de la planification et du budget au moins deux fois par an et, si nécessaire, les commissions compétentes, sur les activités de la Cour des comptes. Une équipe d'audit de la TCA est toujours présente à la TGNA pour travailler sur les activités de contrôle de l'Assemblée.

La Commission chargée des entreprises commerciales de l'État a des échanges réguliers, selon des procédures efficientes au niveau des groupes de travail.

\section{9) Bonnes pratiques}

La TCA a mis sur pied une stratégie de communication destinée à la TGNA, pour faire progresser leurs relations. Dans ce document, la TCA expose les activités nécessaires à la consolidation des relations entre les deux institutions.

Dans le cadre des rapports sur les entreprises commerciales d'État, la commission compétente prête une attention toute particulière au suivi des constations et des recommandations formulées dans les audits.

\section{0) Perspective}

Puisqu'un nombre considérable de rapports d'audit est soumis à la TGNA, cette dernière a quelques difficultés à examiner ces rapports. Si un sous-groupe de travail discutait les rapports de manière détaillée puis les soumettait avec leurs commentaires à la Commission de planification et du budget, la Commission serait en mesure de conduire un examen plus efficient et de prendre les mesures nécessaires. 


\section{ROYAUME-UNI}

Bureau national d'audit

\section{1) Cadre juridique}

Le Bureau national d'audit (NAO) aide le Parlement à rendre le gouvernement responsable de la manière dont il dépense l'argent public et à améliorer la fourniture des services publics. II a pour principal rôle d'examiner les dépenses publiques pour le compte du Parlement.

Le NAO est une institution indépendante du gouvernement. Le directeur du NAO est le Contrôleur général des comptes (C\&AG), qui est un fonctionnaire de la Chambre des Communes nommé par la Couronne.

Le Cadre juridique du NAO est défini par plusieurs lois, en vertu desquelles :

- Il contrôle les états financiers de tous les ministères du gouvernement central, des agences et des autres établissements publics, puis il rend son rapport au Parlement.

- Il établit des rapports coût - résultat (de la performance) qui étudient l'efficacité, l'efficience et l'économie des dépenses du gouvernement.

La loi accorde au C\&AG une entière discrétion dans l'exercice de ses fonctions et, en particulier, lorsqu'il doit décider s'il convient d'exécuter des examens coût - résultat. Lorsqu'il décide de réaliser ces examens, le C\&AG doit " prendre en compte toute proposition faite par la Commission des comptes publics ", qui est la commission parlementaire chargée de la plupart des travaux du NAO.

Le C\&AG est également tenu par la loi d'estimer annuellement l'utilisation des ressources par le NAO, estimation qui sera soumise à l'approbation du Parlement.

\section{2) Modalités de soumission des rapports de l'ISC au parlement}

Les rapports peuvent être soumis uniquement au Parlement lorsqu'il siège, sous réserve des conditions prioritaires suivantes :

- Les résultats des audits financiers réalisés par le NAO sont communiqués séparément au Parlement sous la forme d'une opinion publiée avec les états financiers annuels par organe et, si le C\&AG le juge nécessaire, dans un rapport joint. Les états des audits financiers doivent être publiés avant le 31 janvier suivant la fin de l'exercice financier (qui se termine le 31 mars). Dans la pratique, la majorité des états financiers sont publiés dans les quatre mois qui suivent la fin de l'exercice financier. Le NAO n'établit pas de rapports de conformité individuels car la conformité est traitée dans ses audits financiers.

- Le NAO communique séparément au Parlement les résultats de ses audits coût - résultat, à sa discrétion. II les adresse à la Chambre des Communes dans son ensemble, et non au Président ou à d'autres membres ou commissions de la Chambre. Tous ses rapports coût - résultat (et les rapports sur les états financiers) sont publiés. Préalablement à leur publication, le NAO envoie par courriel des copies sous embargo de tous ses rapports (et les communiqués de presse liées) au Président et aux membres de la Commission des comptes publics. Le NAO envoie également des copies sous embargo aux membres de la Chambre des Communes qui ont sollicité une copie 
de tous les rapports (ou de ceux traitant d'un sujet particulier), ainsi qu'aux membres des autres commissions de la Chambre qui s'intéressent à l'objet du rapport.

- Le NAO produit une large gamme de documents écrits, officiels et officieux, à l'intention d'autres commissions parlementaires sur des problématiques spécifiques dans lesquelles le NAO a une expertise. Certains de ces documents sont directement soumis sous la forme de mémorandums à la commission compétente. D'autres, tels que les 17 petits guides produits par le NAO à l'intention des comités restreints pour leur présenter les différents services de manière simple, sont publiés par le NAO sans les déposer officiellement devant le Parlement.

- Le NAO rédige et soumet au Parlement son rapport et ses comptes annuels. II publie en outre un document de stratégie annuel déterminant ses plans pour les trois prochaines années. Des copies de ces deux documents sont envoyées à la Commission des comptes publics (TPAC, organe établi par la loi et composé de membres du Parlement ayant pour mission d'examiner et d'adopter son budget) et au Comité des comptes publics.

Dans un nombre restreint de cas, le NAO, plutôt que l'organisme contrôlé, communique au Parlement les résultats de son audit financier.

\section{Procédures d'envoi des rapports au Parlement}

Les rapports que le NAO dépose officiellement devant le Parlement sont enregistrés par la Chambre des Communes (ils reçoivent un numéro de la Chambre des Communes figurant sur la page de garde du rapport). La procédure demande au NAO de remettre à la Chambre deux copies papier du rapport et une lettre d'accompagnement.

Comme il l'a été souligné plus haut, le NAO envoie par courriel des copies sous embargo de tous ses rapports (et les communiqués de presse liées) au Président et aux membres de la Commission des comptes publics, aux membres de la Chambre des Communes qui ont sollicité une copie de ses rapports, ainsi qu'aux membres des autres commissions de la Chambre qui s'intéressent à l'objet du rapport.

La majorité des rapports coût-résultat sont examinés par la Commission des comptes publics. Les procédures utilisées par le NAO pour informer la Commission sont présentées ci-dessous. Le NAO fournit des notes d'information sur ses rapports aux autres commissions parlementaires ou aux membres individuels du Parlement, à leur demande. 
Nombre et type de rapports

\begin{tabular}{|c|c|c|c|c|c|c|c|}
\hline & $\begin{array}{l}\text { Rapports } \\
\text { d'audit } \\
\text { financier }\end{array}$ & $\begin{array}{l}\text { Rapports d'audit } \\
\text { de conformité }\end{array}$ & $\begin{array}{l}\text { Rapports d'audit } \\
\text { de performance }\end{array}$ & $\begin{array}{c}\text { Autres } \\
\text { rapports ou } \\
\text { documents }\end{array}$ & $\begin{array}{l}\text { Rapports } \\
\text { d'activité } \\
\text { annuels }\end{array}$ & $\begin{array}{c}\text { Rapports } \\
\text { demandés } \\
\text { par le } \\
\text { Parlement }\end{array}$ & Remarques \\
\hline $\begin{array}{c}\text { Nombre de } \\
\text { rapports soumis }\end{array}$ & 368 & Sans objet & 60 & 0 & 2 & 0 & \begin{tabular}{|c|} 
Tous les chiffres \\
correspondent à \\
l'exercice financier \\
2015-16
\end{tabular} \\
\hline $\begin{array}{c}\text { Nombre de } \\
\text { rapports discutés } \\
\text { par le Parlement }\end{array}$ & 0 & Sans objet & 31 & 0 & 2 & 0 & \\
\hline $\begin{array}{l}\text { Soumis au } \\
\text { Président }\end{array}$ & \multicolumn{7}{|c|}{$\begin{array}{l}\text { Les rapports sont adressés à la Chambre des Communes dans son ensemble, et pas à son Président ou à des } \\
\text { membres individuels, ou à des commissions de la Chambre. }\end{array}$} \\
\hline $\begin{array}{l}\text { Soumis au } \\
\text { Président de la } \\
\text { commission } \\
\text { spécialisée }\end{array}$ & \multicolumn{7}{|c|}{ Voir ci-dessus } \\
\hline $\begin{array}{l}\text { Copies envoyées } \\
\text { aux autres } \\
\text { commissions } \\
\text { concernées }\end{array}$ & Non connu & Sans objet & Non connu & 0 & 2 & 0 & $\begin{array}{c}\text { Le NAO ne } \\
\text { conserve pas } \\
\text { d'informations } \\
\text { détaillées à ce } \\
\text { sujet }\end{array}$ \\
\hline $\begin{array}{c}\text { Notes de synthèse } \\
\text { destinées à la } \\
\text { commission } \\
\text { parlementaire }\end{array}$ & Non connu & Sans objet & Au moins 31 & 0 & 0 & 0 & $\begin{array}{c}\text { Le NAO ne } \\
\text { conserve pas } \\
\text { d'informations } \\
\text { détaillées à ce } \\
\text { sujet }\end{array}$ \\
\hline $\begin{array}{c}\text { Résumés soumis } \\
\text { au Parlement }\end{array}$ & \multicolumn{7}{|c|}{ Le NAO ne soumet pas de résumé de ses rapports au Parlement } \\
\hline
\end{tabular}

\section{3) Procédures parlementaires utilisées dans le traitement des rapports de l'ISC}

Pour traiter les rapports du NAO, il n'y pas de procédure établie ou de commission compétente prescrite par les règles de procédure du Parlement (ordonnance). Dans la pratique néanmoins, le Comité des comptes publics (PAC), désigné par la Chambre des Communes pour examiner « les comptes ... déposés devant le Parlement ... » remplit ce rôle.

Comprenant quinze membres, le PAC qui, par convention, est présidé par un membre de l'opposition, est élue par la Chambre des Communes au moyen d'ordonnances. Le Comité est habilité à convoquer des personnes, des documents et des dossiers et à nommer des conseillers spécialisés, soit pour fournir des informations indisponibles directement, soit pour éclairer des points complexes au sein de l'ordre de référence du Comité. 
Le PAC nomme généralement parmi ses membres un rapporteur lorsqu'il enregistre les témoignages oraux publics du ministère concerné (et d'autres témoins experts) sur chaque rapport coût-résultat qu'il décide d'examiner. Ce rapporteur dirige habituellement les questions posées aux témoins, mais le Président et d'autres membres du PAC posent également des questions. Suite à la séance de témoignages, le Comité publie normalement son propre rapport accompagné de recommandations sur l'objet de l'examen. II publie normalement une cinquantaine de rapports par an. Par convention, le gouvernement est tenu de répondre à ces recommandations dans les deux mois suivant leur publication.

Comme les rapports du NAO sont soumis à la Chambre dans son ensemble (et pas à une commission particulière) et publiés, toute commission peut décider de les examiner. En pratique, les matériaux contenus dans ces rapports sont souvent cités par les commissions pendant leurs enquêtes et en séance plénière de la Chambre, mais il est rare que d'autres commissions tiennent des séances de témoignage spécifiques sur l'un des rapports.

Il n'y a pas de débat dédié aux travaux du NAO ou à ceux du PAC.

Le Parlement possède une procédure de décharge établie. Le NAO rend compte des circonstances qui ont conduit les ministères à commettre une violation budgétaire, et qui l'ont conduit à émettre une opinion avec réserves sur les états financiers. Ces faits sont souvent appelés des votes de dépassement car le ministère d'État a dépassé les ressources, ou une certaine catégorie de ressources, votées par le Parlement. En vertu d'ordonnances, le PAC est sollicité pour examiner les raisons pour lesquelles les organes ont dépassé leurs ressources, et pour demander à la Chambre si elle a une objection à octroyer la somme permettant d'éliminer les dépassements mentionnés dans les rapports. Les éléments contenus dans le rapport du Comité constituent le socle de la déclaration de dépassement établie par le Trésor de sa Majesté, qui est déposée devant le Parlement, qui la régularisera sous la forme d'un projet de loi. La motion visant l'adoption des sommes nécessaires est présentée à la Chambre sans débat, si le Comité a recommandé d'octroyer ces sommes.

\section{Personnel}

Le personnel du PAC comprend six collaborateurs : deux assistants administratifs, trois assistants du comité, et un responsable chargé des médias. Ils fournissent des conseils sur les procédures et une assistance administrative au Comité, entre autres pour la gestion de la correspondance du Comité, l'organisation des séances de témoignage, la publication des rapports du Comité et le traitement des demandes des médias.

Le C\&AG est un témoin permanent aux séances de témoignage du PAC et, avec le personnel du NAO, il remet des notes d'informations sur toute demande de renseignement émise par le Comité, et il aide le Comité à préparer ses propres rapports.

\section{Participation de l'ISC à l'examen de ses rapports par le Parlement}

Préalablement aux séances de témoignage du PAC, le NAO fournit une note d'informations écrite qui suggère des questions à poser. Comme il l'a été souligné plus haut, le C\&AG et le personnel du NAO assistent aux séances de témoignage pour apporter des informations au Comité et pour clarifier les problématiques débattues pendant la séance. Le NAO rédige la première version du rapport du Comité et le lui soumet pour examen. C'est ensuite le tour du Président puis du Comité au complet d'examiner la version préliminaire, en y apportant les modifications qu'ils jugent utiles avant de donner leur accord pour la publication. 


\section{4) Rapports de I'ISC}

\section{Examen des rapports par le Parlement}

Le NAO produit une série de documents très divers. Tandis que les systèmes de contrôle internes n'est pas un sujet mis en avant dans ses rapports, il aborde ces éléments dans ses audits financiers, qui comprennent également des travaux d'assurance plus larges, tels que la vérification des demandes de subventions, l'examen des systèmes informatiques et la certification des économies déclarées par les ministères.

Le programme coût-résultat du NAO comprend des études sur les problèmes génériques / systémiques et sur des rapports qui font des comparaisons transversales au secteur public. Le NAO concentre ses efforts sur les domaines prioritaires du Parlement, et il mène des investigations motivées par des soupçons de fraude, de pratique impropre ou de faiblesse systémique. Le rapport coût-résultat portant sur la réalisation d'un programme ou d'une politique particulière peut traiter des systèmes de contrôle interne, si les sujets et les circonstances s'y prêtent.

La majorité des rapports d'audit du NAO contiennent des conclusions et des opinions claires. Toutefois, les investigations sont plus ciblées que ses autres études coût-résultat, offrant des moyens réactifs et rapides de traiter les préoccupations et les problématiques signalées au NAO. Les investigations produisent des messages ciblés sur l'offre de services publics et elles ne contiennent pas nécessairement des conclusions et des opinions d'audit.

\section{5) Suivi des rapports de I'ISC}

Le gouvernement doit répondre aux recommandations formulées dans les rapports du PAC dans les deux mois suivant leur publication. II publie normalement une Note du Trésor environ tous les mois, correspondant à un ensemble de rapports du PAC. Le NAO vérifie ces réponses et donne des conseils au Comité sur les actions de suivi à mettre en œuvre. Chaque année, le Comité tient des séances de témoignage pour mettre devant leurs responsabilités les organes dont les réponses aux recommandations du Comité, ou l'avancement de leur mise en œuvre, ne paraît pas satisfaisant.

Les entités contrôlées ne sont pas tenues de publier des réponses aux rapports du NAO. Toutefois, le NAO vérifie et discute avec les ministères la mise en œuvre de toutes les recommandations, de manière informelle et en participant officiellement aux comités de contrôle des ministères. Le NAO intègre cet élément dans son prochain programme de travail.

\section{6) Audits réalisés à la demande du Parlement}

Aucune disposition juridique ne permet au Parlement de solliciter des audits. Le C\&AG a, de par la loi, une discrétion complète dans l'exercice de ses fonctions.

Les parlementaires et les commissions demandent au NAO des avis consultatifs ou des examens coûtrésultat sur des sujets spécifiques.

Le NAO s'efforce toujours de proposer une réponse constructive aux questions soulevées et parfois, cette démarche aboutit à des investigations et rapports coût-résultat complets. 


\section{7) Programme de travail}

Le C\&AG doit, conformément à la loi, "prendre en compte toute proposition émanant du Comité des comptes publics » lorsqu'il détermine les examens coût-résultat à conduire. Ainsi, à intervalles réguliers, le NAO discute son prochain programme avec le Comité. Des suggestions d'enquête peuvent toutefois être formulées par le Comité à n'importe quel moment. Toute modification ou nouvelle proposition fait l'objet d'un examen sérieux et est généralement acceptée.

\section{8) Niveau et fréquence des échanges}

En qualité de témoin permanent aux séances de témoignage du PAC, le C\&AG assiste aux réunions du Comité deux fois par semaine, lors des séances du Parlement. Ce contact est complété par des réunions informelles, notamment avec le Président, qui ont lieu périodiquement tout au long de l'année. Le C\&AG rencontre en outre les présidents des autres commissions et d'autres parlementaires pour discuter des affaires pour lesquelles ils ont un intérêt commun.

Dans le cadre du travail, il y a des échanges quotidiens avec le Comité et son personnel.

Le NAO dispose d'une équipe dédiée aux relations parlementaires équivalant à sept postes et demi à temps plein. Le directeur de cette équipe a des échanges quotidiens avec le Président du PAC, de même que le membre de l'équipe qui apporte une assistance directe au Président avant les séances de témoignages, ainsi que des conseils sur les études à venir.

\section{9) Bonnes pratiques}

Voir les réponses aux sections 10 et 11 ci-dessous.

\section{0) Perspective}

Le NAO a pour but d'être une source d'informations et de conseils faisant autorité au sein du Parlement, pour obliger le gouvernement à rendre compte de manière plus efficace. Le NAO est en train d'élargir son influence auprès du Parlement, en améliorant ses communications et en mettant davantage l'accent sur ses relations avec les parlementaires, leurs assistants parlementaires et le personnel parlementaire important.

Le Comité des comptes publics étant le premier client du NAO, ce dernier travaille en étroite collaboration avec lui lorsqu'il élabore son programme de travail. Le NAO est par ailleurs en train d'approfondir la coopération avec le Parlement, intensifiant l'assistance qu'il offre aux deux chambres ainsi qu'aux comités restreints et aux parlementaires en général.

Le NAO a l'intention de coopérer plus étroitement avec le Parlement en développant sur mesure des produits et des services d'assistance répondant aux attentes des parlementaires. Pour cela, le NAO améliore sa connaissance de la Chambre des Communes par des opérations de détachement et de recrutement. Ses nouveaux directeurs des relations parlementaires et des relations externes détiennent une expérience approfondie dans le travail avec le Parlement. Le NAO gère des détachements réciproques avec le personnel des principales commissions et des autres organes de la Chambre, et noue des relations plus étroites avec la bibliothèque de la Chambre.

11) Y-a-t-il d'autres éléments concernant les relations actuelles entre l'ISC et le Parlement que vous souhaiteriez souligner, et quelles a été leur évolution au cours des cinq dernières années? 
Récemment, avec la hausse des ressources destinées à élargir l'assistance apportée au Parlement, le NAO a consolidé ses liens avec d'autres comités restreints, pour les aider de différentes manières, par exemple en proposant des présentations d'information écrites et orales, des détachements de personnel et des détachements à court terme auprès des commissions, et en se présentant devant elles pour apporter des témoignages oraux et officiels. Ces travaux ont été réalisés avec l'appui sans réserve du PAC et de la TPAC, et ils ont été acceptés par les ministères. 


\section{LE PROGRAMME SIGMA}

Le programme SIGMA (Soutien à l'amélioration des institutions publiques et des systèmes de gestion dans les pays d'Europe centrale et orientale) est une initiative conjointe de l'OCDE et de l'Union européenne, financée principalement par I'Union européenne. Depuis 25 ans, SIGMA aide les pays à renforcer leurs systèmes de gouvernance publique et les capacités de leur administration publique.

En partenariat avec la Commission européenne (CE), la Direction générale pour les négociations de voisinage et d'élargissement (DG NEAR), nous travaillons actuellement avec les pays suivants :

- Albanie, Bosnie-Herzégovine, ex-République yougoslave de Macédoine, Kosovo, Monténégro, Serbie et Turquie en tant que pays candidats et candidats potentiels à I'UE

- Algérie, Arménie, Azerbaïdjan, Égypte, Géorgie, Jordanie, Liban, Moldavie, Maroc, Tunisie et Ukraine en tant que pays voisins de l'UE

SIGMA offre une assistance essentiellement dans les 5 domaines suivants :

1. Cadre stratégique de la réforme de l'administration publique

a) Développement et coordination de politiques

b) Gestion des services publics et des ressources humaines

c) Obligation de rendre compte

d) Fourniture de services

e) Gestion des finances publiques, marchés publics et audits externes

SIGMA examine et apporte ses vues sur :

- Les systèmes de gouvernance et les institutions

- Les cadres juridiques

- Les stratégies de réforme et les plans d'action

- L'avancement de la mise en œuvre des réformes

SIGMA offre :

- Des conseils sur la conception et la priorisation des réformes

- Des méthodologies et des outils d'assistance à la mise en œuvre

- Des recommandations sur l'amélioration des lois et les modalités administratives

- Des opportunités de partager les bonnes pratiques, parmi lesquelles des événements régionaux, utilisées par de nombreux pays

- Des documents de stratégie et des études comparatives multi-pays.

Pour avoir des informations complémentaires sur SIGMA, consultez notre site Internet : www.sigmaweb.org

\section{OECD 2017}

Comme SIGMA dépend de I'Organisation de coopération et de développement économiques (OCDE), les mêmes conditions d'utilisation s'appliquent à ses publications : http://www.oecd.org/termsandconditions 